\title{
Employing E-Learning to promote smoking cessation care
}

Citation for published version (APA):

de Ruijter, D. F. L. (2018). Employing E-Learning to promote smoking cessation care: development and evaluation of a tailored e-learning program to support practice nurses to optimally adhere to evidencebased-smoking cessation guidelines. [Doctoral Thesis, Maastricht University]. Datawyse / Universitaire Pers Maastricht. https://doi.org/10.26481/dis.20181107dr

Document status and date:

Published: 01/01/2018

DOI:

10.26481/dis.20181107dr

Document Version:

Publisher's PDF, also known as Version of record

\section{Please check the document version of this publication:}

- A submitted manuscript is the version of the article upon submission and before peer-review. There can be important differences between the submitted version and the official published version of record.

People interested in the research are advised to contact the author for the final version of the publication, or visit the DOI to the publisher's website.

- The final author version and the galley proof are versions of the publication after peer review.

- The final published version features the final layout of the paper including the volume, issue and page numbers.

Link to publication

\footnotetext{
General rights rights.

- You may freely distribute the URL identifying the publication in the public portal. please follow below link for the End User Agreement:

www.umlib.nl/taverne-license

Take down policy

If you believe that this document breaches copyright please contact us at:

repository@maastrichtuniversity.nl

providing details and we will investigate your claim.
}

Copyright and moral rights for the publications made accessible in the public portal are retained by the authors and/or other copyright owners and it is a condition of accessing publications that users recognise and abide by the legal requirements associated with these

- Users may download and print one copy of any publication from the public portal for the purpose of private study or research.

- You may not further distribute the material or use it for any profit-making activity or commercial gain

If the publication is distributed under the terms of Article $25 \mathrm{fa}$ of the Dutch Copyright Act, indicated by the "Taverne" license above, 


\title{
EMPLOYING E-LEARNING TO PROMOTE SMOKING CESSATION CARE
}

\author{
Development and evaluation of a tailored \\ e-learning program to support practice nurses to \\ optimally adhere to evidence-based smoking \\ cessation guidelines
}


ISBN 978-94-6380-025-9

The research presented in this dissertation was conducted at the Care and Public Health Research Institute (CAPHRI), Department of Health Promotion at Maastricht University. The research was funded by the Dutch Cancer Society (KWF Kankerbestrijding). CAPHRI participates in the Netherlands School of Public Health and Care Research CaRe 


\title{
EMPLOYING E-LEARNING TO PROMOTE SMOKING CESSATION CARE Development and evaluation of a tailored e-learning program to support practice nurses to optimally adhere to evidence-based smoking cessation guidelines
}

\author{
Proefschrift \\ ter verkrijging van de graad van doctor aan de Universiteit Maastricht, \\ op gezag van de Rector Magnificus, Prof.dr. Rianne M. Letschert \\ volgens het besluit van het College van Decanen, \\ in het openbaar te verdedigen \\ op woensdag 7 november 2018 om 16.00 uur
}

door

Dennis Franciscus Ludovicus de Ruijter

geboren op 15 februari 1990

Maastricht 


\section{Promotor}

Prof. dr. H. de Vries

\section{Copromotores}

Dr. C. Hoving

Dr. E.S. Smit (Universiteit van Amsterdam)

\section{Beoordelingscommissie}

Prof. dr. S.P.J. Kremers (voorzitter)

Prof. dr. C.A.W. Bolman (Open Universiteit, Heerlen)

Prof. dr. N.H. Chavannes (Leids Universitair Medisch Centrum)

Prof. dr. J.M.W. Muris

Prof. dr. M.C. Willemsen 


\section{Outline}

$\begin{array}{lll}\text { Chapter } 1 \text { General introduction } & 7\end{array}$

Chapter 2 Understanding Dutch practice nurses' adherence to evidence-based smoking cessation guidelines and their needs for web-based adherence support: results from semistructured interviews

Chapter 3 Dutch practice nurses' adherence to evidence-based smoking cessation treatment guidelines

Chapter 4 Web-based computer-tailoring for practice nurses aimed to improve smoking cessation guideline adherence:

A study protocol for a randomized controlled effectiveness trial.

Chapter 5 The effectiveness of a computer-tailored e-learning program for practice nurses to improve their adherence to smoking cessation counseling guidelines: randomized controlled trial

Chapter 6 An economic evaluation of a computer-tailored e-learning program to promote smoking cessation counseling guideline adherence among practice nurses

Chapter 7 General discussion

Valorization Addendum

References

Summary

Samenvatting 169

Dankwoord

Curriculum Vitae

Publication list 

Chapter

General introduction 


\section{Smoking and smoking cessation}

\section{The smoking epidemic}

Smoking is the most preventable cause of non-communicable diseases, such as cancer and cardiovascular disease, and premature death. Worldwide $20.7 \%$ of adults, or 1.1 billion people (i.e. 950 million men and 177 million women), still smoked in 2015. As a consequence, over 7 million lives are lost each year because of smoking-related consequences, contributing to an estimated yearly economic burden of 1.4 trillion dollars related to healthcare costs and loss in productivity (WHO, 2017). In the Netherlands, smoking prevalence is above the global average, as $24.1 \%$ of adults smoked in 2016, of which $77 \%$ reported to smoke on a daily basis (Springvloet \& van Laar, 2017). Nearly 20,000 Dutch adults die of smoking-related causes each year, contributing to $13.1 \%$ of the total burden of disease in the Netherlands (van Laar et al., 2016). These figures illustrate that the smoking epidemic is still far from resolved and continues to have a negative impact on global and national public health.

\section{Smoking cessation measures in the Netherlands}

To reduce the negative impact of smoking on public health, continued efforts are needed to prevent people from smoking tobacco and to support current smokers to successfully quit smoking. Therefore, effective smoking cessation strategies and interventions are essential. National public health policies such as raising tobacco taxes, implementing a smoking ban in public places and an advertising ban at points of sale are effective strategies to discourage people to smoke and prevent current non-smokers from taking up smoking in the future (WHO, 2017). Also in the Netherlands such anti-smoking policies (e.g. regular tax increases on tobacco products since 1997, a national smoking restriction in workplaces since 2002) have been implemented during the past decades (Heijndijk \& Willemsen, 2015). As the Dutch population increasingly supports national smoking bans, the implementation of additional public smoking restrictions can further contribute to the 'de-normalization of smoking' in the Netherlands (Hummel, Willemsen, de Vries, Monshouwer, \& Nagelhout, 2017). Despite these promising developments on political level, it is not sufficient to only rely on governmental, top-down approaches to fight the Dutch smoking epidemic. Especially when targeting current smokers, who might be highly addicted and not motivated to quit, additional quit smoking measures are warranted (Warner \& Burns, 2003). In the Netherlands, 73\% of adult smokers has ever tried to quit smoking and $25 \%$ intends to quit smoking within six months (van Laar et al., 2016), but without a form of smoking cessation support their chance of successfully quitting is only 5\% (Van den Berg \& Schoemaker, 2010).

Therefore, it is important to also consider individual, bottom-up strategies to support current smokers to quit smoking. During the past decades several strategies have globally proven to be effective to support individual smokers to quit, ranging from various types 
of web-based smoking cessation interventions (Taylor et al., 2017) to smoking cessation counseling by a healthcare professional (Rice, Heath, Livingstone-Banks, \& HartmannBoyce, 2017; Stead et al., 2013) or a combination of counseling and pharmacotherapy (Stead, Koilpillai, Fanshawe, \& Lancaster, 2016). Also in the Netherlands such smoking cessation interventions have proven to be effective to support smokers to quit. For example, several tailored web-based interventions demonstrated to be effective to promote individual smoking cessation, either in a text-based (Elfeddali, Bolman, Candel, Wiers, \& de Vries, 2012; Smit, de Vries, \& Hoving, 2012) or video-based format (Stanczyk, Bolman, et al., 2014); counseling interventions consisting of support from healthcare professionals in general practice (Pieterse, Seydel, de Vries, Mudde, \& Kok, 2001) or in a hospital setting (Berndt et al., 2014) significantly improved counseled patients' quit rates; and similar results were found when smoking cessation counseling was combined with pharmacotherapy in general practice (van Rossem et al., 2017). These examples illustrate the various strategies that have been tested in the Netherlands to support current smokers to quit smoking. To ensure that smokers are optimally exposed to individual, bottomup smoking cessation interventions, it is important that as many smokers as possible are reached with these interventions. For this reason, especially individual counseling interventions in general practice have the potential to be a highly accessible form of support for smokers. More specifically, as more than two thirds of Dutch smokers visit their general practitioner (GP) at least once a year (Springvloet \& van Laar, 2017), the majority of smokers can be reached through general practice.

\section{Smoking cessation in general practice care}

In Dutch general practice, smoking cessation support was traditionally predominantly provided by the GP, but this cessation support lacked an evidence-based approach for a long time. It was only until the early 1990s that a structured, evidence-based counseling protocol was developed for GPs, which proved to be effective in improving smoking patients' quit rates (Pieterse et al., 2001). As a result, evidence-based smoking cessation counseling became part of practice guidelines for GPs and subsequently became integrated in routine smoking cessation care in general practice (Trimbos-instituut \& NHG, 2016). Yet, during the past decades a shift has taken place towards more care responsibilities, including smoking cessation counseling, being provided by a practice nurse (PN) instead of the GP, to relieve the workload for GPs (Dierick-van Daele, Metsemakers, Derckx, Spreeuwenberg, \& Vrijhoef, 2009; van Hassel, Batenburg, \& van der Velden, 2016).

PNs are employed in $88 \%$ of Dutch general practices and a single practice employs two part-time PNs for every fulltime GP on average (van Hassel et al., 2016). In this dissertation, PNs are referred to as trained nurses that particularly deal with patients with chronic conditions (i.e. POH), as opposed to PNs treating patients with mental health 
problems in general practice (i.e. POH-GGZ) (Freund et al., 2015). PNs are hence responsible for chronic disease care, such as care for patients with diabetes, cardiovascular diseases and COPD, and lifestyle counseling activities (Dierick-van Daele et al., 2009; van Hassel et al., 2016). As part of these activities, PNs are also responsible for smoking cessation counseling in general practice, often in collaboration with the GP. Moreover, during their post-graduate training, PNs are instructed to apply evidence-based guidelines for treating and counseling their patients (Heiligers et al., 2012), hence including training in providing evidence-based smoking cessation care. The quality of care provided in Dutch general practice was found to be comparable when provided by either PNs or GPs (Dierick-van Daele et al., 2009) and recently smoking cessation support from a PN or GP proved to be equally successful (van Rossem et al., 2017). These results specifically illustrate the important role a PN nowadays plays in delivering smoking cessation care to their patients.

When smoking patients visit their general practice, the Dutch treatment guideline of tobacco addiction and smoking cessation support advocates that each patient should always receive a quit smoking advice from their GP or PN, also when a smoking patient visits for a non-smoking related consultation (Trimbos-instituut \& NHG, 2016). As evidence demonstrated that more intense forms of smoking cessation counseling are more effective in supporting smokers to quit than a quit advice only (Stead et al., 2013), Dutch healthcare professionals are advised to subsequently address smoking cessation more elaborately with their patients (Trimbos-instituut \& NHG, 2016). Therefore, after a GP or a PN has provided a quit advice, smoking patients should enroll in a counseling trajectory. Moreover, patients that actively seek smoking cessation support from their GP or PN, should also enroll in a counseling trajectory (van Laar et al., 2016). As nowadays PNs are mostly responsible for smoking cessation activities in general practice, patients usually enroll in a smoking cessation counseling trajectory with a PN. To structure their counseling, PNs should apply an evidence-based smoking cessation counseling guideline when supporting patients to successfully quit smoking. A recent update of the Dutch treatment guideline described the smoking cessation standard of the Dutch GP association (NHG standard) as the preferred evidence-based counseling guideline for smoking cessation support (Trimbos-instituut \& NHG, 2016), which is to a large extent still based on the evidence-based approach developed in the 1990s (Pieterse et al., 2001).

\section{The Dutch smoking cessation guideline}

In general, the Dutch evidence-based treatment guideline on smoking cessation describes a stepwise approach towards smoking cessation counseling (Figure 1), including steps such as 1) provide a quit advice, 2) assess smoking profile, 3a) assess motivation, 3b) increase motivation, 4a) explore barriers, 4b) discuss/remove barriers, 5) discuss cessation aids, 6) help set a quit date and develop a quit plan, and 7) offer support after the quit date (Trimbos-instituut \& NHG, 2016). 
1. Provide a quit advice

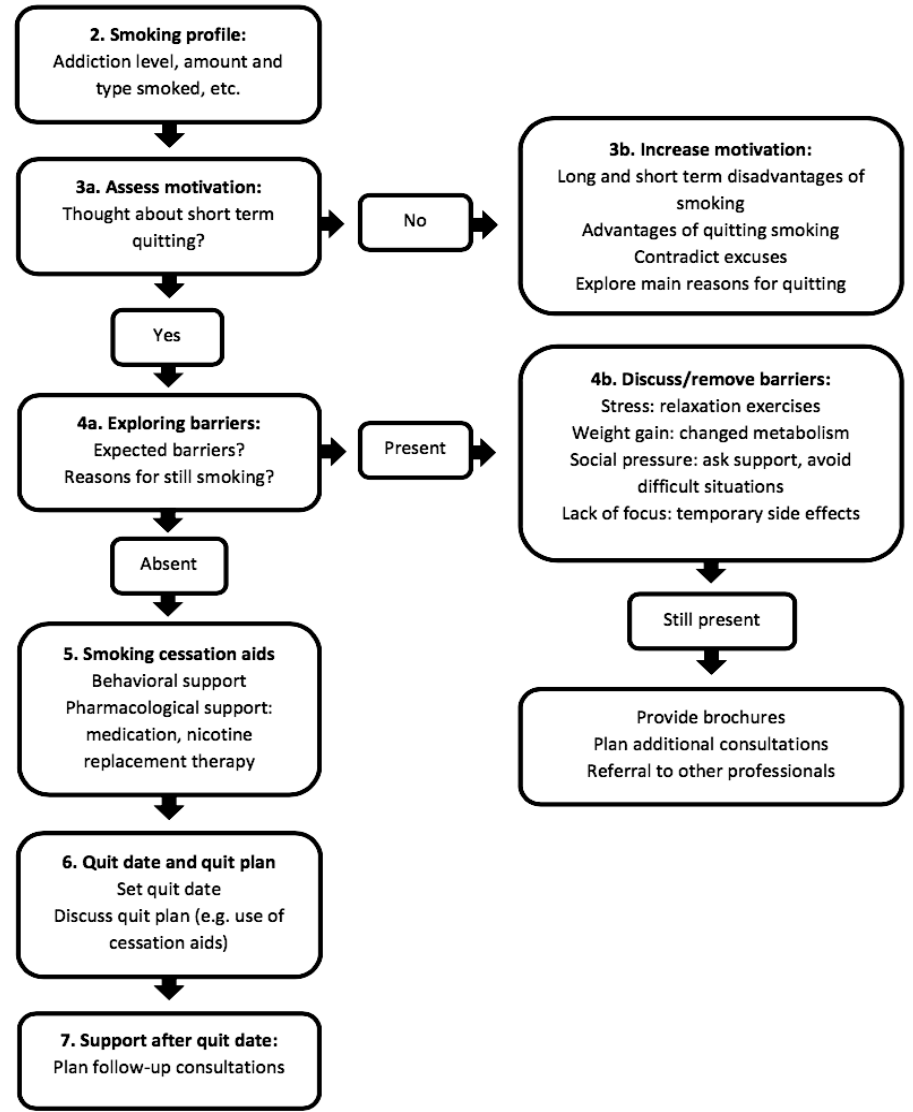

Figure 1 Counseling steps of evidence-based smoking cessation guidelines

1) Provide a quit advice. Each smoking patient visiting a healthcare professional should receive a brief advice to quit smoking. This is especially important, as research has demonstrated that such an advice alone could already help smokers to quit successfully (Stead et al., 2013). According to the guideline, a brief quit advice should at least be provided to patients with smoking-related complaints and to each new patient that smokes.

2) Assess smoking profile. The next step should be to assess the smoking profile (e.g. when and what is smoked) and smoking history (e.g. previous quit attempts made) of the patient. This will help the healthcare professional to paint a better picture of the smoking patient, enabling the professional to adjust cessation counseling to individual characteristics and the personal situation of the patient (e.g. a patient smoking two packs a day might benefit more from pharmacotherapeutic support compared to a patient only occasionally smoking at parties (Bader, McDonald, \& Selby, 2009)). 
3a) Assess motivation. Next, a patients' motivation to quit smoking needs to be explored to determine the feasibility of a short-term quit attempt. Often a six-month cutoff point is used to assess a patient's willingness to quit, in line with the stage-based approach towards behavior change of the Transtheoretical Model (Prochaska \& Velicer, 1997). Healthcare professionals are advised to adapt their counseling to the 'stage of change' of each individual patient and to guide a smoking patient through the subsequent stages until a successful quit attempt is made.

3b) Increase motivation. When a smoking patient is not (yet) motivated to quit, healthcare professionals should further explore where this lack of motivation originates from (i.e. assess perceived (dis)advantages of smoking and (dis)advantages of quitting). Motivation-enhancing strategies can then be applied, such as eliciting a main, personal reason for quitting (e.g. to prevent exposure to second-hand smoking of (grand)children) or contradicting a smoker's perceived disadvantages of quitting (e.g. quitting can indeed be expensive, but think of all the money for cigarettes that is saved).

4a) Explore barriers. When a patient feels motivated enough to engage in a shortterm quit attempt, the next step for the healthcare professional should be to explore a patient's potential barriers towards refraining from smoking. Often mentioned barriers are unwanted side effects such as weight gain or stress, and an unsupportive social environment (e.g. a smoking partner who is unwilling to quit).

4b) Discuss/remove barriers. Subsequently, healthcare professionals should discuss potential strategies to overcome identified barriers together with the patient, in order to provide patients with confidence that they can remain quit, even in difficult situations. For example, a smoking patient struggling to give up a cigarette that he usually pairs with a cup of coffee, could be advised to make a plan about replacing the coffee with a different beverage. This form of action planning could help to prevent smoking relapse (de Vries, Eggers, \& Bolman, 2013; Gollwitzer, 1999).

5) Discuss cessation aids. Next, different types of cessation support should be described to smoking patients in order to provide them with an overview of all possibilities concerning behavioral (e.g. group or individual counseling) and pharmacotherapeutic cessation support (i.e. medication such as varenicline or bupropion, and nicotine replacement therapy such as nicotine patches). Based on a patient's preferences and smoking profile, healthcare professionals should provide individually tailored recommendations (e.g. to fight withdrawal symptoms heavy smokers may benefit more from medication combined with nicotine replacement therapy, compared to less frequent smokers (Ebbert, Hays, \& Hurt, 2010)).

6) Help set a quit date and develop a quit plan. Based on decisions made while discussing the topics of all previous counseling steps, a quit plan should be developed next. As part of this plan, the patient and healthcare professional set a quit date and describe the methods that will be used to support the quit attempt.

7) Offer support after the quit date. Finally, follow-up consultations after the set quit date should be planned to monitor the smoking cessation process. These consultations 
will particularly address preventing smoking relapse (e.g. coping with not smoking in difficult situations) and should be scheduled up to six months after the quit date.

\section{Practice nurses' adherence to the smoking cessation guideline}

Little research has been conducted on PNs' adherence to the steps of the evidence-based smoking cessation guideline in the Netherlands. Therefore, an exploratory study among 50 Dutch PNs was conducted in 2012; findings from this telephone-based, self-report study showed that only $52 \%$ of PNs reported to provide smoking patients with a quit smoking advice and even less PNs (42\%) reported to fully adhere to all guideline steps (Smit, Hoving \& de Vries, not published). As participants from this exploratory study had previously participated in a smoking cessation research trial (Smit et al., 2012) they likely represent a rather dedicated group of PNs, very well aware of the current guideline and counseling practices. As such, the adherence rate of an average Dutch PN might be even lower than suggested by these preliminary findings. A more recent study in Dutch general practice seems to confirm this: only about a quarter of smoking patients visiting their general practice received a brief quit smoking advice from their GP or PN (Kleinjan, Bommelé, Verdurmen, \& van Laar, 2016). This is a missed opportunity to even apply the first guideline step, which should be a minimally time-consuming guideline element, to over two thirds of Dutch smokers that can be reached in general practice. Not providing a brief quit advice implies that - in line with preliminary findings by Smit and colleagues many patients likely also miss out on subsequent enrollment in structured smoking cessation counseling from a PN in general practice. In other words, adherence to the national evidence-based smoking cessation guideline seems to be less than optimal in Dutch general practice.

Adherence to an evidence-based smoking cessation counseling guideline is important as research has demonstrated that full application of all counseling steps has better effects on patients' quit rates than only a brief quit advice or less intensive counseling (Rice et al., 2017; Stead et al., 2013). However, even in situations when a PN does provide a brief quit advice, this does not always result in structured smoking cessation counseling. Research among American PNs showed that especially counseling steps that usually follow the provision of a quit advice, such as assessing quit motivation and informing about cessation aids, were less well adhered to than providing a quit advice (Studts et al., 2010; Walters et al., 2014). Ultimately this limits the effectiveness of PN-led smoking cessation counseling in general practice; a phenomenon that seems to be applicable to Dutch general practice as well. Moreover, since preliminary Dutch findings indicated sub-optimal guideline adherence by PNs and cessation support in Dutch general practice predominantly relies on nurse-led counseling, there seems to be considerable room for improvement concerning smoking cessation counseling by Dutch PNs. Yet, before attempts can be made to improve PNs' guideline adherence, more research is needed to obtain a de- 
tailed overview of their current counseling activities. Therefore, it is essential to investigate to what extent Dutch PNs adhere to all steps of the national evidence-based smoking cessation guideline and to explore what factors influence their guideline adherence.

\section{Determinants of practice nurses' guideline adherence}

Previous studies have identified various determinants that influence PNs' smoking cessation guideline adherence. These often concern practical factors such as time constraints (Halcomb et al., 2015; van Rossem et al., 2015), patient factors such as low smoker motivation to quit, or personal factors such as the belief that smoking cessation counseling is not an appropriate use of their time (Studts et al., 2010). Therefore, an intervention should ideally target multiple groups of determinants when aiming to promote change among primary care professionals (Lau et al., 2016; May et al., 2007). This idea is similar to the theoretical grounding of models that describe the dissemination and implementation of innovations in healthcare (Damschroder et al., 2009; Fleuren, Wiefferink, \& Paulussen, 2004). As the dissemination of a national smoking cessation guideline can be seen as the dissemination of an innovation, factors related to similar groups of determinants are likely to be important for promoting guideline adherence by PNs as well. For instance, the model by Fleuren and colleagues (2004) distinguishes between determinants from the socio-political context, and determinants of the organization, the innovation and the user.

External determinants that are related to the socio-political (e.g. rules and legislation) or organizational context (e.g. organizational size and hierarchical structure) can be particularly difficult to change in practice as they often result from top-down decisions by multiple stakeholders (Sangster-Gormley, Martin-Misener, Downe-Wamboldt, \& Dicenso, 2011). Yet, Dutch PNs are also facilitated to adhere to a smoking cessation guideline through external determinants. Within a socio-political context, national policy is in effect that ensures compensation for PNs to engage in smoking cessation counseling with their patients (Trimbos-instituut \& NHG, 2016), and within the organizational context (i.e. general practices) PNs are often facilitated with a place and timeslot, which is dedicated to smoking cessation counseling. Despite such external efforts to facilitate implementation of the national smoking cessation guideline, it is necessary to also invest in targeting determinants at the innovation and user level. Determinants related to the innovation might be rather easy to target, as innovation developers can alter the design and format of the innovation, based on the needs and preferences of the target group (Yardley et al., 2016). This would ideally result in an innovation with optimal usability characteristics concerning compatibility, complexity, trialability, observability and relative advantage (Rogers, 2003). Nevertheless, offering PNs a smoking cessation guideline that optimally matches their needs and preferences, still does not guarantee adequate guideline adherence, as also individual PN determinants will influence the decision to implement the guideline in practice. Individual PN determinants such as perceiving smoking cessation 
counseling as their responsibility can stimulate guideline adherence (van Rossem et al., 2015), whereas their perceptions of difficulty to integrate guideline use into daily practice can hinder guideline adherence (Halcomb et al., 2015). Investigating such individual determinants of PNs' guideline adherence is important in order to determine which determinants to target to facilitate implementation of the guideline in practice.

Previous research on determinants of nurses' smoking cessation support, for instance, demonstrated perceived advantages and support from the social environment towards using a counseling protocol to be important concerning the adoption and application of a smoking cessation protocol at Dutch cardiology wards (Berndt et al., 2013; Segaar, Willemsen, Bolman, \& De Vries, 2007). Also, a study among Dutch PNs revealed that a positive attitude (i.e. perceiving many advantages and little disadvantages) and positive social norms were important factors associated with the adoption of a new smoking cessation intervention (Leitlein, Smit, de Vries, \& Hoving, 2012). Regarding implementation of this same intervention, again a positive attitude towards the intervention proved to be an important socio-cognitive determinant (Smit, de Vries, \& Hoving, 2013). Such research is needed to obtain insight in which and to what extent individual socio-cognitive determinants influence PNs' smoking cessation support behavior, and to identify which of these determinants are important to target in an intervention. Nevertheless, insights into socio-cognitive determinants of guideline adherence have not yet been obtained among Dutch PNs, despite the fact that they are essential to understand and ultimately improve PNs' guideline adherence when counseling smoking patients in general practice. More research is therefore needed to obtain an overview of important socio-cognitive determinants of PNs' guideline adherence.

\section{The I-Change Model}

The systematic development of behavior change interventions has emerged with the introduction of the Precede-Proceed model (Green \& Kreuter, 1999). With this, especially the role of theory and evidence has become of particular importance in the field of planned health promotion. An important phase in planned health promotion is the investigation of determinants of the problem behavior, based on behavioral theories, in order to subsequently translate these into evidence-based intervention strategies and methods (Kok et al., 2016). This is recognized as an essential phase in planned health promotion as such systematic use of behavioral theory is considered to improve the effectiveness of behavioral change interventions (Davis, Campbell, Hildon, Hobbs, \& Michie, 2015; Fishbein \& Yzer, 2003; Glanz \& Bishop, 2010). More specifically, a theoretical behavior change model can be used to systematically identify the most important socio-cognitive determinants that influence PNs' smoking cessation guideline adherence, in order to develop a health promotion intervention that is both theory-based and evidence-based (Kok et al., 2016; Webb, Sniehotta, \& Michie, 2010). 
Therefore, studies reported on in this dissertation have been theoretically grounded in the I-Change Model (de Vries, 2017). Constructs from the I-Change Model have previously been used to successfully analyze the behavior of (practice) nurses (Bolman, de Vries, \& Mesters, 2002; Segaar, Willemsen, et al., 2007; Smit, de Vries, et al., 2013) and other healthcare professionals in relation to smoking cessation counseling (Berndt et al., 2013; Hoving, Mudde, \& de Vries, 2007; Segaar, Bolman, Willemsen, \& De Vries, 2007). These studies illustrate the applicability of the I-Change Model to investigate the influence of socio-cognitive determinants on the behavior of Dutch healthcare professionals, providing the rationale for the selection of this model to study PNs' smoking cessation guideline adherence in this dissertation.

The I-Change Model (Figure 2) is a behavior change theory which incorporates theoretical concepts from a number of socio-cognitive models (de Vries, 2017): the Transtheoretical Model (Prochaska \& Velicer, 1997), the Theory of Planned Behavior (Ajzen, 1985), Social Cognitive Theory (Bandura, 1986), the Health Belief Model (Janz, Champion, \& Strecher, 2002) and goal setting theories (Gollwitzer, 1999). The I-Change Model describes that the motivation to perform a (health) behavior is most proximally predicted by the motivational constructs attitude, perceived social influence and self-efficacy. According to the model, a person's attitude incorporates both the perceived advantages and disadvantages of the target behavior. Social influence relates to three separate concepts: social norms, which concern the perceived opinions of important others (e.g. family, friends, colleagues) with regard to the behavior; social modeling, which relates to the perceived behavior of these important others; and social pressure, which represents the perceived social pressure or support from important others to perform the behavior. A person's selfefficacy relates to the level of confidence in their ability to perform the behavior. When applying these constructs to PNs' smoking cessation guideline adherence, a positive attitude and self-efficacy towards using a guideline and a social environment which is perceived to approve of guideline use all contribute to a higher motivation to actually adhere to the national evidence-based guideline in practice.

Besides these motivational factors, the I-Change Model also includes awareness factors, information factors and preceding factors. Awareness factors include cognizance, knowledge, risk perceptions and perceived cues, which have a more distal influence on motivation (i.e. part of the pre-motivational stage). Information factors include personal (e.g. information seeking behavior), message (e.g. content matching with personal preferences), channel (e.g. web-based material) and source factors (e.g. from a trustworthy organization) related to the quality and modalities of information delivery. Preceding factors include biological (e.g. age, gender), psychological (e.g. personality traits), behavioral (e.g. lifestyle, past experiences with the target behavior) and environmental factors (e.g. socio-economic status, policies), which are external factors that may indirectly influence the motivational constructs and can also directly influence behavior. Finally, the I-Change Model also recognizes that intentions will not automatically be translated into behavior, a phenomenon also referred to as the intention-behavior gap, describing the discrepancy 
between one's motivation to change or perform a particular behavior and actual behavioral performance. In the action phase, factors such as self-efficacy, action planning, skills and barriers influence the likelihood of the translation of a positive intention into behavior. The first three factors can increase the likelihood of intention being transferred into action, whereas the perception of barriers towards change can have adverse effects and widen the intention-behavior gap. Moreover, as part of action planning within the IChange Model, both the development of preparatory plans and coping plans can be distinguished (Stanczyk, Bolman, Muris, \& de Vries, 2011). Applied to PNs' guideline adherence, one would develop preparatory plans in order to prepare for using a guideline during a smoking cessation consultation with a patient (e.g. consult an overview of guideline steps) and coping plans to increase chances of adequate guideline adherence in situations in which this is perceived to be difficult (e.g. when limited time is available).

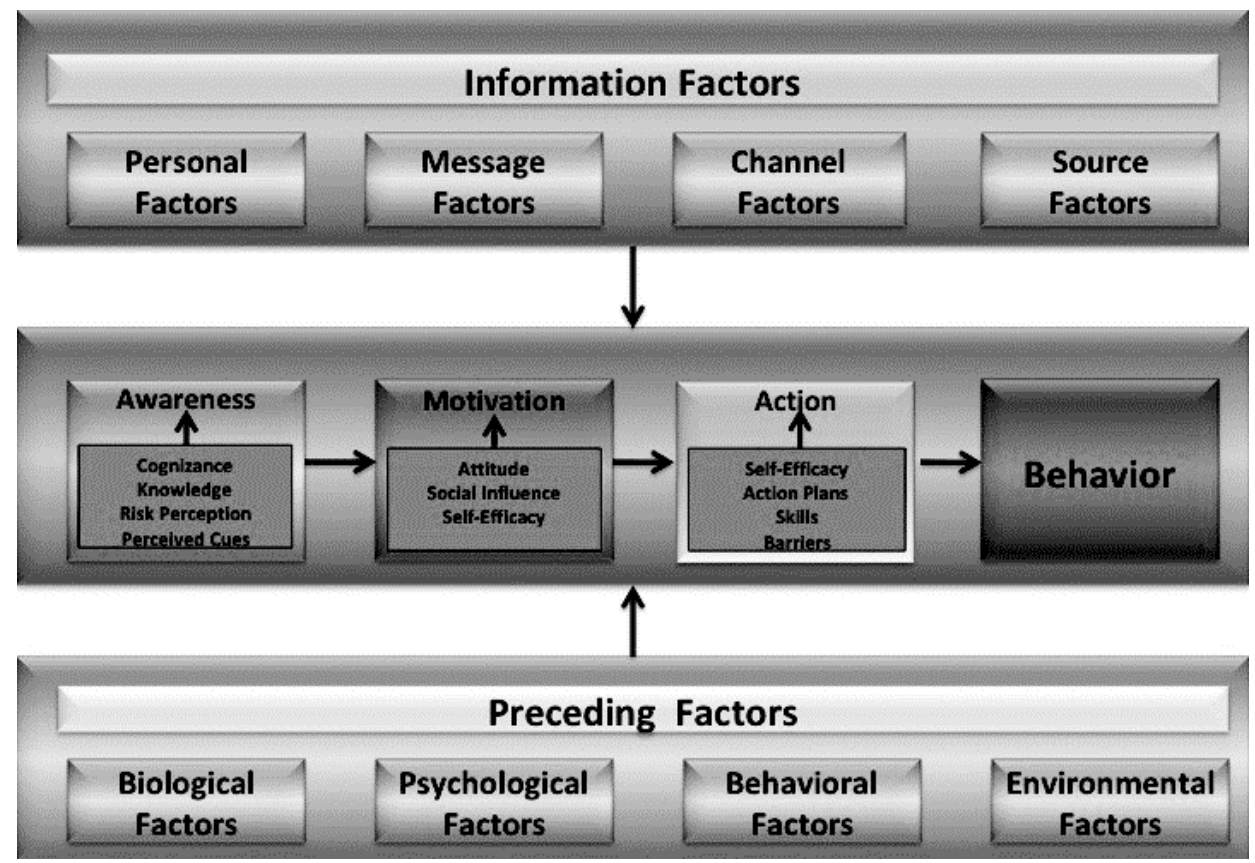

Figure 2 The I-Change Model (de Vries, 2017)

Understanding the influence of important socio-cognitive determinants on an individual's behavior (change) is essential to subsequently aim for improved behavioral performance by targeting these determinants in an intervention (Kreuter \& Wray, 2003). As it cannot be assumed that all PNs are motivated to change their guideline adherence behavior, it is particularly worthwhile to improve their motivation for behavior change. An effective way to do this is by targeting the most proximal determinants of motivation, which are an individual's attitude, perceived social influence and self-efficacy towards the target 
behavior. Previous studies, based on the I-Change Model (de Vries, 2017), have analyzed the behavior of (practice) nurses in relation to smoking cessation counseling and found that especially a positive attitude (Bolman et al., 2002; Segaar, Willemsen, et al., 2007; Smit, de Vries, et al., 2013) and a favorable social environment (Segaar, Willemsen, et al., 2007; Smit, de Vries, et al., 2013) were positively associated with PNs' counseling activities. Moreover, in a study among Dutch midwives, again their attitude and perceived social influence proved to be useful in explaining the uptake of smoking cessation counseling (Segaar, Bolman, et al., 2007), whereas a study among Dutch GPs and practice assistants additionally identified self-efficacy as an important determinant (Hoving et al., 2007). Furthermore, also more distal factors, such as awareness and knowledge, and factors concerning the action phase are often successfully targeted in tailored behavior change interventions. For instance, the level of knowledge of midwives about a smoking cessation protocol determined protocol adoption (Segaar, Bolman, et al., 2007) and making action plans via a web-based intervention was successful to promote smoking cessation (Bolman et al., 2015).

All of these studies illustrate the successful application of the I-Change Model to investigate the influence of various socio-cognitive determinants on the behavior of Dutch healthcare professionals. Hence, it is worthwhile to develop a behavior change intervention to improve PNs' smoking cessation guideline adherence by targeting specific sociocognitive determinants of their guideline adherence as derived from this theoretical behavior change model.

\section{Developing a tailored adherence support intervention}

\section{Tailoring intervention content on individual characteristics}

When developing an adherence support intervention for PNs one should not apply a 'one size fits all' principle to target important determinants of PNs' guideline adherence (Kreuter, Strecher, \& Glassman, 1999), as every individual PN's behavior is likely influenced by a different mix of important socio-cognitive determinants and personal beliefs. For instance, PNs' level of self-efficacy is likely to be subject to inter-individual variation as some PNs will already have sufficient self-efficacy to apply a smoking cessation guideline and are hence not in need of an adherence support intervention that aims to enhance their self-efficacy to use a guideline. On the other hand, PNs with a low level of self-efficacy, should be particularly reached with adherence support including content aimed to enhance their self-efficacy to use a guideline. This strategy of adapting intervention content to individual user characteristics is referred to as 'tailoring' (Kreuter et al., 1999). As similar inter-individual variation could be observed concerning other socio-cognitive determinants as well (e.g. different individuals perceiving many and few advantages of guideline use), this means that each individual PN would benefit from an intervention 
targeting a different mix of socio-cognitive determinants of guideline adherence (Hawkins, Kreuter, Resnicow, Fishbein, \& Dijkstra, 2008). Hence, by tailoring intervention content on socio-cognitive determinants an individualized intervention can be provided for each PN. Such a tailored intervention will likely be perceived as more relevant and will therefore be more carefully read and remembered, compared to generic, non-tailored interventions (Kreuter \& Wray, 2003).

Generic interventions often rely on strategies such as knowledge transfer (e.g. providing a guideline and supporting materials) and prompting (e.g. reminders to apply a guideline during a consultation with a patient), but do not incorporate inter-individual differences concerning PNs' socio-cognitive determinants of guideline adherence. Targeting these inter-individual differences can be achieved through tailoring (Kreuter et al., 1999). Tailored interventions are nowadays predominantly developed according to computertailoring principles, meaning that individually tailored advice is generated by computer software (i.e. previously this was done manually) that links specific user characteristics or beliefs (i.e. often assessed through a self-report questionnaire) to pieces of advice stored in a database (de Vries \& Brug, 1999). As such, computer-tailored programs can efficiently provide intervention content that is individually tailored to socio-cognitive determinants of a target behavior, such as PNs' smoking cessation guideline adherence. Tailored interventions have proven to be effective in changing various health behaviors and their determinants among different target populations (Krebs, Prochaska, \& Rossi, 2010) and have also proven to be successful in promoting the implementation of recommended practice (e.g. clinical guidelines) by a range of primary care professionals, including smoking cessation guideline adherence (Baker et al., 2015). For example, an intervention for physicians showed improved outcomes regarding adherence to a smoking cessation guideline (Unrod et al., 2007), but a similar intervention for PNs is still lacking (Baker et al., 2015). Moreover, in the study by Unrod and colleagues (2007), computer-tailored advice was provided via a brief print report, which likely caused a delay between filling out the study's baseline assessment and receiving the advice based on this assessment. Due to this delay, the perceived relevance of a print-based tailored message might be reduced, compared to a message that is received instantly after completing an assessment, for instance via a web-based channel (Krebs et al., 2010). Hence, providing PNs with instant tailored feedback on their guideline adherence via a web-based intervention could be a promising strategy to efficiently improve their guideline adherence.

\section{Web-based tailored interventions}

During the past decades, substantial progress has been made in developing tailored interventions in a more efficient and cost-saving way; whereas earlier computer-tailored interventions predominantly relied on print materials to spread tailored messages, they can nowadays easily be spread via web-based channels such as computers or mobile devices (Krebs et al., 2010). Integrating tailored advice in a web-based environment has 
therefore become an increasingly popular method for developing behavior change interventions (Kohl, Crutzen, \& de Vries, 2013; Lustria et al., 2013). Web-based interventions have several advantages for both provider and recipient. Many recipients can easily be reached, since Internet access is growing across the world enabling a large population (especially in North America (88.1\%) and Europe (77.4\%)) to use these interventions (Internet World Stats, 2017). As a result of the wide reach of web-based interventions, development and dissemination costs (per individual recipient) can remain relatively low. Next, users can consult the intervention wherever and whenever they find convenient, which makes it a suitable alternative for less flexible (e.g. face-to-face) interventions and may fit better in busy work schedules of PNs (Griffiths, Lindenmeyer, Powell, Lowe, \& Thorogood, 2006). Another advantage of web-based interventions is the fact that tailored feedback can be delivered in real-time (i.e. compared to delayed feedback of computertailored print interventions) to make sure the advice is still individually relevant to a PN's situation at the moment of receiving it. Finally, such interventions can mostly be offered in an anonymous way to ensure user privacy (Bennett \& Glasgow, 2009) and to protect users from being judged or stigmatized (Griffiths et al., 2006).

Previously tested web-based (tailored) interventions proved to be effective in changing various (determinants of) health behaviors (Taylor et al., 2017), also specifically among healthcare professionals (Sinclair, Kable, Levett-Jones, \& Booth, 2016). Webbased interventions to train or support healthcare professionals (i.e. often called e-learning) have for instance shown to be effective in improving the implementation of a suicide guideline in mental health care (de Beurs et al., 2015), adherence to an acute gastroenteritis guideline by physicians (Nicastro et al., 2015), nurses' knowledge level regarding a delirium (van de Steeg, IJkema, Wagner, \& Langelaan, 2015) and nurses' motivational interviewing skills (Fontaine et al., 2016). It is therefore hypothesized that a web-based computer-tailored intervention offering individually relevant advice (i.e. targeting sociocognitive determinants) on applying the national evidence-based smoking cessation guideline can effectively support PNs to improve their guideline adherence in general practice. It could, for instance, be possible to first ask PNs to fill out a web-based questionnaire concerning socio-cognitive determinants derived from the I-Change Model (e.g. self-efficacy to use a guideline in difficult situations or perceived advantages of using a guideline) and subsequently provide them with a web-based intervention containing tailored advice based on their answers. This computer-tailored advice could then provide feedback on potentially difficult counseling situations (e.g. when limited time is available) or specific benefits of using a smoking cessation guideline during consultations (e.g. increasing counseling quality), identified to be important by each individual PN. Up until now, such a web-based tailored intervention for PNs does not yet exist; hence to be able to support Dutch PNs to better adhere to the national evidence-based smoking cessation guideline, a novel web-based computer-tailored e-learning intervention for PNs (i.e. the STER intervention) was developed and tested. 


\section{STER intervention: e-learning for practice nurses}

\section{Systematic intervention development}

In order to develop an effective behavior change intervention, attention should be paid to characteristics such as rapid technological developments, a user-centered design, effective engagement and theoretical grounding (Michie, Yardley, West, Patrick, \& Greaves, 2017). First of all, technological developments have enabled intervention developers to administer interventions via digital channels or to integrate interventions in web-based platforms, meaning that the development of a novel intervention for PNs can benefit from adopting these technologies. Secondly, to realize an intervention with a user-centered design (i.e. a design that incorporates needs, values and preferences of the user) it is important to involve the target population (i.e. PNs engaged in smoking cessation counseling in Dutch general practice). Theories on systematic intervention development, such as Intervention Mapping, often call this the 'needs assessment' phase of intervention development, as researchers explore the needs of the target population in order to develop an intervention that optimally matches their needs (Kok et al., 2016). Thirdly, involvement of PNs in the development of an intervention will also make effective engagement (i.e. level of engagement with the intervention, sufficient to achieve intended outcomes) with the intervention a greater likelihood, as potential barriers and facilitators of intervention engagement can be explored beforehand (Yardley et al., 2016). Fourthly, ensuring theoretical grounding should also be incorporated in systematic intervention development. This is often done by conducting a thorough analysis of the target behavior and its associated behavioral determinants, based on one or more behavioral theories (Kok et al., 2016).

To systematically develop the STER intervention, a web-based computer-tailored elearning program for PNs, it is important to first conduct formative research among PNs. During the needs assessment phase, it is essential to gain a deeper understanding of PNs' current smoking cessation counseling activities in general practice: how does the enrollment of patients in smoking cessation counseling take place and how well do PNs apply the national guideline during patient consultations? Also, specific factors facilitating and hindering PNs' application of a smoking cessation guideline need to be explored in order to develop an intervention with a user-centered design, which is compatible to current practice. For this purpose, also PNs' preferences regarding the type of content (e.g. text, image, video, etc.) and the design of the intervention (e.g. interactive elements, help section, log-in procedure, etc.) should be explored to ensure the development of an intervention that stays close to the preferred user experience of PNs (Yardley et al., 2016). For example, if PNs report to struggle with time constraints during office hours, this could be taken into consideration by developing an intervention with 24/7 accessibility or with content that is divided into small segments (i.e. to enable PNs to consult it in a more 
flexible way than an extensive text). Such strategies will increase the odds of PNs' (repeated) use of the intervention (Brouwer et al., 2011; Rogers, 2003) and will make effective engagement a stronger likelihood. Therefore, in chapter 2 of this dissertation the results of a qualitative needs assessment among PNs will be described, exploring PNs' current counseling practices, hindering and facilitating factors associated with their adherence and their needs regarding the STER intervention.

Next, a detailed analysis of PNs' (determinants of) smoking cessation guideline adherence should be explored, informed by theoretical constructs from the I-Change Model (de Vries, 2017). First, by analyzing PNs' application of each individual guideline step (i.e. step-based adherence) detailed insight will be obtained about their adherence, which can inform decisions about guideline steps that should receive more attention in an intervention because they are less well adhered to than other guideline steps. Secondly, the influence of socio-cognitive determinants, such as PNs' attitude towards and self-efficacy of using a guideline, on PNs' (step-based) adherence should be investigated. By investigating such determinants also for each guideline step separately, an extra dimension can be added to the advice for PNs: advice can be tailored to an individual PN's score on the socio-cognitive determinants of adhering to every individual step of the guideline. For example, tailored advice can be provided to a PN with low self-efficacy to provide a smoking patient with a brief quit advice. Being able to develop such detailed tailored messages with more individually relevant and personalized content will likely stimulate PNs' interest towards their advice, which should lead to more careful reading and remembering of the content (Kreuter \& Wray, 2003). Therefore, the results of a web-based cross-sectional study among PNs will be described in chapter 3 of this dissertation, providing a detailed overview of PNs' (step-based) guideline adherence and important determinants of their adherence.

After intervention development, target group involvement remains important as developers need to test whether the intervention works as it is supposed to (Nielsen \& Mack, 1994). For this purpose, the intervention's usability needs to be reviewed, preferably by both members of the target population (i.e. usability tests) and by intervention or behavior change experts (i.e. expert usability review). The goal of conducting usability tests and reviews is to reveal issues with intervention use in practice and to subsequently solve these issues before the intervention is evaluated in a research trial or made available for public use (Krug, 2006). Concerning the STER intervention, systematic intervention development and usability review ultimately resulted in a computer-tailored e-learning program for PNs, consisting of 1 ) three modules with computer-tailored advice aimed to improve (determinants of) PNs' guideline adherence, 2) an online forum to get in touch with other PNs, and 3) a module with practical counseling information to inform both PNs and counseled patients. A more detailed description of the development of the STER intervention and its different components is described in chapter 4 of this dissertation. 


\section{Intervention evaluation}

After systematic development of an intervention, it is usually evaluated in terms of its (costleffectiveness and appreciation by its users (Kok et al., 2016). To evaluate an intervention's effectiveness, it is considered to be the golden standard to conduct a randomized controlled trial (RCT), in which participants are randomly allocated to either an intervention group or control group and subsequently compared regarding a selected outcome measure or target behavior (Campbell et al., 2000; Flay, 1986). Interventions in the field of health promotion have the tendency to consist of multiple interconnecting parts, so-called 'complex interventions', as changing health behavior often includes multiple stakeholders and approaches (Flay, 1986). As a result, researchers often need to make well-balanced decisions when conducting an RCT concerning a health promotion intervention. For instance, a decision needs to be made about what 'treatment' control group participants will receive during the trial; it may not be acceptable that control group participants do not receive treatment at all (i.e. no exposure to the intervention) and not feasible to provide a placebo treatment (i.e. exposure to the intervention without any effective components), as is usually the case in RCTs of drug treatment (Campbell et al., 2000). Instead, control group participants in RCT's evaluating a health promotion intervention can receive an alternative treatment or treatment as usual (i.e. exposure to a different intervention), or delayed treatment (i.e. exposure to the intervention after trial completion, a so-called waiting list control group). As exposure to treatment as usual can be as complex as the intervention itself, careful monitoring of care in both groups is advised (e.g. patients' evaluation of received care), as well as an evaluation of user appreciation (e.g. program's usefulness and user-friendliness) in the intervention arm of the trial. Also, generalizability of RCT findings can be improved by ensuring that an intervention is evaluated in the same setting in which it will likely be implemented (Campbell et al., 2000). Concerning evaluation of the STER intervention this means that the intervention's effects on PNs' smoking cessation guideline adherence need to be evaluated in Dutch general practices, while comparing its effects against PNs' usual smoking cessation care. The results of this evaluation, an RCT testing the effectiveness of the STER intervention, are described in chapter 5 of this dissertation.

Within this RCT, PNs in the control group did not have access to the STER intervention but only provided usual smoking cessation care. As it is known that PN-led smoking cessation counseling in itself can already be effective to supports smokers to quit (Rice et al., 2017) and it is hypothesized that the STER intervention could further improve PNs counseling, it is important to also evaluate whether providing PNs access to the STER intervention can improve quit rates among their counseled patients. In order to evaluate patients' smoking behavior, data on smoking abstinence was collected from patients counseled by PNs in both the control and intervention group of the RCT concerning the STER intervention's effectiveness. The results of this evaluation of the STER intervention concerning effects on counseled patients' abstinence rates are described in chapter 6 of this dissertation. 
Evaluation of the effects of the STER intervention on PNs' guideline adherence and on patients' quit rates, will demonstrate the intervention's potential to have an important public health impact by reducing smoking prevalence as well as smoking-related illness among Dutch (ex-)smokers (WHO, 2017). However, intervention impact is also determined by its implementation in practice (Glasgow, Vogt, \& Boles, 1999) and hence influenced by the decision of policy makers to invest in intervention implementation. Nowadays, such decisions concerning health-related policy and resource allocation are often and ideally - informed by economic evaluation studies (Evers, Wolfs, \& Van Heugten, 2010). In an economic evaluation, besides intervention effects, also an intervention's costs are assessed in order to calculate a so-called cost-effectiveness ratio. The more effective an intervention is and the less costs are associated with these effects, the better this cost-effectiveness ratio. As policy makers often need to choose between interventions (e.g. different smoking cessation intervention, or even interventions from different health domains), information about the cost-effectiveness ratio of each intervention should be used to inform their decision (Evers et al., 2010). To facilitate intervention comparison, it is essential that more economic evaluation studies of interventions are conducted in the field of health promotion. Recent trials have increasingly also included an analysis of cost-effectiveness, for instance concerning smoking cessation interventions for patients (Smit, Evers, de Vries, \& Hoving, 2013; Stanczyk, Smit, et al., 2014) and also for healthcare professionals (Cantor et al., 2015; Pinget, Martin, Wasserfallen, Humair, \& Cornuz, 2007). Yet, the interventions for healthcare professionals tested so far consisted of face-to-face (as compared to web-based) training programs and did not specifically target PNs. This means that an economic evaluation of the STER intervention will produce novel findings on the cost-effectiveness of a web-based computer-tailored e-learning intervention for PNs. Therefore, the results of this economic evaluation are presented in chapter 6 of this dissertation.

\section{Aim and outline of the dissertation}

The present dissertation aims to provide detailed insight in the current smoking cessation counseling practices of PNs in Dutch general practice and the (cost-)effectiveness of the STER intervention, a web-based computer-tailored e-learning program aimed to improve PNs' adherence to the national evidence-based smoking cessation guideline. The subsequent chapters of this dissertation will together contribute to this overall aim.

Chapter 2: A qualitative needs assessment aimed to explore PNs' current counseling practices, hindering and facilitating factors associated with their adherence to the national evidence-based smoking cessation guideline and their needs regarding the STER intervention. 
Chapter 3: A web-based questionnaire study aimed to determine the association between socio-cognitive and predisposing factors, and PNs' overall and step-based adherence to the national evidence-based smoking cessation guideline.

Chapter 4: The development of the STER intervention and the study design of the trial testing the intervention's (cost-)effectiveness.

Chapter 5: Results of an RCT testing the effectiveness of the STER intervention on PNs' evidence-based smoking cessation guideline adherence.

Chapter 6: A trial-based economic evaluation of the STER intervention, assessing both its cost-effectiveness and cost-utility.

Finally, chapter 7 of this dissertation contains a general discussion of all chapters and the implications for practice and further research. 

Chapter

\begin{abstract}
Understanding Dutch practice nurses' adherence to evidence-based smoking cessation guidelines and their needs for web-based adherence support:
\end{abstract} results from semistructured interviews 


\section{Abstract}

Objectives: Practice nurses in general practices sub-optimally adhere to smoking cessation guidelines. As the effectiveness of their smoking cessation support is greatest when full adherence to these guidelines is achieved, interventions need to be developed to improve practice nurses' guideline adherence, e.g. by tailoring their content to adherence determinants. However, the socio-cognitive determinants explaining adherence have not yet been investigated. Therefore, this qualitative needs assessment aimed to explore not only practice nurses' current counseling practices, but also their socio-cognitive beliefs related to their smoking cessation guideline adherence and their needs regarding webbased adherence support.

Setting: primary care; general practices in the Netherlands.

Participants: nineteen practice nurses, actively involved in smoking cessation counseling.

Methods: Semi-structured individual interviews, based on the I-Change Model and The Diffusion of Innovations Theory, were conducted in May-September 2014. Data was systematically analyzed using the Framework Method and considered reliable (Kappa 0.77; percent agreement 99\%).

Results: Respondents felt able to be empathic and collaborative during smoking cessation consultations. They also reported psychological (e.g. low self-efficacy to increase patient motivation and arranging adequate follow-up consultations) and practical barriers (e.g. outdated information on quit support compensation and a perceived lack of high quality trainings for practice nurses) to smoking cessation guideline adherence. Most respondents were interested in web-based adherence support to overcome these barriers.

Conclusions: Socio-cognitive determinants influence practice nurses' smoking cessation guideline adherence. To improve their adherence, web-based tailored adherence support can provide practice nurses with personally relevant feedback tailored to individually perceived barriers to smoking cessation guideline adherence. More specifically, low selfefficacy levels can be increased by peer modelling (e.g. presenting narratives of colleagues) and up-to-date information can be presented online, enabling practice nurses to use it during patient consultations, resulting in more effective communication with their smoking patients. 


\section{Introduction}

Globally, smoking continues to be the leading cause of preventable disease and premature death (USDHHS, 2014; WHO, 2013). As 23\% of the Dutch adult population still smokes (RIVM, 2014) successful smoking cessation strategies are needed. Advice and counseling by a general practitioner (GP) is a cost-effective strategy to increase patients' quit rates (Stead et al., 2013). However, a shift in cessation counseling can be observed, where it is increasingly provided by trained practice nurses (PNs) in Dutch general practices (Freund et al., 2015; Nederlands Huisartsen Genootschap \& Landelijke Huisarsten Vereniging, 2011). PNs are predominantly responsible for chronic patient care and lifestyle counseling, applying evidence-based guidelines. PNs are highly educated (i.e. college degree) and employed in $80 \%$ of Dutch general practices (Heiligers et al., 2012).

Regarding smoking cessation, PNs are trained to use one of several national smoking cessation guidelines that include similar counseling steps (Figure 1) and of which the STIMEDIC ${ }^{\circledR}$ guideline is the most recent (Stop Smoking Partnership, 2009). In the Netherlands, the 'Stop Smoking Partnership', an alliance of several parties involved in smoking cessation in the health care sector, is responsible for developing and regularly updating national smoking cessation guidelines. These guidelines can be used by health care professionals to structure consultations with smoking patients and full adherence to these evidence-based guidelines leads to more effective health communication and hence positively contributes to quality of smoking cessation care (Miller \& Kearney, 2004; Woolf, Grol, Hutchinson, Eccles, \& Grimshaw, 1999). Adequate guideline adherence is also known to have stronger effects on patients' quit rates than a brief quit advice (Rice, Hartmann-Boyce, \& Stead, 2013).

However, PNs regularly report suboptimal adherence to smoking cessation guidelines (Glasgow, Lichtenstein, \& Marcus, 2003; Segaar, Willemsen, et al., 2007; Whitehead, Zucker, \& Stone, 2014), due to practical factors such as time constraints or the perception that most smoking patients are unwilling to quit (Studts et al., 2010; van Rossem et al., 2015; Whitehead et al., 2014). Moreover, research has especially shown that socio-cognitive determinants consistently influence guideline adherence among health care professionals. A study among cardiac nurses working in a hospital (Bolman et al., 2002) found significant positive correlations between self-efficacy expectations, the perceived simplicity and advantages of the guideline, and intention towards continued use of a smoking cessation guideline. Relatedly Segaar and colleagues (2007) concluded that a positive attitude, positive social influences and higher self-efficacy best explained adoption of the same guideline by cardiac nurses. Though these studies illustrate the importance of sociocognitive determinants in explaining smoking cessation guideline adoption and adherence, such data are not available for PNs working in general practices. It might be that different determinants influence the behavior of PNs as they have different types of training and expertise, and work in a different setting with other types of patients compared 
to cardiac nurses. Therefore, it is important to investigate PNs' current counseling practices and the role of socio-cognitive determinants in explaining their smoking cessation guideline adherence in a general practice setting.

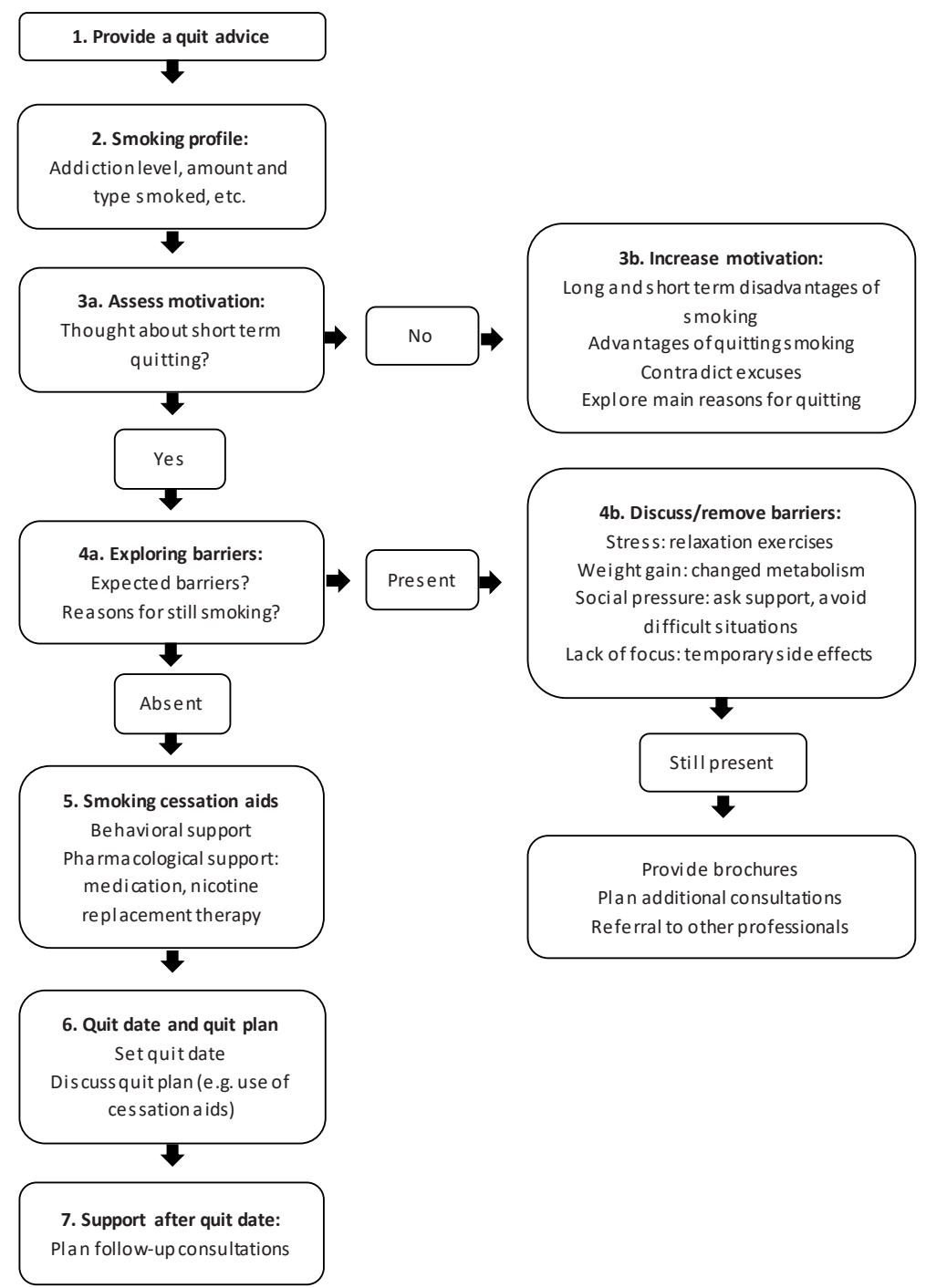

Figure 1 Flow chart Dutch smoking cessation guidelines (adapted from Guideline treatment tobacco addiction revision 2009 (Stop Smoking Partnership, 2009))

Web-based computer tailored (CT) programs have been shown to be able to effectively change various health behaviors and socio-cognitive determinants among the general population and patient groups (Civljak, Stead, Hartmann-Boyce, Sheikh, \& Car, 2013; Kohl et al., 2013; Krebs et al., 2010; Lustria et al., 2013). CT programs provide content adapted 
to characteristics (e.g. demographic factors and socio-cognitive determinants) of the individual user (Kreuter \& Wray, 2003). A CT program can, for instance, provide an individual with personal feedback about their behavior, which is based on that individual's answers to a questionnaire. Consequently, individually tailored content is personally relevant and more likely to be read and remembered compared to more static tools such as leaflets or generic websites (Kreuter \& Wray, 2003). Moreover, through web-based support the intervention elements can be provided when and where PNs require them at a relatively low cost (Griffiths et al., 2006; Tate, Finkelstein, Khavjou, \& Gustafson, 2009). Offering a minimally time-consuming approach enables PNs to integrate adherence support in their busy schedules, which was found to be important when asking nurses regarding their preferences for smoking cessation training (Katz et al., 2014; Smith, Sellick, \& Spadoni, 2012). However, CT support has only been sparsely applied to increase smoking cessation guideline adherence among health care professionals and none have been offered in a web-based format. One study assessed the use of printed CT advice to increase smoking cessation counseling (Unrod et al., 2007), demonstrating improved guideline implementation by physicians and increased patients' quit rates six months post-intervention. Despite these promising results of printed CT advice among physicians, to date neither printed nor web-based CT support exists to stimulate smoking cessation guideline adherence among PNs.

To develop a web-based CT adherence support program for PNs, it is essential to assess PNs' current counseling practices and how determinants influence their adherence to smoking cessation guidelines, and to what extent. Furthermore, PNs' needs regarding the content and design of web-based support need to be assessed to ensure compatibility with their daily practice, as this contributes to higher exposure to an intervention's content (Schneider, van Osch, \& de Vries, 2012). Therefore, we describe a qualitative needs assessment aimed to explore PNs' current counseling practices, the socio-cognitive determinants of their smoking cessation guideline adherence and their needs regarding web-based adherence support.

\section{Methods}

\section{Study design}

Data were gathered by means of semi-structured interviews with individual PNs ( $N=19)$, because individual interviews yield more in-depth information than group interviews (Bourgeault, Dingwall, \& Vries, 2010). PNs provided oral informed consent. Interview recordings and verbatim transcriptions were anonymized and stored in a password-protected database. Evaluation of this study by the Medical Ethics Committee Atrium-Orbis- 
Zuyd (14-N-17) revealed that no medical ethical clearance for this study was needed according to the rules of the Medical Research Involving Human Subjects Act (WMO). The study is registered with the Dutch Trial Register (NTR4436).

\section{Participants and procedure}

Dutch PNs, actively engaged in smoking cessation counseling in a general practice, were recruited via telephone between May and September 2014. We invited a heterogenic sample of PNs to obtain diverse responses to the interview questions. Through purposeful sampling we included PNs from a) different geographical regions in the Netherlands; b) different types of practices (urban and rural); and c) practices with different sizes (in terms of patients and employees). Furthermore, recruited PNs differed in their d) use of smoking cessation guidelines; e) amount of working experience; and f) educational level. Recruitment continued until data saturation was reached; PNs did not introduce any new issues during the interviews at this point. Successfully contacted PNs who declined to participate $(n=7)$ mentioned a lack of time and interest in the study as main reasons. All participating PNs were rewarded with a €10 gift card.

Interviews were conducted at the PNs' workplace $(n=17)$ or via telephone $(n=2)$ and only the PN and one or two researchers (i.e. DdR and LG) were present. No prior relationship between PNs and researchers was established before conducting the interviews. Both researchers had prior experience with conducting individual (i.e. DdR) and group interviews (i.e. LG). Before commencing the interviews, PNs were informed about the aims of the interview. Interviews were audio-recorded and notes were taken to keep track of the issues and themes that were covered during each interview. Interviews lasted for 25-62 minutes ( $m=43$ minutes).

\section{The interview guide}

A semi-structured interview guide was developed based on the I-Change Model (de Vries et al., 2003) and The Diffusion of Innovations Theory (Rogers, 2003), a combination which has shown to be successful in explaining the implementation of smoking cessation interventions by primary health care professionals (Segaar, Willemsen, et al., 2007; Smit, de Vries, \& Hoving, 2010). In the interview guide follow-up questions relating to PNs' current counseling practices and prompt questions regarding the STIMEDIC ${ }^{\circledR}$ guideline were especially informed by the concepts attitude, social influence, self-efficacy, perceived barriers and skills from the I-Change Model (de Vries et al., 2003). Questions relating to PNs' needs for web-based support (i.e. the innovation) were guided by Rogers' five innovation characteristics: the relative advantage, compatibility, complexity, trialability and observability of the innovation (Rogers, 2003). Two primary care professionals engaged in smoking cessation counseling pre-tested the interview guide, which was adapted according to their feedback. The final interview guide (Appendix 1) consisted of open-ended questions 
and prompts to encourage PNs to discuss their current smoking cessation practices, guideline adherence and desired program characteristics.

Interviews were started with an assessment of PNs' personal (age, gender, personal smoking history and professional education) and work-related characteristics (years of experience as $\mathrm{PN}$, the number of practices employed in, and the size, location and type of the(se) general practice(s)). Subsequently, PNs were asked to describe their smoking cessation counseling routine and discuss barriers encountered during counseling. Thirdly, the STIMEDIC ${ }^{\circledR}$ smoking cessation guideline was introduced and PNs were asked to identify guideline steps that they would find difficult to perform and to describe situations they might find difficult to handle when applying these steps. Finally, PNs' needs were explored, including interest in and preferences regarding the content and design of webbased adherence support aiming to improve their smoking cessation counseling.

\section{Data analysis}

Data analysis using the Framework Method (Gale, Heath, Cameron, Rashid, \& Redwood, 2013) was conducted using Nvivo (version 9). First, interviews were transcribed verbatim and two coders (i.e. DdR and LG) familiarized themselves with the interview content by listening to audio records of the interviews and reading transcripts. Next, both coders independently developed a coding tree by analyzing the same single transcript and the coding trees were compared for consistency (Campbell, Quincy, Osserman, \& Pedersen, 2013); any inconsistencies were discussed and resolved. Then, three similar rounds of independent coding of three additional transcripts resulted in a final coding tree consisting of multiple major and minor themes for every main interview question. This coding tree was perceived to cover all relevant information and agreed upon by both coders. Subsequently, both coders independently applied the coding tree to the same five randomly selected interview transcripts and the intercoder reliability was assessed, resulting in a value for percent agreement and Cohen's Kappa. Percent agreement reflects the degree of similarity between coders in assigning the same code to the same piece of text (Campbell et al., 2013) and a coefficient of $\geq 90 \%$ is considered acceptable (Krippendorff, 2004; Lombard, Snyder-Duch, \& Bracken, 2002). Cohen's Kappa, takes into account that agreement between coders might occur due to chance and is therefore a more conservative coefficient (Lombard et al., 2002); a Kappa of $\geq 0.70$ is considered acceptable (Lombard et al., 2002). It was determined that the intercoder agreement was sufficiently high (percent agreement 99\%; Cohen's Kappa 0.72). Nonetheless, coding inconsistencies were again discussed and resolved. Thereafter, the first coder (i.e. DdR) completed coding the remaining transcripts $(n=14)$ whereas the second coder (i.e. $L G)$ only coded the final $10 \%$ of the transcripts $(n=2)$. This revealed an improved intercoder reliability of $99 \%$ (percent agreement) and 0.77 (Cohen's Kappa). Once all transcripts were coded, themes were grouped together and data were clustered based on their importance. Finally, data 
clusters were interpreted by looking for patterns and identifying answers to the research questions.

\section{Results}

Participating PNs were on average 46.1 years old and mostly female; the majority had never smoked (Table 1). All PNs had obtained a Bachelor's degree in nursing, while some were additionally trained in pulmonary or diabetic patient care. PNs' mean work experience was 8.3 years, they were mostly employed at urban practices and reported a mean number of 8.4 employees (range 4-20) and a patient population of nearly 4,500 patients (range 500-12,000) per practice.

Table 1 Descriptive characteristics of participating PNs ( $N=19)$

\begin{tabular}{|c|c|c|}
\hline \multicolumn{3}{|l|}{ Personal characteristics } \\
\hline Mean age in years (SD) & 46.1 & $(9.2)$ \\
\hline Female (\%) & 17 & $(89.5 \%)$ \\
\hline $\begin{array}{l}\text { Smoking status } \\
\text { Never smoked (\%) } \\
\text { Ex-smoker }(\%)\end{array}$ & $\begin{array}{l}11 \\
8\end{array}$ & $\begin{array}{l}(57.9 \%) \\
(42.1 \%)\end{array}$ \\
\hline $\begin{array}{l}\text { Professional education } \\
\text { Post-bachelor PN training (\%) } \\
\text { Specialized diabetes nurse (\%) } \\
\text { Specialized pulmonary nurse (\%) }\end{array}$ & $\begin{array}{l}19 \\
3 \\
4\end{array}$ & $\begin{array}{l}(100 \%) \\
(15.8 \%) \\
(21.1 \%)\end{array}$ \\
\hline Mean working experience as $P N$ in years (SD) & 8.3 & $(4.0)$ \\
\hline \multicolumn{3}{|l|}{ Practice characteristics } \\
\hline Urban practice location (\%) & 12 & $(63.2 \%)$ \\
\hline Mean number of employees in practice(SD) & 8.4 & $(4.4)$ \\
\hline Mean patient visits per year* (SD) & 4478.4 & $(3016.4)$ \\
\hline Counseling elements & \multicolumn{2}{|c|}{ Adherence by PNs $(n)^{* *}$} \\
\hline Provide a quit advice & 10 & $(52.6 \%)$ \\
\hline Assess patients' smoking profile & 18 & $(94.7 \%)$ \\
\hline Assess patients' motivation & 18 & $(94.7 \%)$ \\
\hline Increase patients' motivation & 7 & $(36.8 \%)$ \\
\hline Asses patients' barriers & 15 & $(78.9 \%)$ \\
\hline Remove patients' barriers & 7 & $(36.8 \%)$ \\
\hline Discuss use of cessation aids & 16 & $(84.2 \%)$ \\
\hline Develop a quit plan & 16 & $(84.2 \%)$ \\
\hline Follow-up after the quit date & 18 & $(94.7 \%)$ \\
\hline
\end{tabular}

* Standardized practice capacity: 2168 patients per fulltime general practitioner

** The data represent the number (and percentage) of adherent PNs 


\section{Current smoking cessation counseling practices}

All PNs reported using a national guideline to structure their counseling, but described varying levels of guideline adherence. Smoking cessation counseling would usually be initiated by GPs' patient referral to a PN (i.e. GP provides a short quit advice, then refers to PN) or by PNs themselves giving patients a brief quit advice, especially 'people that visit with lung problems or diabetes'.

After a quit advice, additional counseling was predominantly conducted by PNs, mostly by first assessing their patients' smoking profile (e.g. amount smoked, smoking pattern, addiction level) during an intake interview.

Most PNs also reported to assess patients' motivation to quit, but more than half indicated to discontinue counseling if 'they [patients] are not internally motivated... Becoming internally motivated often takes longer than quitting itself.' Those PNs attempting to actively increase their patients' motivation used techniques like reflecting on patients' intrinsic reasons for quitting and providing patients with homework assignments.

'When they go home I ask [the patient] to write down their personal pros and cons of smoking... When they come back we especially discuss alternatives for the pros of smoking.'

The majority of PNs reported to assess patients' barriers for quitting, but less than half also attempted to remove present barriers, which was usually done by discussing distraction strategies to deal with craving.

'Craving for cigarettes only lasts a minute... I especially discuss distraction strategies to deal with this minute.'

Most PNs discussed the use of cessation aids (e.g. nicotine replacement therapy, medication, group counseling); especially focusing on their working mechanisms and possible side effects. Patients' preferences often influenced the decision to prescribe a particular cessation aid.

'Most patients know about Champix (varenicline) and specifically request it because they have heard about it from others.'

Subsequently, the majority of PNs reported to develop a quit plan with their patients, including a quit date, the use of cessation aids and follow-up appointments. They described that the duration, frequency and format of follow-up appointments after the quit date strongly depends on patients' preferences and their level of motivation, ranging from multiple face-to-face appointments to a single telephone appointment.

'First, patients often did not return... By planning follow-up appointments more frequently... I have more success in [helping them] quitting smoking.'

Concerning their role as a smoking cessation counselor, PNs perceived themselves able to 'adapt counseling to a [patient's] personal situation', to stimulate patients to make 
their own decisions by weighing personal pros and cons, and to provide patients with relevant information 'which is evidence-based' about the disadvantages of smoking.

\section{Experienced barriers during counseling}

PNs found it difficult to handle unmotivated patients, because they struggle to decide how much they should try to increase their patients' motivation. As they believed that the success of their counseling mainly depends on their patients' level of knowledge of and awareness about possibilities for smoking cessation and not so much on their own efforts to enhance patients' motivation, PNs sometimes decided to only provide patients with awareness-enhancing information.

'If a patient reports not to need counseling, I experience that it [counseling] has no effect. I am glad when they [patients] agree to take home some information.'

Several PNs described that this often happens after GPs directly referred patients without first assessing patients' motivation or providing a brief quit advice. They believed GPs' advice could be motivation-enhancing for patients, but felt 'it [GPs' quit advice] does not happen enough'.

If the counseling process was continued, PNs did not attempt to remove patients' barriers when their patients' motivation was low, as they perceived that 'unmotivated patients should first work on their motivation themselves'. PNs generally believed that first a sufficient level of motivation is needed (before dealing with the presence of barriers), but that achieving this is mainly the patient's own responsibility.

Regarding follow-up after the quit date, PNs felt smoking cessation guidelines lack detailed instructions on which topics to discuss during follow-up appointments.

'You call them [patients] or they visit the practice and of course you talk about their quit attempt, but there is no specific plan'

Moreover, they described that patients do not always perceive the need for follow-up and regularly skip or cancel scheduled appointments, making it difficult for PNs to 'get them [patients] to return to practice' for follow-up and to determine their success rate, since 'it is their [the patients'] own responsibility'.

Several PNs reported difficulties staying up-to-date regarding smoking cessation counseling as they perceived low supply of high-quality training classes. Furthermore, associated monetary and time investments were considered barriers for attending classes that do exist. Moreover, most PNs reported troubles with staying informed about compensation of counseling by different health insurances, as they all have their own regulations (e.g. a maximum number of consultations), which change regularly. Several PNs reported that 'online medication orders are not delivered on time', which means that ' $a$ well-prepared quit attempt falls to pieces'. Obtaining reliable information about both 
health insurances and ordering online prescriptions was described as 'very time-consuming and sometimes leading to confusion'.

Barriers for providing patients with educational materials included a lack of time and high-quality brochures, containing educational and visually attractive content. Moreover, patients were perceived as often not using provided materials and consequently lacking awareness of and knowledge about smoking cessation aids and counseling.

'It is not the case that you take a single pill of Champix and suddenly do not like cigarettes anymore... Sometimes it is necessary to adjust this expectation.'

\section{Needs regarding adherence support}

Most PNs stated interest in web-based support to improve their smoking cessation counseling. Several PNs were already familiar with other web-based programs for primary care professionals and positively evaluated these programs. They expressed interest in being able to "browse through a program and choose only those things that I can personally apply during counseling'.

Regarding compatibility, PNs expected the content to be up-to-date, individually relevant and applicable in practice. Moreover, a program should be easy to use (low complexity) and require little time investment.

'I want to know the latest developments about smoking cessation... and easily retrieve them from a web-based program, without having to search through databases.'

Long questionnaires and extensive texts were perceived as potential disadvantages and availability after working hours as a potential advantage of web-based support. Furthermore, PNs wanted a program free to visit and re-visit at their convenience without obligations (high trialability). Visually attractive content (i.e. images or videos) and experiences of other PNs were preferred over a text-only form of support (relative advantage).

'Experiences of others would be interesting... []... so you know what was successful and which strategies did not work.'

Most PNs also expressed a need for printable patient-centered educational materials to use during counseling, such as an overview of their patients' counseling trajectory or a timeline depicting physical advantages of quitting smoking.

'The information should be suitable for patients... simple and clear, without complicated terminology. Preferably a combination of text and figures.' 


\section{Discussion and conclusion}

Our results add a novel perspective to the scientific literature on health communication by not only describing PNs' smoking cessation counseling practices, but also determinants of their adherence to evidence-based guidelines as well as their needs regarding a web-based adherence support program. Results indicate that while all PNs reported the use of an evidence-based guideline, their guideline adherence was sub-optimal, which was influenced by psychological and practical barriers. Most PNs showed interest in webbased adherence support that could help them to overcome these barriers and reported specific needs that could be addressed to ensure a program's optimal compatibility to their work situation.

\section{Discussion}

Despite specific difficulties that PNs perceive regarding full adherence to smoking cessation guidelines, they also reported to be confident that they could adapt their counseling to the situation of smoking patients, to stay close to a patient's preferences and beliefs with respect to a quit attempt. In other words, PNs felt able to provide empathic counseling in a collaborative way, which are core elements of motivational interviewing (Copeland, McNamara, Kelson, \& Simpson, 2015), and hence an important competence to possess and apply during smoking cessation consultations.

Nevertheless, PNs also reported psychological and practical barriers regarding their adherence to evidence-based guidelines. A first psychological barrier to guideline adherence was PNs' perceived inability to motivate patients to quit smoking; they reported to rather provide awareness-enhancing information than to actively try to increase patients' motivation, a finding also described in studies investigating nurses' adherence to motivational interviewing techniques (Efraimsson, Fossum, Ehrenberg, Larsson, \& Klang, 2012; Noordman et al., 2012). PNs' perceived inability to motivate smokers could indicate a lack of self-efficacy, which was also found in other studies investigating determinants of nurses' use of guidelines or protocols (Bolman et al., 2002; Cassista, Payne-Gagnon, Martel, \& Gagnon, 2014). Low self-efficacy could possibly originate from PNs' perceptions that they are of minor influence on patients' intrinsic motivation (i.e. low confidence that counseling will make a difference). Relatedly, PNs indicated that they often did not actively try to remove barriers when dealing with unmotivated patients. However, patients' lack of intrinsic motivation could be the result of seeing many barriers for quitting and hence not discussing them could be a missed opportunity to increase patients' motivation to quit (Bundy, 2004). Furthermore, PNs reported difficulties in ensuring their patients' presence at follow-up appointments. This potentially originates from their perception that it is their patients' responsibility to show up. Consequently, they do not repeatedly contact their patients about missed appointments, as was also found in a previous study 
among primary care professionals' smoking cessation counseling practices (van Rossem et al., 2015).

Concerning practical barriers for guideline adherence, PNs perceived a lack of highquality smoking cessation training classes and reported a lack of uniformity about health insurances' compensation policies. Accordingly, PNs had difficulties providing patients with personally relevant and up-to-date information. Additionally, retrieving information about such topics was considered to be very time-consuming, though necessary to enable patients to make an informed choice about their quit attempt. Such time-consuming tasks may cause time constraints regarding actual cessation counseling activities, a commonly mentioned barrier among primary care professionals (Berndt et al., 2013; Studts et al., 2010), and consequently interfere with PNs' adherence to smoking cessation guidelines.

When considering solutions for the identified barriers, most PNs were interested in web-based adherence support. To overcome these barriers PNs expressed the need for tailored support (i.e. instead of offering generic information), compatible with their situation and with low complexity and high trialability. PNs' perceived accessibility after office hours, as a potential advantage of the web-based nature of such a program. These findings are in line with previous studies that related innovation characteristics to increased adoption and implementation of novel interventions by nurses (Smit, de Vries, et al., 2013; van der Weide \& Smits, 2004). Furthermore, PNs would like to be supported with practical tools (e.g. printable patient materials) to apply during counseling and hence improve effective communication about smoking cessation.

\section{Practice Implications}

The development of web-based adherence support seems suitable to help PNs to overcome psychological and practical barriers to improve their smoking cessation guideline adherence. That is, a web-based CT program could provide PNs with personal feedback about performing specific counseling elements they find difficult to apply, based on evidence-based methodologies to change socio-cognitive determinants as described elsewhere (de Ruijter, Smit, de Vries, \& Hoving, 2016). For instance, role modeling could be used to increase self-efficacy, which can be operationalized by presenting PNs with narratives of peers (i.e. fellow PNs) via a web-based program (Kok, Schaalma, Ruiter, van Empelen, \& Brug, 2004). Targeting socio-cognitive determinants through a tailored webbased support program is a well-established method to successfully influence these determinants and ultimately behavior change (Kreuter \& Wray, 2003; Lustria, Cortese, Noar, \& Glueckauf, 2009).

Furthermore, tailored feedback can be supplemented with up-to-date information about smoking cessation counseling in primary care, like an overview of available training classes or health insurances' compensation policies. This could reduce PNs' time needed to find this information and consequently increase their time spent on counseling. Finally, 
PNs can be supported with practical tools, like visually-attractive educational materials for lower educated patients and patients with different cultural backgrounds. Such strategies potentially enable PNs to optimally adhere to evidence-based guidelines and subsequently promote the quality of their smoking cessation care.

\section{Strengths and limitations}

A strength of our study was that the interview guide was based on both the I-Change Model (de Vries et al., 2003) and the Diffusion of Innovations Theory (Rogers, 2003). This enabled us to explore determinants of smoking cessation guideline adherence from both the users' (i.e. PNs) and the innovation's (i.e. web-based adherence support) perspective, a combination which has previously been associated with nurses' adoption and implementation behavior of intervention programs (Segaar, Willemsen, et al., 2007; Smit, de Vries, et al., 2013). Secondly, due to the semi-structured nature of the interview guide PNs had ample opportunity to elaborate on their experiences during smoking cessation counseling, providing a vast amount of data per interview. Moreover, the interviews enabled us to assess PNs' individual preferences for web-based adherence support to optimize such a program's compatibility (Avis, van Mierlo, Fournier, \& Ball, 2015). Thirdly, qualitative data were systematically analyzed using the Framework Method, ensuring optimal coverage of relevant content through the development of an extensive coding tree (Gale et al., 2013). Moreover, intercoder reliability was high, indicating the coding was sufficiently objective and valid.

A limitation of the study was that PNs interested in smoking cessation research may have been more likely to participate in the interviews than non-interested PNs. This may have threatened generalizability of the results to the total population of PNs. Nonetheless, by purposeful sampling we recruited a heterogenic group of PNs who provided a great variability of responses. Moreover, data saturation occurred within the 19 interviews conducted.

\section{Conclusion}

Dutch PNs experienced several barriers to complete smoking cessation guideline adherence. They reported low self-efficacy to motivate patients and to remove patients' barriers to quit, and struggled with successfully organizing patient follow-up. Moreover, a perceived lack of smoking cessation training classes and uniform insurance policies were identified as practical barriers for optimal guideline adherence. PNs were interested in web-based adherence support to overcome these barriers, especially if a web-based program is easy to use, free to (re-)visit and compatible with their current smoking cessation practices. Taking PNs' needs into account when developing a program is likely to stimulate the program's usage and maximize exposure to its content, making it more likely to be effective in improving PNs' smoking cessation guideline adherence. 


\section{Appendix 1: Semi-structured interview guide}

Personal and practice characteristics

What is your age?

Do you currently smoke or have you been a smoker in the past?

What education did you complete in order to work as a practice nurse?

How many years of working experience do you have as a practice nurse?

At how many practices are you employed as a practice nurse?

In-depth question (if applicable): how many hours do you work in these practices?

What is the size of the practice(s), in terms of patients and employees?

Prompt questions

How many patients visit the practice every year?

How many primary care professionals are employed at the practice?

Would you classify the practice(s) as rural or urban?

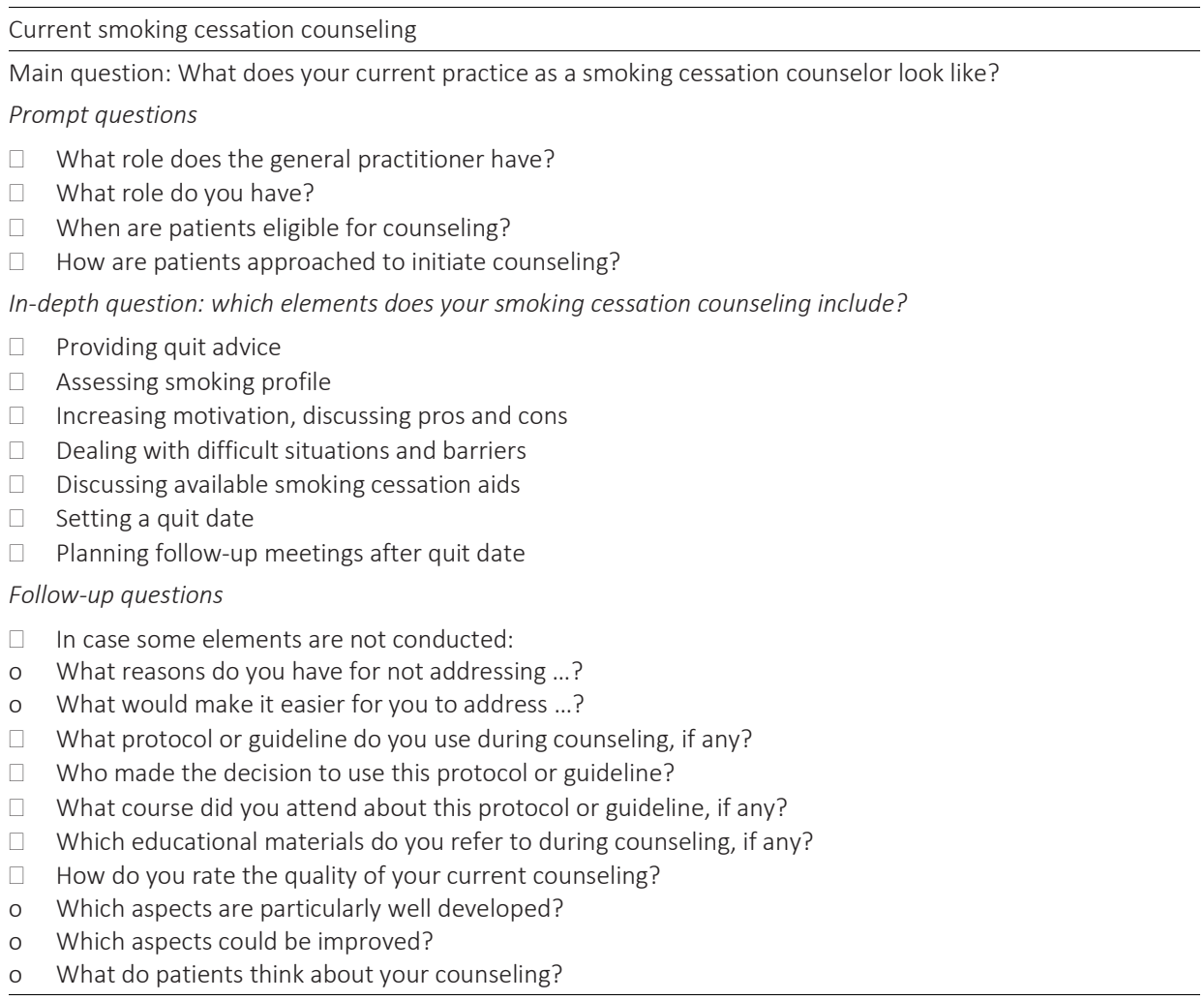

STIMEDIC ${ }^{\circledR}$ smoking cessation guideline*

Main question: What is your opinion of the STIMEDIC ${ }^{\circledR}$ guideline for counseling?

Prompt questions

$\square \quad$ What elements do you find particularly well-developed?

$\square \quad$ Which elements could be difficult to apply in practice?

$\square \quad$ How do you deal with difficult situations in practice?

$\square \quad$ What would be reasons to use the STIMEDIC ${ }^{\circledR}$ guideline? Why not? 
Web-based CT support program**

Main questions:

Why would you (not) be interested in a tailored web-based support program to help you to improve smoking cessation counseling?

What should the content and design of this program look like so you would make use of it?

Prompt questions

$\square \quad$ How much time are you willing to spend on using the program?

$\square \quad$ In what format would you like to receive your tailored advice?

$\square \quad$ What design features should the support program have?

$\square \quad$ What type of content should the advice contain?

$\square \quad$ In case an online forum is available, what possibilities should this provide for you?

$\square \quad$ In case accreditation points are rewarded for using the program, would this affect your use of the program?

$\square \quad$ What other materials or forms of support would you need to improve your smoking cessation counseling?

* Prior to the questions about the STIMEDIC ${ }^{\circledR}$ guideline, PNs were informed about the different steps of the guideline

** Prior to the questions about the web-based CT support program, PNs were informed about the functionalities and relevance of such a program 
Chapter

Dutch practice nurses' adherence to evidence-based smoking cessation treatment guidelines 


\section{Abstract}

Background: Practice nurses in general practice sub-optimally adhere to evidence-based smoking cessation treatment guidelines, but factors explaining their adherence have not yet been investigated. Understanding such factors is important to develop interventions for improving practice nurses' smoking cessation guideline adherence and patients' subsequent cessation success. This study explored the association between different sociocognitive and predisposing factors, and practice nurses' adherence to the Dutch smoking cessation guideline in general (i.e. overall adherence) and to each guideline step individually (i.e. step-based adherence).

Methods: A cross-sectional study was conducted among practice nurses ( $N=157)$ in January-March 2015 via web-based questionnaires, assessing constructs from the Integrated Change Model. Spearman's correlations and linear regression analysis were used to identify potential determinants of overall guideline adherence; Mann-Whitney U-tests and logistic regression analyses were used to identify potential determinants of step-based adherence.

Results: On average five out of nine steps were completely adhered to by practice nurses; and step-based adherence ranged from 34-75\%. Overall guideline adherence was associated with high levels of self-efficacy to use a guideline $(\beta=.32, p=.00)$, and step-based adherence was additionally associated with spending more time on counseling. Regression results showed positive associations between self-efficacy (8/9 steps) and perceived advantages (7/9 steps) with step-based adherence.

Conclusion: This study quantitatively confirmed practice nurses' sub-optimal guideline adherence and found associations between socio-cognitive (self-efficacy and perceived advantages) and predisposing factors (time spent on counseling), and guideline adherence. Detailed insights in these factors offer preliminary directions for intervention development to improve practice nurses' adherence to evidence-based smoking cessation guidelines. 


\section{Background}

Smoking is still the leading cause of preventable disease and premature death worldwide (WHO, 2015). In the Netherlands, about 23\% of the adult population still smokes (RIVM, 2014), despite effective smoking cessation strategies like a brief advice by a general practitioner (GP), ideally followed by more intensive forms of counseling (Stead et al., 2013). In Dutch general practices (family practice) such smoking cessation support is increasingly provided by trained practice nurses (PNs) (Freund et al., 2015); highly educated nurses who are mainly responsible for chronic patient care (Heiligers et al., 2012) and are now employed in over $80 \%$ of Dutch general practices (Freund et al., 2015).

To structure their smoking cessation counseling sessions, PNs are trained to use national evidence-based smoking cessation guidelines, of which the STIMEDIC ${ }^{\circledR}$ stop smoking guideline is the most recent and up-to-date (Stop Smoking Partnership, 2009). This guideline describes a step-wise approach of smoking cessation support combining behavioral counseling, with pharmacological support (Stop Smoking Partnership, 2009). Full adherence to such guidelines by health professionals positively contributes to quality of care (Miller \& Kearney, 2004) and has stronger beneficial effects on patients' quit rates than a brief quit advice (Rice et al., 2013). Nevertheless, previous research has established nurses' sub-optimal adherence to smoking cessation guidelines (Berndt et al., 2013; Segaar, Willemsen, et al., 2007).

Various socio-cognitive and predisposing factors can influence PNs' adherence to these guidelines, but research into these factors is scarce. So far, only recent qualitative studies have investigated this subject, establishing that factors, such as low self-efficacy to enhance a smoker's motivation (de Ruijter, Smit, de Vries, Goossens, \& Hoving, 2017) and the belief that most smokers are unwilling to quit, might play an important role in explaining PNs' guideline adherence (van Rossem et al., 2015).

Although these studies provide some insights into the factors associated with guideline adherence, obtaining quantitative evidence about the supposed associations between these factors and guideline adherence is needed to inform guideline adherence intervention development. Additionally, no studies so far have focused on factors associated with adherence to individual guideline steps (i.e. step-based adherence), even though a previous study showed some steps are better adhered to than others (van Rossem et al., 2015). Moreover, since predominantly attitude and self-efficacy might play an important role in explaining PNs' smoking cessation guideline adherence (de Ruijter, Smit, de Vries, Goossens, et al., 2017), the association between these socio-cognitive concepts and step-based adherence is especially interesting to investigate.

Therefore, the aim of the present study was to determine the association between socio-cognitive and predisposing factors, and PNs' overall and step-based adherence to evidence-based smoking cessation guidelines in the Netherlands. 


\section{Methods}

A cross-sectional questionnaire study was conducted among Dutch PNs. Online questionnaire content was based on the Integrated Change (I-Change) Model (de Vries et al., 2003) and an earlier qualitative study (de Ruijter, Smit, de Vries, Goossens, et al., 2017).

\section{The Integrated Change Model}

The I-Change Model is an integrated model for explaining motivational and behavioral change (de Vries et al., 2003). Previous studies based on the I-Change Model have demonstrated its usefulness in explaining health professionals' behaviors related to smoking cessation (Berndt et al., 2013; Smit, de Vries, et al., 2013). The I-Change Model describes that the intention to perform a behavior is most proximally determined by the motivational constructs (i.e. socio-cognitive factors) attitude (perceived (dis)advantages), perceived social influence (norms, modeling and pressure/support) and self-efficacy. The model also includes predisposing factors (e.g. PNs' level of training) which may directly influence behavior, or indirectly influence behavior through the socio-cognitive factors (de Vries et al., 2003).

\section{Participants and procedure}

Eligible participants were employed as a PN or specialized nurse (e.g. specialization in diabetes or lung problems) in a Dutch general practice, actively engaged in smoking cessation counseling and had Internet access. Interested PNs received information on data protection and anonymity, and all participants provided informed consent prior to completing the questionnaire, participation was voluntary. Completion of the online questionnaire took about 20 minutes. Participants who completely filled out the questionnaire could opt to provide their email address to enter a raffle to win one of two $€ 50$ gift vouchers.

PNs were recruited between January-March 2015 via e-mails to networks of PNs and nurses; PN or nurse interest group newsletters; and recruitment messages on relevant Social Media pages (e.g. LinkedIn, Facebook). Recruitment materials included a hyperlink to the online questionnaire. Alternatively, PNs could visit the project website www.sterstudie.nl (Self-Archived at WebCite ${ }^{\circledR}$ on 26 July 2016 [http://www.webcitation.org/6jHiDBycb]) for more information and gain access to the online questionnaire there.

\section{Measurements}

The online questionnaire included questions about demographics, predisposing factors, smoking cessation guideline adherence, and socio-cognitive factors (attitude, self-efficacy and perceived social influence). 
Demographics. Age, gender and smoking status (smoker, ex-smoker, non-smoker) were assessed.

Predisposing factors. Work experience as a nurse in general and as a smoking cessation counselor was assessed in years. Furthermore, participants were asked whether they had attended a course on smoking cessation counseling during the last year $(\mathrm{y} / \mathrm{n})$ and whether they were registered $(y / n)$ in the Dutch Stop Smoking quality register (www.kwaliteitsregisterstopmetroken.nl, Self-Archived at WebCite ${ }^{\circledR}$ on 26 July 2016 [http://www.webcitation.org/6jHxBZKfn]). Also, the average number of smoking patients counseled per year, average number of consultations per counseled smoker and the duration of an average smoking cessation consultation in minutes were assessed, which were subsequently combined to represent the total time a participant spent on smoking cessation counseling per year. Participants were also asked about percentage of smokers who's smoking cessation counseling would be reimbursed by their health insurance ( $1=$ nobody, $5=$ everyone) and in how many practices they were employed ( $1=$ one practice; $2=$ more than one practice). As part-time employment is common for PNs (Heiligers et al., 2012), participants were instructed to answer the questions for the general practice in which they worked most hours per week. Questions concerned the estimated number of registered patients in the practice, the presence of special smoking cessation counseling hours $(\mathrm{y} / \mathrm{n})$ and whether patients' smoking status was systematically registered in their patient file $(y / n)$. Finally, participants were asked about their use of an evidencebased smoking cessation guideline during consultations ( $y / n)$; if yes, also for the time period in which they had been using that guideline (in years) and whether they used a visual overview of that guideline during counseling $(\mathrm{y} / \mathrm{n})$.

Guideline adherence. Questions concerned the nine counseling steps of the STIMEDIC ${ }^{\circledR}$ guideline (Stop Smoking Partnership, 2009): 1) offering a quit advice, 2) assessing smoking profile and smoking history, 3) assessing motivation to quit, 4) increasing motivation, 5) assessing barriers to quitting, 6) discussing barriers, 7) informing about cessation aids, 8) making a quit plan and setting a quit date, and 9) arranging follow-up after the quit date. Step-based guideline adherence was assessed by asking how often participants performed each step during smoking cessation counseling sessions ( $1=$ never, $4=$ always). Data was dichotomized, using the $50^{\text {th }}$ percentile as cut-off score, to create a 'not adherent' group (scores 1-3) and an 'adherent' group (score 4). To measure overall guideline adherence, an index score was created by adding the dichotomized scores, resulting in an overall adherence score ranging from 0-9.

Socio-cognitive factors. For each smoking cessation counseling step, participants responded to statements regarding attitude (perceived advantages and disadvantages) and their self-efficacy to apply this step in practice (1=completely disagree, $5=$ completely agree). For example, concerning the step offering a quit advice the following statements were presented: if I offer a quit advice, my patients are more likely to make a quit attempt 
(perceived advantage); if I offer a quit advice, this results in negative reactions of my patients (perceived disadvantage) and I think I am able to offer a quit advice to my patients (self-efficacy).

Subsequent questions about perceived social influence addressed participants' overall adherence to evidence-based guidelines (1=completely disagree, 3=neutral/not applicable, 5=completely agree). Participants responded to five items about the degree to which important others (their GP, PNs working in the same practice or in other practices, the practice manager and practice patients) considered using a smoking cessation guideline as important (social norm) and supported them (social support) to use such a guideline. Finally, participants responded to three items about whether their GP and other PNs (within the same practice and in other practices) make use of such guidelines while counseling smokers (modelling).

\section{Data analysis}

Analyses were conducted using SPSS version 21.0. To investigate the association between socio-cognitive factors and guideline adherence, scales were created. Reliability analyses showed sufficient internal consistency for perceived advantages $(\alpha=.78)$, perceived disadvantages ( $\alpha=.77)$, self-efficacy ( $\alpha=.75)$, social norms ( $\alpha=.82)$ and social support $(\alpha=.70)$ scales. The three modeling items were included separately in analyses.

Several non-parametric analyses were performed, as normality of main variables was not established. To investigate associations between socio-cognitive and predisposing factors, and PNs' overall guideline adherence, Spearman's rank correlation coefficients were calculated. Predisposing factors (using a visual guideline overview, recently completing a smoking cessation training, the availability of special counseling hours, counseling experience and time spent on counseling) were selected for inclusion in these analyses based on earlier research findings (Smit, de Vries, et al., 2013; van der Weide \& Smits, 2004). Next, hierarchical backward linear regression analysis was conducted to assess which socio-cognitive factors could explain variance in overall guideline adherence, correcting for predisposing factors that significantly correlated with overall guideline adherence.

Differences between adherers and non-adherers in terms of their step-based adherence were assessed using Mann-Whitney $U$ tests. Moreover, hierarchical backward logistic regression analyses were conducted to assess which socio-cognitive factors could explain variance in adherence to each guideline step, correcting for the same predisposing factors as in the linear regression analysis. 


\section{Results}

\section{Sample characteristics}

Characteristics of participating PNs ( $\mathrm{N}=157$, response rate $70.1 \%)$ are shown in Table 1. Estimations of the number of registered patients at their general practice showed great variation, though was on average rather high compared to a Dutch standard size practice $(2,168$ patients) per fulltime GP (Batenburg, 2016). This may indicate that most respondents were employed within a group practice. The reported amount of time spent on cessation counseling also showed a lot of variation, ranging from 2.1 to 264 hours per year. Overall, PNs reported to completely adhere to five out of nine steps. The highest percentage of PNs (75\%) fully adhered to assessing motivation to quit, whereas only $40 \%$ and $34 \%$ of PNs reported to fully adhere to increasing motivation and discussing barriers respectively.

Table 1 Characteristics of Dutch practice nurses ( $N=157)$, recruited between January-March 2015

\begin{tabular}{|c|c|c|}
\hline Characteristics & $\%$ & Mean (SD) \\
\hline Female gender & 98.0 & \\
\hline Age & & $47.3(9.8)$ \\
\hline $\begin{array}{l}\text { Smoking status: } \\
\text { non-smoker } \\
\text { ex-smoker }\end{array}$ & $\begin{array}{l}55.4 \\
44.1\end{array}$ & \\
\hline Employed in 1 practice & 64.5 & \\
\hline Number of registered patients in practice & & $4647(2864)$ \\
\hline Using evidence-based guidelines & 86.5 & \\
\hline Recently followed smoking cessation course & 67.3 & \\
\hline Experience in using a guideline in years & & $4.6(2.6)$ \\
\hline Using visual guideline overview & 54.7 & \\
\hline Special smoking cessation hours in practice & 50.0 & \\
\hline Experience in smoking cessation counseling in years & & $5.6(3.1)$ \\
\hline Patients' smoking status systematically registered & 100.0 & \\
\hline Reimbursement for smoking cessation counseling ${ }^{a}$ & & $4.1(1.3)$ \\
\hline Registered in quality register for smoking cessation & 72.8 & \\
\hline Time spent per year on counseling smokers in hours & & $45.9(44.8)$ \\
\hline Overall adherence score $(\mathrm{Md})^{\mathrm{b}}$ & & 5 \\
\hline
\end{tabular}

Note: a range from $1-5 ;{ }^{b}$ range from $0-9$

\section{Overall guideline adherence and potential determinants}

PNs' overall guideline adherence was found to significantly and positively correlate with perceived advantages ( $\left.r_{s}=.36 ; p=.00\right)$ of guideline use and PNs' self-efficacy $\left(r_{s}=.40 ; p=.00\right)$ to use such a guideline (Appendix 1). Furthermore, the amount of time spent on counsel- 
ing was found to significantly correlate with guideline adherence, indicating that adherent PNs spent more time on counseling per year. Results from the backward linear regression analysis showed however that only self-efficacy $(\beta=.32, p=.00)$ significantly contributed to explaining PNs' overall guideline adherence.

\section{Step-based adherence and potential determinants}

Results from Mann-Whitney U-tests show that higher self-efficacy and more perceived advantages were significantly associated with complete adherence to all guideline steps, except for offering a quit advice and assessing motivation to quit (Appendix 2). Results from backward logistic regression analyses, showing only factors contributing to the model, indicate that higher self-efficacy and spending more time on counseling were found to be significantly associated with better adherence to six and five guideline steps, respectively (Table 2). Moreover, more perceived advantages was significantly associated with better adherence to discussing barriers $(O R=2.09)$ and more perceived disadvantages to informing about cessation aids $(\mathrm{OR}=1.87)$.

\section{Discussion of results}

The present study investigated the extent of PNs' smoking cessation treatment guideline adherence and explored associations between their adherence and its potential determinants. Results illustrated Dutch PNs' sub-optimal adherence to evidence-based smoking cessation guidelines; overall PNs reported to adhere to five of nine guideline steps and their step-based adherence ranged from 34\% to 75\%. Self-efficacy and time spent on counseling were important factors explaining variation in PNs' overall and step-based adherence. Moreover, perceived advantages of guideline use were positively associated with PNs' step-based adherence fairly consistently.

PNs' self-reported adherence was lowest for guideline steps increasing motivation (40\%) and discussing barriers (34\%). These findings corroborate results from earlier qualitative studies, in which PNs describe often skipping these steps when smokers are perceived to be not motivated enough to quit (de Ruijter, Smit, de Vries, Goossens, et al., 2017) or when they perceive it as smokers' own responsibility to become motivated to quit smoking (van Rossem et al., 2015). This could also explain why $75 \%$ of PNs in the present study completely adhered to assessing motivation to quit, but only $34 \%$ to $55 \%$ reported to adhere to the next five guideline steps. As these five steps address key elements (i.e. behavioral and pharmacological support) from evidence-based smoking cessation guidelines that have shown to significantly improve cessation success in smokers (Miller \& Kearney, 2004; Rice et al., 2013), it could hence be worthwhile to especially target these elements in adherence-promoting interventions or trainings for PNs. 
Table 2 Results of hierarchical logistic regression analyses with potential determinants of practice nurses' stepbased adherence $(n=148)$.

\begin{tabular}{|c|c|c|c|}
\hline & OR & $\begin{array}{l}95 \% \mathrm{Cl} \\
\text { Lower }\end{array}$ & Upper \\
\hline \multicolumn{4}{|l|}{ Offering a quit advice } \\
\hline Time spent on counseling & 1.00 & .99 & 1.01 \\
\hline Self-efficacy & $2.75^{* *}$ & 1.52 & 4.98 \\
\hline \multicolumn{4}{|c|}{ Assessing smoking profile and smoking history } \\
\hline Time spent on counseling & 1.00 & .99 & 1.01 \\
\hline Self-efficacy & $2.13^{*}$ & 1.11 & 4.09 \\
\hline \multicolumn{4}{|l|}{ Assessing motivation to quit } \\
\hline Time spent on counseling & 1.01 & .99 & 1.03 \\
\hline \multicolumn{4}{|l|}{ Increasing motivation } \\
\hline Time spent on counseling & $1.02 * *$ & 1.01 & 1.03 \\
\hline Self-efficacy & $2.68^{* *}$ & 1.39 & 5.15 \\
\hline \multicolumn{4}{|l|}{ Assessing barriers to quitting } \\
\hline Time spent on counseling & $1.02 * *$ & 1.01 & 1.04 \\
\hline \multicolumn{4}{|l|}{ Discussing barriers } \\
\hline Time spent on counseling & $1.02 * *$ & 1.01 & 1.03 \\
\hline Self-efficacy & 1.89 & .96 & 3.70 \\
\hline Perceived advantage & $2.09 *$ & 1.04 & 4.23 \\
\hline \multicolumn{4}{|l|}{ Informing about cessation aids } \\
\hline Time spent on counseling & $1.01 *$ & 1.00 & 1.02 \\
\hline Self-efficacy & $2.87 * *$ & 1.43 & 5.76 \\
\hline Perceived disadvantage & $1.87^{*}$ & 1.07 & 3.27 \\
\hline \multicolumn{4}{|c|}{ Making a quit plan and setting a quit date } \\
\hline Time spent on counseling & $1.01 *$ & 1.00 & 1.02 \\
\hline Self-efficacy & $1.99 *$ & 1.08 & 3.66 \\
\hline Modeling PNs in other practices & .64 & .40 & 1.01 \\
\hline \multicolumn{4}{|l|}{ Arranging follow-up after the quit date } \\
\hline Time spent on counseling & 1.00 & .99 & .101 \\
\hline Self-efficacy & $2.54^{* *}$ & 1.34 & 4.79 \\
\hline
\end{tabular}

Note: ${ }^{* *} p<.01 * p<.05$

Spending more time on counseling, perceiving more advantages and having high self-efficacy were associated with better overall adherence by PNs, which matches findings from previous research identifying barriers to guideline adherence among nurses (Abrahamson, Fox, \& Doebbeling, 2012). Yet, a PN spending a lot of time on counseling could either counsel many patients, or spend more time per patient. Our data shows that adherent PNs counseled approximately twice as many patients than non-adherent PNs, whereas the time spent per consultation was similar for all PNs (data not shown). Thus, adherent PNs seem more engaged in smoking cessation counseling, which may not only increase their efficiency in applying the guideline, but also increase their self-efficacy to use such a guideline. Although our results indeed showed that PNs' self-efficacy was positively correlated with time spent on counseling, we cannot determine causality from the present data. 
In contrast to previous research findings (Berndt et al., 2013; Segaar, Willemsen, et al., 2007), no associations between PNs' overall adherence and perceived social influence and perceived disadvantages were found. This may be explained by all PNs in our study scoring rather neutral regarding perceived social influence and somewhat low on perceived disadvantages of applying a guideline. Whereas the latter suggest that there might not be much room for improvement, neutral results regarding perceived social influence might indicate that PNs' social environment is not very important when it comes to their guideline adherence. Though, many PNs indicated that no practice manager (up to 65\%) or other PNs (up to 46\%) were employed in their practice, probably pushing mean scores on perceived social influence towards a more neutral mean. Not considering these neutral scores, PNs still reported mostly neutral perceived social influence (social norms 3.29, social support 3.14, social modeling 3.58; on a five-point scale) from their GP, patients and PNs in other practices regarding their use of an evidence-based guideline. As previous studies did find associations of social influence with intention to use a smoking cessation protocol by health care professionals (Berndt et al., 2013; Smit, de Vries, et al., 2013), in future studies it could be worthwhile to examine how such associations can also be established for PNs to improve their smoking cessation guideline adherence.

Regarding step-based adherence, self-efficacy and perceived advantages were found to be associated with PNs' complete adherence to most guideline steps, corresponding with previous findings among nurses (Segaar, Willemsen, et al., 2007). Especially PNs' level of self-efficacy appears to play an important role as it significantly explained variance in adherence to eight of the nine guideline steps. It would hence be key to further investigate this, for instance by assessing PNs' step-based self-efficacy in a range of situations where it might prove more difficult to adhere to these guideline steps, such as when they are behind schedule or when they experience rejection from smokers (Smit, Hoving, Schelleman-Offermans, West, \& de Vries, 2014). This could produce detailed information on circumstances and situations that are associated with PNs' (non-)adherence to individual guideline steps and provide specific directions for intervention.

Finally, results concerning the guideline step informing about cessation aids showed that adherent PNs felt more strongly about the disadvantage (i.e. patients believe that merely using a cessation aid is sufficient to quit smoking) associated with performing this counseling step. Though only found for a single step, this finding is not in line with our theoretical framework (de Vries et al., 2003) and previous research (Berndt et al., 2013; Segaar, Willemsen, et al., 2007). However, as adherent PNs apply this particular guideline step more often in practice, they could also more often encounter patients believing that merely cessation aids are sufficient to quit smoking, but not perceiving this as a reason to stop applying guideline-based cessation counseling on a regular basis. 


\section{Practice implications}

In general, our results showed that similar socio-cognitive (perceived advantages and self-efficacy) and predisposing factors (time spent on counseling) were associated with both PNs' overall and step-based adherence. These associations combined with detailed results regarding PNs' step-based adherence, provide good foci to improve PNs' guideline adherence via interventions or training. Taking PNs' time constraints (van Rossem et al., 2015) and interest in a personally relevant, practically applicable program (de Ruijter, Smit, de Vries, Goossens, et al., 2017) into consideration, an internet-based intervention that is tailored to determinants of PNs' adherence could be a feasible support tool for PNs (Tate et al., 2009). Though research has established the effectiveness of tailored interventions in changing various health behaviors and their determinants (Kohl et al., 2013), so far such interventions for health care professionals to improve their smoking cessation guideline adherence do not yet exist. Therefore, the present study's results can potentially be used as a starting point for developing (tailored) interventions for PNs to support them with adhering to evidence-based smoking cessation guidelines.

\section{Strengths and limitations}

A strength of the present study is that its design was based on the I-Change Model (de Vries et al., 2003), and previous studies investigating the use of smoking cessation interventions among health care professionals (Berndt et al., 2013; Smit, de Vries, et al., 2013). Also, to the best of our knowledge, the present study is the first to operationalize a stepbased approach to guideline adherence and therefore the results provide a more detailed picture of factors associated with PNs' adherence to smoking cessation guidelines.

However, the study's cross-sectional nature prevents us from drawing conclusions about how and to what extent constructs are causally linked. For this purpose, longitudinal follow-up studies are needed. Results are also limited to our self-selected sample, of potentially more motivated participants, recruited from the population of Dutch PNs and some caution is therefore warranted when generalizing results to other professions and health care systems. Nevertheless, the number of complete questionnaires filled out by PNs was found to be quite reasonable (response rate 70.1\%). Finally, PNs might have over-estimated their guideline adherence, as is often observed when collecting self-reported data (Gnambs \& Kaspar, 2016). To account for this, we have considered only those PNs who reported to always perform a guideline step as adherent.

\section{Conclusions}

This study confirmed Dutch PNs' sub-optimal overall (five out of nine steps) and stepbased (34\% to $75 \%$ ) adherence to evidence-based smoking cessation guidelines. Detailed insights in associations between PNs' guideline adherence and socio-cognitive (perceived 
advantages and self-efficacy) and predisposing factors (time spent on counseling), can inform the development of interventions for PNs to improve their overall and step-based adherence to evidence-based smoking cessation guidelines. 


\section{Appendix 1: Spearman's rank correlation matrix}

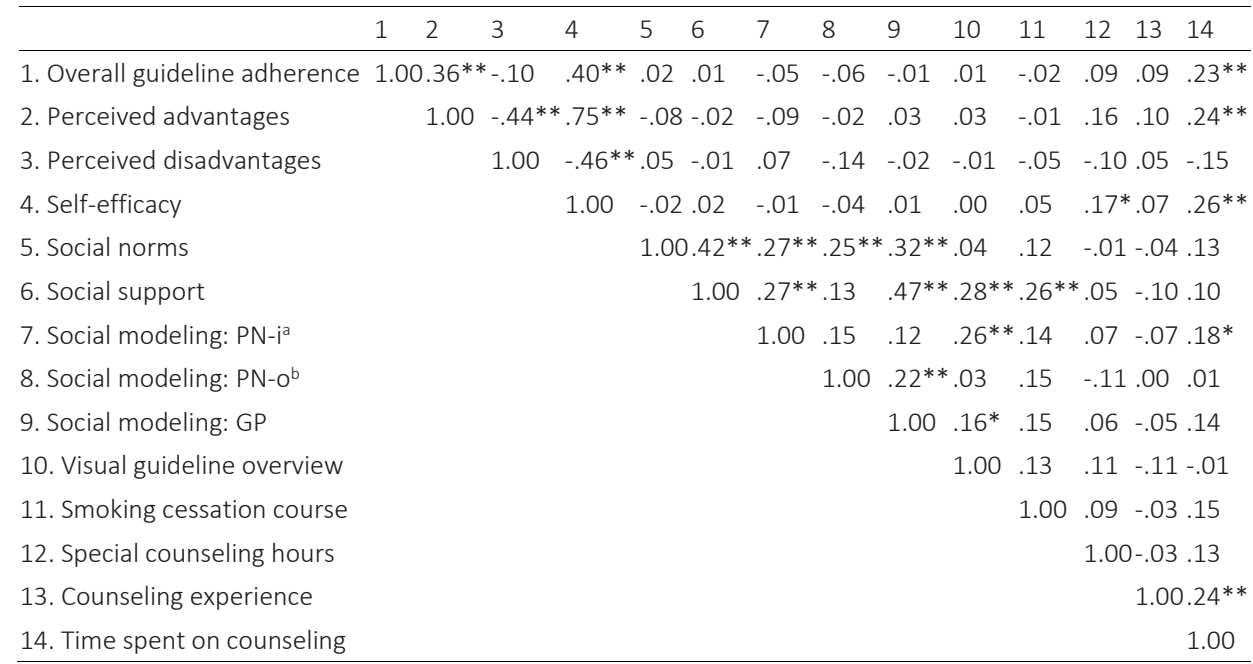

Note: ${ }^{* *} p<.01 * P<.05 ;{ }^{a}$ within the same practice; ${ }^{b}$ in other practices 


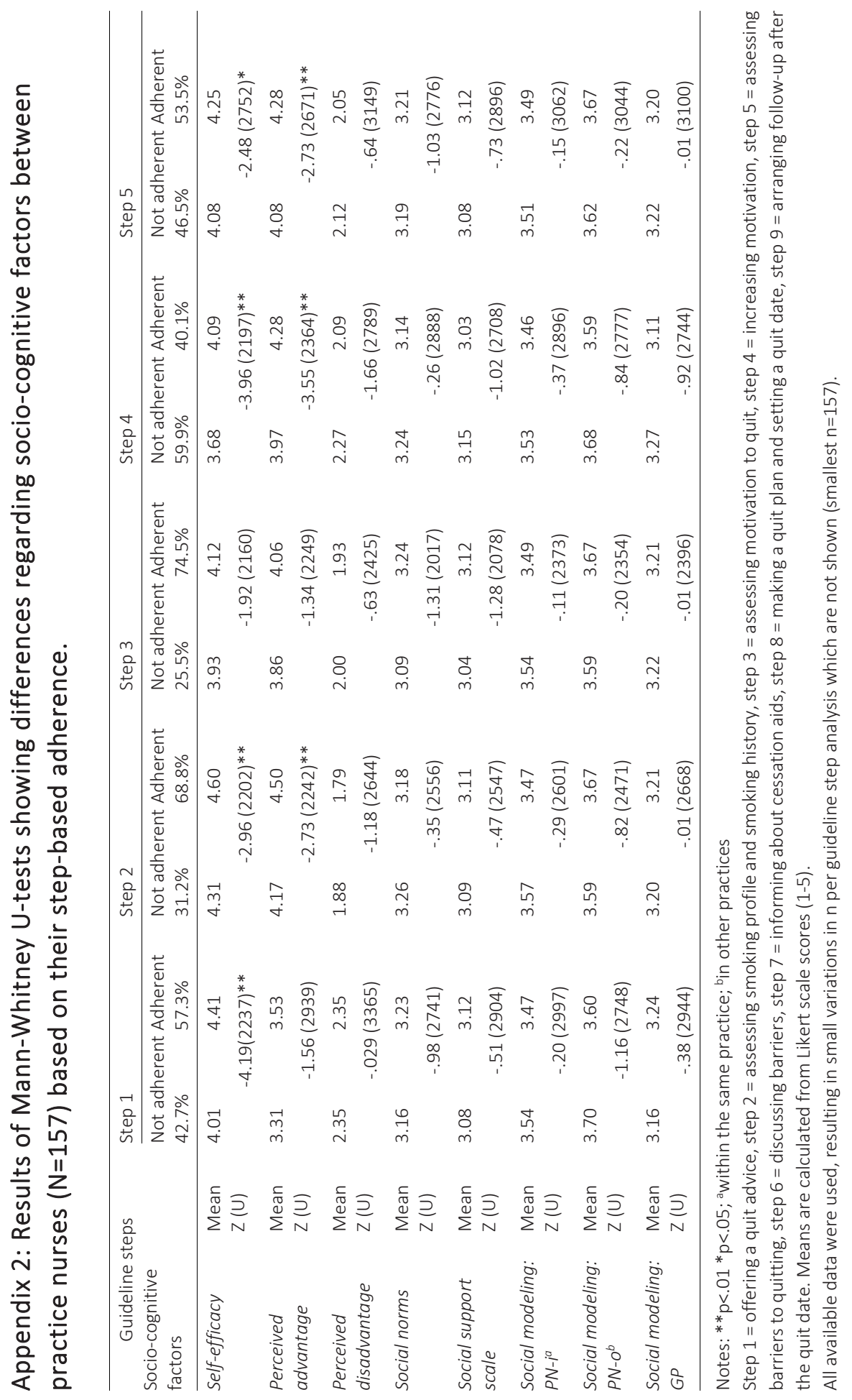




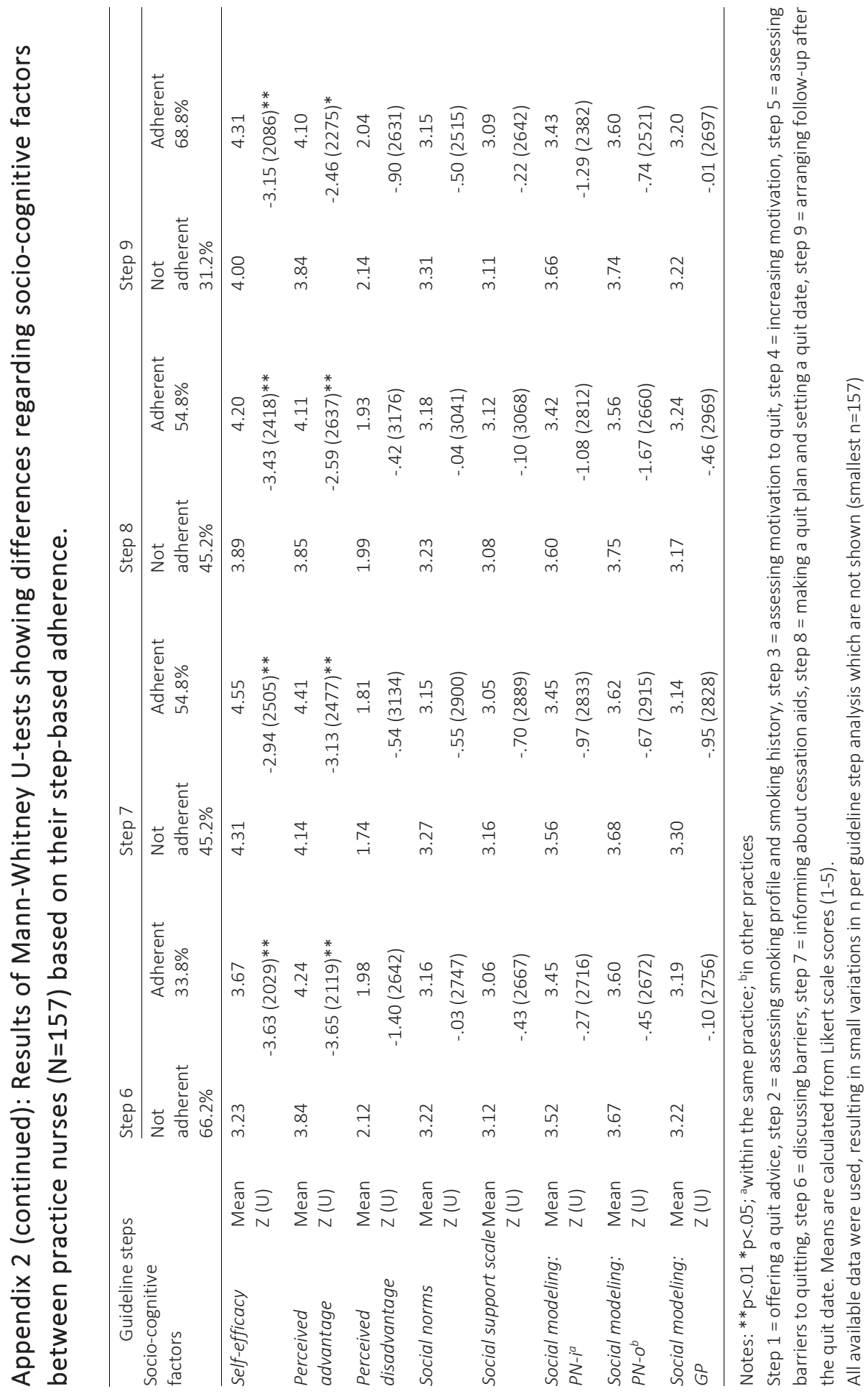



Chapter

Web-based computer-tailoring for practice nurses aimed to improve smoking cessation guideline adherence:

A study protocol for a randomized controlled effectiveness trial. 


\section{Abstract}

Background: Dutch practice nurses sub-optimally adhere to evidence-based smoking cessation guidelines. Web-based computer-tailoring could be effective in improving their guideline adherence. Therefore, this paper aims to describe the development of a webbased computer-tailored program and the design of a randomized controlled trial testing its (cost-)effectiveness.

Methods: Theoretically grounded in the I-Change Model and Self-Determination Theory, and based on the results of a qualitative needs assessment among practice nurses, a webbased computer-tailored program was developed including three modules with tailored advice, an online forum, modules with up-to-date information about smoking cessation, Frequently Asked Questions (FAQs) and project information, and a counseling checklist. The program's effects are assessed by comparing an intervention group (access to all modules) with a control group (access to FAQs, project information and counseling checklist only). Smoking cessation guideline adherence and behavioral predictors (i.e. intention, knowledge, attitude, self-efficacy, social influence, action and coping planning) are measured at baseline and at 6- and 12-month follow-up. Additionally, the program's indirect effects on smokers' quit rates and the number of quit attempts are assessed after 6 and 12 months.

Discussion: This paper describes the development of a web-based computer-tailored adherence support program for practice nurses and the study design of a randomized controlled trial testing its (cost-)effectiveness. This program potentially contributes to improving the quality of smoking cessation care in Dutch general practices. If proven effective, the program could be adapted for use by other healthcare professionals, increasing the public health benefits of improved smoking cessation counseling for smokers. 


\section{Introduction}

Smoking continues to be the leading cause of preventable disease and premature death worldwide (USDHHS, 2014; WHO, 2013). In the Netherlands, smoking prevalence has been decreasing for years, but about $23 \%$ of the adult population still smokes (RIVM, 2014). Evidence-based smoking cessation interventions combining pharmacotherapy with behavioral counseling are effective in supporting smokers to quit (Martin Cantera et al., 2015; Patnode et al., 2015; Stead, Koilpillai, \& Lancaster, 2015). Dutch smokers most often visit their general practice for such cessation support, which is increasingly provided by trained practice nurses (PNs) (Freund et al., 2015; Nederlands Huisartsen Genootschap \& Landelijke Huisarsten Vereniging, 2011). PNs are employed in $80 \%$ of general practices, taking over specific tasks from the GP such as chronic disease consultations and lifestyle counseling, like smoking cessation support (Heiligers et al., 2012).

PNs are qualified to provide smoking cessation counseling (Heiligers et al., 2012), but their adherence to evidence-based smoking cessation guidelines has proven to be suboptimal (i.e. partial adherence) (Glasgow et al., 2003; Segaar, Willemsen, et al., 2007; Whitehead et al., 2014). Complete adherence to these guidelines is known to have better effects on patients' quit rates than only a brief quit advice or less elaborate counseling (Rice et al., 2013). As improved guideline adherence positively contributes to the quality of smoking cessation care (Heiligers et al., 2012; Miller \& Kearney, 2004), it is therefore important to improve PNs' smoking cessation guideline adherence.

Dutch PNs' suboptimal guideline adherence can be explained by various perceived barriers, such as low self-efficacy to motivate smokers and use motivational interviewing techniques; difficulties finding up-to-date information about smoking cessation, compensation of counseling and high-quality training opportunities for PNs; and not considering cessation counseling as their responsibility (de Ruijter, Smit, de Vries, Goossens, et al., 2017; Heiligers et al., 2012; van Rossem et al., 2015). Particularly guideline elements like 'motivating smokers to quit' and 'organizing adequate follow-up consultations' are regularly not adhered to in practice (de Ruijter, Smit, de Vries, Goossens, et al., 2017). Recognizing these barriers to smoking cessation guideline adherence, Dutch PNs have recently indicated interest in a tailored, easy-to-use, web-based program that can provide them with guideline adherence support (de Ruijter, Smit, de Vries, Goossens, et al., 2017).

Such guideline adherence support can be provided via web-based computer-tailored (CT) programs, providing PNs with personally relevant advice, based on their individual answers to the questions of an online questionnaire (Kreuter \& Wray, 2003). When PNs, for instance, indicate specific difficulties for adhering to evidence-based smoking cessation guidelines they subsequently receive CT advice about only those difficult situations (e.g. when patients have little time) that they individually perceive as barriers to adherence (de Vries \& Brug, 1999). Integrating CT advice in a web-based environment makes it easily accessible, since PNs can consult it wherever and whenever they find convenient (Griffiths et al., 2006; Tate et al., 2009). 
CT programs have proven to be effective in changing various health behaviors and their determinants (Civljak et al., 2013; Kohl et al., 2013; Lustria et al., 2013). CT programs for smokers, for example, were found to be effective in improving quit rates (Bolman et al., 2015; Elfeddali et al., 2012; Smit et al., 2012; Stanczyk, Bolman, et al., 2014) and a CT intervention for physicians showed promising results regarding adherence to smoking cessation guidelines (Unrod et al., 2007). We therefore hypothesize that CT advice on applying evidence-based smoking cessation guidelines can effectively support PNs to improve their guideline adherence. Ultimately, CT advice could contribute to improving the quality of smoking cessation care in general practices, resulting in more successful quit attempts among counseled smokers and less smoking related illness and death. Nonetheless, a CT program for PNs aimed at improving their smoking cessation guideline adherence does not yet exist.

Therefore, this paper describes the development of a web-based CT adherence support program and its components for Dutch PNs. Additionally, the study design of a randomized controlled trial testing the program's (cost-)effectiveness is described.

\section{Methods/design}

The aim of this paper is to describe the development of a web-based CT adherence support program for PNs and the study design of the randomized controlled trial testing the effectiveness of this program in terms of guideline adherence by the PN and subsequent smoking cessation rates among their smoking patients. Evaluation by the Medical Ethics Committee Atrium-Orbis-Zuyd (14-N-17) revealed that no medical ethical clearance for this study was needed according to the rules of the Medical Research Involving Human Subjects Act (WMO). The study is registered with the Dutch Trial Register (NTR4436).

\section{Study design effectiveness trial}

In order to investigate the effects of the web-based CT program on PNs' adherence to evidence-based smoking cessation guidelines, a randomized controlled trial will be conducted. PNs in the intervention group have full access to the content of the web-based CT program (i.e. five intervention modules and three general modules), while PNs in the control group have access to the three general modules of the web-based CT program only.

At the start of the trial all participating PNs are asked to fill out a baseline questionnaire. Upon completion of this questionnaire, only PNs in the intervention group receive a tailored feedback letter (which PNs can later also consult in one of the intervention modules). Immediately after receiving this feedback letter, intervention group PNs have access to five intervention modules i.e. brief tailored advice, extended tailored advice, new tailored advice, an online forum, and background information) and three general modules (i.e. project information, frequently asked questions (FAQs), and a counseling 
checklist). PNs are free to (re-)visit and use these modules at their convenience during this six-month period.

After completion of the baseline questionnaire, PNs in the control group receive a short thank-you message. During the subsequent six months, these PNs do not have access to the five intervention modules of the web-based CT adherence support program, but only to the three general modules: project information, the FAQs and the counseling checklist. PNs in this group can also (re-)visit and use these modules at their convenience.

Once the six-month intervention period ends and PNs in both the intervention and control group have filled out the first follow-up questionnaire, only project information, the FAQs and the counseling checklist remain accessible for both groups. Intervention group PNs will no longer have access to the five intervention modules.

The three general modules remain available until PNs from both groups receive the invitation to fill out the second follow-up questionnaire, 12 months after baseline (Figure 1).

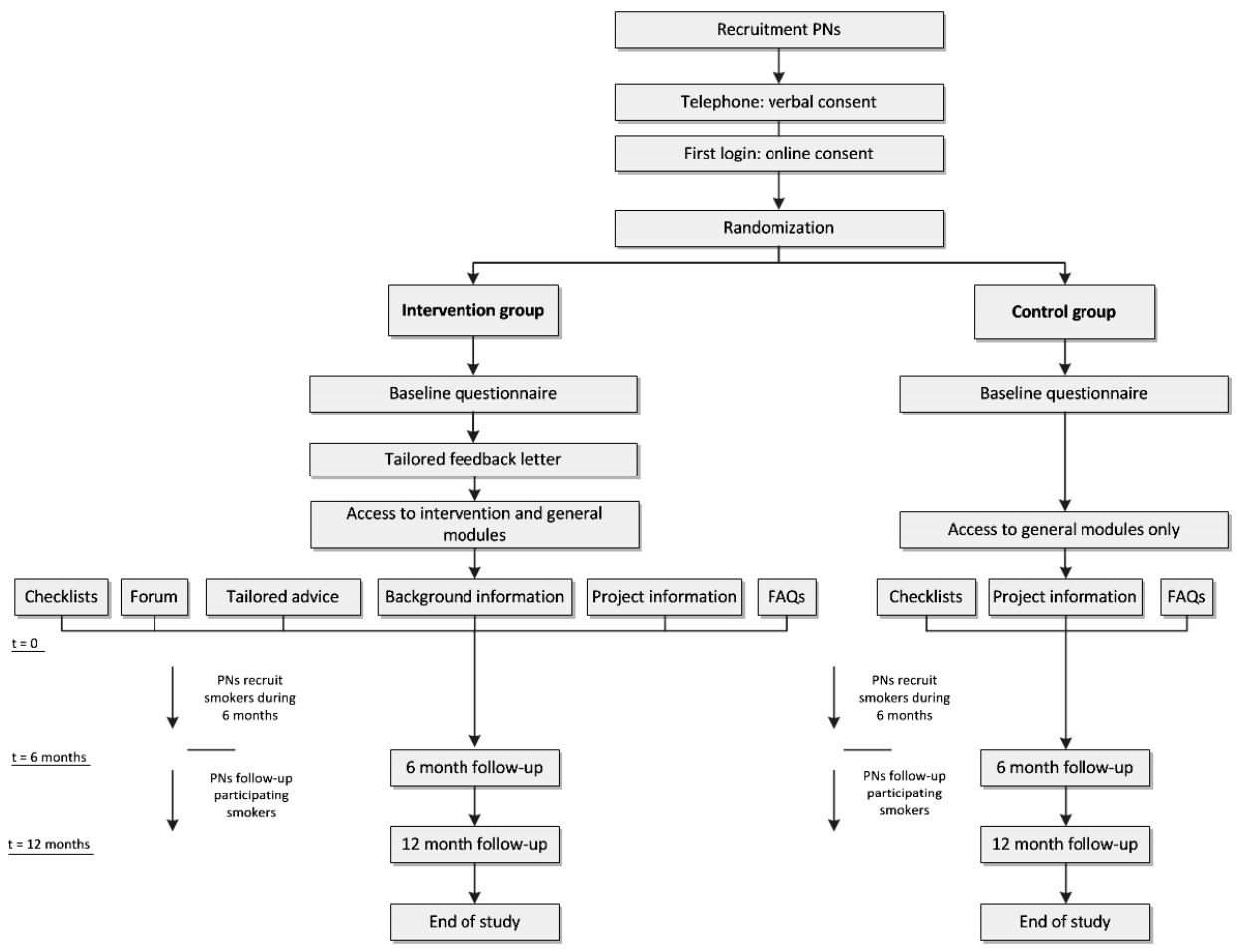

Figure 1 Flowchart of the design of the randomized effectiveness trial

\section{Recruitment of practice nurses}

PNs actively engaged in providing smokers with cessation counseling and working in general practices in the Netherlands are invited to participate in the trial. Moreover, PNs are 
eligible when they are sufficiently proficient in Dutch, have Internet access and have an active email account.

PNs who already participated in our preliminary studies (i.e. individual interviews with PNs and an online questionnaire for PNs) and reported to be interested in participation are personally invited to participate. Further recruitment takes place via several national institutes and organizations for Dutch PNs specifically, or primary care professionals more generally. Through these institutes and organizations PNs are initially invited via email, newsletters and website messages. Additionally, social media platforms such as Linkedln, Twitter and Facebook are used to inform as many people as possible about the research project and refer them to the project website (www.sterstudie.nl). This website has been online since September 2014, is free to visit and offers more details about the rationale and the different studies of the project, about the research team and about evidencebased smoking cessation guidelines in general. Through the website, PNs are also informed about the research objectives, the randomization procedure of the trial and the incentive for trial completion (i.e. a $€ 50$ gift voucher). They also have the opportunity to contact the research team for more information or directly register for participation in the trial via the website. In addition, a FAQs section is available on the website to directly answer questions that PNs might have. In case PNs report to be interested in participation they are subsequently contacted by telephone.

\section{Randomization}

After verbal consent is provided via telephone, PNs receive an email containing detailed information about the project and instructions for signing in to the web-based CT adherence support program (i.e. personal username-password combination and a personalized auto-login link). No one but the research team is able to retrieve these login data. After PNs access the program and start filling out the baseline questionnaire they are asked to provide online informed consent, after which they are randomly allocated to either the intervention or the control group of the trial. Group allocation takes place at respondent level by means of a computer software randomization device, which allocates approximately half of the PNs to each group. Blinding of the PNs is not possible, since they are aware of their access to the different modules of the web-based CT program after they have completed the baseline questionnaire.

\section{Recruitment of smokers}

Recruitment of smokers is important for the evaluation of smokers' abstinence rates and number of quit attempts, which is a secondary aim of the trial. Every smoking patient who visits the general practice and receives smoking cessation care from a PN participating in the trial is invited by his/her PN to participate. PNs are provided with a recruitment letter (i.e. sent to the PN via email by the research team and accessible via the module with project information of the web-based program) that can be used to inform smokers about 
the trial. Individuals eligible for participation should smoke at the start of the study and should receive individual smoking cessation counseling from a PN who is enrolled in the study. Furthermore, smokers must be 18 years or older, sufficiently proficient in Dutch, have Internet access and have an active email account.

Interested smokers give their PN permission to provide the research team with the smoker's email address and date of birth. Subsequently, PNs can register smokers for participation in the trial via one of the general modules (i.e. the counseling checklist) of the web-based CT program. Within this module, PNs are asked to submit the smoker's email address and date of birth. This information is automatically processed by a software program to send registered smokers a personalized email invitation to participate in the trial. The content of these mails are similar for smokers that are recruited by intervention group and control group PNs. Smokers are hence unaware of their PN's group allocation. Subsequently, participating smokers receive similar automatic email invitations and reminders to fill out a total of three online questionnaires during the trial. The content of these questionnaires are also identical for all smokers, irrespective of their PN's group allocation. Completion of all questionnaires is rewarded with a $€ 10$ gift voucher.

PNs in both study groups use the counseling checklist to recruit smoking patients only during the first six months of the trial. PNs receive several phone calls and personalized emails during this period to stimulate them to recruit an adequate number of smokers. During the final six months (i.e. after the first follow-up questionnaire) PNs only fill out the checklist after consultations with smokers who already participate in the trial. Hence, no new smokers are recruited during this final period.

\section{Web-based CT adherence support program}

The content and design of the web-based CT program for PNs is based on the results from an earlier needs assessment among Dutch PNs (de Ruijter, Smit, de Vries, Goossens, et al., 2017). This study, in which semi-structured individual interviews were held to discover perceived facilitators and barriers to smoking cessation guideline adherence and identify PNs' needs concerning an adherence support program, showed that PNs perceived several psychological (e.g. low self-efficacy to increase the motivation of smokers and arrange follow-up consultations) and practical barriers (e.g. lack of high-quality brochures and information about compensation by different healthcare insurers) to guideline adherence. The involvement of a general practitioner and the use of visually attractive information brochures were perceived as facilitating factors by PNs. Finally, PNs were interested in a simple program, which is easily accessible and provides individually relevant content instead of large amounts of general information (de Ruijter, Smit, de Vries, Goossens, et al., 2017). Based on these results, a web-based CT adherence support program was developed, aimed to provide PNs with personalized and tailored content about evidence-based smoking cessation guidelines and their adherence to and perceptions about these guidelines. Personalized and tailored information is better at attracting and 
keeping the individual's attention (Kreuter \& Wray, 2003). Furthermore, PNs can directly print the content and save it on their computer. Finally, in order to make PNs' program usage more convenient, they are free to visit the program as frequently as they like and wherever and whenever they want.

\section{Usability testing}

Usability tests with behavior change experts $(\mathrm{N}=5)$ and $\mathrm{PNs}(\mathrm{N}=5)$ were conducted to identify flaws in the web-based CT program and potential improving alterations. Experts assessed the program's usability using the heuristic evaluation method (Jaspers, 2009). They assessed the program's lay-out and interaction structure according to a set of ten usability heuristics (Nielsen \& Mack, 1994). PNs used the think aloud method, which means that they were asked to visit all modules of the web-based CT program, while verbalizing their actions and thoughts (Jaspers, 2009). Based on the usability tests the following improving alterations to the program were made: adding more images to modules with a lot of text; adding 'return-to-home' buttons to all modules; providing instructions for using the print and save function and disabling pop-ups in the internet browser; and changing visual analogue answer scales to numerical scales. This resulted in a web-based CT program, integrated in the project website (www.sterstudie.nl), consisting of five intervention modules and three general modules that are described in the following sections.

\section{Intervention modules}

Only PNs that are enrolled in the intervention group of the trial have access to the five intervention modules of the web-based CT program: three modules with tailored advice (i.e. brief tailored advice, extended tailored advice, and new tailored advice), an online forum, and a module with background information. The aim and a PN's usage of each of the modules are described below.

\section{Brief tailored advice}

After completing the baseline questionnaire all PNs in the intervention group always receive a tailored feedback letter. The brief tailored advice module of the web-based CT program allows PNs to re-read this tailored feedback letter, which is based on PNs' individual answers to the questions in the online baseline questionnaire concerning their demographic characteristics, adherence to evidence-based smoking cessation guidelines and their scores on behavioral predictors (attitude, self-efficacy, social influences, knowledge, and action and coping planning).

The content of the letter is kept rather simple and visually attractive through the use of figures and graphs. First, PNs are shown a color-coded bar chart (i.e. red for poor, yellow for not yet sufficient and green for satisfactory) showing their adherence to the different guideline steps during smoking cessation trajectories of their last ten smoking patients, followed by tailored recommendations for optimal adherence. Next, PNs see a 
similar color-coded chart that reports back their individual scores concerning behavioral predictors. This information is then followed by tailored pieces of advice providing PNs with brief explanations for their red, yellow or green scores for every behavioral predictor (attitude, self-efficacy, social influences, knowledge, and action and coping planning). Finally, the tailored feedback letter concludes with tailored recommendations for individual PNs to visit specific other intervention modules of the web-based CT program, and to select particularly those topics where there is room for improvement (i.e. red or yellow scores). For example, a PN who perceives many disadvantages of using an evidencebased smoking cessation guideline, as reported in the baseline questionnaire, is advised to visit the module 'extended tailored advice' and choose the topic 'disadvantages of guideline use'. In this module, additional tailored advice is provided aimed to invalidate those particular disadvantages of guideline use that are perceived by this specific PN (see extended tailored advice for an example).

\section{Extended tailored advice}

This module provides PNs with additional, more extensive tailored advice based on their answers to the questions in the online baseline questionnaire. Whereas the brief tailored advice module contains advice tailored on a more general level (e.g. addressing PNs' attitude by reflecting on how many advantages and disadvantages of guideline use they perceive), the extended tailored advice module provides more in-depth advice on a chosen topic. PNs can choose from a drop-down list about which topic(s) they would like to receive more extended tailored advice. The following topics can be chosen:

1. Guideline adherence; with advice distinguishing between guideline steps that an individual PN either satisfactorily, not yet sufficiently or poorly reported to adhered to.

2. Knowledge of smoking cessation guidelines; with advice on guideline elements about which an individual PN does not yet have sufficient knowledge. This was assessed with true-false items, so PNs only receive advice about wrongly answered items.

3. Perceived disadvantages of using a smoking cessation guideline; with advice aimed to invalidate disadvantages that an individual PN reported to perceive.

4. Perceived advantages of using a smoking cessation guideline; with advice aimed to endorse advantages that an individual PN reported to not yet perceive.

5. Perceived social influence to use a smoking cessation guideline; with advice distinguishing between how much (i.e. low, medium, high) social modeling, social norms and social support an individual PN reported to perceive.

6. Perceived self-efficacy to use a smoking cessation guideline in potentially difficult situations; with advice on dealing with only those situations that an individual PN perceived to be difficult.

7. Action planning; with advice on how individual PNs can prepare for smoking cessation consultations and use a smoking cessation guideline by making specific action plans (e.g. printing an overview of the guideline prior to a consultation). PNs are only asked to make plans for those steps they wish to apply in their counseling. 
8. Coping planning; with advice on how PNs can deal with difficult situations to use a smoking cessation guideline by making coping plans. Individual PNs only receive advice on situations they reported to be difficult.

These topics can be chosen one at a time, but PNs can choose a new topic and read the related advice as many times as they like. For example, a PN can select the topic 'disadvantages of guideline use' to receive tailored advice aimed to invalidate the individually perceived disadvantages of guideline use. For instance, if a PN perceives that adhering to a guideline is time-consuming, as indicated in the baseline questionnaire, the following advice is presented:

If you have recently started using a guideline, it is indeed possible that this can be more time-consuming at first. You need some time to get used to the structure of the guideline and to become familiar with the content of the different guideline steps. However, you will notice that, after working with it for a while, you are actually able to save some time. Using the guideline will become habitual, which results in working more efficiently during a smoking cessation consultation.

New tailored advice

This module provides PNs with new tailored advice and is especially designed to provide advice when a PN's behavior or behavioral determinants have changed since the last time they used the program. PNs are advised to first read the modules with brief and extended tailored advice before starting to use this module. Advice in this module is similar to the advice in the module with extended tailored advice, but is updated in order to match the updated personal situation of a PN, since the last time they used the program. For this advice to be generated, PNs need to answer the questions about the subject of their choice again, which are similar to the questions of the baseline questionnaire. From a drop-down list, they can choose about which topics they would like to receive new advice and subsequently fill out the corresponding questions about this topic. Then, new tailored advice is generated based on the answers they have provided. As in the extended tailored advice module, topics can be chosen one at a time, but PNs are free to obtain new feedback on all topics and use this module as many times as they like.

Online forum

This module provides PNs with the opportunity to contact colleagues via an online forum to discuss issues and ask questions related to smoking cessation guidelines and consultations. PNs are free to use the forum as they like, but several threads (i.e. smoking cessation counseling, smoking cessation guidelines, motivating smokers to quit) are created in advance by the research team to provide PNs with some structure when visiting the forum for the first time. Members of the research team moderate the forum, meaning that inappropriate or incorrect messages on the forum can be commented on or deleted. 


\section{Background information}

This module provides PNs with up-to-date information on a variety of topics relevant for smoking cessation counseling. The information includes facts and figures about smoking (cessation), Dutch rules and regulations about smoking, an overview of compensation of smoking cessation counseling by Dutch healthcare insurers, a description of the content of evidence-based smoking cessation guidelines, patient-centered materials that can be used during counseling, information about smoking related to pregnancy, and a collection of links to relevant websites for more information.

\section{General modules}

Both PNs that are enrolled in the intervention and control group of the trial have access to three general modules of the web-based CT program: project information, FAQs and the counseling checklist.

The module with project information provides general information about the research project and the web-based CT program. It also addresses aspects related to the randomized controlled trial, such as the timing of the invitations for the questionnaires, the randomization procedure, the recruitment of smokers and the incentive for trial completion.

The FAQs module provides PNs with answers to predefined questions regarding the web-based CT program and the randomized controlled trial. PNs can use this module to find immediate answers to relatively simple questions, such as 'How do I sign in to the online program?'. They can also directly contact the research team via email or telephone in case they have a question that is not covered by the FAQs module.

Finally, PNs in both groups have access to the counseling checklist. This checklist has two main goals, which are important for the randomized controlled trial testing the effectiveness of the web-based CT program. The checklist is a tool for PNs for a) the recruitment of interested smokers (see recruitment smokers) and b) registration of a PN's adherence to evidence-based smoking cessation guidelines (see data collection).

\section{Data collection}

PNs in both the control and the intervention group are prompted via email to complete three online questionnaires: at baseline (maximum 30 minutes required), and six months (maximum 20 minutes required) and 12 months (maximum 10 minutes required) after baseline. Email reminders are sent one week and two weeks after receiving the invitation to complete a questionnaire, each time a PN has not yet (completely) filled out that particular questionnaire. Questionnaires are based on the I-Change Model (de Vries et al., 2003) and the Self-Determination Theory (Deci \& Ryan, 1985), and are based on questionnaires previously used among health professionals (Bolman et al., 2002; Segaar, Willemsen, et al., 2007; Smit et al., 2010). The baseline questionnaire for PNs consists of 
questions concerning demographic characteristics (e.g. age, sex, education, work experience), smoking cessation guideline adherence, and potential behavioral predictors (i.e. intention, knowledge, attitude, self-efficacy, social influence, action and coping planning), as derived from the I-Change Model (de Vries et al., 2003). Furthermore, the questionnaire includes a set of questions about PNs' motivation to use an evidence-based smoking cessation guideline, based on Self-Determination Theory (Deci \& Ryan, 1985; Levesque et al., 2007). The 6-month follow-up questionnaire again includes questions about smoking cessation guideline adherence, potential behavioral predictors and motivation. Additionally, several questions are added to this questionnaire to inform the process evaluation, based on previously used questionnaires (Voncken-Brewster et al., 2013; Walthouwer, Oenema, Candel, Lechner, \& de Vries, 2015). The 12-month follow-up questionnaire includes questions concerning smoking cessation guideline adherence and the most proximal predictors of behavior (i.e. intention, attitude, self-efficacy, social influence) according to the I-Change Model (de Vries et al., 2003). Additionally, all PNs are asked whether they have had access to the intervention modules (i.e., brief tailored advice, extended tailored advice, new tailored advice, the online forum and background information) during the trial, as to corroborate that PNs in the control group were not exposed to intervention modules.

Smoking patients counseled by PNs from both groups are invited via email to fill out three online questionnaires (maximum 10 minutes required per questionnaire): at baseline, and 6 months and 12 months after baseline. The baseline questionnaire includes questions concerning demographic characteristics (e.g. age, sex, and education), smoking status, their PN's smoking cessation guideline adherence and autonomy-supportiveness, based on Self-Determination Theory (Deci \& Ryan, 1985; Jochems, Mulder, Duivenvoorden, van der Feltz-Cornelis, \& van Dam, 2014). Moreover, healthcare usage, and quality of life (Al-Janabi, Flynn, \& Coast, 2012; Janssen et al., 2013) are measured. The 6-month and 12-month follow-up questionnaires again address a patient's smoking status, their PN's smoking cessation guideline adherence and autonomy-supportiveness, and a patient's healthcare usage and quality of life. Additionally a few questions are included to inform the evaluation of PNs' smoking cessation consultations with patients (e.g. assessing whether a patient agrees/disagrees about their PN's advice being helpful to quit smoking).

As briefly mentioned in the description of the general modules, data about PNs' smoking cessation guideline adherence is also collected via the counseling checklist. During the entire trial period (i.e. twelve months) PNs in both groups provide smoking cessation care to their patients and are asked to fill out a counseling checklist after every consultation with a patient. Via the counseling checklist, PNs fill out the date and duration of each smoking cessation consultation, and report which guideline steps were adhered to (yes/no) during that particular consultation. The following guideline steps are assessed in the checklist: providing a quit advice, assessing smoking profile and smoking history, assessing motivation, increasing motivation, assessing barriers, discussing relevant barriers, 
discussing the use of cessation aids, setting a quit date and making a quit plan, and organizing consultations after the quit date. Finally, PNs can briefly describe why particular steps were not adhered to, if this was the case. When PNs fill out a checklist about the next consultation with the same patient they can select the email address of that particular smoker from a drop-down menu. This enables the researchers to register the number of consultations with each patient.

\section{Outcome measures}

The primary outcome measure of this randomized controlled trial is PNs' evidence-based smoking cessation guideline adherence, which is calculated based on three assessments:

1. By assessing PNs' adherence as perceived by participating smokers (in smokers' baseline and both follow-up questionnaires).

2. By asking PNs to self-report their adherence to each of the guideline steps during each consultation with a smoking patient (using the counseling checklist, throughout the study).

3. By at random audiotaping $10 \%$ of the consultations during which PNs engage in smoking cessation counseling with a smoking patient (throughout the study, with audiotapes scored by two independent coders).

All data concerning PNs' smoking cessation guideline adherence is collected dichotomously: has each step been adhered to (yes/no)? This results in a score ranging from zero (none of the steps are adhered to) to nine (all steps are adhered to) for each type of assessment. PNs' self-report data and smokers' data will be triangulated and averaged for each counseled smoker (Reif, Horgan, \& Ritter, 2011). Subsequently, a mean adherence score will be calculated for each PN based on all consultations conducted during the trial period (12 months). This adherence score will be used as the primary outcome measure for this study. In case large discrepancies (i.e. a difference in adherence of 3 guideline steps) between the two self-report measures are observed, both PN and smoker endpoints will be used as separate outcome measures instead of using the triangulated score.

\section{Secondary outcome measures}

Secondary outcome measures are counseled smokers' point prevalence smoking abstinence and number of serious quit attempts during the trial period. Point prevalence abstinence (PPA) is measured by asking whether the participant has smoked (yes/no) during the last 24 hours (24h PPA) and during the last seven days (7d PPA). A serious quit attempt is defined as a period of at least 24 hours of abstinence. 


\section{Statistical analyses}

\section{Sample size and power calculation}

One of PNs' main tasks is to counsel patients wanting to change their lifestyle; therefore, it is hypothesized that at baseline most PNs already adhere to at least one of the smoking cessation guideline steps (i.e. provide quit advice). Due to measurement effects, an increase in adherence is expected in the control group to adherence to two steps, while it is estimated that the intervention yields adherence to four steps. To be able to detect this difference significantly ( $\alpha=5 \% ; \beta=10 \%$ ), assuming a medium effect size (Cohen, 1988), 95 PNs per arm are necessary at the end of the trial (190 in total). Considering 30\% attrition we aim to include at least 272 PNs at baseline.

Among smokers, a 10\% difference in 7d PPA is expected between smokers in the control group (expected increase of 10\%) (Hoving, Mudde, Dijk, \& de Vries, 2010; Te Poel, Bolman, Reubsaet, \& de Vries, 2009) and intervention group (expected increase of 20\%). To significantly ( $\alpha=5 \%$; $\beta=20 \%$ ) detect this difference, using an Intraclass Correlation Coefficient of 0.06, taking into account the multilevel design of the trial and a pessimistic scenario (i.e. allowing for variation in the number of recruited smokers by different PNs) (Candel \& Van Breukelen, 2010), the 190 PNs need to include around 1200 smokers (6-7 smokers each).

\section{Data analysis}

To assess the effects of the intervention and other potential predictors on adherence, linear regression analyses will be conducted. To assess effects on smoking cessation measures logistic (PPA variables) or linear (number of quit attempts) regression analyses will be done. Intention-to-treat (ITT) analysis as well as complete case analysis will be conducted. Data will be analyzed using SPSS version 21.

\section{Additional studies}

In addition to the described randomized controlled trial, several other studies will be conducted within this research project.

\section{Process evaluation}

As part of the first follow-up questionnaire, all PNs are asked about their general experiences with the web-based CT program, their opinion about the program's user-friendliness, and about possible improving alterations to the program. PNs in the intervention group also evaluate the extra modules they had access to during the trial period in terms of their usefulness and user-friendliness. Data gathered with these questions are used to develop an interview guide to conduct additional telephone interviews with PNs to obtain 
specific recommendations for improvement of intervention materials. The process evaluation among smokers predominantly evaluates the quality of their PN's smoking cessation care as perceived by the smokers.

\section{Forum usage analysis}

PNs' activities on the forum are monitored in order to conduct an analysis of forum usage. The following forum parameters are collected for every PN: number of visits, number of posts, number of replies and number of views. In addition, data is collected about the content of forum posts, and about interactions between forum users writing a particular post and users replying to those posts. An analysis of these parameters can be used to investigate whether the degree of forum usage and user interaction influences the study's outcome measures.

\section{Economic evaluation}

The economic evaluation study involves a combination of a cost-effectiveness analysis (CEA) and a cost-utility analysis (CUA). Data for this study is collected during the baseline and follow-up measurements as part of the trial. For both CEA and CUA the intervention costs, healthcare costs and patient costs are identified as relevant. For the CEA, the incremental cost-effectiveness ratio (ICER) is expressed as the incremental costs per additional quitter (measured as 7d PPA) in the intervention group as compared with the control group. For the CUA, the ICER is expressed as the incremental costs per quality-adjusted life year (QALY) gained, based on the quality of life measures EuroQol (Janssen et al., 2013) and ICECAP (Al-Janabi et al., 2012).

\section{Discussion}

This paper described the development of a web-based CT adherence support program for Dutch PNs and the study design of a randomized controlled trial testing its effectiveness. It is hypothesized that CT advice can support PNs to improve their adherence to evidence-based smoking cessation guidelines during consultations with their smoking patients. Due to the tailored nature of the web-based advice, PNs only receive advice and practical tips about counseling aspects they have individually identified as being relevant to them. Furthermore, by taking into account PNs' needs regarding the design and content of the web-based CT support program, an easy-to-use program was created containing individually relevant and visually attractive information which PNs can use at their convenience wherever and whenever they want. Incorporating PNs' content and design needs was hypothesized to increase their interest in the program resulting in higher exposure to the program's content (de Vries \& Brug, 1999; Eysenbach, 2005; Schneider et al., 2012) and making it more likely that the program actually improves PNs' smoking cessation guideline adherence. PNs' improved guideline adherence could subsequently 
enhance the quality of smoking cessation care in Dutch general practices, ultimately resulting in a higher number of smokers successfully quitting smoking. If proven effective, it might hence be worthwhile to pursue widespread implementation of web-based CT adherence support for PNs, leading to increased benefits regarding the amount of smoking related illness and death.

\section{Potential strengths of the study}

This study has several potential strengths. First, triangulation is used for calculating our primary outcome measure. PNs' evidence-based guideline adherence is assessed via online questionnaires filled out by counseled smokers, via self-report questionnaires filled out by PNs (checklists), and via an assessment of audiotaped consultations by two independent coders. Assessing the same outcome measure from three different perspectives and subsequently integrating them into a single outcome measure improves the reliability of our primary outcome measure (Moran-Ellis et al., 2006). In this way, the influence of responding in a socially desirable manner, which is often observed in self-report questionnaires (Van de Mortel, 2008), can be corrected for by assessments via smoking patients and audiotaped consultations with these patients.

Secondly, the design and content of the web-based CT program is based on theory (de Vries et al., 2003; Deci \& Ryan, 1985), previously developed CT programs (Bolman et al., 2002; Segaar, Willemsen, et al., 2007; Smit et al., 2010) as well as on a qualitative needs assessment we conducted among Dutch PNs (de Ruijter, Smit, de Vries, Goossens, et al., 2017). This helped us to match the content of the CT program to the most important factors influencing PNs' guideline adherence and to develop an adherence support program that matches PNs' needs concerning content and design features of such a program. Integrating PNs' needs in the design of the web-based CT program is likely to enhance their interest in and use of the program, aiming for maximum exposure to the program's tailored content (Schneider et al., 2012) and resulting in optimal effectiveness.

Thirdly, random allocation of PNs to either the control or intervention group is executed by an automated computerized process. The research team has no influence on this process since they only know in which group a participant is placed after the allocation procedure has finished.

\section{Potential limitations of the study}

The present study also has some potential limitations. First, randomization on PN level might lead to PNs from the same general practice being allocated to different treatment groups. This might introduce contamination, since information transfer between different PNs could take place (i.e. from an intervention group PN to a control group PN, and vice versa). However, in only $22,4 \%$ of Dutch general practices more than one PN is employed (Heiligers et al., 2012), which restricts the potential for contamination bias to only these practices. Moreover, by asking PNs in both study groups at the second follow-up 
measurement whether they have had access to intervention group modules (i.e., brief tailored advice, extended tailored advice, new tailored advice, the online forum and background information) during the trial we are able to measure exposure to these modules - also among control group PNs. Potential access to these intervention modules by PNs in the control group can subsequently be corrected for in statistical analyses.

Secondly, participating PNs know about group allocation after they have filled out the baseline questionnaire, because they see what modules of the CT program they have access to at that moment. PNs who are allocated to the intervention group might be more likely to adhere to filling out counseling checklists, to recruit smokers and report on the consultations with smokers held during the intervention period, than PNs in the control group, because they know they have full access to all modules. In order to promote the use of the counseling checklists among all PNs, for recruiting smokers and reporting on consultations, email reminders are sent to all PNs stressing the importance of using the checklist and providing tailored feedback on how often they have used it so far. Moreover, as an extra stimulus to engage with the online program, PNs in the control group are informed that they receive access to the content of the intervention module with background information after the trial has ended. These strategies are implemented to prevent high rates of non-usage attrition, especially among PNs in the control condition (Eysenbach, 2005).

A third potential limitation concerns the high rates of drop-out attrition among participants in web-based interventions, often caused by a lack of face-to-face contact and a high degree of anonymity (Eysenbach, 2005). In the present study, we try to prevent high attrition rates among participating PNs with several strategies often applied in webbased CT programs, such as providing tailored feedback, sending reminders via email, providing an incentive for study completion, minimizing usability challenges and providing personal contact (Bennett \& Glasgow, 2009). By integrating PNs' needs regarding the content and design of the web-based CT program as identified in our preliminary needs assessment, we aim to make the program as personally relevant and convenient to use as possible. Moreover, usability challenges are minimized by incorporating results from usability tests among PNs and behavior change experts, which were conducted prior to the start of the trial. Finally, personal contact is ensured by contacting PNs via telephone at three time points during the trial, in order to lower the degree of anonymity and make sure PNs know the researchers involved in the study and also how to be able to contact them with problems or questions. Relatedly, in order to prevent high rates of drop-out attrition among smokers, we use strategies like minimal time investment, email reminders and an incentive for study completion (Bennett \& Glasgow, 2009). 


\section{Conclusion}

This detailed description of the development of a web-based CT adherence support program for Dutch PNs offers insight into its potentially effective working mechanisms. The results of the randomized controlled trial testing the program's (cost-)effectiveness, the process evaluation study and an analysis of forum usage will be reported in other papers. These results will benefit our understanding of effective interventions supporting PNs working in the general practice setting to improve their adherence to evidence-based smoking cessation guidelines. Furthermore, when proven effective, the results from these studies might provide insight into the potential value of adapting the program's content to the work situation of other healthcare professionals involved in smoking cessation counseling. Adherence to evidence-based smoking cessation guidelines among health professionals such as midwives, dentists and physical therapists is not yet optimal either and could hence also be improved (Johnson, Lowe, \& Warnakulasuriya, 2006; Stop Smoking Partnership, 2009). Adaptation and implementation of an effective web-based CT adherence support program for a wider range of health professionals could lead to even greater public health benefits. 


\section{Chapter}

The effectiveness of a computer-tailored e-learning program for practice nurses to improve their adherence to smoking cessation counseling guidelines: randomized controlled trial 


\begin{abstract}
Background: Improving practice nurses' (PNs') adherence to smoking cessation counseling guidelines will benefit the quality of smoking cessation care and will potentially lead to higher smoking abstinence rates. However, support programs to aid PNs in improving their guideline uptake and adherence do not exist yet. The aim of this study was to assess the effects of a novel computer-tailored e-learning program on PNs' smoking cessation guideline adherence.
\end{abstract}

Methods: A Web-based randomized controlled trial (RCT) was conducted in which an intervention group ( $\mathrm{N}=147)$ with full access to the e-learning program for 6 months was compared with a control group ( $\mathrm{N}=122)$ without access. Data collection was fully automated at baseline and 6-month follow-up via online questionnaires, assessing PNs' demographics, work-related factors, potential behavioral predictors based on the I-Change model, and guideline adherence. PNs also completed counseling checklists to retrieve self-reported counseling activities for each consultation with a smoker ( $N=1175)$. To assess the program's effectiveness in improving PNs' guideline adherence (i.e. overall adherence and adherence to individual counseling guideline steps), mixed linear and logistic regression analyses were conducted, thus accommodating for the smokers being nested within PNs. Potential effect moderation by work-related factors and behavioral predictors was also examined.

Results: After 6 months, 121 PNs in the intervention group (82.3\%, 121/147) and 103 in the control group $(84.4 \%, 103 / 122)$ completed the follow-up questionnaire. Mixed linear regression analysis revealed that counseling experience moderated the program's effect on PNs' overall guideline adherence (beta=.589; 95\% Cl 0.111-1.068; $P$ Holm-Bonferroni $=.048$ ), indicating a positive program effect on adherence for PNs with a more than average level of counseling experience. Mixed logistic regression analyses regarding adherence to individual guideline steps revealed a trend toward moderating effects of baseline levels of behavioral predictors and counseling experience. More specifically, for PNs with less favorable scores on behavioral predictors (e.g. low baseline self-efficacy) and high levels of counseling experience, the program significantly increased adherence.

Conclusions: Results from our RCT showed that among PNs with more than average counseling experience, the e-learning program resulted in significantly better smoking cessation guideline adherence. Experienced PNs might have been better able to translate the content of our e-learning program into practically applicable counseling strategies compared with less experienced colleagues. Less favorable baseline levels of behavioral predictors among PNs possibly contributed to this effect, as there was more room for improvement by consulting the tailored content of the e-learning program. To further substantiate the effectiveness of e-learning programs on guideline adherence by health care professionals (HCPs), it is important to assess how to support a wider range of HCPs. 


\section{Introduction}

Smoking is the most preventable cause of illness and premature death worldwide (USDHHS, 2014; WHO, 2015). In the Netherlands, 24.1\% of adults still smoked in 2016 (Springvloet \& van Laar, 2017), illustrating the persistent need for effective smoking cessation strategies. For example, the general practice setting has great potential for cessation support, as over $75 \%$ of Dutch smokers visit their general practice at least once a year (Kleinjan et al., 2016). General practice health care professionals (HCPs) such as practice nurses (PNs) and general practitioners (GPs) are trained to use evidence-based smoking cessation guidelines (i.e. the STIMEDIC guideline (Trimbos-instituut \& NHG, 2016) being the most recent one) to counsel their smoking patients. Applying such guidelines in structured cessation treatment, which combines behavioral and pharmacological support (Trimbos-instituut \& NHG, 2016), is known to have beneficial effects on smokers' abstinence rates (Stead et al., 2013). However, only in $25 \%$ to $33 \%$ of consultations do smokers receive a quit smoking advice in their general practice (Kleinjan et al., 2016). Moreover, once a quit advice has been given, more extensive smoking cessation support should be provided, which is most often the responsibility of PNs (Freund et al., 2015). Yet, also concerning subsequent steps of evidence-based smoking cessation guidelines, PNs' adherence is suboptimal (de Ruijter, Smit, de Vries, \& Hoving, 2017). Consequently, improving PNs' guideline adherence would benefit the quality of the smoking cessation care in the general practice and could therefore lead to higher smoking abstinence rates (Miller \& Kearney, 2004; Rice et al., 2013).

An earlier study investigating PNs' needs for guideline adherence support found that they were interested in an individually relevant, easy-to-use, and practically applicable program or intervention (de Ruijter, Smit, de Vries, Goossens, et al., 2017). Moreover, research showed that PNs' guideline adherence is positively related to their level of selfefficacy for using a guideline and perceiving advantages of using a guideline (Berndt et al., 2013; de Ruijter, Smit, de Vries, \& Hoving, 2017; Segaar, Willemsen, et al., 2007; Studts et al., 2010). Such behavioral predictors could be targeted through intervention programs aimed to improve PNs' guideline adherence. More specifically, providing PNs with content tailored to behavioral predictors fulfills their need for an individually relevant program, and therefore, tailored content is more likely to be read and remembered, compared with nontailored program content (Kreuter \& Wray, 2003). For instance, tailored content can be matched with PNs' individual level of self-efficacy and their perceived advantages of guideline usage: information can be provided regarding potentially difficult counseling situations (e.g. when limited time is available) or regarding specific benefits of using a smoking cessation guideline during consultations (e.g. increasing counseling quality), as identified by each individual PN in an earlier evaluation (de Vries \& Brug, 1999).

Additionally, providing PNs with online access to tailored content (i.e. computer-tailored, CT) enables them to consult it time-efficiently and whenever and wherever they 
desire (Griffiths et al., 2006; Tate et al., 2009). Previously tested CT programs proved to be effective in changing various (determinants of) health behaviors, including smoking cessation (Smit, Candel, Hoving, \& de Vries, 2016; Taylor et al., 2017). Therefore, by targeting PNs' behavioral predictors via a Web-based CT support program, positive behavior change can be achieved among PNs, meaning that they improve their smoking cessation guideline adherence. Moreover, despite PNs' interest in tailored adherence support (de Ruijter, Smit, de Vries, Goossens, et al., 2017), such (Web-based) CT programs do not yet exist with the aim to increase PNs' smoking cessation guideline adherence. Therefore, we developed and tested a novel Web-based CT e-learning program for PNs to support them to improve their smoking cessation counseling guideline adherence (de Ruijter et al., 2016).

The aim of the study described here was to assess the effects of the CT e-learning program on PNs' smoking cessation guideline adherence in a randomized controlled trial (RCT). We hypothesized that PNs' guideline adherence would significantly improve as a result of exposure to the $\mathrm{CT}$ e-learning program.

\section{Methods}

\section{Study design}

We conducted an RCT to investigate the effectiveness of the CT e-learning program on PNs' smoking cessation guideline adherence, compared with no intervention. A full description of the design of the RCT can be found elsewhere (de Ruijter et al., 2016). Evaluation by the Medical Ethics Committee Atrium-Orbis-Zuyd (14-N-17) revealed that no medical ethical clearance for this study was needed according to the rules of the Medical Research Involving Human Subjects Act (WMO). The study is registered with the Dutch Trial Register (NTR4436).

\section{The computer-tailored e-learning program}

The CT e-learning program was structurally based on previously developed CT programs (Smit et al., 2010; Stanczyk et al., 2011) and consisted of (1) Several e-learning modules in which PNs had access to individually tailored advice, a forum, and smoking cessation counseling materials (both to inform themselves and to provide to smokers) and (2) Three general modules with project information, frequently asked questions about the RCT, and a counseling checklist to monitor self-reported counseling activities during the trial (de Ruijter et al., 2016). The content of advice modules was tailored to several respondent characteristics theoretically grounded in the I-Change Model (ICM (de Vries, 2017)), which were previously demonstrated to be effective in achieving behavior change (Bolman et al., 2015; Elfeddali et al., 2012; Smit et al., 2012; Stanczyk, Bolman, et al., 
2014): demographics (e.g. gender), premotivational factors (e.g. knowledge), motivational factors (e.g. self-efficacy), postmotivational factors (e.g. coping planning), intention (to use a smoking cessation guideline), and behavior (i.e. self-reported application of smoking cessation guideline steps).

\section{Participants and procedure}

PNs across the Netherlands were contacted through email, newsletters, and website messages via national organizations for PNs or primary care professionals in general, as well as via a project website (de Ruijter, Smit, De Vries, \& Hoving) and social media platforms (i.e. Twitter, LinkedIn, and Facebook). Additionally, individual PNs were contacted by the research team via telephone through their general practice. Eligible PNs were actively engaged in smoking cessation counseling in a Dutch general practice, had Internet access and an active email account, and were sufficiently proficient in Dutch. Upon interest and obtaining important project information via telephone and email, PNs were prompted to visit the $\mathrm{CT}$ e-learning program to complete an online informed consent form, were randomized (i.e. allocation by a computer software randomization device), and were asked to fill out the Web-based baseline questionnaire. As PN enrollment in the trial was spread over a period of 6 months, randomization was conducted at respondent level at the time of enrollment of an individual PN.

Individual PNs who were randomly allocated to the intervention group of the trial had access to all e-learning and general modules described above and received a tailored feedback letter based on their answers to the baseline questionnaire; this letter provided individual PNs with a summary of various pieces of tailored advice (i.e. on different motivational factors and behavior) and instructions on where to find more elaborate advice in the e-learning modules. PNs in the control group only had access to the general modules. During a 6-month time period (i.e. upon completion of the baseline questionnaire), PNs in the intervention and control group were free to visit the modules of the CT elearning program that were available to them based on their group allocation as many times as they wanted. PNs could directly print content from the modules and save this content on their computer.

During the trial, PNs in both the intervention and control group were asked to engage in smoking cessation counseling with their smoking patients when the opportunity arose. All PNs were asked to recruit these smokers to partake in the trial (Figure 1). When smokers agreed, PNs were instructed to record smokers' date of birth and email address. Smokers then directly received an email invitation to participate in the trial and to fill out an online questionnaire. 


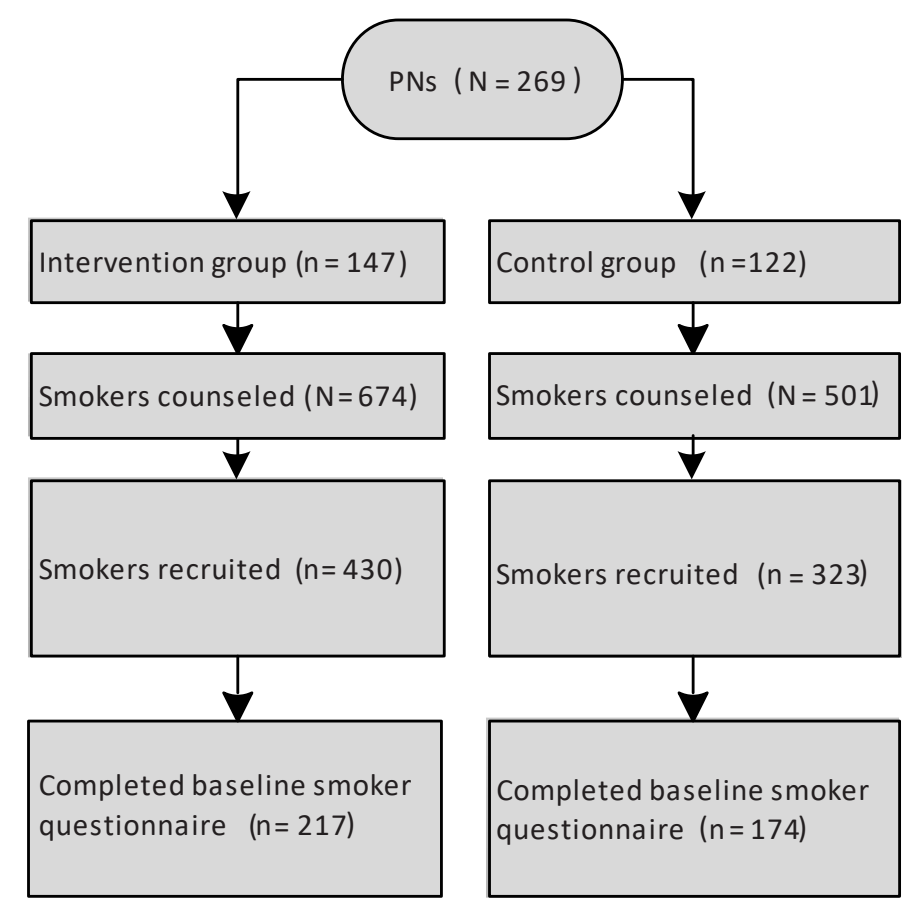

Figure 1 Flow of counseled smokers recruited by practice nurses

\section{Data collection among practice nurses}

Baseline and 6-month follow-up questionnaires for PNs were informed by the ICM (de Vries, 2017) and were based on questionnaires previously used among HCPs to assess smoking cessation activities (Bolman et al., 2002; Segaar, Willemsen, et al., 2007; Smit et al., 2010). Questionnaires were identical for intervention and control group PNs and administered in a Web-based format. The baseline questionnaire for PNs consisted of questions concerning demographic characteristics, potential behavioral predictors of adherence, and their guideline adherence. The follow-up questionnaire included the same questions about potential behavioral predictors and PNs' guideline adherence. Additional data on PNs' smoking cessation guideline adherence were collected via the counseling checklist that PNs filled out after each consultation with a smoker throughout the 6month intervention period and during a 6-month follow-up period.

\section{Demographics}

After providing online informed consent, every PN was requested to fill out their first and last name, gender, date of birth, and smoking status (smoker, ex-smoker, nonsmoker). Subsequently, they filled out in how many general practices they worked, how many hours they worked per week, and whether or not they were listed in the Dutch Stop Smoking Quality Register (i.e. a register with qualified smoking cessation professionals). The final 
questions concerned the practice in which a PN worked most hours per week; they filled out practice name, experience in smoking cessation counseling in years, the presence of designated smoking cessation consulting hours (yes or no), and whether patients' smoking status was systematically registered in their patient files (yes or no).

\section{Behavioral predictors}

Several socio-cognitive factors were assessed as potential predictors of guideline adherence, informed by the ICM (de Vries, 2017): intention, knowledge, attitude, self-efficacy, social influence, action planning, and coping planning. Items were based on previously used questionnaires about behavioral predictors related to nurses' smoking cessation counseling (Bolman et al., 2002; Segaar, Willemsen, et al., 2007; Smit et al., 2016).

PNs' intention to use a smoking cessation guideline was assessed by two questions addressing the intention to use (1) Any evidence-based smoking cessation guideline and (2) The most recent Dutch counseling protocol, specifically (i.e. STIMEDIC guideline (Trimbos-instituut \& NHG, 2016): Do you intend to use the STIMEDIC quit smoking guideline?), using the same answering scale (1=definitely not, 4=do not know, 7=definitely).

PNs' knowledge of evidence-based smoking cessation guidelines was assessed by 18 true-false items concerning the content of the STIMEDIC guideline (e.g. the first consultation of a smoking cessation trajectory starts with providing a quit advice). As such, PNs scored points for every statement they appropriately identified to correctly reflect the content of the counseling protocol (range: 0-18).

PNs' attitude was assessed by seven items about perceived advantages (e.g. using an evidence-based guideline improves the quality of my smoking cessation counseling) and seven items about perceived disadvantages (e.g. using an evidence-based guideline is time-consuming for me) of using an evidence-based smoking cessation guideline ( $1=$ completely disagree, $5=$ completely agree). These items were subsequently combined into separate scales for perceived advantages (Cronbach alpha=0.82; $\Omega=0.82$ ) and perceived disadvantages (Cronbach alpha=0.74; $\Omega=0.74$ ).

PNs' level of self-efficacy was assessed by ten items describing potentially difficult situations when trying to adhere to an evidence-based smoking cessation guideline (e.g. when it is very busy at the general practice) and asking PNs how difficult they would find it to follow a guideline in each of these situations ( $1=$ very difficult, $5=$ very easy). All items were combined into a self-efficacy scale (Cronbach alpha $=0.84 ; \Omega=0.84$ ).

PNs' perceived social influence was assessed by three items about social modeling (e.g. the GP works with an evidence-based smoking cessation guideline), five items about social support (e.g. colleagues in other practices support the use of an evidence-based smoking cessation guideline), and five items about social norms (e.g. the practice manager thinks using an evidence-based smoking cessation guideline is important). All items assessed the potential influence of important others within and outside the general practice (1=completely disagree, $3=$ neutral, 5=completely agree) and were subsequently combined into separate scales for social modeling (Cronbach alpha=0.62; $\Omega=0.63$ ), social 
support (Cronbach alpha=0.73; $\Omega=0.74$ ), and social norms (Cronbach alpha=0.71; $\Omega=0.72$ ). Scores that represented a not applicable answering category were assigned a neutral score.

PNs' intention to make action plans and coping plans was assessed by eight and ten items, respectively (yes or no). Action plans addressed specific activities for preparing a smoking cessation consultation with a patient (e.g. discussing a patient's smoking status with the GP), whereas coping plans addressed their aspiration to develop a concrete plan for dealing with potentially difficult situations (i.e. plans for dealing with the same potentially difficult situations as assessed in the self-efficacy questions). Subsequently, sum scores for both action plans (range: 0-8) and coping plans (range: 0-10) were computed.

\section{Guideline adherence}

Questions on guideline adherence concerned the nine evidence-based counseling steps, as described in the STIMEDIC guideline (Trimbos-instituut \& NHG, 2016): (1) advising to quit smoking, (2) assessing smoking profile and smoking history, (3) assessing motivation to quit, (4) increasing motivation, (5) assessing barriers to quitting, (6) discussing barriers, (7) informing about cessation aids, (8) making a quit plan and setting a quit date, and (9) arranging follow-up after the quit date. PNs' adherence at baseline was assessed by asking PNs to self-report their adherence to each guideline step (e.g. I advised my patient to quit smoking; step 1) during complete smoking cessation trajectories (i.e. intake and follow-up consultations) of their last ten patients (range: 0-10). These data on PNs' adherence were used to create $\mathrm{CT}$ advice for PNs in the intervention group regarding their behavior. In the effect analyses, PNs' baseline guideline adherence score was used as a covariate. Additionally, during the trial period, guideline adherence was assessed by asking PNs to self-report their adherence to each guideline step (i.e. Please select which subjects were addressed during the consultation with your smoking patient) after every consultation with a smoking patient (yes or no) using the counseling checklist (i.e. one of the general modules in the CT e-learning program available for PNs in both the intervention and control group). This resulted in a score from 0 (none of the steps were adhered to) to 9 (all steps were adhered to) for each individual consultation with a smoker. Checklists of consultations with the same smoker were combined into a single score for guideline adherence, reflecting a PN's adherence during a complete counseling trajectory of a smoking patient, which was the primary outcome measure in the effect analyses.

\section{Sample size calculation}

We calculated the required sample size based on the possibility to detect a difference of medium effect size (i.e. adherence to two additional guideline steps) between intervention and control group PNs (alpha=5\%; beta=10\%). As a result, at least 95 PNs per condition at the end of the trial would be sufficient (de Ruijter et al., 2016). However, to detect a medium effect size for an interaction with the intervention factor when assuming an intraclass 
correlation of .25, at least 105 PNs per condition are needed to ensure a statistical power of $80 \%$. Considering $30 \%$ attrition, we aimed to include 300 PNs at baseline.

\section{Statistical analyses}

Reliability analyses (i.e. Cronbach alpha and $\Omega$ ) were conducted using R version 3.4.0 ( $R$ Foundation for Statistical Computing), and other statistical analyses were conducted using SPSS version 23.0 (IBM Corp). Descriptive analyses were conducted to summarize PNs' characteristics, whereas independent-samples $t$ tests and chi-square tests determined significant $(P<.05)$ baseline differences between intervention and control group PNs. Logistic regression was used to determine selective dropout of PNs after baseline, including variables potentially related to PNs' guideline adherence (i.e. specific work-related variables, behavioral predictors, and baseline guideline adherence). On the basis of analyses for baseline differences and selective dropout, statistically significant variables were identified and included as covariates in further analyses.

As smokers were nested within PNs participating in the trial, mixed regression analyses were conducted to assess the effects of exposure to the CT e-learning program on PNs' smoking cessation guideline adherence. Both PNs' overall adherence score (range: 0-9) was used as outcome measure and their adherence score for each guideline step separately (i.e. step-based adherence; $0=$ nonadherent, $1=$ adherent). Therefore, both linear and logistic mixed models were run, including the same covariates. Effect moderators were tested by including interaction effects with PNs' group allocation (i.e. intervention or control) to the regression models tested. On the basis of literature, several work-related factors (i.e. counseling experience and presence of consulting hours (Fleuren et al., 2004; Sangster-Gormley et al., 2011)) and behavioral predictors (i.e. intention, attitude, self-efficacy, and social influence (de Ruijter, Smit, de Vries, \& Hoving, 2017; Leitlein et al., 2012; Smit, de Vries, et al., 2013)), potentially moderating the program's effect on PNs' adherence, were tested. First, nonsignificant interaction effects were stepwise deleted using a backward deletion procedure, meaning that at each step the least significant interaction effect was removed. Second, nonsignificant covariates were deleted from the model following the same procedure, with the restriction that these covariates remained in the model if they were also part of a significant interaction term. Upon finding a significant interaction effect, subsequent subgroup analyses were conducted to determine the nature of the moderation using adjusted alpha levels (Holm-Bonferroni method) to correct for multiple testing. For subgroup analyses, the final mixed regression model was repeated, while replacing the original moderator with three centered versions of the moderator, centered by subtracting the mean - $1 \mathrm{SD}$, the mean, and the mean $+1 \mathrm{SD}$ from the original scores on the moderating variable. This allows for testing the effects of the e-learning program for three subgroups: one group corresponding to a score of the mean - 1 SD on the moderating variable, a second group with a score at the average on 
the moderating variable, and a third group with a score at mean + 1 SD on the moderating variable.

As 211 PNs (78.4\%, 211/269) completed at least one checklist (which was needed to calculate the primary outcome measure), it meant that 58 PNs were excluded from effect analyses. For this reason, sensitivity analyses were conducted by replacing missing values on the primary outcome measure (guideline adherence) with scores assuming some dependency between the score being missing and the adherence score itself, either following an optimistic or a pessimistic scenario. In both scenarios, missing data were imputed for these 58 PNs based on the average number of patients counseled during the trial per PN. Furthermore, PNs' dropout status was taken into account, as some PNs, who did not complete any checklists, also did not fill out the follow-up questionnaire (i.e. PN dropouts). One might expect PNs who did complete the follow-up questionnaire (i.e. retained PNs) to be more motivated and to be more adherent if they would have completed the checklists during the intervention period. This was taken into account when imputing data. In the optimistic imputation scenario, retained PNs were assumed to be adherent in $90 \%$ of the consultations with smokers, whereas PN dropouts were assumed to be adherent in only $80 \%$ of their consultations. In the pessimistic scenario, a $50 \%$ probability of adherence was assumed for retained PNs and a $20 \%$ probability of adherence for PN dropouts. The datasets obtained under these two imputation scenarios were analyzed with the mixed regression models, as obtained after backward deletion of nonsignificant (interaction) effects in the analysis of only the complete cases.

\section{Results}

\section{Sample characteristics}

Figure 2 shows the flow of PNs included in the trial from initial assessment of eligibility to randomization and completion of baseline and follow-up questionnaires. Of the 346 PNs assessed for eligibility, 49 (14.2\%) did not meet inclusion criteria, and 18 (5.2\%) refrained from participation. After randomization, 147 (49.5\%, 147/297) and 122 (41.1\%, 122/297) PNs were allocated to the intervention and control group, respectively, and completed the baseline questionnaire. Unequal group sizes are the result of chance, as randomization took place at respondent level, and each PN had a 50\% probability of being allocated to either group.

The baseline sample of PNs (Table 1 ) had a mean age of 47.3 years (range: 23-66), the vast majority was female $(97.8 \%, 263 / 269)$, and very few PNs $(1.1 \%, 3 / 269)$ were current smokers. Nearly half $(47.2 \%, 127 / 269)$ worked in more than one general practice, and PNs worked on average almost 26 hours a week (range: 3-42). Many PNs (66.9\%, 180/269) were listed in the Dutch Stop Smoking Quality Register, and the mean-reported PN counseling experience was 5.6 years (range: 0-20). Finally, almost half of the PNs 
$(47.6 \%, 128 / 269)$ worked in a general practice with designated smoking cessation consulting hours, and nearly all $(92.6 \%, 249 / 269)$ reported to systematically register their patients' smoking status in their patient files. During the trial, PNs engaged in smoking cessation counseling with 5.6 different patients on average (range: 1-26).

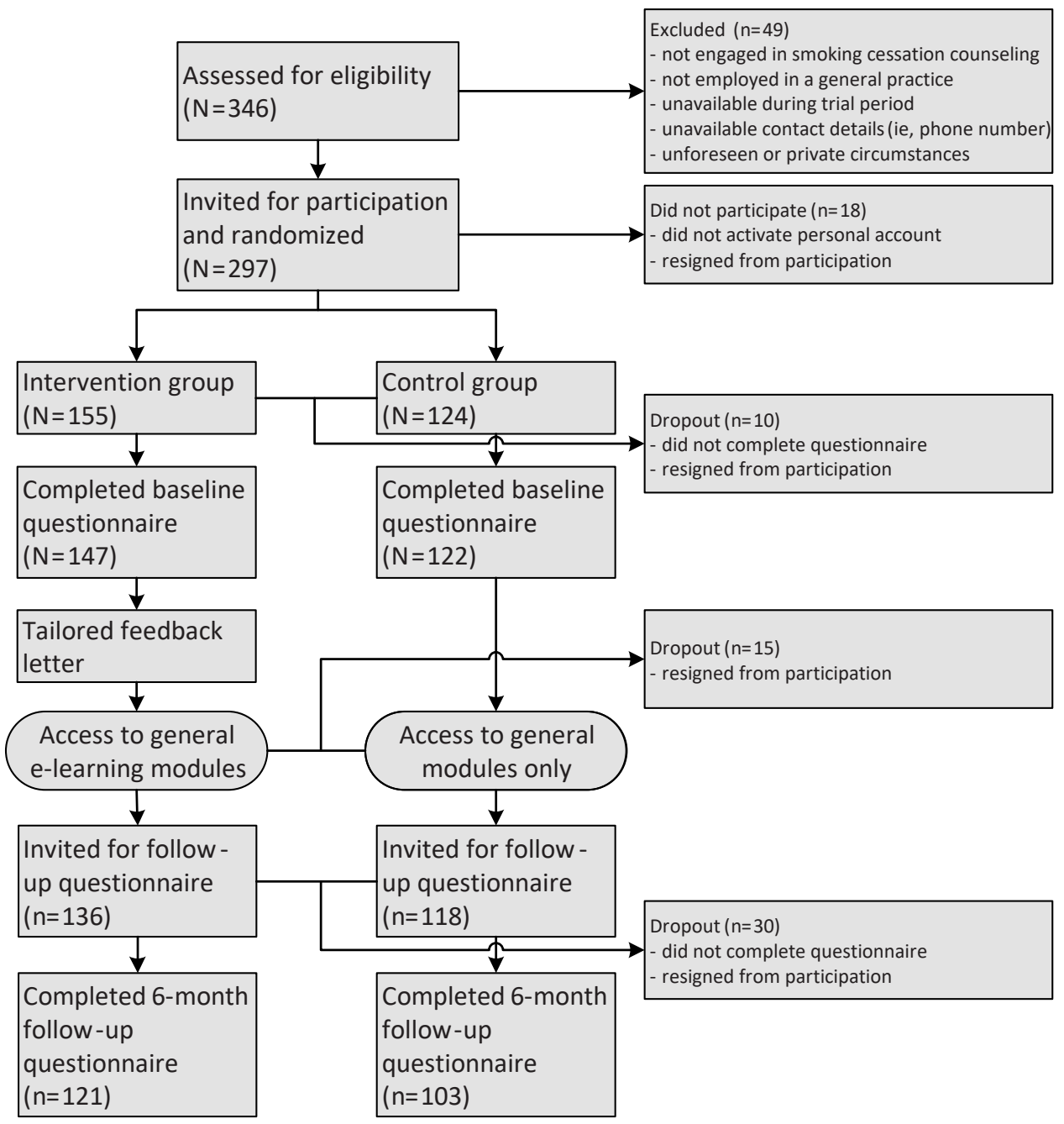

Figure 2 Flow and randomization of practice nurses that were recruited between January-June 2016

Baseline characteristics of PNs were comparable between intervention and control group, except for the presence of designated smoking cessation consulting hours, which was significantly more often reported by PNs in the control group $\left(\chi^{2}{ }_{1}=10.1 ; P=.001\right)$. Therefore, the presence of designated consulting-hours was included as a covariate in all effect analyses. 


\section{Chapter 5}

Table 1 Characteristics of practice nurses (PNs) and comparison of characteristics between intervention and control groups.

\begin{tabular}{|c|c|c|c|c|c|c|}
\hline Characteristics & $\begin{array}{l}\text { Overall } \\
\text { sample } \\
(\mathrm{N}=269)\end{array}$ & $\begin{array}{l}\text { Intervention } \\
\text { group } \\
(n=147)\end{array}$ & $\begin{array}{l}\text { Control } \\
\text { group } \\
(n=122)\end{array}$ & Chi-square & $\begin{array}{l}\mathrm{T} \text { (degrees of } \\
\text { freedom) }\end{array}$ & $P$ value \\
\hline Age in years, mean (SD) & $47.3(9.5)$ & $48.0(9.6)$ & $46.5(9.4)$ & N/A & $-1.345(267)$ & .18 \\
\hline Female, n (\%) & $263(97.8)$ & $143(97.3)$ & $120(98.4)$ & $X^{2}{ }_{1}=0.4$ & $\mathrm{~N} / \mathrm{A}$ & .55 \\
\hline Smoking status, n (\%) & & & & $X^{2}{ }_{2}=0.4$ & $\mathrm{~N} / \mathrm{A}$ & .84 \\
\hline Nonsmoker & $151(56.1)$ & $84(57.1)$ & $67(54.9)$ & & & \\
\hline Ex-smoker & $115(42.8)$ & $61(41.5)$ & $54(44.3)$ & & & \\
\hline Smoker & $3(1.1)$ & $2(1.4)$ & $1(0.8)$ & & & \\
\hline $\begin{array}{l}\text { Employed in }>1 \text { practice, } \\
n(\%)\end{array}$ & $127(47.2)$ & $70(47.6)$ & $57(46.7)$ & $X^{2}{ }_{1}=0.0$ & N/A & .88 \\
\hline $\begin{array}{l}\text { Working hours, mean } \\
\text { (SD) }\end{array}$ & $25.7(7.4)$ & $25.7(7.5)$ & $25.7(7.4)$ & $\mathrm{N} / \mathrm{A}$ & $-0.007(267)$ & .99 \\
\hline $\begin{array}{l}\text { Registration in Stop } \\
\text { Smoking Quality } \\
\text { Register, n (\%) }\end{array}$ & $180(66.9)$ & $95(64.6)$ & $85(69.7)$ & $\mathrm{X}^{2}{ }_{1}=0.8$ & $\mathrm{~N} / \mathrm{A}$ & .38 \\
\hline $\begin{array}{l}\text { Counseling experience in } \\
\text { years, mean (SD) }\end{array}$ & $5.6(3.7)$ & $5.5(3.6)$ & $5.8(3.8)$ & N/A & $-0.602(267)$ & .55 \\
\hline $\begin{array}{l}\text { Consulting hours } \\
\text { present, n (\%) }\end{array}$ & $128(47.6)$ & $57(38.8)$ & $71(58.2)$ & $X^{2}{ }_{1}=10.1$ & N/A & .001 \\
\hline $\begin{array}{l}\text { Registration of smoking } \\
\text { status in patient files, } \mathrm{n} \\
(\%)\end{array}$ & $249(92.6)$ & $135(91.8)$ & $114(93.4)$ & $X^{2}{ }_{1}=0.3$ & $\mathrm{~N} / \mathrm{A}$ & .62 \\
\hline $\begin{array}{l}\text { Number of counseled } \\
\text { patients during the trial, } \\
\text { mean (SD) }\end{array}$ & $5.6(4.4)$ & $5.8(4.1)$ & $5.3(4.8)$ & $\mathrm{N} / \mathrm{A}$ & $-0.700(209)$ & .49 \\
\hline
\end{tabular}

\section{Attrition analyses}

After 6 months, 254 PNs remained in the trial and were invited for the follow-up measurement, which was completed by $88.9 \%$ (121/136) of intervention group and $87.3 \%$ $(103 / 118)$ of control group PNs, respectively (Figure 2). Attrition analyses revealed that PNs who completed the follow-up measurement had a higher baseline intention to use the STIMEDIC guideline (odds ratio, OR=1.41; 95\% Cl 1.00-1.98) and had more baseline knowledge about the STIMEDIC guideline content (OR=1.39; 95\% Cl 1.07-1.82) compared with PNs who dropped out before the follow-up measurement (Table 2). Therefore, these two variables were also included as covariates in all effect analyses. 
Table 2 Baseline characteristics of practice nurses (PNs) and their odds to predict PN retention.

\begin{tabular}{lllll}
\hline Characteristics & Mean (SD) & Range & Odds ratio (95\% CI) & $P$ value \\
\hline Working hours & $25.7(7.4)$ & $3-42$ & $0.95(0.91-1.00)$ & .06 \\
Counseling experience in years & $5.6(3.7)$ & $0-20$ & $0.96(0.88-1.06)$ & .42 \\
Intention to use any evidence-based guideline (1-7) & $6.3(0.8)$ & $4-7$ & $0.86(0.51-1.45)$ & .57 \\
Intention to use STIMEDIC (1-7) & $5.5(1.1)$ & $2-7$ & $1.41(1.00-1.98)$ & .049 \\
STIMEDIC knowledge (0-18) & $14.6(1.3)$ & $11-18$ & $1.39(1.07-1.82)$ & .02 \\
Perceived advantages (1-5) & $4.2(0.6)$ & $2-5$ & $0.80(0.41-1.56)$ & .52 \\
Perceived disadvantages (1-5) & $1.9(0.6)$ & $1-4$ & $0.56(0.28-1.13)$ & .10 \\
Self-efficacy (1-5) & $2.8(0.6)$ & $1.3-5$ & $0.62(0.34-1.13)$ & .12 \\
Social modeling (1-5) & $3.2(0.9)$ & $1-5$ & $0.70(0.38-1.29)$ & .25 \\
Social support (1-5) & $3.3(0.8)$ & $1-5$ & $1.61(0.60-4.28)$ & .35 \\
Social norms (1-5) & $1.4(0.7)$ & $1.4-5$ & $1.34(0.47-3.83)$ & .58 \\
Action planning (0-8) & $6.4(1.7)$ & $0-8$ & $0.97(0.78-1.22)$ & .82 \\
Coping planning (0-10) & $6.7(2.7)$ & $0-10$ & $1.01(0.89-1.16)$ & .86 \\
Baseline guideline adherence (0-9) & $8.5(1.6)$ & $0-10$ & $1.06(0.86-1.31)$ & .59 \\
\hline
\end{tabular}

\section{Effect analyses}

\section{Overall adherence}

Table 3 shows the mixed regression results on PNs' overall guideline adherence; meancentered values are reported for variables included in interaction effects to enable meaningful interpretation of the effect of group (e.g. in Table 3, the effect of group allocation illustrates the effect of the intervention for PNs that score average on counseling experience). The results reveal a significant interaction effect of group allocation with counseling experience $(P=.045)$ and a main effect of perceived advantages of guideline use $(P=.03)$. Subgroup analyses showed that for PNs with more than average counseling experience (i.e. mean $+1 \mathrm{SD}=9.4$ years of experience), allocation to the intervention group (i.e. access to the $\mathrm{CT}$ e-learning program) resulted in a significantly higher overall adherence compared with the control group (beta=.589; 95\% Cl 0.111-1.068; $P_{\text {Holm-Bonfer- }}$ roni=.048). The subgroup analysis for less experienced PNs (i.e. mean $=5.6$ years of experience and mean $-1 \mathrm{SD}=1.9$ years of experience) revealed no significant intervention effect.

Table 3 Results of backward linear mixed regression analysis on practice nurses' (PNs') overall guideline adherence.

\begin{tabular}{lllc}
\hline Final model & Coefficient & $95 \% \mathrm{Cl}$ & $P$ value \\
\hline Group (control=0; intervention=1) & .245 & -0.087 to 0.577 & .19 \\
Counseling experience & -.046 & -0.113 to 0.021 & .18 \\
Perceived advantages & .319 & $0.031-0.608$ & .03 \\
Group* counseling experience & .092 & $0.002-0.183$ & .045 \\
\hline
\end{tabular}




\section{Step-based adherence}

Multimedia Appendix 1 shows the results on PNs' step-based adherence for each step separately; again mean-centered values are reported to enable meaningful interpretation of the effect of group in the presence of a significant interaction. Regarding adherence to step 1 (i.e. advising to quit smoking), a significant main effect was found of PNs' baseline adherence to step $1(P=.002)$. Regarding adherence to step 2 (i.e. assessing smoking profile and smoking history), a significant interaction effect of group allocation with perceived advantages of guideline use $(P=.001)$ was identified. Regarding adherence to step 3 (i.e. assessing motivation to quit), an interaction effect with self-efficacy $(P=.02)$ was found. Regarding adherence to step 4 (i.e. increasing motivation), a borderline significant interaction effect of group allocation with counseling experience $(P=.06)$ was found, as well as main effects of baseline adherence to step $4(P=.01)$ and of perceived advantages of guideline use $(P=.03)$. Regarding adherence to step 5 (i.e. assessing barriers to quitting), significant interaction effects of group allocation with social modeling $(P=.009)$ and with social support were found $(P=.046)$. Regarding adherence to step 6 (i.e. discussing barriers), a significant main effect was found of perceived advantages of guideline use $(P=.045)$. Regarding adherence to step 7 (i.e. informing about cessation aids), significant interaction effects of group allocation with perceived disadvantages $(P=.01)$ and with self-efficacy $(P=.001)$ and a borderline significant interaction effect with counseling experience $(P=.05)$ were found. Furthermore, a significant main effect of social modeling ( $P=.01$ ) was found. Regarding adherence to step 8 (i.e. making a quit plan and setting a quit date), significant interaction effects of group allocation with counseling experience $(P=.01)$, with social support $(P=.001)$, and with social norms $(P=.005)$ were found. Concerning adherence to step 9 (i.e. arranging follow-up after the quit date), an interaction with social support $(P=.04)$ was found.

Detailed results on subgroup analyses for each significant interaction effect are presented in Multimedia Appendix 2. Overall, the results show that higher adherence scores on individual guideline steps of intervention group PNs, compared with control group PNs, occur for (1) high levels of counseling experience and perceived social support and (2) low levels of perceived advantages, perceived disadvantages, self-efficacy and social modeling, and little social norms.

\section{Sensitivity analyses}

After replicating the final mixed regression models following an optimistic imputation scenario, results for overall guideline adherence indicated that only the main effect of perceived advantages remained significant. Regarding step-based adherence, similar results were found for adherence to guideline steps 1, 2, and 8. Concerning adherence to steps 5 and 7 only, the interaction effects with social modeling and self-efficacy could be replicated, and concerning adherence to step 4, only the main effect of perceived advantages remained significant. Main and interaction effects concerning guideline steps 3, 6, and 9 
were no longer or only marginally significant after conducting these optimistic sensitivity analyses.

After replicating the final mixed regression models following a pessimistic imputation scenario, main and interaction effects for overall adherence were no longer or only marginally significant. Regarding step-based adherence, similar results were found for adherence to guideline steps $1,6,8$, and 9, whereas regarding step 4, only main effects of baseline adherence to step 4 and perceived advantages remained significant. Main and interaction effects concerning guideline steps 2, 3, 5, and 7 were no longer or only marginally significant after conducting these pessimistic sensitivity analyses.

\section{Discussion}

\section{Principal findings}

The present RCT tested the effectiveness of a novel CT e-learning program for PNs to improve their adherence to evidence-based smoking cessation guidelines in Dutch general practice. Our results suggest that among PNs with more than average smoking cessation counseling experience, access to the $\mathrm{CT}$ e-learning program resulted in significantly better guideline adherence. A more detailed inspection of PNs' guideline adherence revealed comparable results for several specific guideline steps (i.e. increasing motivation, discussing cessation aids, and making a quit plan). Additionally, a trend was observed that for PNs reporting less favorable baseline levels for behavioral predictors such as self-efficacy and favorable levels of perceived social support, the e-learning program was effective in improving guideline adherence.

Regarding PNs' counseling experience, subgroup analyses illustrated that more experienced PNs in the intervention group adhered significantly better to the guideline (i.e. difference of adherence to 0.6 steps) at follow-up compared with equally experienced PNs in the control group. This finding means that the CT e-learning program successfully promoted guideline adherence among PNs with more counseling experience, which is in line with the fact that a significant positive association between counseling experience and PNs' baseline guideline adherence was found (Multimedia Appendix 3). Experienced PNs might have been better able to practically apply the content of the e-learning program during their counseling, as they have likely dealt with many different types of smokers and difficult counseling situations in the past. Due to these past coping experiences, it could have been easier for them, compared with less experienced PNs, to translate the theoretically grounded e-learning content to counseling situations encountered in practice, leading to improved guideline adherence. Yet, evidence about the influence of HCPs' counseling experience on their application of evidence-based guidelines is scarce. One study among physicians investigated the association between work experience and guideline adherence after taking an e-learning course but could not establish such an 
association (Nicastro et al., 2015). Another study focused on the relation between work experience and knowledge and found that more experienced professionals scored higher on knowledge after using a nontailored interactive video intervention (Bond et al., 2017). However, an association of experience with knowledge was not established among PNs in the present trial (Multimedia Appendix 3), and neither was it found in a study about the effects of an e-learning course on the knowledge level of nursing staff (van de Steeg et al., 2015). Hence, an alternative explanation could be considered; perhaps experienced PNs were able to spend their counseling time more efficiently, giving them opportunity to visit the CT e-learning program more often (e.g. in between consultations) and-as a consequence-benefit more from its content. However, although more frequent users were more adherent in the present trial, program usage (mean number of module visits=3.2, SD=6.2, range: $0-48$ ) was not significantly associated with PNs' guideline adherence, nor did we identify that more experienced PNs were more frequent users (data not shown). This is comparable with a study among GPs, in which factors such as working experience were not predictive of usage of an e-learning program to promote dementia guideline adherence (Waldorff, Steenstrup, Nielsen, Rubak, \& Bro, 2008). It is important to further investigate if and why HCPs, including PNs, with more counseling experience benefit more from CT e-learning programs. For example, process evaluation of users' interaction with a program can aid our understanding of the working mechanisms (e.g. targeted behavioral predictors) of exposure to tailored program content (Jager et al., 2016). Such insights could subsequently be used to improve a program's effectiveness for individuals with varying levels of counseling experience (e.g. by additionally tailoring program content on an individual's level of experience).

Results regarding PNs' step-based guideline adherence illustrated that, besides counseling experience, several behavioral predictors could explain better adherence scores of PNs in the intervention group compared with the control group. PNs' perceived advantages and disadvantages of guideline application, level of self-efficacy, social modeling, social norms, and social support at baseline all moderated the e-learning program's effect on PNs' step-based adherence. Although moderation effects could not be replicated precisely for each separate guideline step, a trend was observed that especially a high level of baseline social support and lower baseline levels of the other predictors were related to better effects of the program on adherence. As the content of the e-learning program was designed to especially target such behavioral predictors of guideline adherence (de Ruijter et al., 2016), it is likely that intervention group PNs, initially scoring less favorable on these predictors, were more able to significantly improve their adherence through progress made on these behavioral predictors. An analysis of PNs' change scores concerning these behavioral predictors, indeed found that intervention group PNs had more favorable scores compared with control group PNs, indicating more progress in terms of their levels of perceived advantages, disadvantages, self-efficacy, social modeling, social norms, and social support (data not shown). Although these change scores 
were not statistically significant, it is likely that the small improvements on all these predictors together contributed to better step-based adherence scores among intervention group PNs (de Vries, 2017).

\section{Strengths and limitations}

A strength of the present trial is that we enrolled a substantial number of PNs and managed to retain $83.3 \%$ (224/269) of them at 6-month follow-up. In comparison, in other studies among nurses, retention rates were considerably lower, e.g. studies among hospital nurses that reported 3-month and 6-month follow-up retention rates of 68\% and $58 \%$ (Sarna, Bialous, Wells, \& Brook, 2017), or 56\% and 48\%, respectively (Ketelaar et al., 2014). A high retention rate is essential to obtain adequate power for conducting statistical analyses. Moreover, the PNs in the present trial were able to counsel many smokers during the intervention period, resulting in a large amount of data collected on individual smoking cessation trajectories $(\mathrm{N}=1175)$. As each individual PN used the counseling checklist to self-report their application of guideline steps during each consultation with these smokers, we obtained data on PNs' guideline adherence from at least one consultation per smoker and from on average six different smokers' counseling trajectories per PN. Our primary outcome measure therefore reflects PNs' guideline adherence during the entire 12-month trial period instead of at a single time point only (e.g. at the end of the intervention period). Outcome measures composed of multiple measurement points are expected to provide a more reliable insight in the target behavior compared with a single measurement point (Murphy \& Davidshofer, 2005). A final strength is the fact that PNs' guideline adherence was assessed and analyzed from a step-based perspective, yielding more detailed insights than only taking overall guideline adherence into account.

Nevertheless, we also experienced some challenges during the trial. Our intention was to triangulate data on PNs' guideline adherence from both smokers' and PNs' perspectives to compute the primary outcome measure (de Ruijter et al., 2016). Unfortunately, only 33.3\% of smokers counseled by PNs (391/1175) also participated in the trial themselves, i.e. completed the baseline smoker questionnaire. Qualitative posttrial interviews with PNs $(\mathrm{N}=17)$ revealed issues such as time constraints of both smokers and PNs (i.e. trial participation was not addressed during the consultation) and smokers' privacy concerns as reasons for the low participation rate among smokers (data not shown). As a result, conducting effect analyses with these data would be unreliable because of substantial loss of power and selective inclusion of smokers. Moreover, a comparison of smoker-reported data from baseline questionnaires with PN-reported data from counseling checklists revealed significantly higher adherence scores reported by smokers $\left(t_{390}=-6.73, P<.001\right)$. As we observed possible ceiling effects in these adherence scores, smoker-reported data were deemed to be unreliable for being used as outcome measure. An explanation could be that smokers overestimated their PNs' guideline adherence, as they were recruited for participation in the trial by their PN, who also supported them 
with smoking cessation. Perhaps they were afraid that providing critical answers to questions about their PNs' performance could influence the relationship with their PN. Although smokers were informed that data were treated anonymously and were not reported back to PNs, the phenomenon of social desirability is often observed when collecting data from patients in a health care setting (Edwards, Staniszweska, \& Crichton, 2004). It, hence, seems that collecting patient data might not always be reliable and that more objective data collection methods are required. A second limitation was that 58 PNs $(21.6 \%, 58 / 269)$ did not manage to recruit and counsel smokers during the intervention period, resulting in lacking data on these PNs' guideline adherence during consultations. As a result, these PNs could not be included in the effect analyses. Nevertheless, inclusion of data from the remaining PNs $(\mathrm{N}=211)$ still ensured adequate power (alpha=5\%; beta $=10 \%$ ) to conduct mixed regression analyses concerning their guideline adherence (de Ruijter et al., 2016). To examine the sensitivity of these results, the final regression models were repeated following an optimistic and pessimistic imputation scenario. This sensitivity analysis showed that results could have been sensitive to informative dropout (i.e. missingness related to unobserved variables), as some main and interaction effects disappeared in both scenarios (Schafer \& Graham, 2002). Yet, most results were similar to the results of the complete-case analyses, indicating their robustness.

\section{Implications}

In light of the issues described, it would be worthwhile to investigate additional methods to gather data on PNs' guideline adherence. One such method could be qualitative data collection, by conducting content analyses of audio or video recordings of smoker-PN consultations (Brandes \& van Weert, 2017; Henry, Penner, \& Eggly, 2017; Lorencatto, West, Christopherson, \& Michie, 2013). In the present trial, however, PNs were very reluctant in agreeing to make an audio recording of a smoking cessation consultation, resulting in one or more successful recordings for only $11.5 \%$ of PNs (31/269) and 42 recordings in total. During posttrial interviews, PNs reported barriers such as privacy concerns (of both PN and smoker) and the perception that making a recording would influence the interaction with the smoker during a consultation. As a consequence, PNs believed that the recordings would not provide reliable insight into their counseling approach; a phenomenon also described in other recent studies (Moloczij et al., 2017; van Bruinessen, Leegwater, \& van Dulmen, 2017). Another potential method to collect data on PNs' guideline adherence could be to ask PNs to respond to simulated practice situations or clinical vignettes (i.e. case studies of smokers visiting their practice for cessation support) to assess their application of evidence-based guideline steps. Earlier studies applied this method, for instance, in written format concerning physiotherapists' guideline adherence (Rutten et al., 2010) or video-based format concerning suicide guideline implementation (de Beurs et al., 2015). Similarly, PNs could be provided with clinical vignettes that describe different types of smokers in terms of (1) Motivation status (e.g. 
unmotivated, contemplating, and motivated to quit); (2) Perceived barriers toward quitting (e.g. smoking partner, weight gain, and stress); and (3) Requests for pharmacotherapy (e.g. desire for particular medicine, refusing any pharmacotherapy, and favoring alternative medicine), to assess their guideline adherence in various situations that they could encounter in practice. Research with such clinical vignettes among PNs is needed to determine the potential value of using vignettes to reliably measure PNs' smoking cessation guideline adherence.

Furthermore, the results of the present trial illustrated that the CT e-learning program successfully improved smoking cessation guideline adherence of experienced PNs. Unfortunately, it was also established that usage of the program by PNs in the trial was quite limited (i.e. three module visits on average). This means that the program's effectiveness could potentially be increased when implementation of the program by PNs (i.e. number of modules visits) improves. During posttrial interviews, PNs mentioned that receiving a reminder to visit the program more often could be a potential strategy to stimulate program usage. Furthermore, a process evaluation could inform improving alterations to the program's tailored content to extend the program's effectiveness beyond experienced PNs. When such strategies would be combined with program implementation among a larger population of PNs, this could further substantiate the impact of the CT e-learning program on the quality of smoking cessation care in the Netherlands.

\section{Conclusions}

Providing PNs access to a novel CT e-learning program resulted in significantly better adherence to evidence-based smoking cessation guidelines among Dutch PNs experienced in smoking cessation counseling, compared with similar PNs without access to this program. More favorable improvements on behavioral predictors of guideline adherence among intervention group PNs may explain better adherence scores. To further substantiate the effectiveness of e-learning programs on guideline adherence by HCPs, alternative methods of collecting data on guideline adherence should be explored, and strategies are needed to promote program usage and to also support less experienced PNs to adhere to evidence-based smoking cessation guidelines. This could subsequently inform widespread implementation of the e-learning program among PNs. 


\section{Chapter 5}

Multimedia appendix 1: Results of backward logistic mixed regression analyses on practice nurses' (PNs') stepbased guideline adherence

\begin{tabular}{|c|c|c|}
\hline Final model & OR $(95 \% \mathrm{Cl})$ & $P$ value \\
\hline \multicolumn{3}{|l|}{ Step 1} \\
\hline Group (control=0; intervention=1) & $1.164(0.705-1.920)$ & .55 \\
\hline Baseline adherence step 1 & $1.199(1.068-1.345)$ & .002 \\
\hline \multicolumn{3}{|l|}{ Step 2} \\
\hline Group (control=0; intervention=1) & $1.004(0.652-1.545)$ & .99 \\
\hline Perceived advantages & $2.658(1.593-4.437)$ & .000 \\
\hline Group* perceived advantages & $0.298(0.143-0.621)$ & .001 \\
\hline \multicolumn{3}{|l|}{ Step 3} \\
\hline Group (control =0; intervention=1) & $2.042(1.065-3.918)$ & .03 \\
\hline Self-efficacy & $1.597(0.813-3.139)$ & .17 \\
\hline Group * self-efficacy & $0.288(0.097-0.851)$ & .02 \\
\hline \multicolumn{3}{|l|}{ Step 4} \\
\hline Group (control=0; intervention=1) & $1.347(0.846-2.144)$ & .21 \\
\hline Counseling experience & $0.946(0.863-1.037)$ & .24 \\
\hline Baseline adherence step 4 & $1.148(1.032-1.277)$ & .01 \\
\hline Perceived advantages & $1.548(1.038-2.308)$ & .03 \\
\hline Group * counseling experience & $1.135(0.995-1.293)$ & .06 \\
\hline \multicolumn{3}{|l|}{ Step 5} \\
\hline Group (control=0; intervention=1) & $1.294(0.717-2.336)$ & .39 \\
\hline Intention to use STIMEDIC & $1.289(0.985-1.687)$ & .06 \\
\hline Social modeling & $2.058(0.995-4.255)$ & .05 \\
\hline Social support & $0.571(0.247-1.320)$ & .19 \\
\hline Group * social modeling & $0.259(0.095-0.710)$ & .009 \\
\hline Group * social support & $3.094(1.019-9.387)$ & .046 \\
\hline \multicolumn{3}{|l|}{ Step 6} \\
\hline Group (control=0; intervention=1) & $1.175(0.710-1.947)$ & .53 \\
\hline Perceived advantages & $1.550(1.010-2.377)$ & .045 \\
\hline \multicolumn{3}{|l|}{ Step 7} \\
\hline Group (control=0; intervention=1) & $0.854(0.435-1.675)$ & .65 \\
\hline Counseling experience & $0.891(0.891-1.001)$ & .05 \\
\hline Perceived disadvantages & $2.162(0.792-5.900)$ & .13 \\
\hline Self-efficacy & $4.353(1.628-11.638)$ & .003 \\
\hline Social modeling & $1.654(1.118-2.446)$ & .01 \\
\hline Group * counseling experience & $1.184(0.997-1.406)$ & .05 \\
\hline Group * perceived disadvantages & $0.222(0.067-0.728)$ & .01 \\
\hline Group * self-efficacy & $0.109(0.030-0.403)$ & .001 \\
\hline
\end{tabular}


Multimedia appendix 1: Results of backward logistic mixed regression analyses on practice nurses' (PNs') stepbased guideline adherence

\begin{tabular}{llc}
\hline Final model & OR $(95 \% \mathrm{Cl})$ & $P$ value \\
\hline Step 8 & & .05 \\
Group (control =0; intervention=1) & $1.765(0.996-3.129)$ & .31 \\
Counseling experience & $0.949(0.858-1.049)$ & .02 \\
Social support & $0.314(0.121-0.814)$ & .07 \\
Social norms & $2.515(0.929-6.814)$ & .01 \\
Group * counseling experience & $1.215(1.048-1.409)$ & .001 \\
Group * social support & $10.713(2.753-41.697)$ & .005 \\
Group * social norms & $0.124(0.029-0.536)$ & .59 \\
Step 9 & & .02 \\
Group (control=0; intervention=1) & $1.121(0.735-1.711)$ & .04 \\
Social support & $0.600(0.391-0.920)$ & \\
Group * social support & $1.817(1.032-3.198)$ & \\
\hline
\end{tabular}




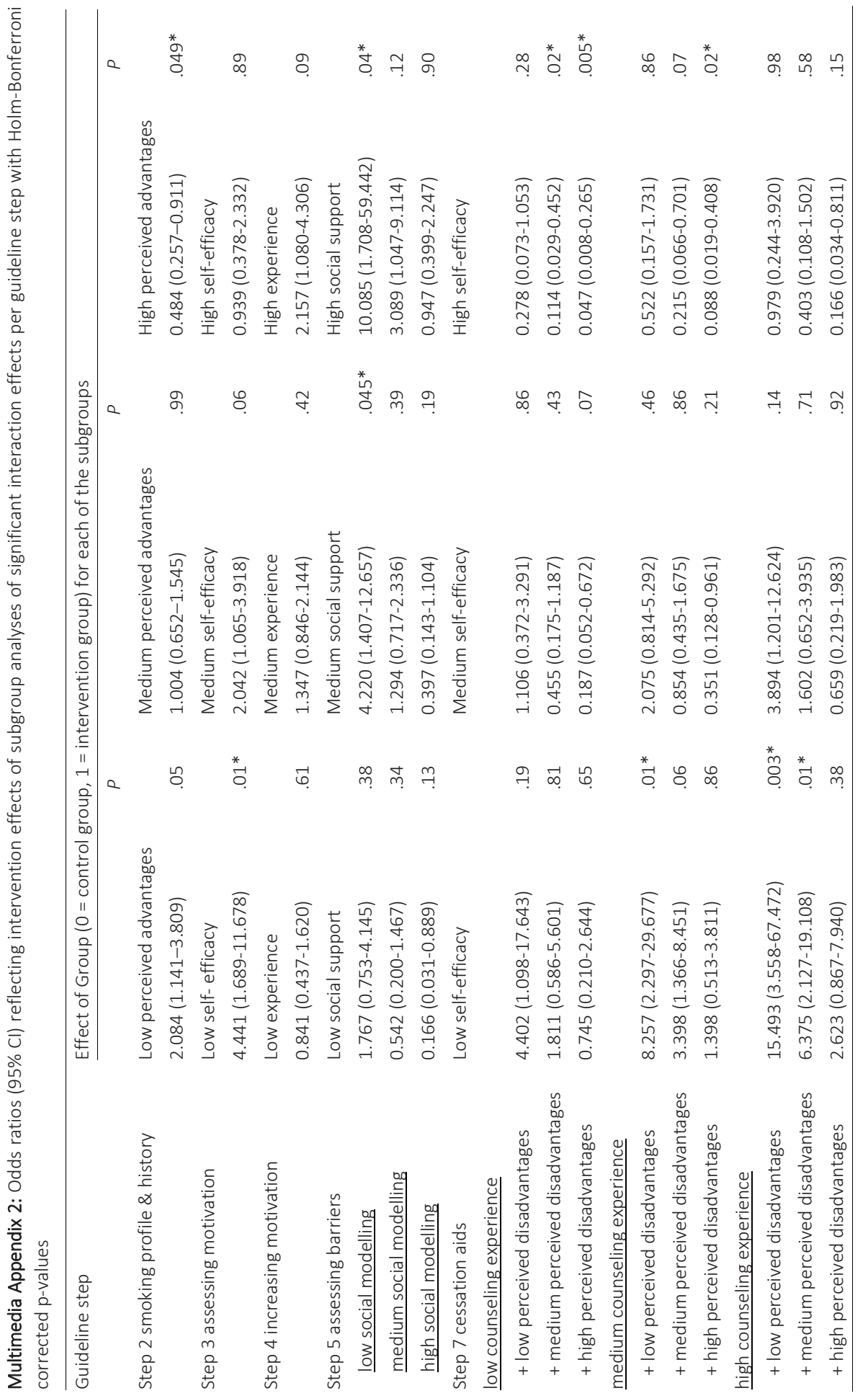




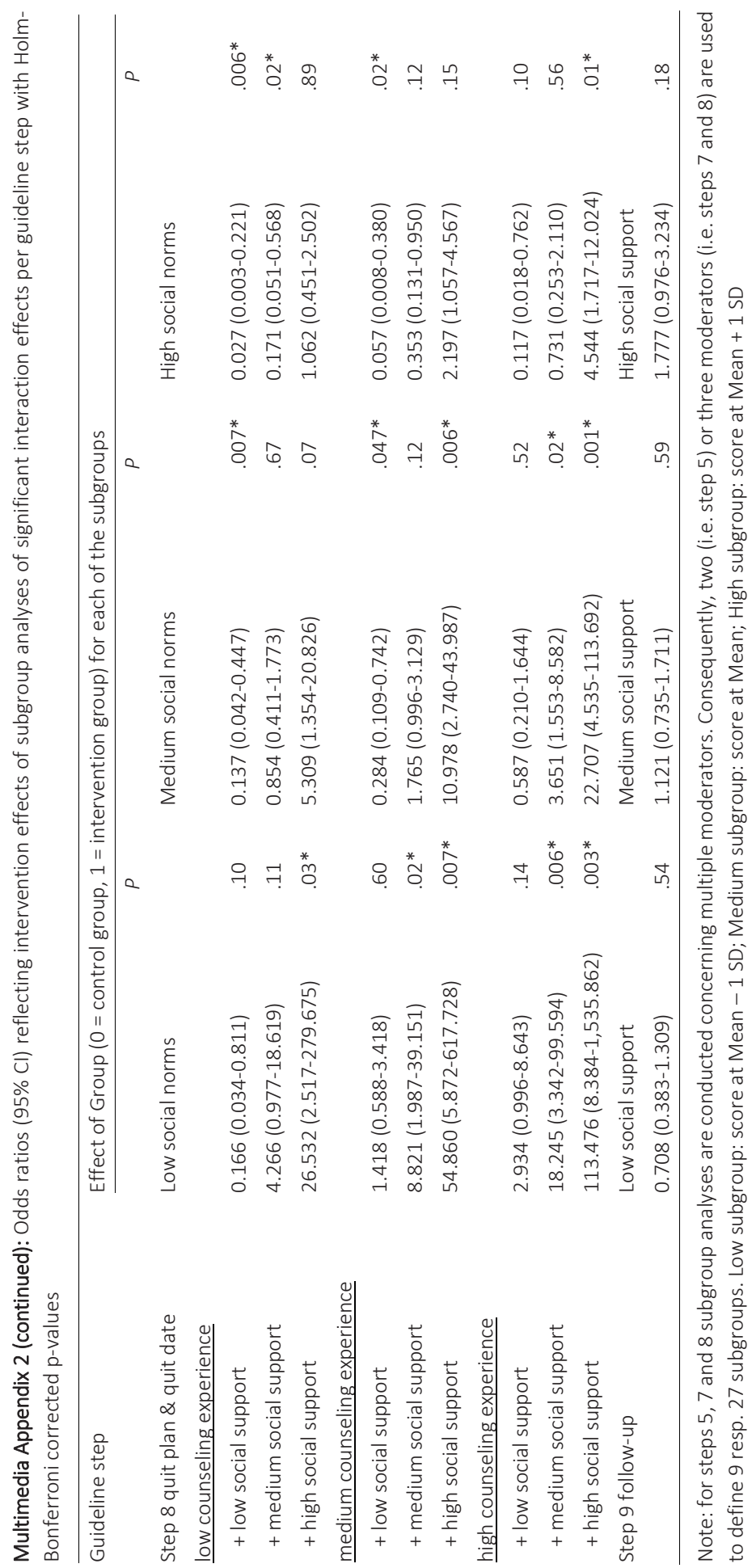


Chapter 5

Multimedia Appendix 3: Correlation matrix of practice nurse (PN) characteristics

\begin{tabular}{|c|c|c|c|c|c|c|c|c|c|c|c|c|c|}
\hline & 1 & 2 & 3 & 4 & 5 & 6 & 7 & 8 & 9 & 10 & 11 & 12 & 13 \\
\hline 1. Working hours & 1.00 & 0.06 & .07 & -.02 & -.03 & -.02 & -.03 & -.01 & .10 & -.06 & -.02 & -.00 & .10 \\
\hline 2. Counseling experience & & 1.00 & -.02 & .00 & .08 & -.01 & -.10 & .03 & -.02 & -.05 & -.07 & .02 & .16 \\
\hline 3. Designated consulting-hours & & & 1.00 & .01 & -.03 & .09 & .10 & -.03 & .07 & .13 & .09 & .07 & .10 \\
\hline $\begin{array}{l}\text { 4. Intention to use any evidence-based } \\
\text { guideline }\end{array}$ & & & & 1.00 & .41 & -.00 & .33 & -.39 & .12 & .31 & .29 & .31 & .12 \\
\hline 5. Intention to use STIMEDIC ${ }^{\circledR}$ & & & & & 1.00 & .07 & .23 & -.17 & .11 & .18 & .13 & .16 & .04 \\
\hline 6. STIMEDIC ${ }^{\circledR}$ knowledge & & & & & & 1.00 & .10 & -.08 & .01 & -.01 & .05 & .06 & -.05 \\
\hline 7. Perceived advantages & & & & & & & 1.00 & .40 & .11 & .29 & .34 & .40 & -.00 \\
\hline 8. Perceived disadvantages & & & & & & & & 1.00 & -.30 & -.30 & -.33 & -.33 & $3-.17$ \\
\hline 9. Self-efficacy & & & & & & & & & 1.00 & .16 & .17 & .17 & .14 \\
\hline 10. Social modelling & & & & & & & & & & 1.00 & .71 & .69 & .16 \\
\hline 11. Social support & & & & & & & & & & & 1.00 & .88 & .20 \\
\hline 12. Social norms & & & & & & & & & & & & 1.00 & 0.21 \\
\hline 13. Baseline guideline adherence & & & & & & & & & & & & & 1.00 \\
\hline
\end{tabular}

Note: Data in italics denote a significant correlation 
Chapter

An economic evaluation of a computertailored e-learning program to promote smoking cessation counseling guideline adherence among practice nurses 


\section{Abstract}

Objective: To conduct an economic evaluation of a tailored e-learning program, which successfully improved practice nurses' smoking cessation guideline adherence, from a societal perspective.

Methods: The economic evaluation was embedded in a randomized controlled trial, in which 269 practice nurses recruited 388 smoking patients. Cost-effectiveness was assessed using guideline adherence as effect measure on practice nurse level, and continued smoking abstinence on patient level. Cost-utility was assessed on patient level, using patients' Quality Adjusted Life Years (QALYs) as effect measure.

Results: The e-learning program was likely to be cost-effective on practice nurse level, as adherence to an additional guideline step cost $€ 1,586$. On patient level, cost-effectiveness was moderately likely after six months (cost per additional quitter: $€ 7,126)$, but not after twelve months. The cost-utility analysis revealed moderate cost-effectiveness (cost per QALY gained: €18,431) on patient level.

Conclusion: Providing practice nurses with a tailored e-learning program is cost-effective to improve their smoking cessation counseling. Indirect cost-effectiveness on patient level was not found, which may result from smoking relapse.

Practice implications: Widespread implementation of the e-learning program can improve the quality of smoking cessation care in Dutch general practice. Yet, strategies to prevent patients' smoking relapse should be further explored to improve patients' long-term abstinence. 


\section{Introduction}

Smoking cessation interventions in primary care, such as individual counseling by healthcare professionals (HCPs) in general practice, can effectively increase smokers' quit rates (Stead et al., 2016). In the Netherlands, $72 \%$ of smokers visited their general practice at least once in 2016, indicating the potential reach of smoking cessation interventions in this setting (Springvloet \& van Laar, 2017). Smoking cessation counseling in general practice is nowadays predominantly provided by trained practice nurses (PNs) (Freund et al., 2015), applying evidence-based counseling guidelines (Trimbos-instituut \& NHG, 2016). These highly educated nurses are employed in $88 \%$ of Dutch general practices and are involved in smoking cessation counseling as part of their responsibilities regarding chronic patient care and lifestyle counseling (Heiligers et al., 2012; van Hassel et al., 2016). PNs often take over these tasks from their general practitioner (GP). A systematic review demonstrated that PNs had equal or better outcomes regarding patient satisfaction and quality of care compared to GPs, against equal or less associated costs (Swan, Ferguson, Chang, Larson, \& Smaldone, 2015). Also in the Netherlands PN-led care proved to be comparable to GP-led care both in general (Dierick-van Daele et al., 2009) and concerning smoking cessation counseling specifically (van Rossem et al., 2017), illustrating the potential of PN-led care to be cost saving.

Thus, we know that PNs can reach many smokers in Dutch general practice (Springvloet \& van Laar, 2017) and that their smoking cessation counseling can potentially be cost saving (Dierick-van Daele et al., 2009; van Rossem et al., 2017). Nevertheless, previous studies showed that PNs do not systematically adhere to all smoking cessation counseling steps (de Ruijter, Smit, de Vries, Goossens, et al., 2017; de Ruijter, Smit, de Vries, \& Hoving, 2017; Whitehead et al., 2014). This means PN-led counseling is not adequately implemented in practice, which will lower the potential (cost-)effectiveness of their smoking cessation care (Glasgow et al., 2003). It is therefore important to support PNs to reach optimal guideline adherence, yet this requires both time and money as PNs need additional training to implement smoking cessation counseling according to the evidence-based guideline (Selby et al., 2015). Previously, studies testing counseling support interventions (i.e. web-based or face-to-face training) for HCPs have demonstrated that the impact of their smoking cessation counseling improved, as smokers more often received adequate and complete smoking cessation support from their HCP (Asfar et al., 2017; Unrod et al., 2007; Verbiest, Crone, et al., 2014). However, as such a counseling support intervention was not yet available for PNs, we developed a novel computer-tailored (CT) e-learning program for PNs to support them to optimally implement the national smoking cessation guideline (de Ruijter et al., 2016).

The content of this e-learning program was based on PNs' needs for individually relevant, easy-to-use web-based counseling support and incorporates PNs' preferences regarding program design and content (de Ruijter, Smit, de Vries, Goossens, et al., 2017). The program offers web-based counseling support in a relatively inexpensive and highly flexible way to a 
large group of PNs. The program's online availability ensures it can be consulted whenever PNs require and desire it. Program content is tailored to socio-cognitive factors, previously found to be associated with PN guideline adherence (de Ruijter et al., 2016), to create a personally relevant support tool for each PN. Subsequently, a randomized controlled trial (RCT) demonstrated that access to the e-learning program successfully improved guideline adherence among experienced PNs (de Ruijter, Candel, Smit, de Vries, \& Hoving, 2018). Yet, next to establishing the program's effectiveness, evidence regarding the cost-effectiveness is important to inform decision-making concerning care-related policy and resource allocation; this enables policy makers to compare costs and effects of different interventions with current practice and to subsequently make an informed decision on implementation of these interventions (Evers et al., 2010). Although some studies have conducted economic evaluations of face-to-face interventions to train HCPs in smoking cessation counseling (Cantor et al., 2015; Pinget et al., 2007), none have investigated the cost-effectiveness of web-based counseling support for HCPs.

Therefore, the aim of the present paper is to describe the results from a trial-based economic evaluation of the e-learning program from a societal perspective on two levels, the PN and patient level. On PN level, we will determine the cost-effectiveness of the elearning program to improve PNs' smoking cessation counseling. We hypothesize that improved counseling by PNs will positively affect quality of patient care. Hence on patient level, we will evaluate the cost-effectiveness and cost-utility of the e-learning program by also assessing indirect program effects on counseled patients' smoking cessation rates and quality of life.

\section{Methods}

\section{Study design}

This economic evaluation was embedded in an RCT testing the effectiveness of the e-learning program among Dutch PNs and their smoking patients with a twelve-month follow-up period. Evaluation by the Medical Ethics Committee Atrium-Orbis-Zuyd (14-N-17) revealed that no medical ethical clearance for this study was needed according to the rules of the Medical Research Involving Human Subjects Act (WMO). The study is registered with the Dutch Trial Register (NTR4436). A detailed description of the trial's design and the e-learning program's content is published elsewhere (de Ruijter et al., 2016).

\section{Participants and procedure}

\section{Practice nurses}

PNs enrolled in the RCT between January-June 2016. Eligible PNs were engaged in smoking cessation counseling in Dutch general practice, had Internet access and a working 
email account, and were sufficiently proficient in Dutch. After providing online informed consent and completing the baseline questionnaire, 269 PNs were randomized at respondent level to the intervention or control group of the trial through a computer software randomization device (de Ruijter et al., 2018).

\section{Smoking patients}

During the six-month trial, PNs were instructed to engage in counseling with smoking patients and recruit those patients for trial participation (Figure 1). Eligible patients were smokers of 18 years or older, had Internet access and a working email account, and were sufficiently proficient in Dutch. Interested patients could leave their email address with their PN and were subsequently invited via email to fill out the baseline questionnaire.

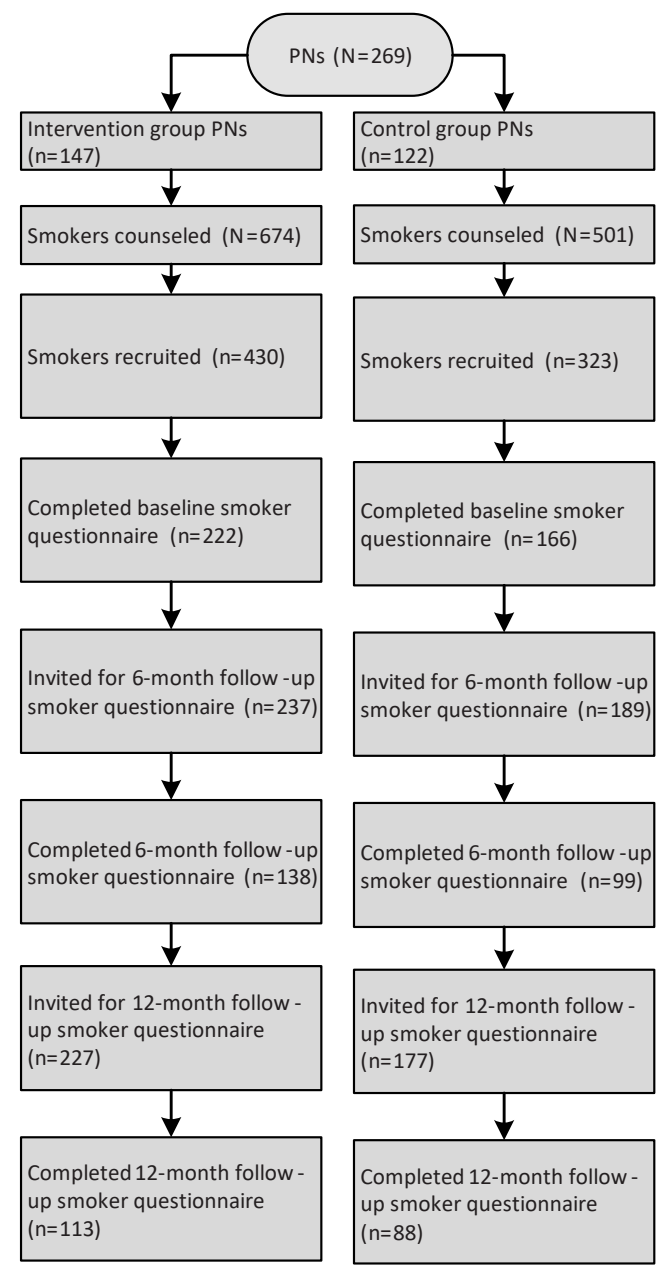

Figure 1 Flow of smoking patients counseled by PNs in the intervention and control group 
The e-learning program

The CT e-learning program consisted of 1) five e-learning modules with tailored advice, a forum, and smoking cessation counseling materials; and 2) three general modules containing project information, frequently asked questions about the trial, and a counseling checklist to self-report application of guideline steps. The content of the e-learning modules was based on a behavior change framework, the I-Change Model (de Vries, 2017), and previously developed, effective CT behavior change interventions (Bolman et al., 2015; Elfeddali et al., 2012; Smit et al., 2012; Stanczyk, Bolman, et al., 2014). Advice was tailored to individual PNs' questionnaire answers, addressing demographic characteristics (e.g. gender), pre-motivational factors (e.g. knowledge), motivational factors (e.g. self-efficacy), post-motivational factors (e.g. coping planning), intention (to use a smoking cessation guideline), and behavior (i.e. self-reported application of smoking cessation guideline steps). During the six-month trial, intervention group PNs ( $N=147)$ had full access to the e-learning program, whereas control group PNs only had access to the three general modules (de Ruijter et al., 2016).

\section{Economic evaluation}

This economic evaluation compared costs and effects concerning the CT e-learning program for PNs (i.e. intervention group with full program access) with costs and effects concerning regular $\mathrm{PN}$-led smoking cessation counseling (i.e. control group with access to general program modules only). The economic evaluation was conducted from a societal perspective, meaning that we also included costs and effects from outside the healthcare setting (Hakkaart-van Roijen, van der Linden, Bouwmans, Kanters, \& Tan, 2016). This study was applied on PN and patient level, and was based on national (Hakkaart-van Roijen et al., 2016) and international guidelines (Husereau et al., 2013), following five steps (Table 1): 1) identification of costs and effects, 2) measurement of costs and effects, 3) valuation of costs and effects, 4) calculation of a cost-effectiveness ratio, and 5) analysis of uncertainty (Evers et al., 2010).

\section{Identification of costs and effects}

Identification on PN level

On PN level, intervention and time costs were considered relevant. Intervention costs related to delivery of the e-learning program (e.g. hosting of the website and e-learning program). Program development costs and other research-related costs were excluded, as these would not be required for program implementation in practice. Time costs related to time lost due to program engagement and counseling smoking patients.

The primary outcome measure used in the cost-effectiveness analysis (CEA) was PNs' evidence-based smoking cessation guideline adherence. A change in quality of life was 
not expected among PNs, since PNs' counseling is rather related to their patients' health status. Hence, a cost-utility analysis (CUA) was not conducted on PN level.

\section{Identification on patient level}

On patient level, healthcare costs and patient costs were considered relevant. Healthcare costs related to patients' healthcare usage during the trial, including contact with HCPs, hospital admissions, prescribed and over-the-counter medication, and other types of care. Patient costs included travel costs and costs associated with time lost by participating in PN-led smoking cessation consultations.

The primary outcome measure was self-reported smoking abstinence for the CEA. For the CUA, the outcome measure was quality of life expressed in quality adjusted life years (QALYs) (de Ruijter et al., 2016).

Table 1 Five steps in conducting an economic evaluation (Evers et al., 2010)

1. Identification of All relevant costs and effects associated with the intervention and target behavior are costs and effects identified.

2. Measurement Costs are usually assessed via prospective cost diaries or retrospective cost questionnaires. of costs and effects Effects are often assessed via questionnaires. For measuring quality of life it is recommended to use instruments that measure generic rather than disease-specific quality of life, to promote comparability with interventions targeting other health behaviors.

3. Valuation of Costs are preferably valued according to reference prices listed in a country-specific costs and effects manual for conducting economic evaluations. Costs should be indexed to the same year to ensure prices are comparable. When valuing effects on quality of life it is required to calculate utility scores, representing preferences regarding a set of health states, which are used to calculate QALYs.

4. Calculation of a Costs and effects are combined into an ICER concerning behavior-specific effects, or an cost-effectiveness ICUR regarding QALYs. These ratios represent the difference in costs between intervention ratio and control group divided by the difference in effects between these groups. As both costs and effects of a new intervention are often higher, country-specific cut-off points have been established for the WTP per QALY gained, which vary according to disease severity.

5. Analysis of Sampling uncertainty can be dealt with by conducting bootstrap analyses, thereby drawing uncertainty multiple random samples (often thousands) from the original data set each with their own ICER/ICUR. These are used to estimate an intervention's probability to be preferred over the control treatment. Other types of uncertainty can be dealt with by conducting sensitivity analyses to test the robustness of the results under different assumptions.

$\mathrm{QALY}=$ quality adjusted life years; ICER=incremental cost-effectiveness ratio; ICUR=incremental cost-utility ratio; WTP=willingness to pay;

\section{Measurement of costs and effects}

\section{Measurement on PN level}

Costs for hosting the website (€58.35 for six months) and the CT e-learning program ( $€ 208$ for six months + a one-off user license of €2,350) were retrieved from providers. PNs' program engagement (i.e. number and duration of visits) was assessed through log 
data. PNs' time spent on counseling was assessed using the reported duration of each consultation via completed counseling checklists.

Guideline adherence was self-reported by PNs after every consultation with a smoking patient using the counseling checklist. Adherence ( $y / n)$ was assessed concerning the following guideline steps: 1) advising to quit smoking, 2) assessing smoking profile and history, 3) assessing quit motivation, 4) increasing motivation, 5) assessing barriers to quitting, 6) discussing barriers, 7) informing about cessation aids, 8) making a quit plan and setting a quit date, and 9) arranging follow-up after the quit date (de Ruijter et al., 2016).

\section{Measurement on patient level}

Healthcare costs were assessed using six-month retrospective online questionnaires, based on a previously developed questionnaire (Smit, Evers, et al., 2013), at six-month and twelve-month follow-up. Patients reported the number of contacts with HCPs: PN, GP, inpatient and/or outpatient specialist, mental HCP, occupational physician, lifestyle coach, social worker, pharmacist, and alternative medicine professional. Patients indicated the number and duration of hospital admissions, and reported the frequency and duration of other care (i.e. home-care, informal care, rehabilitation, physical therapy, dental care). Patients reported the dose, duration and frequency of prescribed smoking cessation medication (i.e. Varenicline, Bupropion, Nortriptyline, Paroxetine) and overthe-counter nicotine replacement therapy (i.e. nicotine patch, gum, spray) used, if any. Other types of medication (e.g. antibiotics, pain killers) were also reported, when used. Patients' travel and time costs were based on the number and duration of their PN-led consultations (i.e. number of checklists completed).

Smoking abstinence was assessed after six and twelve months by asking if patients had refrained from smoking ( $0=$ no; $1=y e s$ ) since baseline (i.e. continuous abstinence) (Mudde, Willemsen, Kremers, \& de Vries, 2006). Quality of life was assessed through the ICECAP questionnaire (Al-Janabi et al., 2012) to assess patients' quality of life as their general capability regarding five attributes: attachment, stability, autonomy, achievement and enjoyment (1=not capable; 2 =a little capable; 3=quite capable; 4=fully capable).

\section{Valuation of costs and effects}

Cost valuation was based on the guideline for conducting economic evaluation studies in healthcare (Hakkaart-van Roijen et al., 2016). Standardized costs prizes were used when available. Otherwise, costs were estimated based on real costs (e.g. average cost price based on real consultation prices of a HCP) or tariffs, while selecting the lowest price in case of uncertainty.

\section{Valuation on PN level}

Intervention costs were $€ 2,616.35$ (website $€ 58.35$, e-learning program $€ 2,558$ ). Program engagement was valued using productivity costs for paid work (€34.75/hour (Hakkaartvan Roijen et al., 2016)). Time costs concerning counseling were valued with cost prices 
for either a short (20 minutes or less; €9.07) or long consultation (over 20 minutes; $€ 18.33$ ) (Hakkaart-van Roijen et al., 2016), resulting in accumulated counseling costs per PN.

Guideline adherence was computed by combining all checklist data of the same smoker into a single score, between 0 (no step was adhered to) and 9 (all steps were adhered to). A mean adherence score was computed to summarize PNs' adherence across separate smokers counseled by the same PN.

\section{Valuation on patient level}

Healthcare costs were indexed to represent cost prices in 2016 (consumer price index=100.32). As reference prices in the guideline were from the year 2014 (consumer price index=99.40), cost prices were multiplied by 100.32/99.40 (price index 2016/price index 2014) (Centraal Bureau voor de Statistiek, 2017). The lowest dose and/or lowest price was chosen in case of uncertainty about the dose and/or price. For prescribed medication, first-use prescription charges (€14.50) were added to the cost price or repeateduse prescription charges (€7.25) when medication was also listed at another measurement. For prescribed medication the 'price per pill' was calculated, based on information from the Dutch database on medication costs (i.e. www.medicijnkosten.nl (Zorginstituut Nederland, 2017b)). In case of uncertainty about the duration of use, the standard treatment protocol was retrieved from the Dutch medication and pharmacotherapy database (i.e. www.farmacotherapeutischkompas.nl (Zorginstituut Nederland, 2017a)). Concerning over-the-counter (smoking cessation) medication, the 'price per pack' was calculated (Zorginstituut Nederland, 2017b).

To calculate travel costs, all consultations of each patient were multiplied with the average travel distance to a Dutch general practice (1.1 kilometers), parking fees (€3/visit) and transportation costs (€0.19/kilometer) (Hakkaart-van Roijen et al., 2016). The duration of consultations was valued using productivity costs for unpaid work ( $€ 14 /$ hour (Hakkaart-van Roijen et al., 2016)) to calculate time costs.

Smoking abstinence was valued to represent patients' continued abstinence ( $1=$ abstinent, $0=$ relapsed). ICECAP scores on each attribute were translated into a tariff score, resulting in an overall, accumulated utility value for capability, ranging from -0.001 (no capability) to 1 (full capability). For example, an ICECAP state of '44144' represents someone who is not capable to have autonomy but fully capable concerning the other attributes (i.e. utility score of 0.818) (Al-Janabi et al., 2012). QALYs were computed by taking the average of ICECAP utility scores at baseline and both follow-up measurements, representing a gain or loss in number of QALYs per year (Hakkaart-van Roijen et al., 2016). Gaining 0.9 QALYs either means gaining one year of near perfect capability or gaining 0.9 years of perfect capability. 
Calculation of a cost-effectiveness ratio

On PN level, all costs on PN and patient level, and PNs' guideline adherence were included to calculate the ICER, according to the formula: (costsintervention group - costscontrol gorup) / (guideline adherence $e_{\text {intervention group }- \text { guideline adherence }}$ control group). On patient level, also all costs were included. For the ICER, continued smoking abstinence after six or twelve months was included and for the ICUR, QALYs based on ICECAP utilities were included. To determine cost-effectiveness, the ICUR was compared to the Dutch willingness to pay (WTP) threshold, which is $€ 20,000$ per QALY for preventive interventions (Raad voor de Volksgezondheid en Zorg, 2006).

\section{Uncertainty analyses}

As cost distributions tend to be skewed, bootstrapping analyses (1,000 samples) were conducted to address uncertainty around estimated costs and to compute means and confidence intervals for costs. Bootstrapping (5,000 samples) was also conducted to address sample uncertainty when calculating an ICER/ICUR, resulting in the creation of costeffectiveness planes (CEP) and cost-effectiveness acceptability curves (CEAC). A CEP is a scatter plot expressing incremental costs on one axis and incremental outcomes on the other axis, showing the probability of the intervention being 1) cost-saving and more effective (dominant), 2) cost-saving and less effective, 3) cost-increasing and more effective, and 4) cost-increasing and less effective (dominated). The CEAC is used to explore the probability of the intervention being cost-effective for a range of WTP thresholds (Fenwick, O'Brien, \& Briggs, 2004).

Sensitivity analyses were conducted to test the robustness of the results. On PN level, the CEA was repeated while making a distinction between program users (i.e. at least one module visit) and non-users, instead of comparing intervention and control group PNs. On patient level, the CUA was repeated from a health-related quality of life perspective (i.e. based on EuroQol (Versteegh et al., 2016) utilities), instead of a capability perspective based on ICECAP utilities.

\section{Data analyses}

Missing data concerning costs and ICECAP scores were replaced by mean imputation, based on available data from the previous and next assessment. In case multiple assessments were missing, data were replaced using the last observation carried forward method. Data concerning smoking abstinence were analyzed according to the completecase principle. In secondary analyses, missing data on smoking abstinence were replaced using a conservative scenario, meaning that patient drop-outs were considered to be smoking.

Baseline comparability of PNs and patients was tested with Chi-square tests and independent-samples t-tests. These tests were also used to assess differences in effects between patients counseled by intervention and control group PNs after six and twelve 
months. On PN level, a difference in effect after twelve months was assessed with the independent-samples t-test.

Calculations of the ICER/ICUR and bootstrap analyses were conducted using Microsoft Office Excel 2011. Other analyses were conducted using SPSS 24.0.

\section{Results}

\section{Sample characteristics}

PNs' and patients' baseline characteristics are presented in Table 2 and Table 3, including a comparison of characteristics between the intervention and control group. No baseline differences were found between intervention and control group PNs, or between their counseled patients.

Table 2 Baseline characteristics of PNs in the intervention and control group

\begin{tabular}{llllll}
\hline Baseline PN characteristics & Total sample (N=269) & $\begin{array}{l}\text { Intervention } \\
\text { group (N=147) }\end{array}$ & $\begin{array}{l}\text { Control } \\
\text { group } \\
(\mathrm{N}=122)\end{array}$ & $\begin{array}{l}\text { Chi } \\
\text { square/ } \\
\text { t- } \\
\text { statistic }\end{array}$ \\
\hline Age in years, mean (SD) & $47.3(9.5)$ & $48.0(9.6)$ & $46.5(9.4)$ & -1.345 & .180 \\
Gender: female, (\%) & $263(97.8 \%)$ & $143(97.3 \%)$ & $120(98.4 \%)$ & .358 & .550 \\
Working hours, mean (SD) & $25.7(7.4)$ & $25.7(7.5)$ & $25.7(7.4)$ & -.007 & .994 \\
Counseling experience in years, mean (SD) & $5.6(3.7)$ & $5.5(3.6)$ & $5.8(3.8)$ & -.602 & .548 \\
Employed in > 1 practice (\%) & $127(47.2 \%)$ & $70(47.6 \%)$ & $57(46.7 \%)$ & .022 & .883 \\
\hline
\end{tabular}

Table 3 Baseline characteristics of patients counseled by intervention and control group PNs

\begin{tabular}{llllll}
\hline Baseline patient characteristics & $\begin{array}{l}\text { Total sample } \\
(\mathrm{N}=388)\end{array}$ & $\begin{array}{l}\text { Intervention } \\
\text { group }(\mathrm{N}=222)\end{array}$ & $\begin{array}{l}\text { Control group } \\
(\mathrm{N}=166)\end{array}$ & $\begin{array}{l}\text { Chi square/ } \\
\text { t-statistic }\end{array}$ \\
\hline Age in years, mean (SD) & $50.4(12.2)$ & $50.6(12.4)$ & $50.1(11.9)$ & -.410 & .68 \\
Gender: female (\%) & $226(58.2 \%)$ & $136(61.3 \%)$ & $90(54.2 \%)$ & 1.938 & .16 \\
SES (\%) & & & & .246 & .97 \\
- low & $14(3.6 \%)$ & $8(3.6 \%)$ & $6(3.6 \%)$ & & \\
- medium low & $118(30.4 \%)$ & $67(30.2 \%)$ & $51(30.7 \%)$ & & \\
- medium high & $136(35.1 \%)$ & $80(36.0 \%)$ & $56(33.7 \%)$ & & \\
- high & $120(30.9 \%)$ & $67(30.2 \%)$ & $53(31.9 \%)$ & & .72 \\
Quit intention (1-7), mean (SD) & $6.5(0.6)$ & $6.5(0.6)$ & $6.5(0.6)$ & -.358 & .81 \\
FTND (0-10), mean (SD) & $5.2(2.0)$ & $5.2(2.0)$ & $5.3(2.1)$ & .239 & .20 \\
ICECAP utility, mean (SD) & $0.85(0.1)$ & $0.85(0.1)$ & $0.84(0.1)$ & -1.295 & .47 \\
Healthcare costs in euros, mean (SD) & $430(1,358)$ & $474(1,580)$ & $372(987)$ & -.730 & .73 \\
\hline
\end{tabular}

${ }^{1}$ SES categories are based on a combination of an individual's level of education and the profession of the household's main breadwinner (Mudde et al., 2006)

2 FTND refers to the Fagerström test for nicotine dependence

${ }^{3}$ Costs during the prior six months 


\section{Annual costs and effects}

On PN level, intervention costs were higher in the intervention group ( $€ 57$ vs. $€ 6$ in the intervention control group) and time costs were comparable between the groups (Table 4). PNs' guideline adherence did not significantly differ between the intervention and control group.

On patient level, a significant difference was found regarding their score on the Fagerström test for nicotine dependence (FTND) at six-month follow-up; FTND scores were 3.1 $(S D=2.1)$ for intervention and $4.1(S D=2.4)$ for control group patients. No differences were found regarding patients' healthcare costs and patient costs, smoking abstinence, quit intention and ICECAP utility scores (Table 5). At 12-month follow-up, FTND scores no longer differed between intervention and control group patients. Differences in healthcare and patient costs, smoking abstinence, quit intention and ICECAP utility also remained non-significant (Table 5).

\section{Cost-effectiveness analyses}

On PN level, the CEA demonstrates that in comparison to the control group, €1,585.54 has to be paid in the intervention group for each additional guideline step that is on average adhered to by a PN. From a program use perspective (i.e. non-use vs. at least single use), the CEA shows that costs for adherence to each additional guideline step are $€ 3,588.57$ (Table 6). The CEAC shows that, concerning both perspectives, the program is already highly likely to be cost-effective at a WTP threshold of $€ 10,000$ (Appendix 1).

Table 4 Costs and effects on PN level until 12-month follow-up

\begin{tabular}{|c|c|c|c|c|}
\hline & Intervention group ( $N=117)$ & Control group ( $\mathrm{N}=94)$ & & \\
\hline \multicolumn{2}{|l|}{ Costs } & & \multicolumn{2}{|c|}{ 95\% Cl cost-difference } \\
\hline $\begin{array}{l}\text { Intervention costs in } \\
\text { euros, mean }(S D)^{1}\end{array}$ & $57(4)$ & $6(0.9)$ & 43 & 60 \\
\hline $\begin{array}{l}\text { Time costs in euros, } \\
\text { mean }(S D)^{1}\end{array}$ & $163(12)$ & $155(14)$ & -29 & 44 \\
\hline Effects & & & $\mathrm{t}$ & $P$ \\
\hline $\begin{array}{l}\text { Guideline adherence } \\
(0-9) \text {, mean (SD) }\end{array}$ & $7.7(1)$ & $7.4(2)$ & -1.704 & .090 \\
\hline
\end{tabular}

${ }^{1}$ Mean costs based on complete baseline sample ( $\left.\mathrm{N}=269\right)$ 
Table 5 Costs and effects on patient level at six- and twelve-month follow up

\begin{tabular}{|c|c|c|c|c|}
\hline Six-month follow-up & $\begin{array}{l}\text { Intervention group } \\
(\mathrm{N}=138)\end{array}$ & $\begin{array}{l}\text { Control group } \\
(\mathrm{N}=99)\end{array}$ & & \\
\hline Costs & & & \multicolumn{2}{|c|}{$\begin{array}{l}95 \% \mathrm{Cl} \\
\text { cost-difference }\end{array}$} \\
\hline Healthcare costs in euros, mean (SD) ${ }^{1}$ & $690(83)$ & $535(49)$ & -29 & 357 \\
\hline Patient costs in euros, mean (SD) ${ }^{1}$ & $22(0.8)$ & $23(0.9)$ & -3 & 1 \\
\hline Effects & & & $\mathrm{F} / \mathrm{t}$ & $P$ \\
\hline Continued abstinence (\%) & $60(43.5 \%)$ & $38(38.4 \%)$ & 0.617 & .43 \\
\hline Quit intention (1-7), mean (SD) & $6.3(1)$ & $6.2(1)$ & -0.192 & .85 \\
\hline FTND (0-10), mean (SD)² & $3.1(2)$ & $4.1(2)$ & 2.769 & .01 \\
\hline ICECAP utility, mean (SD) & $0.85(0.2)$ & $0.85(0.1)$ & 0.333 & .74 \\
\hline Twelve-month follow-up & $\begin{array}{l}\text { Intervention group } \\
(\mathrm{N}=113)\end{array}$ & $\begin{array}{l}\text { Control group } \\
(\mathrm{N}=88)\end{array}$ & & \\
\hline Costs & & & \multicolumn{2}{|c|}{$\begin{array}{l}95 \% \mathrm{Cl} \\
\text { cost-difference }\end{array}$} \\
\hline Healthcare costs in euros, mean (SD) ${ }^{1}$ & $390(39)$ & $536(87)$ & -339 & 41 \\
\hline Patient costs in euros, mean (SD) $)^{1,3}$ & $9(0.4)$ & $10(0.5)$ & -2 & 0 \\
\hline Effects & & & $\mathrm{F} / \mathrm{t}$ & $P$ \\
\hline Continued abstinence (\%) & $53(46.9 \%)$ & $40(45.5 \%)$ & 0.042 & .84 \\
\hline Quit intention (1-7), mean (SD) & $6.4(1)$ & $6.2(1)$ & -0.989 & .32 \\
\hline FTND (0-10), mean (SD) ${ }^{2}$ & $3.4(2)$ & $3.6(2)$ & 0.463 & .64 \\
\hline ICECAP utility, mean (SD) & $0.87(0.1)$ & $0.84(0.1)$ & -1.522 & .13 \\
\hline
\end{tabular}

${ }^{1}$ Costs during the prior six months

${ }^{2}$ Only patients who smoked at follow-up are included

${ }^{3}$ Only patients who had consultations with their PN between six- and twelve month follow-up are included

On patient level, the CEA demonstrates that in comparison to the control group, $€ 7,126.48$ has to be paid in the intervention group for each additional patient being abstinent at six-month follow-up and that the intervention group is dominated by the control group at twelve-month follow-up (Table 6). The CEAC shows that it is moderately likely that the program is cost-effective after six months and not likely to be cost-effective after twelve months (Appendix 2). Results from secondary conservative analyses (i.e. 'missing=smoking' assumption), showed similar results: costs for each additional patient being abstinent at six-month follow-up are $€ 11,886.17$, and the intervention group is dominated by the control group at twelve-month follow-up. 
Table 6 ICERs and bootstrapped results of cost-effectiveness

\begin{tabular}{lllll}
\hline & ICER/ICUR & \multicolumn{2}{l}{ Likelihood cost-effectiveness at } \\
\cline { 3 - 4 } & & WTP =€10,000 & WTP = €20,000 & WTP = €50,000 \\
& & $94 \%$ & $96 \%$ & $97 \%$ \\
PN level: societal perspective & $€ 1,585.54$ & $88 \%$ & $94 \%$ & $96 \%$ \\
PN level: program use perspective & $€ 3,588.57$ & $55 \%$ & $64 \%$ & $68 \%$ \\
Patient level: six-month follow-up & $€ 7,126.48$ & $25 \%$ & $26 \%$ & $26 \%$ \\
Patient level: twelve-month follow-up & $€-961.78$ & $37 \%$ & $53 \%$ & $67 \%$ \\
Patient level: ICECAP & $€ 18,431.16$ & $41 \%$ & $54 \%$ \\
Patient level: EuroQol & $€ 33,589.46$ & $30 \%$ & & \\
\hline
\end{tabular}

\section{Cost-utility analyses}

The CUA conducted from a capability perspective (i.e. with ICECAP utility scores) demonstrates that in comparison to the control group, costs per QALY gained are $€ 18,431.16$ in the intervention group. The CUA, from a health-related quality of life perspective (i.e. with EuroQol utility scores), demonstrates that these costs per QALY gained are $€ 33,589.46$ (Table 6). The CEAC shows that at the Dutch WTP threshold of $€ 20,000$ (Raad voor de Volksgezondheid en Zorg, 2006) the intervention is $53 \%$ likely to be cost-effective regarding ICECAP utilities and 41\% likely concerning EuroQol utilities (Appendix 3).

\section{Discussion}

\section{Main findings}

Providing intervention group PNs with access to the e-learning program resulted in higher intervention costs, but also in small increases in effect compared to control group PNs. On PN level, CEA demonstrated that the e-learning program was likely to be cost-effective. Intervention costs were higher for intervention group PNs as control group PNs did not have access to the full e-learning program. Overall time costs were similar between both groups; additional analyses revealed that time costs associated with program use were higher among intervention group PNs (€40 vs. €6), as they engaged more with the e-learning program. As time costs associated with counseling did not differ between the groups, and additional analyses found no significant differences in the number and duration of consultations, the difference in PNs' guideline adherence likely results from an improvement in the quality of counseling, rather than its quantity. The CEA revealed that adherence to each additional guideline step was associated with incremental costs of $€ 1,586$. Although information is currently lacking about society's willingness to pay for such effects (i.e. only WTP thresholds per QALY gained are available (Raad voor de 
Volksgezondheid en Zorg, 2006)), our bootstrapped results were consistent across several WTP thresholds. Hence, providing PNs with an e-learning program seems worth the investment to achieve an improvement in smoking cessation counseling. As the quality of PN-led care is known to be comparable to GP-led care (Dierick-van Daele et al., 2009; van Rossem et al., 2017) and has the potential to be cost-saving (Swan et al., 2015), providing PNs with this e-learning program will improve chances that $\mathrm{PN}$-led care will also be cost-effective in Dutch general practice. Hence, it is recommended to pursue widespread program use by PNs across the Netherlands. However, as program implementation entails challenges (Ahmed, Dannhauser, \& Philip, 2018; Joseph, West, Shickle, Keen, \& Clamp, 2011; van de Glind et al., 2015), systematic implementation research is required to ensure the development of an evidence-based implementation plan. Such a plan should be supported by the program's target group and other relevant stakeholders to develop a practically feasible implementation approach (van Gemert-Pijnen et al., 2011; van Limburg, Wentzel, Sanderman, \& van Gemert-Pijnen, 2015).

On patient level, no differences in costs and smoking abstinence were found after six and twelve months, although after six months lower FTND scores were found among intervention group patients - an effect that disappeared after twelve months. Consequently, for patients the e-learning program was moderately cost-effective after six months, yet not cost-effective after twelve months. This finding was confirmed by CUA using capability-related and health-related quality of life as outcome measures. An explanation for finding little effects on patient level, could be that many patients relapsed back to smoking after making a quit attempt (Marlatt \& Donovan, 2009). More specifically, as a result of the e-learning program, intervention group PNs might have been more motivated to engage in counseling with a wider range of smoking patients, including patients that were more nicotine dependent. As nicotine dependence is known to predict quit success (Vangeli, Stapleton, Smit, Borland, \& West, 2011), these patients first needed to lower their dependence before making a quit attempt, which is supported by our results showing improvement in FTND scores among intervention group patients after six months. As this effect on FTND scores disappeared after twelve months, this could suggest a delayed relapse back to smoking among intervention group patients between sixand twelve-month follow-up. Future research should focus on preventing smoking relapse among patients who are counseled by a PN in general practice. PNs could, for instance, explore possibilities to refer patients to complementary smoking relapse interventions (Collins, Witkiewitz, Kirouac, \& Marlatt, 2010) in addition to PN-led counseling. PNs could actively refer patients to addition evidence-based, behavioral support focusing on training coping skills (Chirikos, Herzog, Meade, Webb, \& Brandon, 2004; Elfeddali et al., 2012; Unrod et al., 2016) or could recommend additional pharmacotherapeutic support (Agboola, McNeill, Coleman, \& Bee, 2010; Coleman et al., 2010), known to be effective to prevent smoking relapse. By supporting smokers to additionally use such effective relapse prevention strategies it could be possible to achieve improved abstinence rates that are sustained over a longer time period. 


\section{Strengths and limitations}

Results from this economic evaluation of our novel e-learning program for PNs can be used by health policy makers to inform decisions on smoking cessation investments. In line with recent recommendations, an assessment of quality of life from a broader perspective than health-only was used (i.e. capability-related quality of life), enabling comparison of our results with economic evaluations in other domains (Smit, de Vries, Oberjé, \& Evers, 2015). Nevertheless, no effects concerning quality of life were found, which could be the result of the twelve-month follow-up period not being sufficiently long to detect changes in patients' quality of life, which is often attributed to patients experiencing withdrawal symptoms after quitting smoking (Shiffman et al., 2006). Also recent other studies among smoking patients found little or no effects concerning QALYs, measured after similar follow-up periods (Berndt et al., 2016; Smit, Evers, et al., 2013). It is therefore suggested to either extend the period of data collection of trial-based economic evaluations, or to use modelling techniques to predict long-term costs and effects (Smit, de Vries, et al., 2015).

A limitation of this study was the low number of patients that enrolled in the trial (33\%; 388/1,175) and the high number of patients that dropped out of the trial after enrollment (48\%; 187/388). Unfortunately, this seems to be inherent to trials concerning web-based interventions, which often report similar or even worse results (Bennett \& Glasgow, 2009; Eysenbach, 2005; Kohl et al., 2013). Consequently, however, this might have limited the statistical power of our analyses to detect the small differences in patients' smoking abstinence.

\section{Conclusions}

Providing PNs with a tailored e-learning program to support their adherence to the national smoking cessation guideline can be a cost-effective approach to improve PNs' smoking cessation counseling. Unfortunately, results do not show additional, indirect cost-effectiveness on patient level. National implementation of the e-learning program should be pursued as it can improve the quality of smoking cessation care among a larger population of PNs. Yet, future research should focus on strategies to prevent patients' smoking relapse and to reliably assess long-term costs and effects. 
Appendix 1: Cost-effectiveness planes (left) and corresponding CEACs (right) on PN level

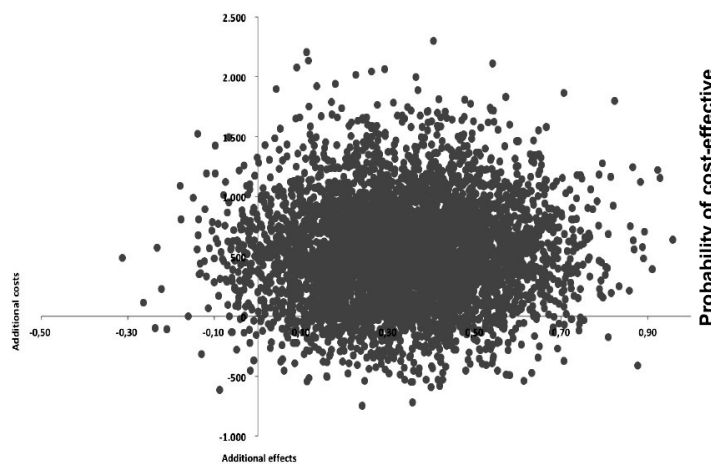

A) CEP on PN level, societal perspective

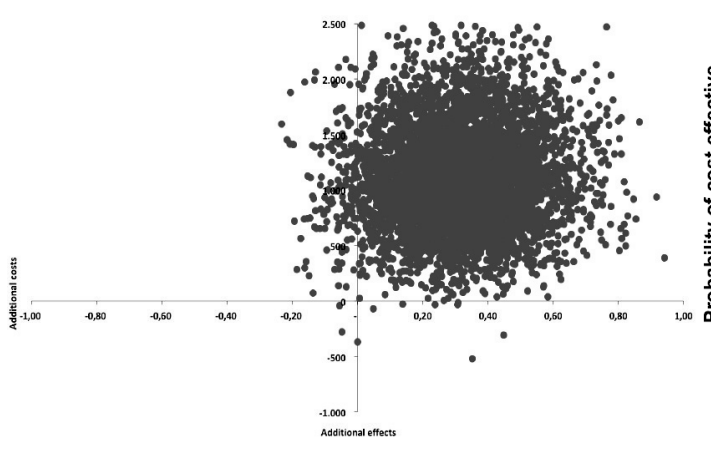

C) CEP on PN level, program use perspective

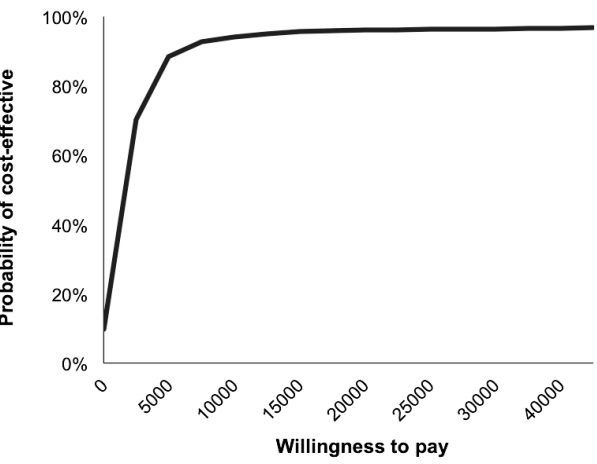

B) CEAC on PN level, societal perspective

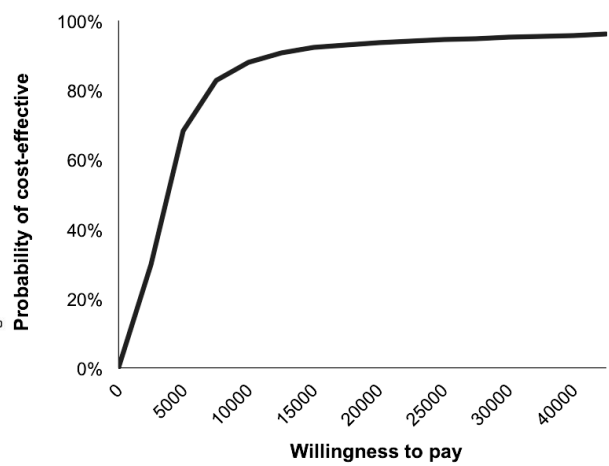

D) CEAC on PN level, program use perspective 
Appendix 2: Cost-effectiveness planes (left) and corresponding CEACs (right) concerning abstinence at smoker level
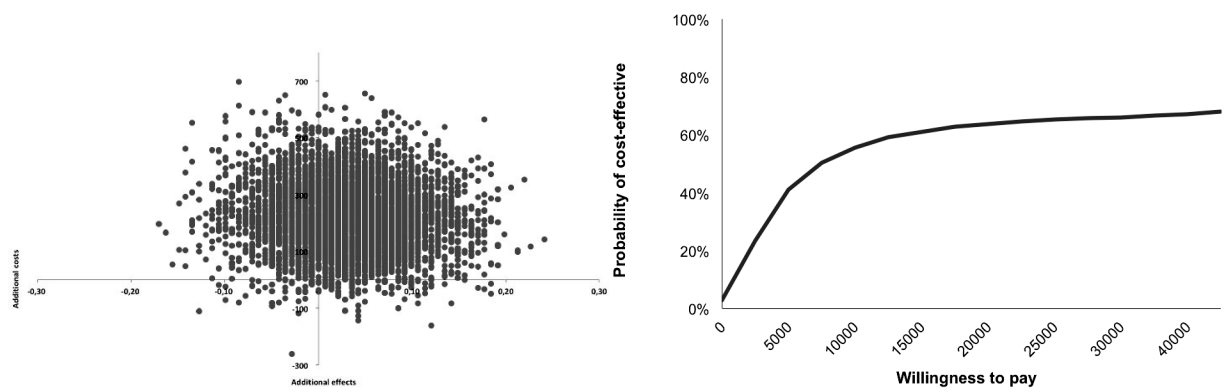

A) CEP on patient level, six-month follow-up

B) CEAC on patient level, six- month follow-up
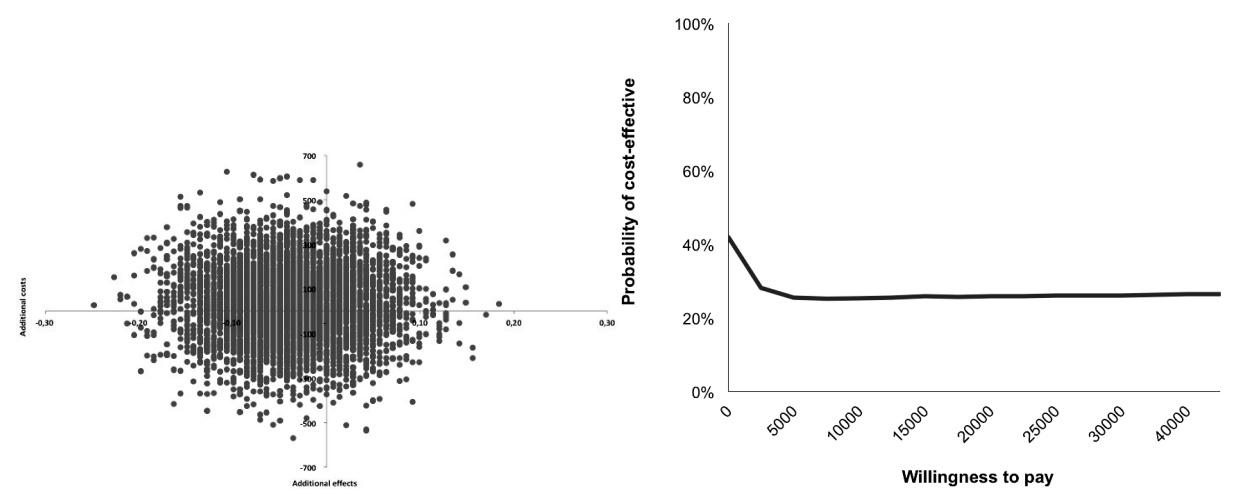

C) CEP on patient level, 12-month follow-up

D) CEAC on patient level, 12-month follow-up 
Appendix 3: Cost-effectiveness planes (left) and corresponding CEACs (right) concerning utilities at smoker level
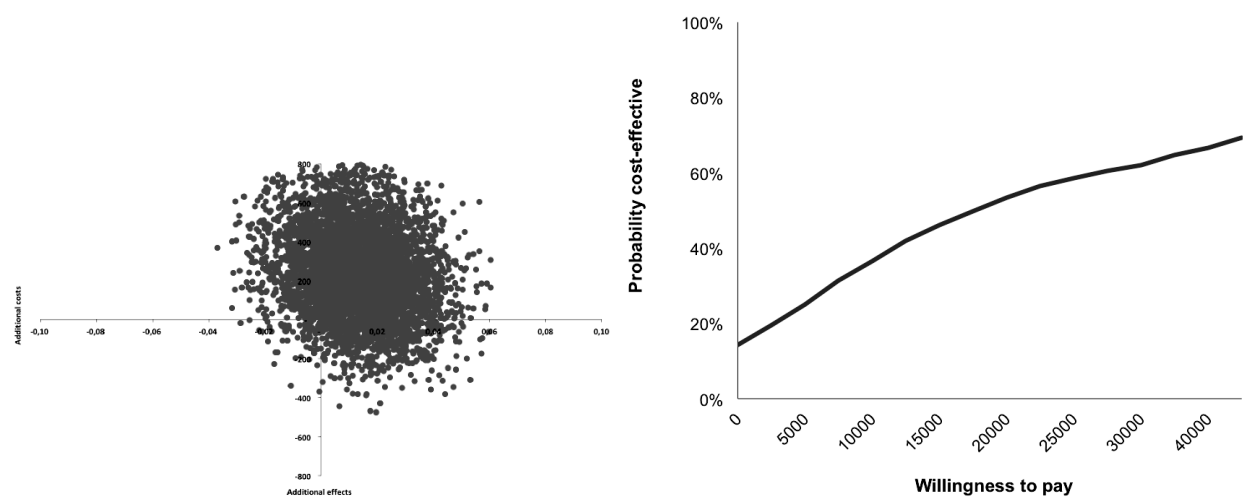

A) CEP on patient level, ICECAP utilities

B) CEAC on patient level, ICECAP utilities
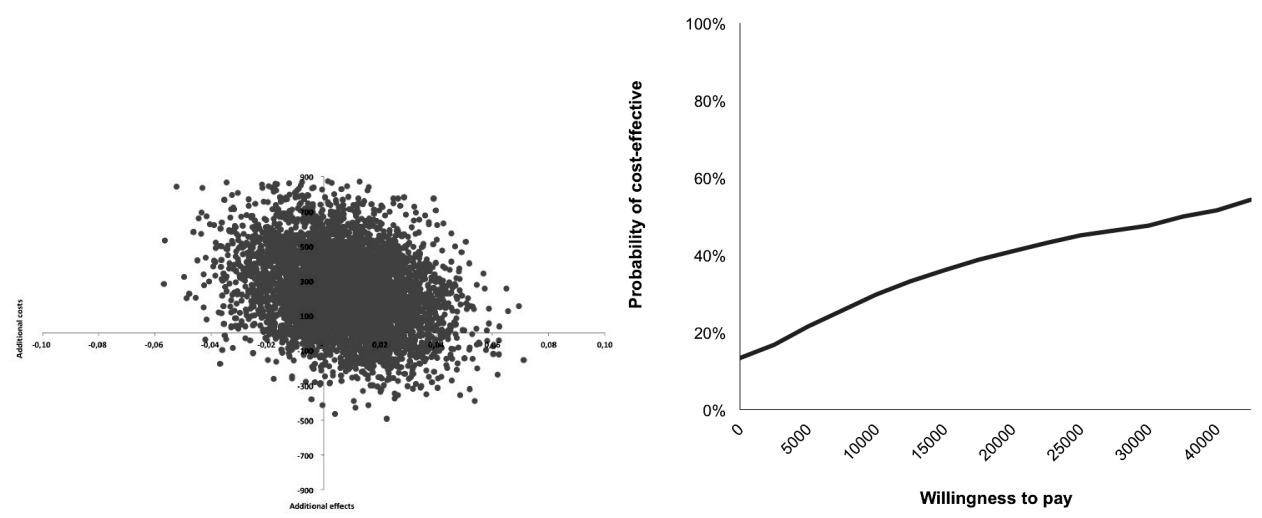

C) CEP on patient level, EuroQol utilities

D) CEAC on patient level, EuroQol utilities 

Chapter

General discussion 
The overall aim of the project described in this dissertation was to provide detailed insight in the (determinants of) current smoking cessation counseling practices of practice nurses (PNs) in Dutch general practice and to develop a web-based computer-tailored elearning program based on these findings. Subsequently, the program's (cost-)effectiveness was evaluated. The chapters in this dissertation are therefore clustered around two topics: 'developing guideline adherence support' and 'evaluating guideline adherence support'. In the first part of this general discussion, for each topic project results are discussed in terms of research and practice implications. The second part of the general discussion addresses overall methodological, theoretical, and practical considerations of the findings. Finally, general conclusions and recommendations are presented.

\section{Developing guideline adherence support}

\subsection{Main findings}

The parts of this dissertation addressing 'developing guideline adherence support' were described in chapters 2-4. The results of each chapter are first summarized and subsequently main results are discussed.

Chapter 2: exploring practice nurses'smoking cessation counseling practices and needs for support

Our results showed that all PNs reported to use an evidence-based guideline, but that they also experienced difficulties in optimally applying such a guideline in practice. A prominent psychological barrier was PNs' low self-efficacy to fully adhere to the guideline, whereas frequently mentioned practical barriers were PNs' perception of a lack of high-quality smoking cessation training opportunities and difficulties in obtaining up-todate information (e.g. on rules and regulations concerning compensation of counseling for smokers). PNs expressed interest in a guideline adherence support program, specifically an easy-to-use program that could directly be consulted and applied in practice, against minimal time investment.

Chapter 3: describing factors associated with practice nurses' guideline adherence PNs' adherence was described as their overall adherence to the national guideline and as their adherence to individual guideline steps (i.e. step-based adherence). Overall adherence ( 5 out of 9 steps on average) was positively associated with higher levels of selfefficacy to use a guideline and spending more time on counseling. PNs' step-based adherence ranged between $34-75 \%$ (e.g. consistent application of the guideline step 'providing a quit advice' was 57\%), which was similarly associated with self-efficacy to use a guideline and spending more time on counseling, and with perceiving more advantages of using a guideline. 
Chapter 4: describing development of the support program

Results from chapter 2 and 3 were used as input for the development of a web-based computer-tailored e-learning program to support Dutch PNs to improve their smoking cessation guideline adherence. Program content and design were based on PNs' adherence support needs and perceived barriers (e.g. content segmentation to limit time investment), their socio-cognitive determinants of adherence (e.g. self-efficacy towards guideline use) and usability evaluations of the program from both PNs and experts (e.g. improving the program's navigation structure).

\subsection{Research implications}

\section{Guideline adherence by practice nurses}

The results of the studies reported on in chapters 2 and 3, revealed that not all guideline steps were equally well adhered to. Especially providing a quit advice and more active counseling steps (i.e. increasing smoker motivation, assessing and removing smokers' barriers, discussing smoking cessation aids, and setting up a quit plan) were sub-optimally applied in practice, which is in line with quantitative studies among hospital nurses (Berndt et al., 2013; Segaar, Willemsen, et al., 2007) and a qualitative study among PNs (van Rossem et al., 2015).

PNs' low adherence to providing a quit advice can potentially be explained by the fact that general practitioners (GPs) already provided a quit advice to patients before referring them to PN-led structured counseling. It is then likely that PNs subsequently do not repeat such an advice when starting a counseling trajectory. Although PNs in our project indeed expressed it as helpful when their GP actively provided a quit advice, they also reported that this did not happen enough in practice. We did not assess whether GPS provided a quit advice to patients before referring them to a PN, to limit the burden on GPs and because involving GPs in research is challenging (Salmon et al., 2007). Studies collecting GP data via questionnaires were confronted with low participation rates of, for instance, 15.6\% (120/771) (Hoving, Mudde, \& de Vries, 2006) and 37.6\% (264/703) (Lugtenberg, Burgers, Besters, Han, \& Westert, 2011). An option for future research may be to collect data on GP's guideline adherence via the practice's digital information system, in which primary healthcare professionals register patient information (de Boer \& Bosman, 2018), or via their PN (e.g. by asking PNs to report on their GP's counseling activities). This may prevent overlooking of opportunities for smoking cessation advice by both GP and PN. This is important as a both a GP's advice (Stead et al., 2013) and advice from a nurse are independently effective to support patients to quit smoking (Rice et al., 2017). Yet, whether repetition of a quit advice by both GP and PN is even more effective is not known yet. Therefore, it is recommended to investigate whether smoking patients receive 1) a single quit advice from either GP or PN, 2) a double quit advice from both their GP and PN, or 3) no quit advice at all, and determine the effect of each scenario on a patient's quit motivation and quit success. Evidence on the effectiveness of a double 
advice could yield important information about the potential effectiveness of co-counseling by a GP and a PN. This can be used to provide primary healthcare professionals recommendations about how to jointly apply smoking cessation counseling in general practice, which could hence improve adherence to the evidence-based guideline on practice level.

An alternative explanation for why PNs refrain from providing a quit advice is that they might want to prevent patient dissatisfaction by damaging their relationship with the patient (Baummer-Carr \& Nicolau, 2017). Concerns about the patient-provider relationship indeed predicted the provision of a quit advice in a study among British GPS (McEwen, West, \& Preston, 2006) and a Dutch study found that smokers more often made negative than positive statements about quitting smoking when their GP or PN addressed this topic (Verbiest, Chavannes, et al., 2014). However, a qualitative study among Dutch GPs and PNs found that they were actually willing to jeopardize their patient-provider relationship by talking about smoking (van Rossem et al., 2015). An explanation can be sought in the strength of the patient-provider relationship at the onset of a smoking cessation counseling trajectory. Evidence suggests that patients find it easier to talk to nurses compared to a GP or medical specialist (Henselmans, Heijmans, Rademakers, \& van Dulmen, 2015) and describes positive results of patient-nurse communication on patient outcomes (de Leeuw et al., 2014; Gunther \& Alligood, 2002; Macdonald, 2016). This suggests that a strong patient-provider relationship may improve patient-PN communication. However, currently evidence on the role of the patient-PN relationship is lacking. Further research should explore the strength of the patient-PN relationship concerning smoking cessation counseling to understand how this relationship influences PNs' guideline adherence. This could, for instance, be achieved by evaluating video-records of PNled smoking cessation consultations, a method previously proven to be feasible among primary care midwives (Spelten et al., 2015).

PNs' difficulties to adhere to guideline steps that follow the provision of a quit advice (e.g. increasing smoker motivation, assessing and removing smokers' barriers) could partially result from PNs not always providing a quit advice in the first place, as the provision of a quit advice could facilitate starting a conversation about smoking cessation with a patient. Nevertheless, PNs' reported that their adherence is also influenced by a patient's difficulties to become motivated to quit smoking. Although the national smoking cessation guideline encourages healthcare professionals to adapt their counseling to the motivational stage of a patient, based on the Stages of Change (Prochaska \& Velicer, 1997), the guideline does not explain how they should deal with other differences between patients which could influence their quit motivation and PNs' counseling (Trimbos-instituut \& NHG, 2016). For example, it was recently recommended to incorporate an identitycomponent in smoking cessation interventions to differentiate between patients with a smoker and a non-smoker self-identity (Meijer et al., 2018). Meijer et al. (2018) found that patients who identify themselves more as a non-smoker than a smoker are more likely to receive a quit advice and make a quit attempt, compared to patients with a 
smoker self-identity. These results illustrate that strategies to promote a patient's selfidentification as a non-smoker (e.g. PNs discussing with patients why smoking or nonsmoking fits with them) may improve the effectiveness of PNs' smoking cessation counseling. Further research is needed to establish the effects of incorporating an identitycomponent in smoking cessation counseling on improving PNs' provision of a quit advice (and application of subsequent guideline steps) and patients' quit attempts (and quit success).

The influence of patient motivation on practice nurses' counseling

The positive association we found between a PN's level of self-efficacy and their guideline adherence corroborates results from other studies among PNs (Smit, de Vries, et al., 2013; Whitehead et al., 2014). Moreover, PNs' low level of self-efficacy was explained by their struggle to realize a balance between motivating smokers and convincing smokers that becoming motivated is their own responsibility (de Ruijter, Smit, de Vries, Goossens, et al., 2017). Some PNs shifted motivational responsibilities to their smoking patients, resulting in stopping counseling when a patient's motivation was not yet sufficient to quit (van Rossem et al., 2015). These qualitative findings suggest that patients' low motivational status may negatively influence PNs' level of self-efficacy and subsequent guideline adherence. As smokers especially struggle with becoming sufficiently motivated to quit (de Hoog et al., 2016; Papadakis, Tulloch, Gharib, \& Pipe, 2016), PNs' lack of counseling when smokers' motivation to quit is low, is a missed opportunity to support patients during this process. Hence, to further improve PNs' smoking cessation guideline adherence it is important to quantitatively investigate the influence of patients' level of quit motivation on PNs' counseling. Such research should include multiple moments of assessment to determine causality between constructs measured at PN and patient level (Flay, 1986). Consequently, a longitudinal study could be conducted to simultaneously investigate changes in PNs' guideline adherence and patients' motivation over time. More specifically, patient motivation could be assessed before and after the intake consultation with their PN, and during subsequent consultations with their PN (both up to and after a quit date). These data should then be analyzed in combination with data on PN's guideline adherence to explore whether and how the influence of patient motivation on PNs' guideline adherence changes during a complete counseling trajectory. This would yield valuable, additional insights into PNs' guideline adherence and opportunities to improve smoking cessation counseling for patients. Patients could, for instance, be offered additional motivation-enhancing support to increase the likelihood that their quit motivation is sufficiently high for PNs to engage in structured cessation counseling with their patients. When such additional patient support is offered via the Internet this enables patients to efficiently use it in addition to PN-led counseling (i.e. blended care), an approach that was already successful to improve patients' self-management (Smeets \& ZijlstraVlasveld, 2016; Talboom-Kamp, Verdijk, Kasteleyn, Numans, \& Chavannes, 2018). Yet, to 
determine the added benefit of introducing blended care in general practice, more research on the optimal combination of PN-led counseling and web-based patient support is recommended (Smit et al., 2016; Talboom-Kamp et al., 2018).

\subsection{Practical implications}

Implementation of technological developments in general practice

The emergence of novel possibilities for intervention development, such as our computer-tailored e-learning program for PNs, go hand in hand with technological advancements in all aspects of life (Patrick et al., 2016). Developments such as wearables, social media and big data provide endless opportunities to measure, analyze and disseminate all kinds of data with great potential to develop interventions aimed at promoting a healthier society (Michie et al., 2017). Interventions using new digital technologies to promote health (e.g. telemedicine, mHealth) and to provide electronic ways of learning (e.g. e-learning) are increasingly becoming integrated in daily routines for science and practice (from here on, these interventions are collectively referred to as eHealth interventions). Within scientific research, a large number of eHealth interventions is continuously being developed, tested and reported on. To illustrate, two systematic reviews of literature reviews were conducted about the effectiveness of eHealth interventions that were published between 2005-2012, identifying a total of 121 literature reviews (Ekeland, Bowes, \& Flottorp, 2010; Elbert et al., 2014). Even an eHealth version of the Consolidated Standards of Reporting Trials (CONSORT-EHEALTH) statement has been developed to facilitate reporting of trials including eHealth technologies (Eysenbach, 2011), demonstrating the growing importance of eHealth interventions in scientific research. This is also observed in practice, as numerous eHealth interventions have demonstrated their effectiveness in improving health-related or care-related outcomes, for instance in the treatment of obesity (Hutchesson et al., 2015), asthma (Zhao, Zhai, Zhu, \& Sun, 2015), or heart failure (Kotb, Cameron, Hsieh, \& Wells, 2015). As a result of the widespread availability of eHealth interventions and their integration in everyday life, it means that most healthcare professionals likely already have experience in using some form of eHealth. However, this does not automatically mean that they will have a favorable attitude towards eHealth and will decide to use an eHealth intervention in practice (Lau et al., 2016; Voogt, Opmeer, Kastelein, Jaspers, \& Peute, 2018). Therefore, to increase the uptake of eHealth interventions and to optimize integration of these interventions in practice, it is proposed to use a multidisciplinary approach for developing eHealth interventions, including stakeholder involvement (van Gemert-Pijnen et al., 2011).

To ensure stakeholder involvement during the development of our e-learning program, PNs were consistently part of the studies that were required for program development, resulting in a program with optimal usability characteristics (e.g. low complexity) and as compatible with PNs' daily practice as possible. However, optimizing the program's compatibility was rather challenging, as individual general practices differ in the type of 
digital information system used to manage patients' health records (de Boer \& Bosman, 2018). Ideally, eHealth interventions are integrated in existing information systems to enable easy access and prompting healthcare professionals to use the intervention (Lau et al., 2016). Due to the multitude of information systems used in Dutch general practice, it is however difficult to develop eHealth interventions with universal compatibility with these systems. This results in the development of multiple stand-alone eHealth interventions that are each compatible with a limited set of information systems or that circumnavigate existing systems altogether (such as web-based programs) (de Boer \& Bosman, 2018). This makes it more difficult for healthcare professionals to use these interventions, because interventions are rather stand-alone instead of compatible with each other, as well as with current practice. Novel developments, such as integrated eHealth platforms that harbor several eHealth interventions in one system (Huygens, 2018; Swinkels et al., 2018), can potentially increase the uptake of eHealth interventions in general practice. Such platforms facilitate the use of multiple eHealth interventions simultaneously, which are all compatible with current practice within one platform. Creation of an integrated platform and the development of eHealth interventions accordingly, are therefore recommended for Dutch general practice.

\section{Evaluating guideline adherence support}

\subsection{Main findings}

The parts of this dissertation addressing 'evaluating guideline adherence support' were described in chapters 5 and 6 . The results of each chapter are first summarized and subsequently main results are discussed.

Chapter 5: effects of the e-learning program on practice nurses' guideline adherence A randomized controlled trial (RCT) was conducted among Dutch PNs and smokers counseled by these PNs to assess the e-learning program's effectiveness to improve PNs' guideline adherence. The effect analyses results showed that PNs in the intervention group who were already more experienced in providing counseling, reported better overall adherence to the smoking cessation guideline compared to control group PNs with less experience. Concerning step-based adherence, it was found that for PNs with less favorable baseline scores on behavioral predictors (e.g. low levels of self-efficacy and perceived advantages) and high baseline levels of counseling experience, the program significantly increased adherence.

Chapter 6: cost-effectiveness and cost-utility of the e-learning program

The economic evaluation of the e-learning program was conducted on PN and patient level. On PN level, the cost-effectiveness results illustrated that both from a societal and 
from a program use perspective, the e-learning program was very likely to be cost-effective in improving PNs' guideline adherence. On patient level, a significant reduction was found in intervention group patients' nicotine dependence after six months, but this effect disappeared after twelve months. Cost-effectiveness analyses on patient level showed that the e-learning program was moderately likely to be cost-effective after six months, and not cost-effective after twelve months to improve patients' smoking abstinence. Cost-utility analysis on patient level also demonstrated moderate likelihood of the program's cost-effectiveness to improve patients' quality of life. In conclusion, the elearning program was cost-effective to improve PNs' guideline adherence, but not costeffective to indirectly improve patients' smoking abstinence.

\subsection{Research implications}

\section{Working experience of practice nurses}

One of the main findings of the effect evaluation concerns the role of PNs' working experience; more experienced PNs seemed to benefit more from the program, compared to less experienced PNs. This is in line with findings from chapter 3, demonstrating an association between the amount of time spent on counseling (as a result of counseling more patients) and better guideline adherence. The underlying mechanism may be that more frequent engagement in smoking cessation counseling is related to a higher level of smoking cessation guideline adherence and related to PNs benefiting more from an adherence support program. Nevertheless, other recent studies do not support this mechanism. A study among physicians, for example, found no relationship between experience and guideline adherence (Nicastro et al., 2015), and another study even found that Dutch child healthcare professionals with more years of experience were less likely to adhere to national guidelines compared to less experienced colleagues (Konijnendijk, BoereBoonekamp, Fleuren, Haasnoot, \& Need, 2016). Additional PN data is required to better understand the influence of PNs' level of experience on their guideline adherence. For example, the degree of integration of guideline use in daily work routine is known to influence guideline implementation in primary care (Lau et al., 2016). Experienced PNs in our trial may have had a higher degree of routine guideline use compared to less experienced PNs, thereby explaining better guideline adherence. This mechanism is supported by findings that describe difficulties to get experienced healthcare professionals to adhere to a guideline which is not part of their daily routine (Konijnendijk et al., 2016). Yet, in order to understand this mechanism concerning PNs' smoking cessation counseling, additional data is required about how long a PN already uses a guideline and the degree to which guideline use has become part of their counseling routine.

Alternatively, the range in level of PNs' professional education might be relevant to explain the moderating role of PNs' work experience (Freund et al., 2015). Based on data from our trial, we found that more experienced PNs ( $\geq$ mean +1 SD) were on average 10 years older than less experienced ( $\leq$ mean -1 SD) and 3 years older than PNs with an 
average level of experience. Moreover, research has illustrated that many young PNs have completed different professional education compared to older generation of PNs; younger PNs more often have completed a nursing bachelor, whereas older PNs more often have a background in vocational education as a nurse or physician assistant before their PN training (van Hassel et al., 2016). Because of this difference in professional education, older PNs in our trial likely have more field experience than young PNs, as they have already been in working in general practice as a nurse or physician assistant. Since research among Dutch PNs and physician assistants found that they are involved in many similar patient care activities, including lifestyle counseling (Noordman, Verheij, \& Verhaak, 2008), this could explain why older, more experienced PNs in our trial reported better adherence scores. Further research should be conducted to corroborate the relationship between PNs' professional education and their guideline adherence, and to explore how our e-learning program can be improved accordingly to also be beneficial for PNs with less practice experience.

\section{Improving the quality of smoking cessation care}

RCT results indicated that the e-learning program improved self-reported smoking cessation guideline adherence among experienced PNs in the intervention group, but that this did not lead to significant improvements in smoking abstinence among their counseled patients. Furthermore, additional analyses among counseled patients revealed that patients' perceived quality of care, measured by the Health Care Climate Questionnaire (Williams, Grow, Freedman, Ryan, \& Deci, 1996), did not differ when comparing counseling from 1) intervention or control group PNs, and 2) more or less experienced PNs. It hence seems that - next to the e-learning program - additional strategies are required to increase patients' smoking abstinence rates, e.g. through improving the quality of PNs' counseling, as this is known to benefit patient outcomes (Melnyk, Gallagher-Ford, Long, \& Fineout-Overholt, 2014).

A frequently applied strategy to improve the quality of counseling is to apply principles of motivational interviewing (Miller WR \& Rollnick SR, 2002). As this method is proven to be effective in helping people to quit smoking (Lindson-Hawley, Thompson, \& Begh, 2015; Williams et al., 2006; Williams, Niemiec, Patrick, Ryan, \& Deci, 2009), Dutch PNs are instructed to provide smoking cessation counseling based on motivational interviewing, as part of their professional education (Heiligers et al., 2012) and as recommended by the national evidence-based guideline (Trimbos-instituut \& NHG, 2016). Nevertheless, research has illustrated that PNs find it difficult to apply motivational interviewing techniques (Noordman, Koopmans, Korevaar, van der Weijden, \& van Dulmen, 2013; Noordman et al., 2012), a result also found among nurses in Sweden (Efraimsson et al., 2015). Additional follow-up training programs or workshops are therefore recommended. As motivational interviewing training programs for nurses proved to be effective in improving their skills in a range of counseling situations, such as cardiovascular care (Fontaine et al., 2016) or generic communication skills (Noordman, van der Weijden, \& 
van Dulmen, 2014), it is worthwhile to also investigate the effect of such programs for PNs in general practice. Although our e-learning program included a module with content on motivational interviewing basics, this particular content was rather descriptive (i.e. non-tailored) as it was not the program's main goal to improve and/or support these skills. Several tailored motivational interviewing programs proved to be effective in other care areas, such as promoting patients' level of physical activity (Friederichs, Oenema, Bolman, \& Lechner, 2015) and decreasing patients' level of chemotherapy-related distress (Coolbrandt et al., 2018), but currently no such training program for smoking cessation counseling exists. Hence, further research into the development of a tailored motivational interviewing module could be a valuable addition to the e-learning program in order to improve the quality of PNs' care for smoking patients.

\section{Abstinence among counseled smokers}

The effects of the e-learning program on the level of smoking patients showed that, although differences were non-significant, more patients were abstinent when they were counseled by intervention group compared to control group PNs after six $(43.5 \%$ vs. $38.4 \%$ ) and twelve months (46.9\% vs. $45.5 \%$ ). These small, non-significant differences should be interpreted in light of several limitations. First, patients in both the intervention and control group all received smoking cessation counseling from their PN. As the e-learning program was developed to aim for behavior change at the PN level, the only difference was that intervention group patients received counseling from a PN who had access to the e-learning program for a period of six months. Hence, the trial primarily focused on detecting a difference in PNs' guideline adherence. Due to lower than expected smoker participation in the trial, our analyses were underpowered to also detect the small differences in smoking abstinence between counseled patients. Second, usage of the e-learning program by intervention group PNs was rather limited, suggesting that PNs did not regularly engage with the program. As poor program uptake and implementation are known to negatively influence a program's impact (Glasgow et al., 1999), this might explain the limited and non-significant indirect effects of the e-learning program on patients' abstinence.

Although results on patients' abstinence were small and not significant, the economic evaluation at six-month follow-up still showed that the e-learning program was moderately likely to be cost-effective on patient level when compared to the control group. Nevertheless, the intervention group was dominated by the control group after twelve months. An explanation for these contradictory findings could be that many smokers, counseled by intervention group PNs, relapsed between the six-month and twelve-month follow-up measurements. A reason for this could be that, as a result of the e-learning program, PNs in the intervention group were more motivated to engage in smoking cessation counseling, maybe also addressing smokers with a more moderate or lower intention to quit. As our data indicate that intervention group PNs significantly more often provided their patients with a quit advice and assessed their patients' motivation to quit, 
this could indicate that intervention group PNs were indeed more motivated to engage in counseling of less motivated smokers. An analysis of PNs' change scores on socio-cognitive factors, which are assumed to predict behavioral motivation (de Vries, 2017), also seems to corroborate this: small, favorable improvements on PNs' level of perceived (dis)advantages, self-efficacy, social modeling, social norms and social support may have contributed to enhanced motivation to apply the national smoking cessation guideline. Moreover, although a baseline difference concerning patients' quit intention was not found, intervention group patients did significantly more often reduce their level of nicotine dependence during the trial, which is a known prerequisite for making a successful quit attempt (Vangeli et al., 2011). Although our trial data indicate that intervention group PNs better adhered to several counseling steps, and that their counseled patients became less nicotine dependent, additional research is required to explore the hypothesis that exposure to the e-learning program resulted in PNs counseling a more varied group of smokers in terms of their quit intention.

\subsection{Practical implications}

Usage of the e-learning program by practice nurses

Despite efforts to fulfill PNs' needs and preferences (de Ruijter, Smit, de Vries, Goossens, et al., 2017) and to tailor content to individually relevant determinants (de Ruijter, Smit, de Vries, \& Hoving, 2017), engagement with the e-learning program was relatively low (Table 1). Of all intervention group PNs, only $38.8 \%$ engaged with both the e-learning modules and the online forum. This could partially be explained by our measurement of usage of the e-learning modules, as partial completion of a module was recorded as nonuse in the program's log data. Yet, partial completion could also mean that PNs intentionally used only part of a module while browsing for specific information. Although program usage was measured conservatively, our results are in line with what might be expected from an eHealth trial, as low rates of adoption and use are frequently reported problems concerning eHealth interventions (Bennett \& Glasgow, 2009; Geraghty, Torres, Leykin, Perez-Stable, \& Munoz, 2013; van Gemert-Pijnen et al., 2011). Program usage was evaluated during qualitative exit interviews with seventeen PNs who participated in the RCT. Factors such as 1) time constraints, 2) having other priorities; and 3) a lack of patients enrolling in smoking cessation counseling were reported to be main reasons for not making (more) use of the e-learning program. Especially time constraints and setting priorities are frequently reported barriers towards program implementation in the healthcare sector, also within other domains than smoking cessation (Ariens et al., 2017; van de Glind et al., 2015). It is therefore important to further elaborate on PNs' low program usage to further understand these barriers and explore potential solutions to alleviate them. 
Table 1 Usage of e-learning program modules by intervention group practice nurses $(N=147)$

\begin{tabular}{llll}
\hline E-learning program content & Collection of log data & Usage, mean (SD) & Number of PN-users (\%) \\
\hline E-learning modules: & & & \\
- Tailored advice & Number of topics completed & $1.8(2.7)$ & $78(53.1 \%)$ \\
- Counseling information & Number of topics completed & $1.4(4.7)$ & $39(26.5 \%)$ \\
Online forum & Number of messages read & $4.7(9.1)$ & $60(40.8 \%)$ \\
& Number of messages posted & $0.1(0.4)$ & $12(8.2 \%)$ \\
& Number of replies posted & $0.3(0.8)$ & $19(12.9 \%)$ \\
\hline
\end{tabular}

A low rate of usage of the e-learning program should be evaluated in the light of two types of attrition: non-usage attrition (i.e. not using the program but remaining in the trial) and drop-out attrition (i.e. complete withdrawal from the trial) (Eysenbach, 2005). Our results showed that among PN drop-outs the level of usage of the e-learning modules (mean number of modules completed: 0.3 vs. 1.7) and the online forum (e.g. number of reads: 0.3 vs. 5.6) was significantly lower than among retained PNs. Moreover, when excluding PN drop-outs from analyses of log data, $\mathrm{PNs}^{\prime}$ usage of the e-learning program slightly increased (e.g. now, 58.7\% completed at least one tailored advice module and $46.3 \%$ read at least one message on the forum). These numbers indicate that drop-out attrition only plays a minor role in explaining low program usage by PNs in the trial, which is supported by the generally low attrition rate of PNs (i.e. $17.7 \%$ in the intervention and $15.6 \%$ in the control group after six months). Although these attrition rates are rather low compared to reports from eHealth trials among patients, reporting attrition rates as high as 60\%-80\% (Bennett \& Glasgow, 2009), recent trials among nurses reported attrition rates of below $20 \%$ as well (Hersch et al., 2016; Saunders, Vehviläinen-Julkunen, \& Stevens, 2016). Hence, non-usage attrition may be the most important attrition factor to understand $\mathrm{PNs}^{\prime}$ low usage of the e-learning program. To prevent non-usage attrition, the content of the e-learning program was presented in a flexible format, meaning that PNs could (repeatedly) select a topic to read small pieces of content of their choice. This was expected to enable PNs to efficiently read program content despite time-constraints at work, which is a frequently mentioned reason for nurses to prefer an e-learning program over a face-to-face training session (de Ruijter, Smit, de Vries, Goossens, et al., 2017; Karaman, 2011). Still, PNs' program usage was limited. During exit interviews, PNs suggested improvements of the e-learning program to stimulate program use: reducing the length of textual program content and increasing in the number of reminders to (re)visit the program.

\section{Program length}

Despite our efforts to develop an e-learning program that was easy-to-use in practice, PNs still perceived that the e-learning program contained too much textual information. Although the tailored advice only included information relevant for the individual PN, more extensive textual feedback could be offered as a result of a PN having less optimal scores on multiple determinants and their behavioral performance (de Ruijter et al., 
2016). As reducing the extent of tailoring could also reduce the program's effect on PNs' adherence, alternative strategies such as increasing the attractiveness and relevance of the format the information is provided in could be explored (Houts, Doak, Doak, \& Loscalzo, 2006). Text-based advice could, for instance, be provided in a format with a higher degree of interactivity (e.g. tailored video feedback), as interactivity is positively related to learning outcomes (Cook et al., 2010). Tailored video feedback proved to be an effective tool to improve both PNs' motivational interviewing skills and generic communication skills (Noordman et al., 2014) and was found to be a more desirable format compared to text-based feedback, based on positive user evaluations (e.g. content acceptability and usefulness) (Soetens, Vandelanotte, de Vries, \& Mummery, 2014; Walthouwer, Oenema, Lechner, \& de Vries, 2015). Video feedback for PNs could focus on their application of the evidence-based guideline during consultations with smoking patients and provide tailored suggestions in video-format on how to practically improve their guideline adherence. Yet, as video feedback might not be preferred by all PNs, one could additionally apply mode tailoring - meaning that the feedback format is individually tailored - to ensure PNs receive information in a format that they prefer (Smit, Linn, \& van Weert, 2015). Mode-tailoring is known to increase users' satisfaction with online interventions (Nguyen, Smets, Bol, Loos, \& Van Weert, 2018), but its effect has so far not been explored in healthcare professionals.

\section{Reminders}

PNs' second suggestion to improve program use was to send them more reminders to (re-)visit the program. Although sending reminders can be effective in facilitating behavior change (Fry \& Neff, 2009), some receivers can also perceive it as too much interference or it can become routine to the receiver, thereby undermining their effectiveness (Vervloet et al., 2012). Therefore, we limited the number of reminders during the trial; PNs received email reminders to stimulate questionnaire completion (i.e. one invitation message and maximum two reminders) and to prompt them to visit the e-learning program (i.e. control group PNs were reminded to use the counseling checklist and intervention group PNs were additionally prompted to use the e-learning modules and online forum), and a single telephone contact was used to offer support and to stimulate program use. Additional stimulation of program use by sending more reminders should perhaps take PNs' individual preferences into account (e.g. optimal number of reminders, communication via email, text message or telephone), to make effective engagement more likely (Yardley et al., 2016). Reminders could be further optimized by applying concepts like context-triggered support (Naughton et al., 2016) or real time monitoring (Vervloet et al., 2011). Both approaches use continuously collected user-data (e.g. location data, behavioral data) to determine optimal moments in time to send reminders. Context-triggered support to prevent smoking relapse proved to be reliable and was positively evaluated by users (Naughton et al., 2016), and real-time monitoring was effective to improve patients' diabetes self-management (Vervloet et al., 2014). For PNs, a similar approach 
could be applied by sending a reminder (e.g. via email or text message, based on their preference) at a moment in time at which it is convenient for them to actually visit the elearning program, for instance based on a PN's location (e.g. when entering the general practice, based on their phone's GPS function) or behavior (e.g. when having a smoking cessation consultation scheduled, based on their electronic schedule). Although this approach could fulfill PNs' need for more reminders, its practical feasibility in Dutch general practice should be explored first.

Patient participation and drop-out during the trial

Many eHealth trials suffer from low rates of participation and high drop-out (Bennett \& Glasgow, 2009; Eysenbach, 2005; Kohl et al., 2013), which was especially observed among smoking patients in our trial. To prevent low participation and high drop-out, several recommended and evidence-based strategies were used during the RCT: 1) offering an incentive upon completion of all questionnaires; 2) sending email reminders to prompt questionnaire completion; 3) assuring anonymous data collection and storage; and 4) limiting the time required to fill out each questionnaire (Bennett \& Glasgow, 2009; van Gelder, Bretveld, \& Roeleveld, 2010).

Despite these strategies, only 33.3\% $(391 / 1,175)$ of counseled smokers participated in the RCT, which might be related to the practical procedure of patient recruitment via participating PNs. To limit the burden for both PNs (e.g. recruitment was minimally timeconsuming) and patients (e.g. little personal data collection), PNs only had to register a patient's date of birth and email address via the e-learning program. This enabled us to create an anonymous patient account and to contact patients via automatically generated email messages. PNs counseled many smoking patients, but several aspects of PNled recruitment might have negatively influenced patient participation. First, the research team could only establish email contact with patients, ruling out more personal communication that could potentially increase patient participation (Bennett \& Glasgow, 2009; Page \& Persch, 2013). Second, PNs' recruitment (i.e. providing trial information and inviting patients to participate) was not monitored, meaning that PNs' 'success rate' is unknown. As the potential reach of smoking patients in general practice is very high (Kleinjan et al., 2016), data on the actual reach would provide insight in the success of our recruitment strategy. To optimize patient participation, it could be important to invest in establishing personal contact (i.e. between patients and researchers) and to monitor PNs' recruitment procedure.

After enrollment, 201 smoking patients were still in the trial at twelve-month followup, resulting in a level of drop-out attrition (48.6\%) that is not uncommon in patient eHealth trials (Bennett \& Glasgow, 2009). Nevertheless, this unfortunately limited our data collection on patient level to only $17.1 \%$ of all patients that were counseled by a PN, which reduced the statistical power to conduct multilevel effect analyses (de Ruijter et al., 2018). An explanation could be that patients' high degree of anonymity (i.e. only PNs had personal patient contact) made it easier for them to drop out of the trial (Duffy, 2002; 
Eysenbach, 2005). Furthermore, our web-based questionnaires were designed to optimize patients' responses by spreading sets of questions across different pages (Toepoel, Das, \& Van Soest, 2009). Although this benefits the quality of collected data and results in higher respondent satisfaction, this could also lower completion rates as patients cannot estimate questionnaire length (van Gelder et al., 2010). Providing patients with information about questionnaire length, such as adding a progress bar (Matzat, Snijders, \& van der Horst, 2009), could optimize patients' questionnaire completion. Yet, since adding a progress bar to a lengthy web-based questionnaire also increases the risk for dropout attrition (van Gelder et al., 2010), it is recommended to carefully pilot-test such strategies with patients before implementing them in a trial.

\section{Methodological and theoretical considerations}

\section{Measuring adherence to the evidence-based guideline}

To determine effectiveness of the e-learning program we intended to use a triangulated measure of guideline adherence in order to limit the risk of bias of relying on PNs' selfreport only (Adams, Soumerai, Lomas, \& Ross-Degnan, 1999). This triangulated measure was meant to include self-reported scores of PNs' adherence both from a PN perspective (i.e. collected via counseling checklists during the trial) and a patient perspective (i.e. collected via online patient questionnaires) (Reif et al., 2011). To assess reliability of the triangulated measure, we intended to compare it with a more objective assessment by creating an adherence score based on audio-recordings of smoking cessation consultations. This could provide additional insight into the congruence between the two different measures and explain their potential convergent or divergent nature (Moran-Ellis et al., 2006).

Unfortunately, collecting adequate data for creating a triangulated measure proved to be very difficult in our trial, making it impossible to create a triangulated measure of PNs' guideline adherence. Firstly, only 54 PNs successfully recruited smoking patients for trial participation, meaning that no data about PNs' guideline adherence could be collected from a patient perspective for $79.9 \%$ of PNs. Secondly, the limited available patient data appeared to suffer from possible ceiling effects as patient-reported data was consistently found to result in significantly higher adherence scores than PN-reported data. More insight into this disagreement between measures could have been obtained by comparing both measures to a more objective measure (Reif et al., 2011), such as the calculation of an adherence score by multiple independent coders using transcripts of audio-recordings of smoking cessation consultations. However, due to resistance from PNs and patients, we only managed to record consultations for only $11.5 \%$ of participating PNs. PNs perceived that audio-recordings lead to privacy concerns and would not pro- 
vide a fair and reliable insight into their regular counseling approach, which are commonly reported challenges for making recordings in healthcare settings (Moloczij et al., 2017; van Bruinessen et al., 2017). Our audio-recordings thus resulted in too little data to use for triangulation purposes.

A method for collecting data on PNs' guideline adherence behavior, that is free from limitations concerning patient participation and privacy concerns, could be the use of clinical vignettes (i.e. simulated practice situations). The systematic development of vignettes is based on clinical quality indicators, specifying the quality of delivered patient care, which are preferably retrieved from a combination of evidence-based guidelines and expert opinion (Campbell, Braspenning, Hutchinson, \& Marshall, 2003; Wollersheim et al., 2007), and are expected to result in vignettes with high content validity. In other fields, such as physiotherapy and suicide prevention, vignettes have already been used to successfully assess healthcare professionals' guideline adherence (de Beurs et al., 2015; Rutten et al., 2010). Applied to PNs' smoking cessation guideline adherence, a clinical vignette should prompt PNs to report the steps of the evidence-based guideline they would apply in response to a simulated situation of a smoking patient visiting their practice for support. It is not always relevant to apply all guideline steps during each consultation, as this depends on aspects like patient motivation (i.e. unmotivated, contemplating, motivated to quit) and the nature of the consultation (e.g. intake or follow-up) (Trimbos-instituut \& NHG, 2016). Hence, PNs need to receive several vignettes, differing on these aspects, to assess their adherence as described by the guideline. As clinical vignettes for smoking cessation guideline adherence do not yet exist, first systematic development of clinical quality indicators is needed based on the national guideline and expert opinion. Subsequently, quality indicators should be translated into vignettes and pilot tests should inform vignettes' reliability in practice, e.g. by comparing PNs' vignette answers to a small sample of recordings of consultations. Although the systematic development of quality indicators and clinical vignettes will be time-consuming (Wollersheim et al., 2007), this may result in a valid and reliable measure of guideline adherence which can easily be applied in practice.

\section{Theoretical reflections}

The I-Change Model (de Vries, 2017) has previously been used to successfully explain smoking cessation-related behavior of (practice) nurses (Bolman et al., 2002; Segaar, Willemsen, et al., 2007; Smit, de Vries, et al., 2013) and other healthcare professionals (Berndt et al., 2013; Hoving et al., 2007; Segaar, Bolman, et al., 2007). All studies reported on in this dissertation were therefore theoretically grounded in the I-Change Model, e.g. to develop measurement instruments for data collection and to develop the tailored content of the e-learning program.

Formative research results identified PNs' self-efficacy to use a guideline and PNs' perceived advantages of guideline use as important motivational determinants from the 
I-Change Model (de Ruijter, Smit, de Vries, \& Hoving, 2017). In the program's effect evaluation also multiple motivational determinants appeared important, as intervention group PNs made favorable progress concerning perceived (dis)advantages, self-efficacy, social modeling, social norms and social support. These findings corroborate the theoretical foundation of the I-Change Model, describing that a favorable motivation (and favorable motivational determinants) will have a positive effect on behavioral performance (de Vries, 2017). Moreover, also other determinants were found to be important, as PNs' engagement in smoking cessation counseling (i.e. time spent on counseling and counseling experience) was related to their guideline adherence. PNs' engagement in counseling is part of the I-Change Model's behavioral factors, which are described as preceding factors that can directly influence behavior but can also indirectly influence behavior through motivational determinants (de Vries, 2017). Yet, PNs' guideline adherence not only depends on preceding factors at PN level, but also depends on external determinants, like patients' motivation to quit smoking and the strength of the patient-PN relationship (Baummer-Carr \& Nicolau, 2017). These external determinants are not explicitly incorporated in the I-Change Model, but could affect PNs' perceived social support from patients, i.e. a factor that is included in the model. For instance, our data showed that a patient's low motivation to quit negatively influenced PNs' guideline adherence (de Ruijter, Smit, de Vries, Goossens, et al., 2017), possibly because PNs did not experience support to apply the guideline from these patients (de Vries, 2017). However, additional analyses did not find a relationship between PNs' perceived social support (both the overall support scale and support from patients specifically) and their guideline adherence in our trial. Furthermore, as we did not collect data on patients' cognitive determinants (e.g. patients' quit motivation) and the patient-PN relationship, additional research is required to establish if and how these factors influence PNs' guideline adherence, and whether these factors are in line with the theoretical foundation of the I-Change Model.

\section{Transferability of results to other professions or settings}

Our e-learning program was designed to promote PNs' adherence to the national evidence-based smoking cessation guideline in general practice. Yet, program usage might also be beneficial for other professionals involved in counseling smoking patients, in different healthcare settings and even in different countries, as many healthcare professionals also apply evidence-based smoking cessation guidelines. For instance, Dutch GPs and hospital staff are advised to use a similar or even the same guideline when counseling smoking patients (Chavannes et al., 2017; Trimbos-instituut \& NHG, 2016). Furthermore, although healthcare professionals in other countries are for instance trained to apply the American 5A approach (NICE, 2018) or British stop smoking services (Fiore et al., 2008), the content of these guidelines is largely similar to the steps described in the Dutch national guideline (Trimbos-instituut \& NHG, 2016). Hence, despite the variety in patients, settings and/or healthcare systems, our e-learning program is likely to provide relevant 
information and feedback to any healthcare professional counseling smokers to quit. Nevertheless, content and design features of our e-learning program were specifically based on formative research among Dutch PNs. It would therefore still be required to realize adaptations to program content and design to make it compatible to a new target population and/or healthcare system. This means that the core elements of the e-learning program, such as its evidence-based and tailored content, could largely remain similar, whereas more peripheral elements (e.g. related to the specific healthcare context, design features) should be changed to match the new target group's needs and preferences (Damschroder et al., 2009; Kok et al., 2016). For example, any change in context (e.g. a different patient population with specific smoking-related complaints) should be incorporated in both the tailored advice modules and the module with counseling information, based on a needs assessment among the new target group (Kok et al., 2016). This will improve the program's compatibility within the new healthcare setting and will make program engagement by healthcare professionals more likely (Yardley et al., 2016).

\section{Practical considerations}

\section{Implementation of the e-learning program in Dutch general practice}

Based on our findings concerning the (cost-)effectiveness of the e-learning program for PNs, it is worthwhile to pursue widespread usage of this theory- and evidence-based program by PNs throughout the Netherlands. However, to achieve national implementation of the e-learning program in Dutch general practices, we identified three implementation challenges that first need to be tackled: 1) PNs' program use in the RCT was only modest, 2) best practices for program implementation are yet unknown, and 3) a sustainable infrastructure for national implementation is not yet in place. To overcome these challenges, additional efforts are required.

First, insight needs to be obtained from PNs on how to further improve the program's usability, by optimizing the program's design and compatibility with general practice. As part of the RCT we already conducted a process evaluation (i.e. PN questionnaires, program log data, PN exit interviews), resulting in positive PN evaluations of both the overall program and the tailored advice modules (7.2/10). PNs especially appreciated that program content included information for both themselves (e.g. tailored feedback on their adherence) and for their patients (e.g. overview of effective smoking cessation aids). Still, PNs also had suggestions to improve the ease-of-navigation through the e-learning program and to reduce the length of text-based content, as application of the program was time consuming. As PNs' program use needs to improve for national program implementation to occur, it is important to collect additional recommendations from PNs on program adaptations that can further improve the e-learning program (e.g. using the nominal group technique to identify and rank improving alterations to the program (Delbecq 
\& Ven, 1971; Gallagher, Hares, Spencer, Bradshaw, \& Webb, 1993)), followed by usability tests to assess the program's improved usability (Jaspers, 2009; Nielsen \& Mack, 1994).

Secondly, strategies should be deployed to ensure PNs' program implementation in practice. A well-established strategy to promote change in healthcare organizations is to use peer-to-peer support via champions (Rogers, 2003; Valente \& Pumpuang, 2007), as they can promote skill-development and professionalization among their colleagues (Blake \& Chambers, 2012). Champions can play a vital role in introducing new programs into an organization and support a program's implementation (Dougherty \& Hardy, 1996; Shaw et al., 2012), as they can especially influence factors that promote implementation, such as building social support within an organization and mobilizing organizational resources (Blake \& Chambers, 2012; Shaw et al., 2012). By first identifying suitable PNs based on important role model characteristics, e.g. derived from the Motivational Theory of Role Modelling (e.g. sociability and similarity between role model and PN (Morgenroth, Ryan, \& Peters, 2015)) and then training them to become champions (i.e. function as role models and provide peer support), it could be a suitable solution to employ PN-champions to support PNs' program implementation.

Thirdly, a sustainable infrastructure should be realized to support national implementation of the e-learning program, as this process requires continuous effort and is not without costs. An important step for realizing a sustainable infrastructure is to make sure ownership of the e-learning program and peer champion strategy is transferred to one or more organizations that can bear implementation costs and that share the common goal to advance smoking cessation care in Dutch general practice (e.g. Trimbos, V\&VN, Quit Smoking Quality Register, Stop Smoking Partnership). Potential (co-)owners of the e-learning program and healthcare professionals involved in implementation of the program in general practice (e.g. PNs, PN-champions), should be involved in creating a business model for covering these implementation costs (van Gemert-Pijnen et al., 2011). By using the Business Model Canvas (Osterwalder \& Pigneur, 2013), the most practically and financially feasible model can be selected (van Limburg et al., 2015). Ultimately, a transfer of program ownership and the co-creation of a viable business model will aid in realizing national implementation of the e-learning program by PNs in Dutch general practice.

\section{General conclusions}

This dissertation described the systematic development and evaluation of a computertailored e-learning program for PNs to improve 1) PNs' adherence to the national, evidence-based smoking cessation guideline and 2) counseled smokers' abstinence rates. Based on the general discussion of the results of the studies presented, to-the-point conclusions and recommendations for research and practice are now provided. 
The studies reported on in this dissertation resulted in several novel findings, which could benefit research and practice. First, a new approach towards exploring PNs' guideline adherence, which we called step-based adherence, was used to gain detailed insight in PNs' smoking cessation counseling and the determinants of their adherence to individual guideline steps. This approach should be applied in other care areas as well to prove its usefulness for understanding guideline adherence behavior. Secondly, we demonstrated how systematic research among the target population resulted in developing the first tailored e-learning program for PNs. This project contributes to existing knowledge on developing evidence-based, digital behavior change programs, which is especially relevant as the demand for eHealth innovations is increasing in research and practice. Thirdly, our RCT illustrated that providing PNs with program access was (cost-)effective for improving their adherence to the national smoking cessation guideline. These are the first findings demonstrating that investing in e-learning for PNs is a cost-effective strategy to improve the quality of patient care in general practice.

Based on the barriers and facilitating factors that we experienced while conducting the research reported on in this dissertation, several 'lessons learned' are presented. Firstly, the efficiency of participant recruitment, in particular the recruitment of smoking patients, could benefit from investing in more personal contact between the research team and patients, and detailed analyses of recruitment strategies via healthcare professionals. Secondly, as PNs used the e-learning program less frequently than expected, we propose that additional evidence-based personalization efforts, such as applying modetailoring and providing tailored reminders, should be applied to improve PNs' program use.

Based on this dissertation's findings, several gaps in scientific knowledge were identified that would benefit from additional research efforts. First, a lack of a reliable assessment to measure guideline adherence was identified. We intended to create a triangulated adherence measure, but difficulties with collecting patient data and recording consultations interfered with this. Future research should therefore focus on developing and testing a measure of guideline adherence that is free from these limitations, such as clinical vignettes. Secondly, more research is needed concerning factors directly influencing the quality of PNs' counseling and indirectly influencing patients' quit success, including 1) the strength of the patient-PN relationship, 2) PNs' motivational interviewing skills, and 3) patients' motivation to quit smoking. Finally, as the e-learning program was (cost-)effective for improving PNs' smoking cessation guideline adherence, we recommend national program implementation to make it widely available for PNs in Dutch general practice. Yet, additional research is required to overcome identified implementation challenges and to ensure application of systematic, evidence-based implementation strategies. 
Valorization Addendum 
The aim of this research project was to provide detailed insight in the current smoking cessation counseling practices of practice nurses in Dutch general practice and to accordingly develop a tailored e-learning program to further improve their counseling practices. In chapter 2 and 3 we elaborated on practice nurses' application of the national smoking cessation guideline and confirmed that there is room for improvement. As complete guideline adherence is known to improve the quality and effectiveness of smoking cessation care (Rice, Heath, Livingstone-Banks, \& Hartmann-Boyce, 2017; Stead et al., 2013), the relevance of this project emanates from this need for improving practice nurses' adherence to the guideline. In chapter 5 and 6, we demonstrated that the developed elearning program was (cost-)effective to improve practice nurses' guideline adherence, meaning that the program can promote the quality and effectiveness of smoking cessation care. The relevance of the project results goes beyond application by practice nurses only and will hence be discussed in a broader context as well, including impact on other healthcare professionals, decision makers and society. Finally, dissemination of the project's results, more specifically widespread implementation of the e-learning program by healthcare professionals, will be discussed.

\section{Relevance for smoking cessation care}

Based on the formative research, reported on in chapter 2 and 3, we developed an evidence-based adherence support program for practice nurses. As primary care professionals have to deal with increasing care demands, and hence increasing time constraints (Halcomb et al., 2015; van Rossem et al., 2015), this program can offer an efficient, (cost)effective approach to improve the quality of the smoking cessation care they provide. Providing practice nurses with an effective e-learning program, with 24/7 online accessibility, may reduce their need to participate in more expensive and time consuming faceto-face workshops or training programs. To increase the program's reach and visibility, the e-learning program should be registered in the national database with evidencebased interventions (i.e. www.loketgezondleven.nl), which is managed by the Dutch National Institute for Public Health and the Environment (RIVM). Furthermore, program impact also depends on implementation by its end-users (Glasgow, Lichtenstein, \& Marcus, 2003). Therefore, to stimulate practice nurses to implement the program, additional steps will be required to ensure that practice nurses receive the appropriate recognition (i.e. accreditation points) for engaging with the e-learning program, as this was reported to be a facilitating factor for repeated program use. This means that certification for the e-learning program needs to be obtained from national organizations for primary care professionals (e.g. Verpleegkundigen \& Verzorgenden Nederland (V\&VN), Nederlandse Vereniging van Praktijkondersteuners (NVVPO)), which will enable practice nurse to apply for accreditation points when they intent to use the e-learning program. 
Many healthcare professionals (e.g. hospital nurses, physicians, dentists, midwives), in the Netherlands and also abroad, apply evidence-based guidelines when counseling their patients in general, but also specifically concerning smoking cessation counseling. This means that usage of the e-learning program might also be beneficial for a broader range of healthcare professionals. Although these professionals operate in a variety of settings and patient groups, our e-learning program - being able to provide individually tailored content - is likely to provide relevant information and feedback to any professional that engages in counseling with smoking patients. To ensure the program's compatibility with a different context, it is important to conduct a needs assessment among the new target group (Kok et al., 2016) and make changes to the program accordingly. Moreover, to make the program compatible to a different context that a healthcare professional (e.g. a midwife) works in when engaging in counseling with a smoking patient, program changes are required in both the tailored advice modules (e.g. advice tailored to the content of the evidence-based smoking cessation guideline for midwives, the $\mathrm{V}$ MIS) and the module with counseling information (e.g. practical information for pregnant women about the effects of smoking on their (unborn) baby). Besides the compatibility of program content with a new healthcare setting, also structural changes might be required to enhance chances of the program being compatible with existing (digital) information structures. With respect to structural compatibility, it is important to explore technological developments in a healthcare setting (e.g. type of digital information system used, availability of an existing eHealth platform) and to take steps to optimize the program's compatibility with such technologies. Hence, resulting from content adaptation and continuous structural development of the e-learning program, an improved version of the program should be created (i.e. version 2.0). The updated program will then need to be tested (i.e. pilot-tests, usability tests) by the new target population to determine chances of adequate program implementation in practice. Such an evidence-based approach, based on stakeholder involvement, will be essential to ensure relevance (and effectiveness) of the e-learning program in other healthcare settings besides Dutch general practice.

\section{Relevance for society}

In Dutch general practice, a shift has taken place towards more care responsibilities, including smoking cessation counseling, being provided by a practice nurses instead of the general practitioner (Dierick-van Daele, Metsemakers, Derckx, Spreeuwenberg, \& Vrijhoef, 2009; van Hassel, Batenburg, \& van der Velden, 2016). Practice nurse-led care and general practitioner-led care do not differ in terms of quality and lead to comparable scores on patient satisfaction, while practice nurses' care is associated with less costs (Dierick-van Daele et al., 2009; Swan, Ferguson, Chang, Larson, \& Smaldone, 2015; van 
Rossem et al., 2017). This illustrates that practice nurse-led care can be a cost-saving alternative for general practitioner-led care. This means that an intervention that can improve the quality of practice nurses' care, such as our e-learning program, will improve the likelihood that practice nurse-led care in general practice is not only cost-saving but also more cost-effective compared to general practitioner-led care. This is relevant for decision makers in healthcare, as they rely on evidence about the costs and effects of care to make decisions about the allocation of financial resources (Evers, Wolfs, \& Van Heugten, 2010). The project results are relevant, as they provide insight in the costs, on a societal level, that are associated with evidence-based smoking cessation care by practice nurses. The economic evaluation of our e-learning program revealed that the program was a cost-effective addition to practice nurses' usual smoking cessation care. This increases the likelihood that practice nurses will outperform general practitioners in terms of providing high-quality smoking cessation care. As we know that costs associated with practice nurse-led care will be lower or equal compared to general practitioner-led care, this increases the cost-effectiveness of care by practice nurses. In light of the fact that practice nurses are increasingly responsible for smoking cessation care, further improvement of their cost-effective care will have financial benefits on a societal level. More importantly, effective smoking cessation care from practice nurses will benefit the overall health on a societal level, as smoking patients receive high quality care likely resulting in more smoking abstinence.

Within society, specific healthcare organizations might benefit from the project results. In particular, national nursing organizations (e.g. V\&VN, NVvPO) or national stop smoking organizations (e.g. Kwaliteitsregister Stoppen met Roken, Alliantie Nederland Rookvrij) benefit from the project as they are interested in ways to improve the provision of evidence-based (smoking cessation) care in Dutch healthcare. They might be interested in the e-learning program as a means to promote continuous professionalization of healthcare professionals. Moreover, these national healthcare organizations have members working in all regions in the Netherlands, meaning that the online and tailored nature of the e-learning program will be particularly important to efficiently support professionals throughout the country.

Furthermore, the e-learning program is of interest for educational institutes that are responsible for training healthcare professionals to provide evidence-based care. In the Netherlands, one could think of national organizations that offer professional education, such as a practice nurse master curriculum (e.g. offered by various institutes for higher education) or a smoking cessation refresher course (e.g. offered by the Netherlands School of Public and Occupational Health (NSPOH)). Our e-learning program could, for instance, be offered as a complementary element in addition to the face-to-face training sessions offered by such educational institutes, or could even be offered as a stand-alone educational activity concerning the application of an evidence-based smoking cessation 
guideline by an educational institute. By providing access to the e-learning program in collaboration with educational institutes, healthcare professionals might save time and money as a result of reduced travel and time costs. Educational institutes might also benefit from expanding their e-learning curriculum, as they can likely attract a wider range of professionals and hence expand their reach and generate more revenue.

\section{Dissemination}

\section{Co-creation with relevant stakeholders}

In light of the positive results concerning the (cost-)effectiveness of the e-learning program we consider it worthwhile to pursue widespread implementation of the program in general practices in the Netherlands. To achieve this, additional implementation efforts are required, including 1 ) optimization of the e-learning program's usability to promote its usage by practice nurses, 2) the development of an evidence-based peer support implementation strategy based on employing champions to function as role models, and 3 ) the transfer of ownership of the program and peer support strategy, and the creation of a viable business model. Nowadays it is particularly recommended to invest in interdisciplinary collaboration and end-user involvement (i.e. co-creation) when aiming for intervention development and implementation (de Boer \& Bosman, 2018; Smeets \& ZijlstraVlasveld, 2016; Swinkels et al., 2018). Hence, for the widespread implementation of our e-learning program, potential owners of the e-learning program (e.g. NSPOH, Trimbos, V\&VN, Quit Smoking Quality Register, Stop Smoking Partnership) and healthcare professionals involved in implementation of the program in general practice (e.g. practice nurses, champions, general practitioners) should be involved to guarantee funding for and co-creation of the implementation strategy.

Realizing interdisciplinary collaboration and co-creation are known to play a vital role in the use of digital interventions (van Gemert-Pijnen et al., 2011; Yardley et al., 2016), and were hence an important part of the development and evaluation of the e-learning program is the present project. Yet, these aspects remain essential during the course of an implementation project, as well as during the period after the implementation project ends. Therefore, to optimize chances for continued implementation of the e-learning program after the ending of the project, it is essential to already establish a stakeholder advisory board (including potential program owners and relevant healthcare professionals) early on in an implementation project. Stakeholders representing a national organization (e.g. Trimbos, V\&VN) can facilitate dissemination of and support for continued program implementation on a large scale within their professional network. Moreover, a stakeholder like the NSPOH will be especially important, because of its extensive experience in developing and offering training courses for healthcare professionals including practice nurses. Also, this organization is perceived as reliable and trustworthy by healthcare professionals, meaning that involvement of the NSPOH in the implementation 
strategy increases the likelihood that practice nurses have a positive attitude towards implementation of the e-learning program. Through co-creation with relevant healthcare professionals, an implementation plan will better match facilitating factors and barriers related to the general practice setting and health professionals' daily practice; thereby increasing the plan's level of compatibility with current practices. This could even support healthcare groups', general practices' and general practice staff's evaluation of their own organization or daily practice beyond the duration of this project and facilitate implementation of the e-learning program and peer champion strategy in new healthcare groups.

\section{Budgeting models and marketing mix}

As national implementation of the e-learning program is not without costs, it is also important to discuss possible budgeting models with members from the stakeholder advisory board. Costs associated with national program implementation include costs for continued program maintenance and technical support; personnel costs (i.e. a project coordinator); communication costs (continuous recruitment and stakeholder contact); costs for continued accreditation of the e-learning program; and costs associated with the implementation strategy (e.g. training practice nurse-champions). The feasibility and success of an implementation project will for a large extent depend on the willingness and ability of important stakeholders to bear such implementation costs. A comparison of different budgeting models (e.g. health insurer budget or training budget of a healthcare group), based on the Business Model Canvas (Osterwalder \& Pigneur, 2013), can be used to support stakeholders to make an informed decision about a financially viable implementation plan (van Limburg, Wentzel, Sanderman, \& van Gemert-Pijnen, 2015). During meetings of the stakeholder advisory board, the sustainability of implementation costs can be discussed in order to reach consensus about an appropriate budgeting model. Ultimately, the new owner(s) of the e-learning program must be willing to take responsibility for bearing costs associated with sustainable program implementation.

Nowadays, in support of implementation activities additional efforts are often required to increase chances that a carefully planned implementation project will achieve its intended reach and impact. Therefore, it is suggested to systematically incorporate theory-based marketing strategies in the design and evaluation of health interventions. Especially the principles of the marketing mix (4 P's: product, price, place, promotion) can facilitate change among a target audience (Luca \& Suggs, 2013). In line with what has been already described, also aspects related to the 4P's should be explored through cocreation with the target population. This will ensure that the marketing mix is in line with needs and expectations of the target population. Moreover, concerning aspects like product and price, it might be important to ensure that the e-learning program has a highquality design and is associated with a reliable organization like the NSPOH (i.e. product 
characteristics), and that the program owner offers the e-learning program via a budgeting model that ensures minimal financial investment from individual practice nurses (i.e. price characteristics). Concerning place, it seems worthwhile to invest in distribution of the e-learning program via various national organizations, while it might be important to explore whether direct distribution (i.e. originating from the owner) or distribution via intermediate links or agents is most feasible in practice. Finally, a detailed promotion strategy should be developed to increase the owner's brand image (e.g. obtain sponsor payment from a trustworthy national organization) and to reach a large target audience through targeted advertisements. Investing in developing a co-created marketing strategy warrants more attention in future implementation plans of health promotion and prevention campaigns, as it may increase the public health impact of such interventions.

Based on such evidence-based implementation efforts it is expected that both the reach and use of the e-learning program by practice nurses would increase. This will in turn lead to bigger program impact, both regarding practice nurses' adherence to the national smoking cessation guideline as well as regarding an indirect program impact on the level of smoking abstinence of counseled patients in Dutch general practice. 

References 
Abrahamson, K. A., Fox, R. L., \& Doebbeling, B. N. (2012). Facilitators and barriers to clinical practice guideline use among nurses. Am J Nurs, 112(7), 26-35; quiz 46,36. doi:10.1097/01.NAJ.0000415957.46932.bf

Adams, A. S., Soumerai, S. B., Lomas, J., \& Ross-Degnan, D. (1999). Evidence of self-report bias in assessing adherence to guidelines. Int J Qual Health Care, 11(3), 187-192.

Agboola, S., McNeill, A., Coleman, T., \& Bee, J. L. (2010). A systematic review of the effectiveness of smoking relapse prevention interventions for abstinent smokers. Addiction, 105(8), 1362-1380. doi:doi:10.1111/j.1360-0443.2010.02996.x

Ahmed, B., Dannhauser, T., \& Philip, N. (2018). A systematic review of reviews to identify key research opportunities within the field of eHealth implementation. J Telemed Telecare, $1357633 \times 18768601$. doi:10.1177/1357633×18768601

Ajzen, I. (1985). From intentions to actions: A theory of planned behavior. In J. Kuhl \& J. Beckman (Eds.), Actioncontrol: From cognition to behavior Heidelberg: Springer.

Al-Janabi, H., Flynn, T. N., \& Coast, J. (2012). Development of a self-report measure of capability wellbeing for adults: the ICECAP-A. Qual Life Res, 21(1), 167-176. doi:10.1007/s11136-011-9927-2

Ariens, L. F., Schussler-Raymakers, F. M., Frima, C., Flinterman, A., Hamminga, E., Arents, B. W., . . van OsMedendorp, H. (2017). Barriers and Facilitators to eHealth Use in Daily Practice: Perspectives of Patients and Professionals in Dermatology. J Med Internet Res, 19(9), e300. doi:10.2196/jmir.7512

Asfar, T., Lee, D. J., Lam, B. L., Murchison, A. P., Mayro, E. L., Owsley, C., . . Saaddine, J. (2017). Evaluation of a Web-Based Training in Smoking Cessation Counseling Targeting U.S. Eye-Care Professionals. Health Educ Behav, 1090198117709883. doi:10.1177/1090198117709883

Avis, J. L., van Mierlo, T., Fournier, R., \& Ball, G. D. (2015). Lessons Learned From Using Focus Groups to Refine Digital Interventions. JMIR Res Protoc, 4(3), e95. doi:10.2196/resprot.4404

Bader, P., McDonald, P., \& Selby, P. (2009). An algorithm for tailoring pharmacotherapy for smoking cessation: results from a Delphi panel of international experts. Tobacco Control, 18(1), 34-42. doi:10.1136/tc.2008.025635

Baker, R., Camosso-Stefinovic, J., Gillies, C., Shaw, E. J., Cheater, F., Flottorp, S., . . Jager, C. (2015). Tailored interventions to address determinants of practice. Cochrane Database Syst Rev(4), Cd005470. doi:10.1002/14651858.CD005470.pub3

Bandura, A. (1986). Social foundations of thought and action: A social-cognitive theory. Englewood Cliffs, NJ: Prentice Hall.

Batenburg, R. (2016). Praktijkovername in de eerste lijn: huisartsen, tandartsen en apothekers over hun overnamewensen als praktijkhouder of praktijkzoeker. Retrieved from Utrecht:

Baummer-Carr, A., \& Nicolau, D. P. (2017). The challenges of patient satisfaction: influencing factors and the patient - provider relationship in the United States. Expert Review of Anti-infective Therapy, 15(10), 955962. doi:10.1080/14787210.2017.1378097

Bennett, G. G., \& Glasgow, R. E. (2009). The delivery of public health interventions via the Internet: actualizing their potential. Annu Rev Public Health, 30, 273-292. doi:10.1146/annurev.publhealth.031308.100235

Berndt, N., Bolman, C., Froelicher, E. S., Mudde, A., Candel, M., de Vries, H., \& Lechner, L. (2014). Effectiveness of a telephone delivered and a face-to-face delivered counseling intervention for smoking cessation in patients with coronary heart disease: a 6-month follow-up. I Behav Med, 37(4), 709-724. doi:10.1007/s10865-013-9522-9

Berndt, N., Bolman, C., Lechner, L., Max, W., Mudde, A., de Vries, H., \& Evers, S. (2016). Economic evaluation of a telephone- and face-to-face-delivered counseling intervention for smoking cessation in patients with coronary heart disease. Eur J Health Econ, 17(3), 269-285. doi:10.1007/s10198-015-0677-x

Berndt, N. C., Bolman, C., de Vries, H., Segaar, D., van Boven, I., \& Lechner, L. (2013). Smoking cessation treatment practices: recommendations for improved adoption on cardiology wards. J Cardiovasc Nurs, 28(1), 35-47. doi:10.1097/JCN.0b013e318231f424

Blake, H., \& Chambers, D. (2012). Supporting nurse health champions. Health Education Journal, 71(2), 205210. doi:doi:10.1177/0017896910396767 
Bolman, C., de Vries, H., \& Mesters, I. (2002). Factors determining cardiac nurses' intentions to continue using a smoking cessation protocol. Heart \& Lung: The Journal of Acute and Critical Care, 31(1), 15-24. doi:http://dx.doi.org/10.1067/mhl.2002.119834

Bolman, C., Eggers, S. M., van Osch, L., Te Poel, F., Candel, M., \& de Vries, H. (2015). Is Action Planning Helpful for Smoking Cessation? Assessing the Effects of Action Planning in a Web-Based Computer-Tailored Intervention. Subst Use Misuse, 1-12. doi:10.3109/10826084.2014.977397

Bond, S. E., Crowther, S. P., Adhikari, S., Chubaty, A. J., Yu, P., Borchard, J. P., . . Miyakis, S. (2017). Design and Implementation of a Novel Web-Based E-Learning Tool for Education of Health Professionals on the Antibiotic Vancomycin. J Med Internet Res, 19(3), e93. doi:10.2196/jmir.6971

Bourgeault, I., Dingwall, R., \& Vries, R. d. (2010). The SAGE Handbook of Qualitative Methods in Health Research: SAGE Publications Ltd.

Brandes, K., \& van Weert, J. C. M. (2017). Implementing consultation audio-recordings and question prompt lists into routine cancer care: How can we address healthcare providers' barriers? Patient Educ Couns, 100(6), 1029-1030. doi:https://doi.org/10.1016/j.pec.2017.04.002

Brouwer, W., Kroeze, W., Crutzen, R., de Nooijer, J., de Vries, N. K., Brug, J., \& Oenema, A. (2011). Which intervention characteristics are related to more exposure to internet-delivered healthy lifestyle promotion interventions? A systematic review. J Med Internet Res, 13(1), e2. doi:10.2196/jmir.1639

Bundy, C. (2004). Changing behaviour: using motivational interviewing techniques. J R Soc Med, 97 Suppl 44, 43-47.

Campbell, J. L., Quincy, C., Osserman, J., \& Pedersen, O. K. (2013). Coding In-depth Semistructured Interviews: Problems of Unitization and Intercoder Reliability and Agreement. Sociological Methods \& Research, 42(3), 294-320. doi:10.1177/0049124113500475

Campbell, M., Fitzpatrick, R., Haines, A., Kinmonth, A. L., Sandercock, P., Spiegelhalter, D., \& Tyrer, P. (2000). Framework for design and evaluation of complex interventions to improve health. BMJ, 321(7262), 694-696.

Campbell, S. M., Braspenning, J., Hutchinson, A., \& Marshall, M. N. (2003). Research methods used in developing and applying quality indicators in primary care. BMJ, 326(7393), 816-819. doi:10.1136/bmj.326. 7393.816

Candel, M. J., \& Van Breukelen, G. J. (2010). Sample size adjustments for varying cluster sizes in cluster randomized trials with binary outcomes analyzed with second-order PQL mixed logistic regression. Stat Med, 29(14), 1488-1501. doi:10.1002/sim.3857

Cantor, S. B., Deshmukh, A. A., Luca, N. S., Nogueras-Gonzalez, G. M., Rajan, T., \& Prokhorov, A. V. (2015). Costeffectiveness analysis of smoking-cessation counseling training for physicians and pharmacists. Addict Behav, 45, 79-86. doi:10.1016/j.addbeh.2015.01.004

Cassista, J., Payne-Gagnon, J., Martel, B., \& Gagnon, M. P. (2014). Applying Theory to Understand and Modify Nurse Intention to Adhere to Recommendations regarding the Use of Filter Needles: An Intervention Mapping Approach. Nurs Res Pract, 2014, 356153. doi:10.1155/2014/356153

Centraal Bureau voor de Statistiek. (2017). Consumentenprijzen; prijsindex $2015=100$. Retrieved from http://statline.cbs.nl/Statweb/publication/?DM=SLNL\&PA=83131ned\&D1=0-6\&D2=0\&D3=(I-39)-I\&VW=T

Chavannes, N., Drenthen, T., Wind, L., Van Avendonk, M., Van den Donk, M., \& Verduijn, M. (2017). NHG-Behandelrichtlijn Stoppen met roken. Retrieved from NHG Nederlands Huisartsen Genootschap. Utrecht, Nederland:

Chirikos, T. N., Herzog, T. A., Meade, C. D., Webb, M. S., \& Brandon, T. H. (2004). Cost-effectiveness analysis of a complementary health intervention: The case of smoking relapse prevention. International Journal of Technology Assessment in Health Care, 20(4), 475-480. doi:10.1017/S0266462304001382

Civljak, M., Stead, L. F., Hartmann-Boyce, J., Sheikh, A., \& Car, J. (2013). Internet-based interventions for smoking cessation. Cochrane Database Syst Rev, 7, CD007078. doi:10.1002/14651858.CD007078.pub4

Cohen, J. E. (1988). Statistical power analysis for the behavioral sciences (2nd edition ed.). Hillsdale, NJ: Lawrence Erlbaum Associates.

Coleman, T., Agboola, S., Leonardi-Bee, J., Taylor, M., McEwen, A., \& McNeill, A. (2010). Relapse prevention in UK Stop Smoking Services: current practice, systematic reviews of effectiveness and cost-effectiveness analysis. Health Technol Assess, 14(49), 1-152, iii-iv. doi:10.3310/hta14490

Collins, S. E., Witkiewitz, K., Kirouac, M., \& Marlatt, G. A. (2010). Preventing Relapse Following Smoking Cessation. Current cardiovascular risk reports, 4(6), 421-428. doi:10.1007/s12170-010-0124-6 
Cook, D. A., Levinson, A. J., Garside, S., Dupras, D. M., Erwin, P. J., \& Montori, V. M. (2010). Instructional design variations in internet-based learning for health professions education: a systematic review and meta-analysis. Acad Med, 85(5), 909-922. doi:10.1097/ACM.0b013e3181d6c319

Coolbrandt, A., Wildiers, H., Laenen, A., Aertgeerts, B., Dierckx de Casterle, B., van Achterberg, T., \& Milisen, K. (2018). A Nursing Intervention for Reducing Symptom Burden During Chemotherapy. Oncol Nurs Forum, 45(1), 115-128. doi:10.1188/18.onf.115-128

Copeland, L., McNamara, R., Kelson, M., \& Simpson, S. (2015). Mechanisms of change within motivational interviewing in relation to health behaviors outcomes: A systematic review. Patient Educ Couns, 98(4), 401411. doi:http://dx.doi.org/10.1016/j.pec.2014.11.022

Damschroder, L. J., Aron, D. C., Keith, R. E., Kirsh, S. R., Alexander, J. A., \& Lowery, J. C. (2009). Fostering implementation of health services research findings into practice: a consolidated framework for advancing implementation science. Implement Sci, 4, 50. doi:10.1186/1748-5908-4-50

Davis, R., Campbell, R., Hildon, Z., Hobbs, L., \& Michie, S. (2015). Theories of behaviour and behaviour change across the social and behavioural sciences: a scoping review. Health Psychol Rev, 9(3), 323-344. doi:10.1080/17437199.2014.941722

de Beurs, D. P., de Groot, M. H., de Keijser, J., Mokkenstorm, J., van Duijn, E., de Winter, R. F. P., \& Kerkhof, A. J. F. M. (2015). The effect of an e-learning supported Train-the-Trainer programme on implementation of suicide guidelines in mental health care. Journal of Affective Disorders, 175, 446-453. doi:http://dx.doi.org/10.1016/j.jad.2015.01.046

de Boer, J., \& Bosman, Q. (2018). Toekomst digitalisering eerstelijnszorg Huisartsen. Nictiz, Den Haag, Nederland. de Hoog, N., Bolman, C., Berndt, N., Kers, E., Mudde, A., de Vries, H., \& Lechner, L. (2016). Smoking cessation in cardiac patients: the influence of action plans, coping plans and self-efficacy on quitting smoking. Health Educ Res. doi:10.1093/her/cyv100

de Leeuw, J., Prins, J. B., Uitterhoeve, R., Merkx, M. A., Marres, H. A., \& van Achterberg, T. (2014). Nurse-patient communication in follow-up consultations after head and neck cancer treatment. Cancer Nurs, 37(2), E19. doi:10.1097/NCC.0b013e318288d3f3

de Ruijter, D., Candel, M., Smit, E. S., de Vries, H., \& Hoving, C. (2018). The Effectiveness of a Computer-Tailored E-Learning Program for Practice Nurses to Improve Their Adherence to Smoking Cessation Counseling Guidelines: Randomized Controlled Trial. J Med Internet Res, 20(5), e193. doi:10.2196/ jmir.9276

de Ruijter, D., Smit, E. S., de Vries, H., Goossens, L., \& Hoving, C. (2017). Understanding Dutch practice nurses' adherence to evidence-based smoking cessation guidelines and their needs for web-based adherence support: results from semistructured interviews. BMJ Open, 7(3), e014154. doi:10.1136/bmjopen-2016014154

de Ruijter, D., Smit, E. S., De Vries, H., \& Hoving, C. STER studie. Retrieved from www.sterstudie.nl Archived at WebCite on 26-7-2016 http://www.webcitation.org/6jHiDBycb

de Ruijter, D., Smit, E. S., de Vries, H., \& Hoving, C. (2016). Web-based computer-tailoring for practice nurses aimed to improve smoking cessation guideline adherence: A study protocol for a randomized controlled effectiveness trial. Contemp Clin Trials, 48, 125-132. doi:10.1016/j.cct.2016.04.007

de Ruijter, D., Smit, E. S., de Vries, H., \& Hoving, C. (2017). Dutch practice nurses' adherence to evidence-based smoking cessation treatment guidelines. Fam Pract. doi:10.1093/fampra/cmx039

de Vries, H. (2017). An Integrated Approach for Understanding Health Behavior; The I-Change Model as an Example. Psychology and Behavioral Science International Journal, 2(2), 1-4. doi:10.19080/ PBSIJ.2017.02.555585.

de Vries, H., \& Brug, J. (1999). Computer-tailored interventions motivating people to adopt health promoting behaviours: introduction to a new approach. Patient Educ Couns, 36(2), 99-105.

de Vries, H., Eggers, S. M., \& Bolman, C. (2013). The role of action planning and plan enactment for smoking cessation. BMC Public Health, 13, 393. doi:10.1186/1471-2458-13-393

de Vries, H., Mudde, A., Leijs, I., Charlton, A., Vartiainen, E., Buijs, G., . . Kremers, S. (2003). The European Smoking Prevention Framework Approach (EFSA): an example of integral prevention. Health Educ Res, 18(5), 611-626.

Deci, E. L., \& Ryan, R. M. (1985). Intrinsic motivation and self-determination in human behavior. New York: Plenum. 
Delbecq, A. L., \& Ven, A. H. V. d. (1971). A Group Process Model for Problem Identification and Program Planning. The Journal of Applied Behavioral Science, 7(4), 466-492. doi:10.1177/002188637100700404

Dierick-van Daele, A. T., Metsemakers, J. F., Derckx, E. W., Spreeuwenberg, C., \& Vrijhoef, H. J. (2009). Nurse practitioners substituting for general practitioners: randomized controlled trial. J Adv Nurs, 65(2), 391401. doi:10.1111/j.1365-2648.2008.04888.x

Dougherty, D., \& Hardy, C. (1996). Sustained Product Innovation in Large, Mature Organizations: Overcoming Innovation-to-Organization Problems. ACAD MANAGE J, 39(5), 1120-1153.

Duffy, M. E. (2002). Methodological Issues In Web-based Research. Journal of Nursing Scholarship, 34(1), 8388. doi:doi:10.1111/j.1547-5069.2002.00083.x

Ebbert, J. O., Hays, J. T., \& Hurt, R. D. (2010). Combination pharmacotherapy for stopping smoking: what advantages does it offer? Drugs, 70(6), 643-650. doi:10.2165/11536100-000000000-00000

Edwards, C., Staniszweska, S., \& Crichton, N. (2004). Investigation of the ways in which patients' reports of their satisfaction with healthcare are constructed. Sociol Health IIIn, 26(2), 159-183. doi:10.1111/j.14679566.2004.00385.x

Efraimsson, E. O., Fossum, B., Ehrenberg, A., Larsson, K., \& Klang, B. (2012). Use of motivational interviewing in smoking cessation at nurse-led chronic obstructive pulmonary disease clinics. J Adv Nurs, 68(4), 767-782. doi:10.1111/j.1365-2648.2011.05766.x

Efraimsson, E. O., Klang, B., Ehrenberg, A., Larsson, K., Fossum, B., \& Olai, L. (2015). Nurses' and patients' communication in smoking cessation at nurse-led COPD clinics in primary health care. Eur Clin Respir J, 2, 27915. doi:10.3402/ecrj.v2.27915

Ekeland, A. G., Bowes, A., \& Flottorp, S. (2010). Effectiveness of telemedicine: a systematic review of reviews. Int J Med Inform, 79(11), 736-771. doi:10.1016/j.ijmedinf.2010.08.006

Elbert, N. J., van Os-Medendorp, H., van Renselaar, W., Ekeland, A. G., Hakkaart-van Roijen, L., Raat, H., . . . Pasmans, S. G. (2014). Effectiveness and Cost-Effectiveness of eHealth Interventions in Somatic Diseases: A Systematic Review of Systematic Reviews and Meta-Analyses. J Med Internet Res, 16(4), e110. doi:10.2196/jmir.2790

Elfeddali, I., Bolman, C., Candel, M. J., Wiers, R. W., \& de Vries, H. (2012). Preventing smoking relapse via Webbased computer-tailored feedback: a randomized controlled trial. J Med Internet Res, 14(4), e109. doi:10.2196/jmir.2057

Evers, S., Wolfs, C., \& Van Heugten, C. (2010). Economische evaluatie van neurospychologische behandeling. In R. Ponds, C. van Heugten, L. Fasotti, \& E. Wekking (Eds.), Neuropsychologische Behandeling. Amsterdam: Boom Uitgeverij.

Eysenbach, G. (2005). The law of attrition. J Med Internet Res, 7(1), e11. doi:10.2196/jmir.7.1.e11

Eysenbach, G. (2011). CONSORT-EHEALTH: Improving and Standardizing Evaluation Reports of Web-based and Mobile Health Interventions. J Med Internet Res, 13(4), e126. doi:10.2196/jmir.1923

Fenwick, E., O'Brien, B. J., \& Briggs, A. (2004). Cost-effectiveness acceptability curves - facts, fallacies and frequently asked questions. Health Econ, 13(5), 405-415. doi:10.1002/hec.903

Fiore, M., Jaén, C., Baker, T., Bailey, W., Benowitz, N., Curry, S., ... Wewers, M. (2008). Treating Tobacco Use and Dependence: 2008 Update. Retrieved from Department of Health and Human Services. Rockville, MD: U.S.:

Fishbein, M., \& Yzer, M. C. (2003). Using Theory to Design Effective Health Behavior Interventions. Communication Theory, 13(2), 164-183. doi:10.1111/j.1468-2885.2003.tb00287.x

Flay, B. R. (1986). Efficacy and effectiveness trials (and other phases of research) in the development of health promotion programs. Prev Med, 15(5), 451-474.

Fleuren, M., Wiefferink, K., \& Paulussen, T. (2004). Determinants of innovation within health care organizations: literature review and Delphi study. Int J Qual Health Care, 16(2), 107-123. doi:10.1093/intqhc/ mzh030

Fontaine, G., Cossette, S., Heppell, S., Boyer, L., Mailhot, T., Simard, M.-J., \& Tanguay, J.-F. (2016). Evaluation of a Web-Based E-Learning Platform for Brief Motivational Interviewing by Nurses in Cardiovascular Care: A Pilot Study. J Med Internet Res, 18(8), e224. doi:10.2196/jmir.6298

Freund, T., Everett, C., Griffiths, P., Hudon, C., Naccarella, L., \& Laurant, M. (2015). Skill mix, roles and remuneration in the primary care workforce: Who are the healthcare professionals in the primary care teams across the world? Int J Nurs Stud, 52(3), 727-743. doi:10.1016/j.ijnurstu.2014.11.014 
Friederichs, S. A., Oenema, A., Bolman, C., \& Lechner, L. (2015). Long term effects of self-determination theory and motivational interviewing in a web-based physical activity intervention: randomized controlled trial. Int J Behav Nutr Phys Act, 12, 101. doi:10.1186/s12966-015-0262-9

Fry, J. P., \& Neff, R. A. (2009). Periodic Prompts and Reminders in Health Promotion and Health Behavior Interventions: Systematic Review. J Med Internet Res, 11(2), e16. doi:10.2196/jmir.1138

Gale, N. K., Heath, G., Cameron, E., Rashid, S., \& Redwood, S. (2013). Using the framework method for the analysis of qualitative data in multi-disciplinary health research. BMC Med Res Methodol, 13, 117. doi:10.1186/1471-2288-13-117

Gallagher, M., Hares, T. I. M., Spencer, J., Bradshaw, C., \& Webb, I. A. N. (1993). The Nominal Group Technique: A Research Tool for General Practice? Fam Pract, 10(1), 76-81. doi:10.1093/fampra/10.1.76

Geraghty, A. W., Torres, L. D., Leykin, Y., Perez-Stable, E. J., \& Munoz, R. F. (2013). Understanding attrition from international internet health interventions: a step towards global eHealth. Health Promot Int, 28(3), 442452. doi:10.1093/heapro/das029

Glanz, K., \& Bishop, D. B. (2010). The role of behavioral science theory in development and implementation of public health interventions. Annu Rev Public Health, 31, 399-418. doi:10.1146/annurev.publhealth. 012809.103604

Glasgow, R. E., Lichtenstein, E., \& Marcus, A. C. (2003). Why don't we see more translation of health promotion research to practice? Rethinking the efficacy-to-effectiveness transition. Am J Public Health, 93(8), 1261-1267.

Glasgow, R. E., Vogt, T. M., \& Boles, S. M. (1999). Evaluating the public health impact of health promotion interventions: The RE-AIM framework. Am J Public Health, 89(9), 1322-1327.

Gnambs, T., \& Kaspar, K. (2016). Socially Desirable Responding in Web-Based Questionnaires: A Meta-Analytic Review of the Candor Hypothesis. Assessment, 1-17. doi:10.1177/1073191115624547

Gollwitzer, P. M. (1999). Implementation intentions. Strong effects of simple plans. American Psychologist, 54, 493-503.

Green, L., \& Kreuter, M. (1999). The PRECEDE-PROCEED model. In Health promotion planning: an educational approach. Mountain View (CA): Mayfield Publishing Company.

Griffiths, F., Lindenmeyer, A., Powell, J., Lowe, P., \& Thorogood, M. (2006). Why Are Health Care Interventions Delivered Over the Internet? A Systematic Review of the Published Literature. J Med Internet Res, 8(2), e10. doi:10.2196/jmir.8.2.e10

Gunther, M., \& Alligood, M. R. (2002). A discipline-specific determination of high quality nursing care. J Adv Nurs, 38(4), 353-359.

Hakkaart-van Roijen, L., van der Linden, N., Bouwmans, C., Kanters, T., \& Tan, S. S. (2016). Kostenhandleiding: Methodologie van kostenonderzoek en referentieprijzen voor economische evaluaties in de gezondheidszorg. Retrieved from Zorginstituut Nederland: https://www.zorginstituutnederland.nl/over-ons/publicaties/publicatie/2016/02/29/richtlijn-voor-het-uitvoeren-van-economische-evaluaties-in-de-gezondheidszorg

Halcomb, E. J., Furler, J. S., Hermiz, O. S., Blackberry, I. D., Smith, J. P., Richmond, R. L., \& Zwar, N. A. (2015). Process evaluation of a practice nurse-led smoking cessation trial in Australian general practice: views of general practitioners and practice nurses. Fam Pract. doi:10.1093/fampra/cmv041

Hawkins, R. P., Kreuter, M., Resnicow, K., Fishbein, M., \& Dijkstra, A. (2008). Understanding tailoring in communicating about health. Health Educ Res, 23(3), 454-466. doi:10.1093/her/cyn004

Heijndijk, S. M., \& Willemsen, M. (2015). Dutch Tobacco Control: Moving Towards the Right Track? FCTC shadow report 2014 Alliantie Nederland Rookvrij.

Heiligers, P. J. M., Noordman, J., Korevaar, J. C., Dorsman, S., Hingstman, L., Dulmen, A. M. v., \& Bakker, D. H. d. (2012). Kennisvraag: praktijkondersteuners in de huisartspraktijk (POH's), klaar voor de toekomst? Retrieved from Utrecht: http://www.nivel.nl/sites/default/files/bestanden/Rapport-kennisvraag-pohdef.pdf

Henry, S. G., Penner, L. A., \& Eggly, S. (2017). Associations between thin slice ratings of affect and rapport and perceived patient-centeredness in primary care: Comparison of audio and video recordings. Patient Educ Couns, 100(6), 1128-1135. doi:https://doi.org/10.1016/j.pec.2016.12.020 
Henselmans, I., Heijmans, M., Rademakers, J., \& van Dulmen, S. (2015). Participation of chronic patients in medical consultations: patients' perceived efficacy, barriers and interest in support. Health Expect, 18(6), 23752388. doi:10.1111/hex.12206

Hersch, R. K., Cook, R. F., Deitz, D. K., Kaplan, S., Hughes, D., Friesen, M. A., \& Vezina, M. (2016). Reducing Nurses' Stress: A Randomized Controlled Trial of a Web-Based Stress Management Program for Nurses. Appl Nurs Res, 32, 18-25. doi:10.1016/j.apnr.2016.04.003

Houts, P. S., Doak, C. C., Doak, L. G., \& Loscalzo, M. J. (2006). The role of pictures in improving health communication: a review of research on attention, comprehension, recall, and adherence. Patient Educ Couns, 61(2), 173-190. doi:10.1016/j.pec.2005.05.004

Hoving, C., Mudde, A. N., \& de Vries, H. (2006). Intention to adopt a smoking cessation expert system within a self-selected sample of Dutch general practitioners. Eur J Cancer Prev, 15(1), 82-86.

Hoving, C., Mudde, A. N., \& de Vries, H. (2007). Intention to implement a smoking cessation intervention in Dutch general practice. Health Education, 107(3), 307-315. doi:doi:10.1108/09654280710742591

Hoving, C., Mudde, A. N., Dijk, F., \& de Vries, H. (2010). Effectiveness of a computer-generated tailored smoking cessation expert system in Dutch community pharmacy and general practice setting. Health Education, 110(1), 17-29.

Hummel, K., Willemsen, M. C., de Vries, H., Monshouwer, K., \& Nagelhout, G. E. (2017). Social Acceptance of Smoking Restrictions During 10 Years of Policy Implementation, Reversal, and Reenactment in the Netherlands: Findings From a National Population Survey. Nicotine \& Tobacco Research, 19(2), 231-238. doi:10.1093/ntr/ntw169

Husereau, D., Drummond, M., Petrou, S., Carswell, C., Moher, D., Greenberg, D., ... Loder, E. (2013). Consolidated Health Economic Evaluation Reporting Standards (CHEERS)--explanation and elaboration: a report of the ISPOR Health Economic Evaluation Publication Guidelines Good Reporting Practices Task Force. Value Health, 16(2), 231-250. doi:10.1016/j.jval.2013.02.002

Hutchesson, M. J., Rollo, M. E., Krukowski, R., Ells, L., Harvey, J., Morgan, P. J., . . . Collins, C. E. (2015). eHealth interventions for the prevention and treatment of overweight and obesity in adults: a systematic review with meta-analysis. Obes Rev, 16(5), 376-392. doi:10.1111/obr.12268

Huygens, M. (2018). A patient perspective on eHealth in primary care: Critical reflections on the implementation and use of online care services. Maastricht University, Maastricht, the Netherlands.

Internet World Stats. (2017). Internet Users in the World. Retrieved from http://www.internetworldstats.com/ stats.htm

Jager, C., Steinhauser, J., Freund, T., Baker, R., Agarwal, S., Godycki-Cwirko, M., . . Wensing, M. (2016). Process evaluation of five tailored programs to improve the implementation of evidence-based recommendations for chronic conditions in primary care. Implement Sci, 11(1), 123. doi:10.1186/s13012-016-0473-8

Janssen, M. F., Pickard, A. S., Golicki, D., Gudex, C., Niewada, M., Scalone, L., . . Busschbach, J. (2013). Measurement properties of the EQ-5D-5L compared to the EQ-5D-3L across eight patient groups: a multi-country study. Qual Life Res, 22(7), 1717-1727. doi:10.1007/s11136-012-0322-4

Janz, N. K., Champion, V. L., \& Strecher, V. J. (2002). The Health Belief Model. In K. Glanz (Ed.), Health Behavior and Health Education: Theory, Research, and Practice. San Francisco: Jossey-Bass.

Jaspers, M. W. (2009). A comparison of usability methods for testing interactive health technologies: methodological aspects and empirical evidence. Int J Med Inform, 78(5), 340-353. doi:10.1016/j.ijmedinf.2008.10.002

Jochems, E. C., Mulder, C. L., Duivenvoorden, H. J., van der Feltz-Cornelis, C. M., \& van Dam, A. (2014). Measures of motivation for psychiatric treatment based on self-determination theory: psychometric properties in dutch psychiatric outpatients. Assessment, 21(4), 494-510. doi:10.1177/1073191113517928

Johnson, N. W., Lowe, J. C., \& Warnakulasuriya, K. A. (2006). Tobacco cessation activities of UK dentists in primary care: signs of improvement. Br Dent J, 200(2), 85-89. doi:10.1038/sj.bdj.4813148

Joseph, V., West, R. M., Shickle, D., Keen, J., \& Clamp, S. (2011). Key challenges in the development and implementation of telehealth projects. J Telemed Telecare, 17. doi:10.1258/jtt.2010.100315

Karaman, S. (2011). Nurses' perceptions of online continuing education. BMC Med Educ, 11, 86. doi:10.1186/1472-6920-11-86 
Katz, D. A., Paez, M. W., Reisinger, H. S., Gillette, M. T., Weg, M. W., Titler, M. G., . . Ono, S. S. (2014). Implementation of smoking cessation guidelines in the emergency department: a qualitative study of staff perceptions. Addict Sci Clin Pract, 9, 1. doi:10.1186/1940-0640-9-1

Ketelaar, S. M., Nieuwenhuijsen, K., Gärtner, F. R., Bolier, L., Smeets, O., \& Sluiter, J. K. (2014). Mental Vitality @ Work: The effectiveness of a mental module for workers' health surveillance for nurses and allied health professionals, comparing two approaches in a cluster-randomised controlled trial. International Archives of Occupational and Environmental Health, 87(5), 527-538. doi:10.1007/s00420-013-0893-6

Kleinjan, M., Bommelé, J., Verdurmen, J., \& van Laar, M. (2016). Het bespreken van (stoppen met) roken door de huisarts en andere zorgverleners (tandartsen, medisch specialisten en verloskundigen). Retrieved from Utrecht:

Kohl, L. F., Crutzen, R., \& de Vries, N. K. (2013). Online prevention aimed at lifestyle behaviors: a systematic review of reviews. J Med Internet Res, 15(7), e146. doi:10.2196/jmir.2665

Kok, G., Gottlieb, N. H., Peters, G.-J. Y., Mullen, P. D., Parcel, G. S., Ruiter, R. A. C., . . Bartholomew, L. K. (2016). A taxonomy of behaviour change methods: an Intervention Mapping approach. Health Psychol Rev, 10(3), 297-312. doi:10.1080/17437199.2015.1077155

Kok, G., Schaalma, H., Ruiter, R. A., van Empelen, P., \& Brug, J. (2004). Intervention mapping: protocol for applying health psychology theory to prevention programmes. J Health Psychol, 9(1), 85-98. doi:10.1177/1359105304038379

Konijnendijk, A. A. J., Boere-Boonekamp, M. M., Fleuren, M. A. H., Haasnoot, M. E., \& Need, A. (2016). What factors increase Dutch child health care professionals' adherence to a national guideline on preventing child abuse and neglect? Child Abuse \& Neglect, 53, 118-127. doi:https://doi.org/10.1016/ j.chiabu.2015.11.006

Kotb, A., Cameron, C., Hsieh, S., \& Wells, G. (2015). Comparative effectiveness of different forms of telemedicine for individuals with heart failure (HF): a systematic review and network meta-analysis. PLoS One, 10(2), e0118681. doi:10.1371/journal.pone.0118681

Krebs, P., Prochaska, J. O., \& Rossi, J. S. (2010). A meta-analysis of computer-tailored interventions for health behavior change. Prev Med, 51(3-4), 214-221. doi:10.1016/j.ypmed.2010.06.004

Kreuter, M. W., Strecher, V. J., \& Glassman, B. (1999). One size does not fit all: the case for tailoring print materials. Ann Behav Med, 21(4), 276-283.

Kreuter, M. W., \& Wray, R. J. (2003). Tailored and targeted health communication: strategies for enhancing information relevance. Am J Health Behav, 27 Supp/ 3, S227-232.

Krippendorff, K. (2004). Reliability in Content Analysis. Human Communication Research, 30(3), 411-433. doi:10.1111/j.1468-2958.2004.tb00738.x

Krug, S. (2006). Don't make me think. A common sense approach to web usability. Berkeley, CA: New Riders.

Lau, R., Stevenson, F., Ong, B. N., Dziedzic, K., Treweek, S., Eldridge, S., . . Murray, E. (2016). Achieving change in primary care--causes of the evidence to practice gap: systematic reviews of reviews. Implement Sci, 11, 40. doi:10.1186/s13012-016-0396-4

Leitlein, L., Smit, E. S., de Vries, H., \& Hoving, C. (2012). Factors influencing Dutch practice nurses' intention to adopt a new smoking cessation intervention. J Adv Nurs, 68(10), 2185-2194. doi:10.1111/j.13652648.2011.05903.x

Levesque, C. S., Williams, G. C., Elliot, D., Pickering, M. A., Bodenhamer, B., \& Finley, P. J. (2007). Validating the theoretical structure of the Treatment Self-Regulation Questionnaire (TSRQ) across three different health behaviors. Health Educ Res, 22(5), 691-702. doi:10.1093/her/cyl148

Lindson-Hawley, N., Thompson, T. P., \& Begh, R. (2015). Motivational interviewing for smoking cessation. Cochrane Database Syst Rev(3), Cd006936. doi:10.1002/14651858.CD006936.pub3

Lombard, M., Snyder-Duch, J., \& Bracken, C. C. (2002). Content Analysis in Mass Communication: Assessment and Reporting of Intercoder Reliability. Human Communication Research, 28(4), 587-604. doi:10.1111/j.1468-2958.2002.tb00826.x

Lorencatto, F., West, R., Christopherson, C., \& Michie, S. (2013). Assessing fidelity of delivery of smoking cessation behavioural support in practice. Implement Sci, 8, 40. doi:10.1186/1748-5908-8-40 
Luca, N. R., \& Suggs, L. S. (2013). Theory and Model Use in Social Marketing Health Interventions. J Health Commun, 18(1), 20-40. doi:10.1080/10810730.2012.688243

Lugtenberg, M., Burgers, J. S., Besters, C. F., Han, D., \& Westert, G. P. (2011). Perceived barriers to guideline adherence: a survey among general practitioners. BMC Fam Pract, 12, 98. doi:10.1186/1471-2296-12-98

Lustria, M. L., Cortese, J., Noar, S. M., \& Glueckauf, R. L. (2009). Computer-tailored health interventions delivered over the Web: review and analysis of key components. Patient Educ Couns, 74(2), 156-173. doi:10.1016/j.pec.2008.08.023

Lustria, M. L., Noar, S. M., Cortese, J., Van Stee, S. K., Glueckauf, R. L., \& Lee, J. (2013). A meta-analysis of webdelivered tailored health behavior change interventions. J Health Commun, 18(9), 1039-1069. doi:10.1080/10810730.2013.768727

Macdonald, L. M. (2016). Expertise in Everyday Nurse-Patient Conversations: The Importance of Small Talk. Global qualitative nursing research, 3, 2333393616643201. doi:10.1177/2333393616643201

Marlatt, G. A., \& Donovan, D., M. (2009). Relapse Prevention-Maintenance Strategies in the Treatment of Addictive Behaviour (2nd Edition). New York: Guildford Press.

Martin Cantera, C., Puigdomenech, E., Ballve, J. L., Arias, O. L., Clemente, L., Casas, R., . . Granollers, S. (2015). Effectiveness of multicomponent interventions in primary healthcare settings to promote continuous smoking cessation in adults: a systematic review. BMJ Open, 5(10), e008807. doi:10.1136/bmjopen-2015008807

Matzat, U., Snijders, C., \& van der Horst, W. (2009). Effects of different types of progress indicators on drop-out rates in web surveys. Social Psychology, 40(1), 43-52. doi:10.1027/1864-9335.40.1.43

May, C., Finch, T., Mair, F., Ballini, L., Dowrick, C., Eccles, M., . . Heaven, B. (2007). Understanding the implementation of complex interventions in health care: the normalization process model. BMC Health Serv Res, 7, 148. doi:10.1186/1472-6963-7-148

McEwen, A., West, R., \& Preston, A. (2006). Triggering anti-smoking advice by GPs: mode of action of an intervention stimulating smoking cessation advice by GPs. Patient Educ Couns, 62(1), 89-94. doi:10.1016/j.pec.2005.06.008

Meijer, E., Verbiest, M. E. A., Chavannes, N. H., Kaptein, A. A., Assendelft, W. J. J., Scharloo, M., \& Crone, M. R. (2018). Smokers' identity and quit advice in general practice: General practitioners need to focus more on female smokers. Patient Educ Couns, 101(4), 730-737. doi:https://doi.org/10.1016/j.pec.2017.11.009

Melnyk, B. M., Gallagher-Ford, L., Long, L. E., \& Fineout-Overholt, E. (2014). The establishment of evidencebased practice competencies for practicing registered nurses and advanced practice nurses in real-world clinical settings: proficiencies to improve healthcare quality, reliability, patient outcomes, and costs. Worldviews Evid Based Nurs, 11(1), 5-15. doi:10.1111/wvn.12021

Michie, S., Yardley, L., West, R., Patrick, K., \& Greaves, F. (2017). Developing and Evaluating Digital Interventions to Promote Behavior Change in Health and Health Care: Recommendations Resulting From an International Workshop. J Med Internet Res, 19(6), e232. doi:10.2196/jmir.7126

Miller, M., \& Kearney, N. (2004). Guidelines for clinical practice: development, dissemination and implementation. Int J Nurs Stud, 41(7), 813-821. doi:http://dx.doi.org/10.1016/j.ijnurstu.2003.09.005

Miller WR, \& Rollnick SR. (2002). Motivational Interviewing: preparing people for change (2nd ed.). New York, USA: The Guilford Press.

Moloczij, N., Krishnasamy, M., Butow, P., Hack, T. F., Stafford, L., Jefford, M., \& Schofield, P. (2017). Barriers and facilitators to the implementation of audio-recordings and question prompt lists in cancer care consultations: A qualitative study. Patient Educ Couns, 100(6), 1083-1091. doi:10.1016/j.pec.2017.01.005

Moran-Ellis, J., Alexander, V. D., Cronin, A., Dickinson, M., Fielding, J., Sleney, J., \& Thomas, H. (2006). Triangulation and integration: processes, claims and implications. Qualitative Research, 6(1), 45-59. doi:10.1177/1468794106058870

Morgenroth, T., Ryan, M. K., \& Peters, K. (2015). The motivational theory of role modeling: How role models influence role aspirants' goals. Review of General Psychology, 19(4), 465-483. doi:10.1037/gpr0000059

Mudde, A. N., Willemsen, M. C., Kremers, S., \& de Vries, H. (2006). Meetinstrumenten voor onderzoek naar roken en stoppen met roken. Dutch Expert Center for Tobacco Control, The Hague. 
Murphy, K. R., \& Davidshofer, C. O. (2005). Psychological Testing: Principles and Applications (6th ed.). Upper Saddle River, N.J.: Pearson/Prentice Hall.

Naughton, F., Hopewell, S., Lathia, N., Schalbroeck, R., Brown, C., Mascolo, C., . . Sutton, S. (2016). A ContextSensing Mobile Phone App (Q Sense) for Smoking Cessation: A Mixed-Methods Study. JMIR Mhealth Uhealth, 4(3), e106. doi:10.2196/mhealth.5787

Nederlands Huisartsen Genootschap \& Landelijke Huisarsten Vereniging. (2011). NHG/LHV Standpunt: Het (ondersteunend) Team in de huisartsenvoorziening. Retrieved from Utrecht: https://www.nhg.org/sites/ default/files/content/nhg_org/uploads/toekomstvisie_-_nhg-lhv-standpunt._het_ondersteunend_team_ in_de_huisartsenvoorziening_juni_2011_.pdf

Nguyen, M. H., Smets, E. M. A., Bol, N., Loos, E. F., \& Van Weert, J. C. M. (2018). How Tailoring the Mode of Information Presentation Influences Younger and Older Adults' Satisfaction with Health Websites. J Health Commun, 23(2), 170-180. doi:10.1080/10810730.2017.1421729

Nicastro, E., Lo Vecchio, A., Liguoro, I., Chmielewska, A., De Bruyn, C., Dolinsek, J., . . Guarino, A. (2015). The Impact of E-Learning on Adherence to Guidelines for Acute Gastroenteritis: A Single-Arm Intervention Study. PLoS One, 10(7), e0132213. doi:10.1371/journal.pone.0132213

NICE. (2018). Stop smoking interventions and services. Retrieved from London, UK: nice.org.uk/guidance/ng92 Nielsen, J., \& Mack, R. L. (1994). Usability Inspection Methods. New York: John Wiley \& Sons.

Noordman, J., Koopmans, B., Korevaar, J. C., van der Weijden, T., \& van Dulmen, S. (2013). Exploring lifestyle counselling in routine primary care consultations: the professionals' role. Fam Pract, 30(3), 332-340. doi:10.1093/fampra/cms077

Noordman, J., van der Weijden, T., \& van Dulmen, S. (2014). Effects of video-feedback on the communication, clinical competence and motivational interviewing skills of practice nurses: a pre-test posttest control group study. J Adv Nurs. doi:10.1111/jan.12376

Noordman, J., van Lee, I., Nielen, M., Vlek, H., van Weijden, T., \& van Dulmen, S. (2012). Do trained practice nurses apply motivational interviewing techniques in primary care consultations? J Clin Med Res, 4(6), 393401. doi:10.4021/jocmr1120w

Noordman, J., Verheij, R., \& Verhaak, P. (2008). De inzet van doktersassistenten en praktijkondersteuners in de huisartspraktijk. Retrieved from Utrecht:

Osterwalder, A., \& Pigneur, Y. (2013). Designing business models and similar strategic objects: the contribution of IS. Journal of the Association for Information Systems, 14(5), 237-244.

Page, S. J., \& Persch, A. C. (2013). Recruitment, Retention, and Blinding in Clinical Trials. The American Journal of Occupational Therapy, 67(2), 154-161. doi:10.5014/ajot.2013.006197

Papadakis, S., Tulloch, H. E., Gharib, M., \& Pipe, A. L. (2016). Profile of tobacco users identified in primary care practice and predictors of readiness to quit: a cross-sectional survey. CMAJ Open, 4(1), E41-47. doi:10.9778/cmajo.20150055

Patnode, C. D., Henderson, J. T., Thompson, J. H., Senger, C. A., Fortmann, S. P., \& Whitlock, E. P. (2015). U.S. Preventive Services Task Force Evidence Syntheses, formerly Systematic Evidence Reviews. In Behavioral Counseling and Pharmacotherapy Interventions for Tobacco Cessation in Adults, Including Pregnant Women: A Review of Reviews for the U.S. Preventive Services Task Force. Rockville (MD): Agency for Healthcare Research and Quality (US).

Patrick, K., Hekler, E. B., Estrin, D., Mohr, D. C., Riper, H., Crane, D., . . Riley, W. T. (2016). The Pace of Technologic Change: Implications for Digital Health Behavior Intervention Research. Am J Prev Med, 51(5), 816824. doi:https://doi.org/10.1016/j.amepre.2016.05.001

Pieterse, M. E., Seydel, E. R., de Vries, H., Mudde, A. N., \& Kok, G. J. (2001). Effectiveness of a Minimal Contact Smoking Cessation Program for Dutch General Practitioners: A Randomized Controlled Trial. Prev Med, 32(2), 182-190. doi:https://doi.org/10.1006/pmed.2000.0791

Pinget, C., Martin, E., Wasserfallen, J. B., Humair, J. P., \& Cornuz, J. (2007). Cost-effectiveness analysis of a European primary-care physician training in smoking cessation counseling. Eur J Cardiovasc Prev Rehabil, 14(3), 451-455. doi:10.1097/HJR.0b013e32804955a0

Prochaska, J. O., \& Velicer, W. F. (1997). The transtheoretical model of health behavior change. Am J Health Promot, 12(1), 38-48. 
Raad voor de Volksgezondheid en Zorg. (2006). Zinnige en duurzame zorg: transparante keuzen in de zorg voor een houdbaar zorgstelsel. Raad voor de Volksgezondheid en zorg: Zoetermeer.

Reif, S., Horgan, C. M., \& Ritter, G. A. (2011). Treatment services: triangulation of methods when there is no gold standard. Subst Use Misuse, 46(5), 620-632. doi:10.3109/10826084.2010.528119

Rice, V. H., Hartmann-Boyce, J., \& Stead, L. F. (2013). Nursing interventions for smoking cessation. Cochrane Database Syst Rev, 8, CD001188. doi:10.1002/14651858.CD001188.pub4

Rice, V. H., Heath, L., Livingstone-Banks, J., \& Hartmann-Boyce, J. (2017). Nursing interventions for smoking cessation. Cochrane Database Syst Rev, 12, Cd001188. doi:10.1002/14651858.CD001188.pub5

RIVM. (2014). Volksgezondheid Toekomst Verkenning (VTV) 'Een gezonder Nederland'. Retrieved from Bilthoven:http://www.rivm.nl/dsresource?objectid=rivmp:251654\&type=org\&disposition=inline\&ns_nc=1,

Rogers, E. M. (2003). Diffusion of Innovations (5th ed.). New York: Free Press.

Rutten, G. M., Degen, S., Hendriks, E. J., Braspenning, J. C., Harting, J., \& Oostendorp, R. A. (2010). Adherence to clinical practice guidelines for low back pain in physical therapy: do patients benefit? Phys Ther, 90(8), 1111-1122. doi:10.2522/ptj.20090173

Salmon, P., Peters, S., Rogers, A., Gask, L., Clifford, R., Iredale, W., . . Morriss, R. (2007). Peering through the barriers in GPs' explanations for declining to participate in research: the role of professional autonomy and the economy of time. Fam Pract, 24(3), 269-275. doi:10.1093/fampra/cmm015

Sangster-Gormley, E., Martin-Misener, R., Downe-Wamboldt, B., \& Dicenso, A. (2011). Factors affecting nurse practitioner role implementation in Canadian practice settings: an integrative review. J Adv Nurs, 67(6), 1178-1190. doi:10.1111/j.1365-2648.2010.05571.x

Sarna, L., Bialous, S. A., Wells, M., \& Brook, J. (2017). Impact of a webcast on nurses' delivery of tobacco dependence treatment. J Clin Nurs. doi:10.1111/jocn.13875

Saunders, H., Vehviläinen-Julkunen, K., \& Stevens, K. R. (2016). Effectiveness of an education intervention to strengthen nurses' readiness for evidence-based practice: A single-blind randomized controlled study. Applied Nursing Research, 31, 175-185. doi:http://dx.doi.org/10.1016/j.apnr.2016.03.004

Schafer, J. L., \& Graham, J. W. (2002). Missing data: our view of the state of the art. Psychol Methods, 7(2), 147-177.

Schneider, F., van Osch, L., \& de Vries, H. (2012). Identifying factors for optimal development of health-related websites: a delphi study among experts and potential future users. J Med Internet Res, 14(1), e18. doi:10.2196/jmir.1863

Segaar, D., Bolman, C., Willemsen, M., \& De Vries, H. (2007). Identifying determinants of protocol adoption by midwives: a comprehensive approach. Health Educ Res, 22(1), 14-26. doi:10.1093/her/cyl046

Segaar, D., Willemsen, M. C., Bolman, C., \& De Vries, H. (2007). Nurse adherence to a minimal-contact smoking cessation intervention on cardiac wards. Res Nurs Health, 30(4), 429-444. doi:10.1002/nur.20204

Selby, P., Goncharenko, K., Barker, M., Fahim, M., Timothy, V., Dragonetti, R., . . Hays, J. T. (2015). Review and Evaluation of Online Tobacco Dependence Treatment Training Programs for Health Care Practitioners. J Med Internet Res, 17(4), e97. doi:10.2196/jmir.3284

Shaw, E. K., Howard, J., West, D. R., Crabtree, B. F., Nease, D. E., Jr., Tutt, B., \& Nutting, P. A. (2012). The role of the champion in primary care change efforts: from the State Networks of Colorado Ambulatory Practices and Partners (SNOCAP). J Am Board Fam Med, 25(5), 676-685. doi:10.3122/jabfm.2012.05.110281

Shiffman, S., Patten, C., Gwaltney, C., Paty, J., Gnys, M., Kassel, J., . . Balabanis, M. (2006). Natural history of nicotine withdrawal. Addiction, 101(12), 1822-1832. doi:10.1111/j.1360-0443.2006.01635.x

Sinclair, P. M., Kable, A., Levett-Jones, T., \& Booth, D. (2016). The effectiveness of Internet-based e-learning on clinician behaviour and patient outcomes: A systematic review. Int J Nurs Stud, 57, $70-81$. doi:http://dx.doi.org/10.1016/j.ijnurstu.2016.01.011

Smeets, O., \& Zijlstra-Vlasveld, M. (2016). Implementatie 'Blended e-(mental) health in de huisartsenpraktijk': Resultaten en lessons learned van de implementatie van blended Kleurjeleven in de huisartsenzorg. Retrieved from Utrecht:

Smit, E. S., Candel, M. J., Hoving, C., \& de Vries, H. (2016). Results of the PAS study: A randomized controlled trial evaluating the effectiveness of a web-based multiple tailored smoking cessation program combined with tailored counseling by practice nurses. Health Commun, 1-9. doi:10.1080/10410236.2015.1049727 
Smit, E. S., de Vries, H., \& Hoving, C. (2010). The PAS study: a randomized controlled trial evaluating the effectiveness of a web-based multiple tailored smoking cessation programme and tailored counselling by practice nurses. Contemp Clin Trials, 31(3), 251-258. doi:10.1016/j.cct.2010.03.001

Smit, E. S., de Vries, H., \& Hoving, C. (2012). Effectiveness of a Web-based multiple tailored smoking cessation program: a randomized controlled trial among Dutch adult smokers. J Med Internet Res, 14(3), e82. doi:10.2196/jmir.1812

Smit, E. S., de Vries, H., \& Hoving, C. (2013). Determinants of practice nurses' intention to implement a new smoking cessation intervention: the importance of attitude and innovation characteristics. J Adv Nurs, 69(12), 2665-2674. doi:10.1111/jan.12153

Smit, E. S., de Vries, H., Oberjé, E. J. M., \& Evers, S. M. A. A. (2015). Easier said than done: overcoming challenges in the economic evaluation of internet-based lifestyle interventions. The European Health Psychologist, 17(1), 39-44.

Smit, E. S., Evers, S. M., de Vries, H., \& Hoving, C. (2013). Cost-effectiveness and cost-utility of Internet-based computer tailoring for smoking cessation. J Med Internet Res, 15(3), e57. doi:10.2196/jmir.2059

Smit, E. S., Hoving, C., Schelleman-Offermans, K., West, R., \& de Vries, H. (2014). Predictors of successful and unsuccessful quit attempts among smokers motivated to quit. Addict Behav(0). doi:http://dx.doi.org/10.1016/j.addbeh.2014.04.017

Smit, E. S., Linn, A. J., \& van Weert, J. C. M. (2015). Taking online computer-tailoring forward: The potential of tailoring the message frame and delivery mode of online health behaviour change interventions. The European Health Psychologist, 17(1), 25-31.

Smith, P. M., Sellick, S. M., \& Spadoni, M. M. (2012). Tobacco cessation Clinical Practice Guideline use by rural and urban hospital nurses: a pre-implementation needs assessment. BMC Nurs, 11, 6. doi:10.1186/14726955-11-6

Soetens, K. C., Vandelanotte, C., de Vries, H., \& Mummery, K. W. (2014). Using online computer tailoring to promote physical activity: a randomized trial of text, video, and combined intervention delivery modes. J Health Commun, 19(12), 1377-1392. doi:10.1080/10810730.2014.894597

Spelten, E. R., Martin, L., Gitsels, J. T., Pereboom, M. T., Hutton, E. K., \& van Dulmen, S. (2015). Introducing video recording in primary care midwifery for research purposes: procedure, dataset, and use. Midwifery, 31(1), 95-102. doi:10.1016/j.midw.2014.06.007

Springvloet, L., \& van Laar, M. (2017). Roken onder volwassenen: kerncijfers 2016. Retrieved from Utrecht:

Stanczyk, N., Bolman, C., van Adrichem, M., Candel, M., Muris, J., \& de Vries, H. (2014). Comparison of text and video computer-tailored interventions for smoking cessation: randomized controlled trial. J Med Internet Res, 16(3), e69. doi:10.2196/jmir.3016

Stanczyk, N. E., Bolman, C., Muris, J. W., \& de Vries, H. (2011). Study protocol of a Dutch smoking cessation ehealth program. BMC Public Health, 11, 847. doi:10.1186/1471-2458-11-847

Stanczyk, N. E., Smit, E. S., Schulz, D. N., de Vries, H., Bolman, C., Muris, J. W., \& Evers, S. M. (2014). An economic evaluation of a video- and text-based computer-tailored intervention for smoking cessation: a cost-effectiveness and cost-utility analysis of a randomized controlled trial. PLoS One, 9(10), e110117. doi:10.1371/journal.pone.0110117

Stead, L. F., Buitrago, D., Preciado, N., Sanchez, G., Hartmann-Boyce, J., \& Lancaster, T. (2013). Physician advice for smoking cessation. Cochrane Database Syst Rev, 5, CD000165. doi:10.1002/14651858. CD000165.pub4

Stead, L. F., Koilpillai, P., Fanshawe, T. R., \& Lancaster, T. (2016). Combined pharmacotherapy and behavioural interventions for smoking cessation. Cochrane Database Syst Rev, 3, Cd008286. doi:10.1002/14651858. CD008286.pub3

Stead, L. F., Koilpillai, P., \& Lancaster, T. (2015). Additional behavioural support as an adjunct to pharmacotherapy for smoking cessation. Cochrane Database Syst Rev, 10, Cd009670. doi:10.1002/14651858. CD009670.pub3

Stop Smoking Partnership. (2009). Guideline treatment tobacco addiction [Richtlijn behandeling van tabaksvers/aving]. Retrieved from Alphen aan den Rijn, The Netherlands: http://www.partnershipstopmetroken.nl/ richtlijn-2/, 
Studts, J. L., Flynn, S. M., Dill, T. C., Ridner, S. L., Worth, C. T., Walsh, S. E., \& Sorrell, C. L. (2010). Nurse Practitioners' Knowledge, Attitudes, and Clinical Practices Regarding Treatment of Tobacco Use and Dependence. The Journal for Nurse Practitioners, 6(3), 212-219. doi:http://dx.doi.org/10.1016/j.nurpra.2009.06.003

Swan, M., Ferguson, S., Chang, A., Larson, E., \& Smaldone, A. (2015). Quality of primary care by advanced practice nurses: a systematic review. Int J Qual Health Care, 27(5), 396-404. doi:10.1093/intqhc/mzv054

Swinkels, I. C. S., Huygens, M. W. J., Schoenmakers, T. M., Oude Nijeweme-D'Hollosy, W., van Velsen, L., Vermeulen, J., . . . de Witte, L. (2018). Lessons Learned From a Living Lab on the Broad Adoption of eHealth in Primary Health Care. J Med Internet Res, 20(3), e83. doi:10.2196/jmir.9110

Talboom-Kamp, E. P. W. A., Verdijk, N. A., Kasteleyn, M. J., Numans, M. E., \& Chavannes, N. H. (2018). From chronic disease management to person-centered eHealth; a review on the necessity for blended care. Clinical eHealth, 1(1), 3-7. doi:https://doi.org/10.1016/j.ceh.2018.01.001

Tate, D. F., Finkelstein, E. A., Khavjou, O., \& Gustafson, A. (2009). Cost Effectiveness of Internet Interventions: Review and Recommendations. Annals of Behavioral Medicine, 38(1), 40-45. doi:10.1007/s12160-009-9131-6

Taylor, G. M. J., Dalili, M. N., Semwal, M., Civljak, M., Sheikh, A., \& Car, J. (2017). Internet-based interventions for smoking cessation. Cochrane Database Syst Rev, 9, Cd007078. doi:10.1002/14651858.CD00 7078.pub5

Te Poel, F., Bolman, C., Reubsaet, A., \& de Vries, H. (2009). Efficacy of a single computer-tailored e-mail for smoking cessation: results after 6 months. Health Educ Res.

Toepoel, V., Das, M., \& Van Soest, A. (2009). Design of Web Questionnaires: The Effects of the Number of Items per Screen. Field Methods, 21(2), 200-213. doi:10.1177/1525822x08330261

Trimbos-instituut, \& NHG. (2016). Richtlijn Behandeling van tabaksverslaving en stoppen met roken ondersteuning Herziening 2016. Retrieved from

Unrod, M., Simmons, V. N., Sutton, S. K., Cummings, K. M., Celestino, P., Craig, B. M., . . Brandon, T. H. (2016). Relapse-Prevention Booklets as an Adjunct to a Tobacco Quitline: A Randomized Controlled Effectiveness Trial. Nicotine Tob Res, 18(3), 298-305. doi:10.1093/ntr/ntv079

Unrod, M., Smith, M., Spring, B., DePue, J., Redd, W., \& Winkel, G. (2007). Randomized controlled trial of a computer-based, tailored intervention to increase smoking cessation counseling by primary care physicians. J Gen Intern Med, 22(4), 478-484. doi:10.1007/s11606-006-0069-0

USDHHS. (2014). The Health Consequences of Smoking - 50 Years of Progress. A Report of the Surgeon General. Retrieved from Atlanta, GA: http://www.surgeongeneral.gov/library/reports/50-years-of-progress/ fullreport.pdf

Valente, T. W., \& Pumpuang, P. (2007). Identifying opinion leaders to promote behavior change. Health Educ Behav, 34(6), 881-896. doi:10.1177/1090198106297855

van Bruinessen, I. R., Leegwater, B., \& van Dulmen, S. (2017). When patients take the initiative to audio-record a clinical consultation. Patient Educ Couns, 100(8), 1552-1557. doi:http://dx.doi.org/10.1016/ j.pec.2017.03.001

van de Glind, I., Heinen, M., Geense, W., Mesters, I., Wensing, M., \& van Achterberg, T. (2015). Making the connection-factors influencing implementation of evidence supported and non-evaluated lifestyle interventions in healthcare: a multiple case study. Health Educ Res, 30(4), 521-541. doi:10.1093/her/cyv020

Van de Mortel, T. F. (2008). Faking it: social desirability response bias in self-report research.

van de Steeg, L., IJkema, R., Wagner, C., \& Langelaan, M. (2015). The effect of an e-learning course on nursing staff's knowledge of delirium: a before-and-after study. BMC Medical Education, 15(1), 12. doi:10.1186/s12909-015-0289-2

Van den Berg, M., \& Schoemaker, C. G. (2010). Effecten van preventie. Deelrapport van de Volksgezondheid Toekomst Verkenning 2010 Van gezond naar beter. RIVM-rapport nr. 270061007, Bilthoven: RIVM.

van der Weide, M., \& Smits, J. (2004). Adoption of innovations by specialised nurses: personal, work and organisational characteristics. Health Policy, 68(1), 81-92. doi:http://dx.doi.org/10.1016/ j.healthpol.2003.09.007

van Gelder, M. M., Bretveld, R. W., \& Roeleveld, N. (2010). Web-based questionnaires: the future in epidemiology? Am J Epidemiol, 172(11), 1292-1298. doi:10.1093/aje/kwq291 
van Gemert-Pijnen, J. E., Nijland, N., van Limburg, M., Ossebaard, H. C., Kelders, S. M., Eysenbach, G., \& Seydel, E. R. (2011). A holistic framework to improve the uptake and impact of eHealth technologies. J Med Internet Res, 13(4), e111. doi:10.2196/jmir.1672

van Hassel, D., Batenburg, R., \& van der Velden, L. (2016). Praktijkondersteuners (POH's) in beeld: Aantallen, kenmerken en geografische spreiding in Nederland. Retrieved from Utrecht:

van Laar, M. W., van Ooyen-Houben, M. M. J., Cruts, A. A. N., Meijer, R. F., Croes, E. A., Ketelaars, A. P. M., \& van der Pol, P. M. (2016). Nationale Drug Monitor. Retrieved from

van Limburg, M., Wentzel, J., Sanderman, R., \& van Gemert-Pijnen, L. (2015). Business Modeling to Implement an eHealth Portal for Infection Control: A Reflection on Co-Creation With Stakeholders. JMIR Res Protoc, 4(3), e104. doi:10.2196/resprot.4519

van Rossem, C., Spigt, M., Viechtbauer, W., Lucas, A. E. M., van Schayck, O. C. P., \& Kotz, D. (2017). Effectiveness of intensive practice nurse counselling versus brief general practitioner advice, both combined with varenicline, for smoking cessation: a randomised pragmatic trial in primary care. Addiction. doi:10.1111/add.13927

van Rossem, C., Spigt, M. G., Kleijsen, J. R., Hendricx, M., van Schayck, C. P., \& Kotz, D. (2015). Smoking cessation in primary care: Exploration of barriers and solutions in current daily practice from the perspective of smokers and healthcare professionals. Eur J Gen Pract, 1-7. doi:10.3109/13814788.2014.990881

Vangeli, E., Stapleton, J., Smit, E. S., Borland, R., \& West, R. (2011). Predictors of attempts to stop smoking and their success in adult general population samples: a systematic review. Addiction, 106(12), 2110-2121. doi:10.1111/j.1360-0443.2011.03565.x

Verbiest, M. E., Chavannes, N. H., Passchier, E., Noordman, J., Scharloo, M., Kaptein, A. A., . . . Crone, M. R. (2014). Sequence-analysis of video-recorded practitioner-patient communication about smoking in general practice: Do smokers express negative statements about quitting? Patient Educ Couns. doi:10.1016/j.pec.2014.08.006

Verbiest, M. E., Crone, M. R., Scharloo, M., Chavannes, N. H., van der Meer, V., Kaptein, A. A., \& Assendelft, W. J. (2014). One-hour training for general practitioners in reducing the implementation gap of smoking cessation care: a cluster-randomized controlled trial. Nicotine Tob Res, 16(1), 1-10. doi:10.1093/ntr/ntt100

Versteegh, M. M., Vermeulen, K. M., Evers, S. M. A. A., de Wit, G. A., Prenger, R., \& Stolk, E. A. (2016). Dutch Tariff for the Five-Level Version of EQ-5D. Value Health, 19(4), 343-352. doi:10.1016/j.jval.2016.01.003

Vervloet, M., Dijk, L., Bakker, D. H., Souverein, P. C., Santen-Reestman, J., Vlijmen, B., . . Bouvy, M. L. (2014). Short- and long-term effects of real-time medication monitoring with short message service (SMS) reminders for missed doses on the refill adherence of people with Type 2 diabetes: evidence from a randomized controlled trial. Diabetic Medicine, 31(7), 821-828. doi:doi:10.1111/dme.12439

Vervloet, M., Linn, A. J., van Weert, J. C. M., de Bakker, D. H., Bouvy, M. L., \& van Dijk, L. (2012). The effectiveness of interventions using electronic reminders to improve adherence to chronic medication: a systematic review of the literature. Journal of the American Medical Informatics Association, 19(5), 696-704. doi:10.1136/amiajnl-2011-000748

Vervloet, M., van Dijk, L., Santen-Reestman, J., van Vlijmen, B., Bouvy, M. L., \& de Bakker, D. H. (2011). Improving medication adherence in diabetes type 2 patients through Real Time Medication Monitoring: a randomised controlled trial to evaluate the effect of monitoring patients' medication use combined with short message service (SMS) reminders. BMC Health Serv Res, 11, 5. doi:10.1186/1472-6963-11-5

Voncken-Brewster, V., Moser, A., van der Weijden, T., Nagykaldi, Z., de Vries, H., \& Tange, H. (2013). Usability evaluation of an online, tailored self-management intervention for chronic obstructive pulmonary disease patients incorporating behavior change techniques. JMIR Res Protoc, 2(1), e3. doi:10.2196/resprot.2246

Voogt, M. P., Opmeer, B. C., Kastelein, A. W., Jaspers, M. W. M., \& Peute, L. W. (2018). Obstacles to Successful Implementation of eHealth Applications into Clinical Practice. Stud Health Technol Inform, 247, 521-525.

Waldorff, F. B., Steenstrup, A. P., Nielsen, B., Rubak, J., \& Bro, F. (2008). Diffusion of an e-learning programme among Danish General Practitioners: A nation-wide prospective survey. BMC Fam Pract, 9(1), 24. doi:10.1186/1471-2296-9-24 
Walters, E. L., Reibling, E. T., Wilber, S. T., Sullivan, A. F., Gaeta, T. J., Camargo, C. A., Jr., \& Boudreaux, E. D. (2014). Emergency Department Provider Preferences Related to Clinical Practice Guidelines for Tobacco Cessation: A Multicenter Survey. Acad Emerg Med, 21(7), 785-793. doi:10.1111/acem.12421

Walthouwer, M. J., Oenema, A., Lechner, L., \& de Vries, H. (2015). Comparing a Video and Text Version of a Web-Based Computer-Tailored Intervention for Obesity Prevention: A Randomized Controlled Trial. J Med Internet Res, 17(10), e236. doi:10.2196/jmir.4083

Walthouwer, M. J. L., Oenema, A., Candel, M., Lechner, L., \& de Vries, H. (2015). Eating in moderation and the essential role of awareness. A Dutch longitudinal study identifying psychosocial predictors. Appetite, 87(0), 152-159. doi:http://dx.doi.org/10.1016/j.appet.2014.12.214

Warner, K. E., \& Burns, D. M. (2003). Hardening and the hard-core smoker: concepts, evidence, and implications. Nicotine \& Tobacco Research, 5(1), 37-48. doi:10.1080/1462220021000060428

Webb, T. L., Sniehotta, F. F., \& Michie, S. (2010). Using theories of behaviour change to inform interventions for addictive behaviours. Addiction, 105(11), 1879-1892. doi:10.1111/j.1360-0443.2010.03028.x

Whitehead, D., Zucker, S. B., \& Stone, J. (2014). Tobacco cessation education for advanced practice nurses. Nurse Educ, 39(5), 252-255. doi:10.1097/nne.0000000000000056

WHO. (2013). WHO report on the global tobacco epidemic 2013: Enforcing bans on tobacco advertising, promotion and sponsorship. Retrieved from Geneva: http://apps.who.int/iris/bitstream/10665/85380/1/ 9789241505871_eng.pdf?ua=1

WHO. (2015). WHO report on the global tobacco epidemic 2015: Raising taxes on tobacco. Retrieved from Geneva: http://apps.who.int/iris/bitstream/10665/178574/1/9789240694606_eng.pdf?ua=1\&ua=1

WHO. (2017). WHO report on the global tobacco epidemic, 2017: monitoring tobacco use and prevention policies. Geneva: World Health Organization.

Williams, G. C., Grow, V. M., Freedman, Z. R., Ryan, R. M., \& Deci, E. L. (1996). Motivational predictors of weight loss and weight-loss maintenance. J Pers Soc Psychol, 70(1), 115-126.

Williams, G. C., McGregor, H. A., Sharp, D., Levesque, C., Kouides, R. W., Ryan, R. M., \& Deci, E. L. (2006). Testing a self-determination theory intervention for motivating tobacco cessation: supporting autonomy and competence in a clinical trial. Health Psychol, 25(1), 91-101. doi:10.1037/0278-6133.25.1.91

Williams, G. C., Niemiec, C. P., Patrick, H., Ryan, R. M., \& Deci, E. L. (2009). The importance of supporting autonomy and perceived competence in facilitating long-term tobacco abstinence. Ann Behav Med, 37(3), 315. doi:10.1007/s12160-009-9090-y

Wollersheim, H., Hermens, R., Hulscher, M., Braspenning, J., Ouwens, M., Schouten, J., .. Grol, R. (2007). Clinical indicators: development and applications. Neth J Med, 65(1), 15-22.

Woolf, S. H., Grol, R., Hutchinson, A., Eccles, M., \& Grimshaw, J. (1999). Clinical guidelines: potential benefits, limitations, and harms of clinical guidelines. BMJ, 318(7182), 527-530.

Yardley, L., Spring, B. J., Riper, H., Morrison, L. G., Crane, D. H., Curtis, K., .. Blandford, A. (2016). Understanding and Promoting Effective Engagement With Digital Behavior Change Interventions. Am J Prev Med, 51(5), 833-842. doi:10.1016/j.amepre.2016.06.015

Zhao, J., Zhai, Y. K., Zhu, W. J., \& Sun, D. X. (2015). Effectiveness of Telemedicine for Controlling Asthma Symptoms: A Systematic Review and Meta-analysis. Telemed J E Health, 21(6), 484-492. doi:10.1089/tmj.2014.0119

Zorginstituut Nederland. (2017a). Farmacotherapeutisch kompas. Retrieved from www.farmacotherapeutischkompas.nl

Zorginstituut Nederland. (2017b). Medicijnkosten.nl. Retrieved from www.medicijnkosten.nl 

Summary 
Smoking prevalence in the Netherlands continues to be above the global average, as almost one in four adults smoke. Most Dutch smokers can be reached through their general practice for smoking cessation support, but application of the national smoking cessation guideline by practice nurses is not optimal. As this limits the effectiveness of practice nurse-led smoking cessation counseling, it is important to explore their counseling in more detail and to develop guideline adherence support for practice nurses. Therefore, the aim of the present dissertation was to provide detailed insight in the current smoking cessation counseling practices of practice nurses in Dutch general practice and the (costleffectiveness of a web-based computer-tailored e-learning program aimed to improve practice nurses' adherence to the national evidence-based smoking cessation guideline.

Chapter 1 provides a general introduction to chapters 2-6 of this dissertation. It presents important background information, and details on the theoretical grounding (i.e. IChange Model) and specific research questions of the studies reported on in this dissertation.

Chapter 2 describes the results from a qualitative exploration of practice nurses' guideline adherence and needs for adherence support. All practice nurses $(N=17)$ reported to use an evidence-based guideline, but that they also experienced difficulties in optimally applying such a guideline in practice. A prominent psychological barrier was practice nurses' low self-efficacy to fully adhere to the guideline, resulting in difficulties to increase motivation of smoking patients and to arrange adequate follow-up consultations after a planned quit date. Frequently mentioned practical barriers were practice nurses' perception of a lack of high-quality smoking cessation training opportunities and perceived difficulties in obtaining up-to-date information on rules and regulations concerning compensation of counseling for smokers. To help overcome these barriers, practice nurses believed that their general practitioner could have a facilitating role by systematically providing all smokers with a quit advice (instead of motivated smokers only) and more often referring smokers to the practice nurse for structured counseling. Additionally, practice nurses expressed interest in a support program that could stimulate and remind them to apply an evidence-based smoking cessation guideline during consultations. Practice nurses were specifically interested in an easy-to-use program that could directly be consulted and applied in practice, against minimal time investment.

Chapter 3 provides insight in the role of socio-cognitive determinants of practice nurses' guideline adherence by describing the results of a web-based questionnaire among practice nurses $(\mathrm{N}=157)$. Both determinants of practice nurses' adherence to the complete guideline (i.e. overall adherence) and individual guideline steps (i.e. step-based adherence) were investigated. Concerning overall adherence, practice nurses on average adhered to five out of nine steps of the evidence-based smoking cessation guideline, which was positively associated with higher levels of self-efficacy to use a guideline and spending more time on counseling. Results on step-based adherence showed that practice 
nurses' optimal adherence to individual guideline steps ranged between 34-75\% (e.g. optimal adherence for 'providing a quit advice' was 57\%). Step-based adherence was fairly consistently, positively associated with higher levels of self-efficacy to use a guideline and spending more time on counseling, and in addition adherence to several steps was positively associated with perceived advantages of using a guideline.

Chapter 4 describes the development of a web-based computer-tailored e-learning program to support Dutch practice nurses to improve their smoking cessation guideline adherence. The e-learning program included three modules with tailored advice, an online forum, modules with up-to-date information about smoking cessation, frequently asked questions and project information, and a counseling checklist. Results from chapters 2 and 3 were incorporated by targeting important barriers to adherence and socio-cognitive determinants associated with adherence in this computer-tailored e-learning program. By also taking practice nurses' needs regarding the design and content of the program into account, a program was created that could be used by practice nurses in a flexible way (i.e. wherever and whenever they wanted), containing counseling information for practice nurses and smokers, as well as access to practical experiences of peers through the online forum. Ultimately, improving alterations concerning the program's usability were incorporated to simplify navigation through the program (e.g. add return-to-home buttons) and optimize the length of program content (e.g. breakdown of tailored advice into small pieces of text), based on evaluations with both practice nurses and experts.

Chapter 5 presents the results of a randomized controlled effectiveness trial concerning the effects of the e-learning program on guideline adherence of practice nurses ( $N=269)$. In this trial, intervention group practice nurses had full access to the e-learning program for six months, and were compared to control group practice nurses with limited program access (i.e. access to frequently asked questions, project information and counseling checklist only). Multilevel effect analyses revealed that more experienced practice nurses in the intervention group had better overall adherence scores than their less experienced colleagues in the control group, which was also found for practice nurses' adherence to several individual counseling steps. Additionally, step-based adherence was found to be related to practice nurses' lower baseline levels of several socio-cognitive factors (e.g. self-efficacy and perceived advantages), meaning that intervention group practice nurses could improve more on these factors and were apparently better able to translate this into adequate guideline adherence.

Chapter 6 provides a description of the economic evaluation that was conducted as part of the effectiveness trial. This study was conducted on both practice nurse level and patient level, to investigate the cost-effectiveness of the e-learning program to improve practice nurses' guideline adherence and patients' smoking abstinence. On practice nurse level, cost-effectiveness analyses illustrated that the e-learning program was likely 
to be cost-effective in improving practice nurses' guideline adherence, as $€ 1,586$ has to be paid for every additional guideline step that is adhered to. On patient level, no effects on smoking abstinence were found, but a significant reduction in intervention group patients' nicotine dependence was found after six months, which disappeared after twelve months. Cost-effectiveness analyses on patient level showed that the e-learning program was moderately likely to be cost-effective after six months (i.e. costs for each additional quitter were $€ 7,126)$, and not cost-effective after twelve months to improve patients' smoking abstinence. Cost-utility analysis on patient level demonstrated moderately likelihood of the program's cost-effectiveness to improve patients' quality of life (costs for each additional Quality Adjusted Life Year gained were $€ 18,431$ ). The results of our economic evaluation hence illustrated that the e-learning program was cost-effective to improve practice nurses' guideline adherence, but not cost-effective to indirectly improve patients' smoking abstinence.

Chapter 7 provides a general discussion of the results presented in chapters 2-6 of this dissertation, leading to recommendations for research and practice. In this chapter it is recommended to 1) invest in more personal contact and detailed analyses of recruitment strategies to improve patients' participation rate in research trials; 2) apply strategies, such as mode-tailoring and sending tailored reminders, to improve practice nurses' program use; 3) develop a measure of guideline adherence, such as clinical vignettes, that is free from limitations concerning patient data collection and recording consultations; 4) conduct more research concerning the patient-provider relationship, practice nurses' motivational interviewing skills and patients' quit motivation; 5) pursue national implementation of the e-learning program by applying systematic, evidence-based implementation strategies. 
Samenvatting 
Bijna een op de vier volwassenen in Nederland rookt. Daarmee ligt de prevalentie van het aantal rokers in Nederland boven het wereldwijde gemiddelde. De meeste Nederlandse rokers kunnen in hun huisartsenpraktijk bereikt worden voor ondersteuning bij het stoppen met roken. Het toepassen van de nationale stoppen-met-roken richtlijn door praktijkondersteuners (POHs) is echter niet optimaal. Dit heeft als gevolg dat de effectiviteit van de stoppen-met-roken begeleiding door POHs beperkt is. Het is daarom belangrijk om hun begeleiding gedetailleerd te onderzoeken en ondersteuning te bieden aan POHs zodat de richtlijn beter nageleefd wordt. Het doel van dit proefschrift was om gedetailleerd inzicht te geven in de huidige stoppen-met-roken-begeleiding door POHs in Nederlandse huisartsenpraktijken. Daarnaast was het onze doelstelling om inzicht te krijgen in de (kosten)effectiviteit van een online advies-op-maat (e-learning) programma dat als doel had het toepassen van de nationale evidence-based stoppen-met-roken richtlijn te verbeteren.

Hoofdstuk 1 geeft een algemene inleiding tot de hoofdstukken 2-6 van dit proefschrift. Er wordt belangrijke achtergrondinformatie gepresenteerd, evenals de details met betrekking tot theoretische onderbouwing (d.w.z. het I-Change Model), en beschrijft de specifieke onderzoeksvragen van de verschillende studies beschreven in dit proefschrift.

Hoofdstuk 2 presenteert de resultaten van een kwalitatief onderzoek naar de naleving van de stoppen-met-roken-richtlijn door $\mathrm{POH}$ en beschrijft de behoeftes met betrekking tot het krijgen van ondersteuning bij het toepassen van de richtlijn. Alle POHs ( $N=17)$ rapporteerde het gebruik van een evidence-based richtlijn, maar tevens dat ze moeilijkheden ervaarden in het optimaal toepassen van zo'n richtlijn in de praktijk. Een belangrijke psychologische barrière van de $\mathrm{POH}$ was een lage eigen-effectiviteit om de richtlijn zo volledig mogelijk na te leven, wat resulteert in moeilijkheden om de motivatie van rokers te verhogen maar ook om passende follow-up afspraken te maken na een geplande stopdatum. Veelgenoemde praktische barrières waren een waargenomen gebrek aan kwalitatief goede stoppen-met-roken trainingsmogelijkheden en moeilijkheden met betrekking tot het verkrijgen van actuele informatie over de compensatie van de begeleiding van rokers. Om barrières te overkomen zou de huisarts volgens de POHs een faciliterende rol kunnen hebben bij het systematisch aanbieden van een stoppen-met-roken advies (in plaats van deze alleen te geven aan gemotiveerde rokers) en zouden rokers vaker naar een $\mathrm{POH}$ doorgestuurd kunnen worden voor structurele begeleiding. Tevens gaven $\mathrm{POHs}$ aan geïnteresseerd te zijn in een programma waarin ze gestimuleerd werden en herinneringen ontvingen om de evidence-based stoppen-met-roken richtlijn toe te passen tijdens afspraken. POHs waren specifiek geïnteresseerd in een makkelijk toegankelijk programma dat direct geraadpleegd en toegepast kon worden met een minimale tijdsinvestering.

Hoofdstuk 3 geeft inzicht in de rol van socio-cognitieve determinanten in het toepassen van de richtlijn door POHs door het beschrijven van de resultaten van een online vragenlijst onder POHs ( $\mathrm{N}=157)$. Zowel determinanten van het volledig toepassen van de richtlijn 
door POHs (d.w.z. algemene naleving) als van het uitvoeren van individuele richtlijn stappen (d.w.z. stapsgewijze naleving) zijn onderzocht. Gekeken naar het volledig naleven van de richtlijn in het algemeen dan bleek dat de $\mathrm{POHs}$ gemiddeld vijf van de negen stappen toepasten, en dit was positief geassocieerd met een hogere mate van eigen-effectiviteit om de richtlijn toe te passen en met meer tijd die besteed werd aan de begeleiding. Resultaten van de stapsgewijze naleving lieten zien dat de optimale toepassing van de individuele stappen door POHs varieerde tussen de 43-75\% (bijvoorbeeld: de optimale toepassing van 'het geven van een stopadvies' was 57\%). Stapsgewijze naleving was redelijk consistent geassocieerd met wederom een hogere mate van eigen-effectiviteit om de richtlijn toe te passen en met meer bestede tijd aan de begeleiding. Daarnaast was het toepassen van de stappen positief geassocieerd met waargenomen voordelen van het gebruiken van een richtlijn.

Hoofdstuk 4 illustreert de ontwikkeling van een e-learning programma om Nederlandse $\mathrm{POH}$ ondersteuning te bieden bij het verbeteren van het toepassen van de stoppen-metroken richtlijn. Het e-learning programma bevatte drie modules met advies-op-maat, een online forum, modules met recente informatie over stoppen-met-roken, veel gestelde vragen en projectinformatie, en een counseling checklist. De resultaten uit hoofdstuk 2 en 3 waren opgenomen in het e-learning programma door de inhoud te richten op belangrijke barrières voor naleving van de richtlijn en socio-cognitieve determinanten geassocieerd met richtlijn naleving. Daarnaast zijn ook de wensen en behoefte van de POHs met betrekking tot de vormgeving en de inhoud van het programma in acht genomen zodat het mogelijk was een programma te creëren dat door de $\mathrm{POHs}$ op een flexibele manier (d.w.z. waar en wanneer ze dat wilden) gebruikt kon worden. Het programma bevatte informatie voor zowel POHs als rokers en gaf ook toegang tot praktische ervaringen van collega-POHs via het online forum. Als laatste werd de gebruiksvriendelijkheid van het programma getest door POHs en experts. Op basis hiervan werden verbeteringen doorgevoerd met betrekking tot het navigeren door het programma (bijvoorbeeld het toevoegen van return-to-home knoppen) en het optimaliseren van de lengte van de inhoud van het programma (bijvoorbeeld het opdelen van advies-op-maat in kleine stukjes tekst).

Hoofdstuk 5 presenteert de resultaten van een gerandomiseerd onderzoek met controlegroep naar de effecten van het e-learning programma op het toepassen van de richtlijn door POHs ( $\mathrm{N}=269)$. In deze studie hadden $\mathrm{POH}$ in de interventiegroep zes maanden lang volledige toegang tot het e-learning programma. De interventiegroep werd vervolgens vergeleken met de controlegroep bestaande uit POHs met beperkte toegang tot het programma (d.w.z. alleen toegang tot de veel gestelde vragen, projectinformatie en de counseling checklist). Multilevel analyses lieten zien dat meer ervaren POHs in de interventiegroep de richtlijn in het algemeen beter naleefden dan hun minder ervaren collega's uit de controlegroep. Hetzelfde werd gevonden voor naleving van verschillende individuele 
richtlijn stappen. Daarnaast werd gevonden dat de stapsgewijze naleving van POHs gerelateerd was aan het baseline niveau van verscheidene socio-cognitieve factoren, zoals eigen-effectiviteit en waargenomen voordelen. Dit betekent dat POHs in de interventiegroep zich meer op deze factoren konden ontwikkelen en daarnaast ook beter in staat waren dit te vertalen naar een adequate toepassing van de richtlijn.

Hoofdstuk 6 beschrijft de economische evaluatie, die onderdeel was van de effectiviteitsstudie. Deze studie was uitgevoerd op zowel POH-niveau als patiënt-niveau, zodat de kosteneffectiviteit van het e-learning programma onderzocht konden worden om zowel richtlijn naleving door $\mathrm{POH}$ te verbeteren en stoppen-met-roken onder hun patiënten te verbeteren. Op POH-niveau lieten de analyses zien dat het e-learning programma zeer waarschijnlijk kosteneffectief was om de toepassing van de richtlijn te verbeteren. Voor elke extra stap die door een $\mathrm{POH}$ wordt nageleefd moet $€ 1,586$ worden betaald ten opzichte van de controlegroep. Op patiënt-niveau werden er geen effecten gevonden gekeken naar het stoppen-met-roken, al bleek de nicotine-afhankelijkheid onder patiënten in de interventiegroep na zes maanden significant verminderd te zijn. Na twaalf maanden was dit effect echter verdwenen. Kosteneffectiviteitsanalyses op patiënt-niveau lieten zien dat het e-learning programma mogelijk kosteneffectief was na zes maanden (d.w.z. de kosten voor iedere additionele patiënt die stopt met roken was €7,126), maar niet kosteneffectief na twaalf maanden. Kostenutiliteitsanalyses op patiënt-niveau demonstreerde een matige kans dat het programma kosteneffectief was met betrekking tot het verbeteren van de kwaliteit van het leven van de patiënten (kosten voor ieder additioneel levensjaar in goede gezondheid waren $€ 18,431$ ). De resultaten van onze economische evaluatie laten zien dat het e-learning programma kosteneffectief is als het gaat om het verbeteren van richtlijn naleving onder POHs, maar niet kosteneffectief om indirect het stoppen-met-roken bij patiënten te verbeteren.

Hoofdstuk 7 geeft een algemene discussie van de resultaten gepresenteerd in hoofdstukken 2-6 van dit proefschrift, welke leiden tot aanbevelingen voor onderzoek en praktijk. In dit hoofdstuk wordt er aanbevolen om 1) te investeren in meer persoonlijk contact en gedetailleerde analyses van wervingsstrategieën om het aantal deelnemende patiënten in studies te verbeteren; 2) strategieën zoals mode-tailoring en het versturen van opmaat-gemaakte herinneringen toe te passen om programma gebruik door $\mathrm{POHs}$ te verbeteren; 3) een uitkomstmaat voor richtlijn toepassing te ontwikkelen, zoals klinische vignetten, vrij van beperkingen als het gaat om het verzamelen van patiënten data en het opnemen van gesprekken; 4) meer onderzoek uit te voeren naar de patiënt-provider relatie, de motiverende gespreksvaardigheden van $\mathrm{POH}$ en de motivatie van patiënten om te stoppen-met-roken; 5) nationale implementatie van het e-learning programma na te streven door middel van het toepassing van systematische, evidence-based implementatie strategieën. 
Dankwoord 
Uiteraard wil ik iedereen bedanken die de afgelopen jaren op welke manier dan ook heeft bijgedragen aan het tot stand komen van mijn proefschrift.

Als eerste natuurlijk de deelnemers aan de verschillende studies die in mijn proefschrift zijn beschreven. Mijn waardering gaat uit naar de vele praktijkondersteuners (POHs) die de afgelopen jaren een centrale plek hadden binnen mijn promotieonderzoek. Als onderdeel van de meer praktische kanten van het project (zoals werving, interviews afnemen, contacten onderhouden) heb ik regelmatig het enthousiasme mogen ervaren van de deelnemende POHs. Hun deelname was essentieel in zowel de ontwikkeling als ook de evaluatie van het e-learning programma, en zonder de inzet van de $\mathrm{POH}$ hadden we geen evaluatie kunnen doen onder rokende patiënten.

Een speciaal woord van dank gaat uit naar mijn promotieteam. Ciska, Eline en Hein, jullie hebben mij deze kans gegeven en ik waardeer het gevoel van vertrouwen dat ik vanaf het begin van het project heb ervaren.

Ciska, bedankt voor je tijd en je geduld tijdens onze overleggen. Op wekelijkse basis investeerde jij in mijn ontwikkeling tot onderzoeker: je stimuleerde en steunde mij om allerlei congressen en cursussen te bezoeken; je leerde me om het meeste uit onze overleggen te halen; en je wist me telkens weer richting de juiste balans tussen detail-hoofdzaak te leiden, zonder hierbij wetenschappelijke integriteit uit het oog te verliezen. Ik kon altijd bij je binnen lopen voor een korte vraag of om ergens over te sparren; heel fijn die openheid! Dat ik de afgelopen jaren veel van je heb geleerd, blijkt wel uit een opmerking die ik laatst kreeg: mijn manier van feedback geven kwam een collega namelijk bekend voor...!

Eline, toen ik met mijn project startte stond jij op het punt met je baan in Amsterdam te beginnen. Ondanks dat we dus maar weinig face-to-face samengewerkt hebben, heb ik altijd het gevoel gehad dat je zeer aanwezig en betrokken was bij mijn project. Buiten onze reguliere overleggen, benadrukte je regelmatig dat ik je altijd kon bellen en mailen als dat nodig was, en heb dit als erg prettig ervaren. Daarnaast heb ik veel geleerd van je inhoudelijke feedback en praktische ervaringen uit je eigen project met $\mathrm{POH}$ en rokers.

Hein, of moet ik zeggen 'advocaat van de duivel', want zo noemde je jezelf regelmatig tijdens onze maandelijkse overleggen. Jij zorgde vaak voor een extra kritische blik op het project, vanuit helikopter perspectief. Op basis van jouw jarenlange ervaring en expertise vroeg jij je vaak af wat je als reviewer of referent van een gekozen aanpak zou vinden, zodat ik vervolgens beter voorbereid was op mogelijke valkuilen (zorg voor een 'plan B') en gestimuleerd werd om de gekozen aanpak nog verder uit te werken. Bedankt ook voor de nauwere betrokkenheid bij het project wanneer dit noodzakelijk was; het was immers lastig om van tevoren in mijn planning rekening te houden met het zwangerschapsverlof van twee-derde van het team. 
Jullie unieke combinatie van invloeden op mijn promotieproject werkte heel fijn, en ik weet zeker dat dit mij heeft geholpen om de kwaliteit van mijn proefschrift naar een hoger niveau te tillen!

Zoals al even benoemd bestond een aanzienlijk deel van mijn project uit praktische zaken. Gelukkig heb ik de afgelopen jaren hierbij hulp gehad van een fantastisch team, zonder wie het niet mogelijk zou zijn geweest het project tot een goed einde te brengen. Claire, Linda en Raesita, heel erg bedankt voor de prettige samenwerking en jullie enthousiaste inzet voor het project. Jullie zijn onmisbaar geweest in het ondersteunen van allerlei activiteiten en taken, zoals het uitvoeren en uittypen van interviews, het ontwikkelen en testen van het e-learning programma, het maken en verspreiden van nieuwsbrieven, en nog zo veel meer! Ook de vele uren aan de telefoon voor het werven van voldoende deelnemers mag hierbij niet vergeten worden; hiervoor waren ook Anke en Anuska essentieel om te voorkomen dat we niet al te veel achter raakte op schema. Bedankt!

Ook wil ik verschillende organisaties bedanken voor hun medewerking tijdens het project. Mede dankzij de steun van organisaties zoals Verpleegkundigen \& Verzorgenden Nederland, de Nederlandse Vereniging van Praktijkondersteuners, de Alliantie Nederland Rookvrij en het Kwaliteitsregister stoppen met roken is het gelukt om ons e-learning programma onder een breed publiek onder de aandacht te brengen. In het bijzonder wil ik Leonie Keizer, Dewi Segaar, Toos Jacobs, Helene Reulen en Paul van Spiegel bedanken; jullie waren minstens zo enthousiast als ik over het project en stonden altijd klaar om mee te denken en mee te helpen met betrekking tot het bereiken van $\mathrm{POH}$ in heel $\mathrm{Ne}$ derland.

Daarnaast wil ik mijn dankbaarheid uitspreken richting de leden van de beoordelingscommissie van mijn proefschrift. Stef, Catherine, Niels, Jean en Marc, bedankt voor jullie tijd om mijn proefschrift te lezen en te beoordelen. Trudy, Marcia, Lisette en Matty, bedankt dat jullie bereid zijn om onderdeel te zijn van de corona tijdens mijn verdediging.

Co-auteurs, bedankt voor de prettige samenwerking tijdens het schrijven van de artikelen die onderdeel zijn van mijn proefschrift. Math en Silvia, ik heb erg veel geleerd van jullie waardevolle inzichten op het gebied van multilevel effect analyses en kosteneffectiviteit analyses.

Toppers van de vakgroep, oftewel Patricia, Leon en Kim, bedankt voor jullie ondersteuning de afgelopen jaren. Jullie stonden en staan nog steeds altijd voor me klaar en houden de vakgroep draaiende. Ik kan me echt geen vakgroep zonder jullie voorstellen, merci!

Uiteraard ook een woord van dank voor alle andere (ex-)collega's. Jullie hebben ervoor gezorgd dat de vakgroep veel meer is dan alleen een werkplek, maar dat er zeker ook ruimte is voor gezelligheid en afleiding. Ik heb genoten van de verscheidenheid aan (PhD-)lunches, 
TMO-uitjes en de vele spontane of minder spontane VrijMiBo's! Francine, laten we die borrels er inhouden. Belangrijker nog, ik hoop dat we nog lang bij elkaar terecht kunnen voor steun en afleiding; je bent een topper, en ik ben heel blij dat je mijn paranimf wilt zijn!

Ik wil ook mijn kamergenoten bedanken. Karin, jij hielp mij vanaf het begin direct fijn op weg. Via jou kwam ik er pas echt achter wat er allemaal van je verwacht wordt als promovendus, en vooral ook hoe ik hier mijn eigen draai aan kon geven zodat ik het tot een goed einde wist te brengen. Ik probeer die mate van steun nu zelf als kamergenoot door te geven aan Sacha. Een oude bekende, en wat mij betreft óók een ideale kamergenoot omdat we dezelfde balans hebben tussen hard werken en gezelligheid.

Mannen, bedaank! Wat hebben wij toch een super vriendengroep, echt heel waardevol. Voor de uitlaatklep en afleiding wanneer nodig, of dit nou een mannenweekend in de Ardennen is of speciaal biertjes drinken bij de Gouverneur. Maar daarnaast ook voor de steun en een luisterend oor op momenten dat daar vraag naar was. Ik hoop van harte dat onze bijzondere vriendschap nog lang mag duren.

Lieve pap, mam en Nadine, ontzettend bedankt voor jullie onvoorwaardelijk steun. Jullie stonden altijd voor me klaar, ook wanneer het op het werk of daar buiten wat minder ging. Dit heeft mij de kracht gegeven om altijd de draad weer op te pakken, positief te blijven en te geloven in een goede afloop. En Nadine, ik vind het bijzonder dat je me bij wilt staan bij de verdediging als mijn paranimf.

En tenslotte wil ik jou bedanken liefste Niki. Je onvoorwaardelijk vertrouwen in mij en jouw vermogen om dingen te relativeren hielpen mij om het ook tijdens de laatste loodjes vol te houden. Bedankt ook voor het overnemen van taken in huis, wanneer ik nog aan het werk was, en voor het meedenken in het ontwerpen van mijn proefschrift. Het is zo ontzettend fijn dat je er altijd voor me bent! Ik kijk ernaar uit om samen met jou nog heel veel mooie dingen te mogen meemaken, en om samen een toekomst te bouwen. 
Curriculum Vitae 
Dennis Franciscus Ludovicus de Ruijter was born on February 15th 1990 in Maastricht, The Netherlands. After graduating from secondary school (Gymnasium, VWO Porta Mosana Maastricht) in 2008 he started the Bachelor Life Sciences at University College Maastricht. As a part of his bachelor program, Dennis completed a semester abroad at the Australian National University in Canberra, Australia. After graduation in 2011, he started a master's program in Sports and Physical Activity Interventions at Maastricht University. One year later he graduated.

In 2012-2013 Dennis worked as a human movement scientist at the local professional soccer club MVV Maastricht, to apply his knowledge about the human body to further improve the players' performance. During this period, he experienced that conducting research was an interesting challenge that he would like to pursue in a more systematic and controlled way. In the meantime, he collaborated with a health coaching professional to gain additional research experience. Being involved with a professional that devoted her work to help other people improve their lifestyle and experiencing the positive effects of health counselling, Dennis became more and more interested in working in the field of health promotion.

In January 2014, Dennis started working as a PhD candidate at the department of Health Promotion of Maastricht University, under supervision of dr. Ciska Hoving, dr. Eline Smit and prof. dr. Hein de Vries. During four and half years he worked on several studies that are described in this dissertation, which he combined with a variety of teaching responsibilities at Maastricht University. Currently, Dennis continues to be involved in both research and teaching as he is appointed as postdoctoral researcher at the department of Health Promotion. 


\section{Publication list}


de Ruijter, D., Smit, E. S., de Vries, H., Goossens, L., \& Hoving, C. (2017). Understanding Dutch practice nurses' adherence to evidence-based smoking cessation guidelines and their needs for web-based adherence support: results from semistructured interviews. BMJ Open, 7(3), e014154. doi:10.1136/bmjopen-2016-014154

de Ruijter, D., Smit, E. S., de Vries, H., \& Hoving, C. (2017). Dutch practice nurses' adherence to evidence-based smoking cessation treatment guidelines. Fam Pract. doi:10.1093/fampra/cmx039

de Ruijter, D., Smit, E. S., de Vries, H., \& Hoving, C. (2016). Web-based computer-tailoring for practice nurses aimed to improve smoking cessation guideline adherence: A study protocol for a randomized controlled effectiveness trial. Contemp Clin Trials, 48, 125-132. doi:10.1016/j.cct.2016.04.007

de Ruijter, D., Candel, M., Smit, E. S., de Vries, H., \& Hoving, C. (2018). The Effectiveness of a Computer-Tailored E-Learning Program for Practice Nurses to Improve Their Adherence to Smoking Cessation Counseling Guidelines: Randomized Controlled Trial. J Med Internet Res, 20(5), e193. doi:10.2196/jmir.9276

de Ruijter, D., Hoving, C., Evers, S., Hudales, R., de Vries, H., \& Smit, E.S. (2018). An economic evaluation of a computer-tailored e-learning program to promote smoking cessation counseling guideline adherence among practice nurses. (submitted)

\section{Other publications:}

de Ruijter, D., Smit, E.S., de Vries, H., \& Hoving, C. (2015). STER studie Stoppen met roken. Op één Lijn(51), p 21.

de Ruijter, D., Smit, E. S., de Vries, H., \& Hoving, C. (2015). Doe mee met de STER studie. Op één Lijn(53), p. 16.

de Ruijter, D., Smit, E. S., de Vries, H., \& Hoving, C. (2017). Online support programma voor professionals. Inspiratie vakblad voor longverpleegkundigen, 27(1), p. 20.

de Ruijter, D., Smit, E. S., de Vries, H., \& Hoving, C. (2017). Praktijkverpleegkundigen volgen stoppen-met-roken-richtlijn maar gedeeltelijk. Ned Tijdschr Geneeskd, 161(C3502).

Cheung, K. L., de Ruijter, D., Hiligsmann, M., Elfeddali, I., Hoving, C., Evers, S., \& de Vries, H. (2017). Exploring consensus on how to measure smoking cessation. A Delphi study. BMC Public Health, 17(1), 890. doi:10.1186/s12889-017-4902-7 